\title{
TARGETING HUMAN INSULIN SENSITIVITY
}

Citation for published version (APA):

Remie, C. (2020). TARGETING HUMAN INSULIN SENSITIVITY: a cool, an active, and a compoundbased approach. [Doctoral Thesis, Maastricht University]. Ridderprint. https://doi.org/10.26481/dis.20202808cr

Document status and date:

Published: 01/01/2020

DOI:

$10.26481 /$ dis.20202808cr

Document Version:

Publisher's PDF, also known as Version of record

\section{Please check the document version of this publication:}

- A submitted manuscript is the version of the article upon submission and before peer-review. There can be important differences between the submitted version and the official published version of record.

People interested in the research are advised to contact the author for the final version of the publication, or visit the DOI to the publisher's website.

- The final author version and the galley proof are versions of the publication after peer review.

- The final published version features the final layout of the paper including the volume, issue and page numbers.

Link to publication

\footnotetext{
General rights rights.

- You may freely distribute the URL identifying the publication in the public portal. please follow below link for the End User Agreement:

www.umlib.nl/taverne-license

Take down policy

If you believe that this document breaches copyright please contact us at:

repository@maastrichtuniversity.nl

providing details and we will investigate your claim.
}

Copyright and moral rights for the publications made accessible in the public portal are retained by the authors and/or other copyright owners and it is a condition of accessing publications that users recognise and abide by the legal requirements associated with these

- Users may download and print one copy of any publication from the public portal for the purpose of private study or research.

- You may not further distribute the material or use it for any profit-making activity or commercial gain

If the publication is distributed under the terms of Article $25 \mathrm{fa}$ of the Dutch Copyright Act, indicated by the "Taverne" license above, 


\section{TARGETING HUMAN INSULIN SENSITIVITY \\ a cool, an active, and a compound-based approach}

Carlijn Remie 


\section{NUTRIM}

The research presented in this thesis was performed within NUTRIM School of Nutrition and Translational Research in Metabolism. The research described in this thesis was supported by a grant of the Dutch Heart Foundation (CVON2014-02). Financial support by the Dutch Heart Foundation for the publication of this thesis is gratefully acknowledged.

Cover design: Evelien Jagtman - evelienjagtman.com

Layout: Carlijn Remie

Printed by: Ridderprint - ridderprint.nl

ISBN: 978-94-6375-934-2

Copyright @ Carlijn Remie, Maastricht 2020 


\section{TARGETING HUMAN INSULIN SENSITIVITY}

a cool, an active, and a compound-based approach

\section{PROEFSCHRIFT}

ter verkrijging van de graad van doctor aan de Universiteit Maastricht, op gezag van de Rector Magnificus, Prof.dr. Rianne M. Letschert volgens het besluit van het College van Decanen,

in het openbaar te verdedigen

op vrijdag 28 augustus 2020 om 14.00 uur

door

Carolina Maria Elisa Remie

Geboren op 12 maart 1991 te Sittard 


\section{Promotoren}

Prof. Dr. P. Schrauwen

Prof. Dr. M.K.C. Hesselink

Copromotor

Dr. E. Phielix

\section{Beoordelingscommissie}

Prof. Dr. R.P. Mensink (voorzitter)

Dr. D.P. Blondin (Université de Sherbrooke, Québec, Canada)

Prof. Dr. M.C.G.J. Brouwers

Prof. Dr. L.J.C. van Loon

Dr. H.P. van der Ploeg (Amsterdam UMC) 


\section{TABLE OF CONTENTS}

$\begin{array}{lll}\text { CHAPTER } 1 & \text { General introduction and outline } & 7\end{array}$

CHAPTER $2 \quad$ Nicotinamide riboside supplementation alters 23

body composition and skeletal muscle

acetylcarnitine concentrations in healthy obese

humans

CHAPTER 3 Increasing NAD+ levels through nicotinamide

53

riboside activates in vivo brown adipose tissue in

mice but not in humans

CHAPTER $4 \quad$ Non-shivering cold acclimation does not improve postprandial glucose and lipid metabolism or insulin sensitivity in patients with type 2 diabetes

CHAPTER 5 Replacing sedentary time with standing and walking is similar effective in enhancing insulin sensitivity as moderate-to-vigorous exercise

CHAPTER 6 General discussion and conclusion

APPENDICES

Valorization

Summary 153

Samenvatting 157

Dankwoord 161

List of publications 165

About the author 



\section{CHAPTER 1}

General introduction and outline

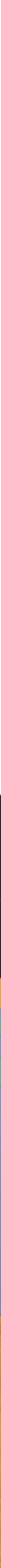





\section{THE OBESITY PROBLEM}

The worldwide prevalence of overweight and obesity have dramatically increased in the past decades (1). This development is also reflected in the Dutch population. In $1990,35 \%$ of the adult Dutch population was overweight, of which $6 \%$ was obese. In 2018 , these numbers increased to $50 \%$ being overweight, of which $15 \%$ was obese (2). Overweight and obesity are recognized as major contributing factors to the development of type 2 diabetes mellitus (T2DM), cardiovascular diseases and nonalcoholic fatty liver disease (3). In 2016, T2DM and cardiovascular diseases were part of the top 5 worldwide causes of death in the age group above 50 years (4). Despite these alarming numbers, the prevalence of overweight and obesity is expected to continue to rise even further in the coming years (5). Given the fact that obesity was once considered a high-income country problem and nowadays these trends appear in low- and middle-income countries as well (1), it has become a worldwide problem. The fundamental cause of obesity is a change in lifestyle leading to a positive energy balance with energy intake exceeding energy expenditure for a prolonged period of time, with body mass gain as a consequence. The underlying explanation for this positive energy balance is in the majority of cases a combination of a high-caloric diet and low levels of physical activity.

\section{INSULIN RESISTANCE AND TYPE 2 DIABETES}

In 2018, it was estimated that around 500 million adults worldwide and almost 1.2 million adults in the Netherlands were diagnosed with diabetes $(6,7)$. Of these numbers, around 90\% can be accounted to type 2 diabetes mellitus (T2DM). T2DM is part of the top 3 diseases with the highest disease burden in the Netherlands (8). The prevalence of T2DM in the Dutch population has been estimated to increase by $34 \%$ in the period 2015-2040 (9). Likewise, the prevalence worldwide will increase as well, with the greatest expected increase in low-income countries (6), rendering T2DM a serious worldwide health problem.

T2DM is characterized by insulin resistance in peripheral tissues, most notably skeletal muscle, liver, and adipose tissue (see Figure 1). As a result, pancreatic insulin secretion will rise in an attempt to compensate for the insulin resistance. During the initial stage of this disease, hyperinsulinemia serves to maintain normoglycemia. Eventually, in a later disease stage, insulin production in the pancreas declines and plasma glucose levels begin to rise. Long term insulin resistance causes chronically increased plasma glucose levels (hyperglycemia), originating from compromised insulin mediated suppression of hepatic glucose output as well as from blunted insulin-stimulated glucose uptake in skeletal muscle (10). Skeletal muscle accounts 
for the major part ( 80\%) of postprandial insulin-mediated glucose disposal (11). Glucose uptake in skeletal muscle occurs via glucose transporter type 4 (GLUT4) translocation from intracellular stores to the cell membrane. Once glucose has entered the cell it can be directed towards oxidation in the mitochondria or be stored as glycogen. Insulin activates the enzyme glycogen synthase thereby promoting postprandial glycogen storage (10). However, during exercise, GLUT4 translocation occurs independently from insulin (12). The insulin mediated GLUT4 translocation and subsequent glycogen synthesis is compromised in patients with type 2 diabetes (10), contributing to hyperglycemia. Patients with T2DM, however, are not resistant to the stimulatory effects of exercise on GLUT4 translocation (13). Normally, in the liver, insulin suppresses hepatic glucose output. Hepatic glucose production originates from glycogenolysis as well as from gluconeogenesis. In case of insulin resistance, the hepatic glucose production is less suppressed in the postprandial state contributing to hyperglycemia (10). Furthermore, insulin resistance in white adipose tissue leads to a decrease in insulin mediated lipid storage and a decreased suppression of lipolysis, with a consequent rise of plasma fatty acid levels (10). This spillover of fatty acids from the white adipose tissue leads to lipid storage in nonadipose tissues like skeletal muscle and liver, also referred to as ectopic lipid storage (14). This ectopic lipid storage deleteriously affects cell function and again has been associated with insulin resistance and type 2 diabetes (14). Thus, insulin resistance in overweight and obese people forms a major risk for the development of T2DM. Eventually, long term hyperglycemia in T2DM patients, can lead to complications, including cardiovascular diseases, nephropathy, neuropathy and retinopathy (15).

\section{THE ROLE OF MITOCHONDRIA}

Mitochondria are cellular organelles which are essential for aerobic energy production. Inside the mitochondria, substrates are converted into ATP with the consumption of oxygen via oxidative phosphorylation. ATP provides chemical energy to fuel vital cellular processes, for example muscle contractions. To adapt to oscillations in energy demand and substrate availability, healthy mitochondria can switch readily between oxidative metabolism of fatty acids and glucose, a process often referred to as metabolic flexibility. In the metabolically inflexible state, the switch from predominantly fatty acids in the fasted state to more glucose oxidation in the postprandial state is blunted. This results in delayed postprandial glucose clearance and to disturbances in glucose homeostasis (16), such as hyperglycemia. 


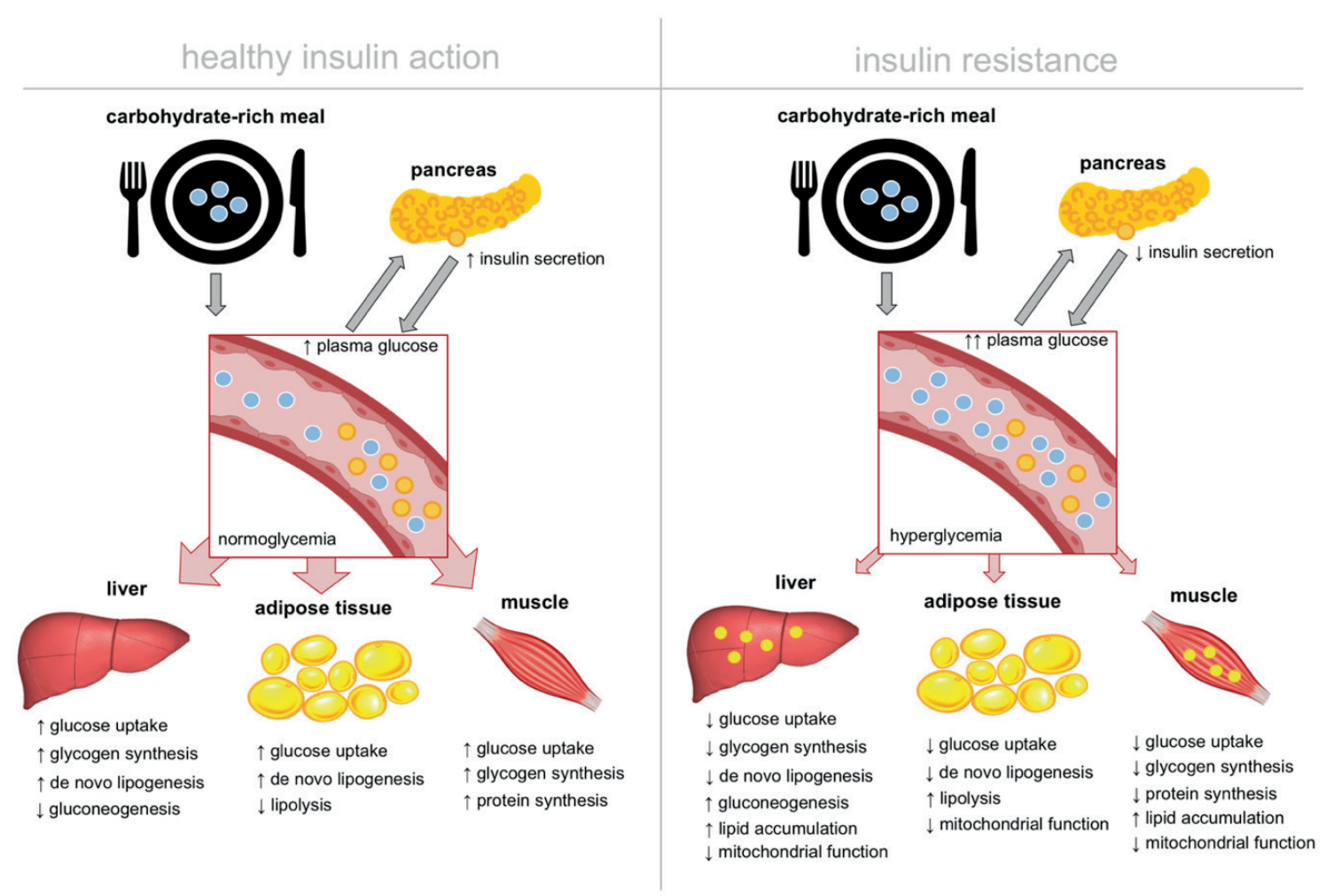

Figure 1. Mechanisms of healthy insulin action and insulin resistance

Furthermore, a compromised mitochondrial function can result in impaired fat oxidation and thereby may play a role in ectopic lipid accumulation (17). It has been reported that mitochondrial number and function decline with normal aging $(18,19)$ and a compromised mitochondrial function has been associated with insulin resistance, type 2 diabetes and cardiovascular diseases $(20,21)$.

\section{WELL KNOWN INTERVENTION STRATEGIES TO IMPROVE INSULIN SENSITIVITY}

In many cases, obesity can be prevented or treated through lifestyle interventions. Exercise and caloric restriction are the two most commonly studied and powerful lifestyle interventions for counteracting obesity and metabolic disturbances including T2DM $(22,23)$. Both strategies are suggested to act partly via activation of AMPactivated protein kinase (AMPK) and sirtuin 1 (SIRT1). Both AMPK and SIRT1 are activated during cellular energy crisis, by changes in energy charge. Downstream activation of AMPK and SIRT1 results in many metabolic adaptations, including enhanced mitochondrial biogenesis and function, mostly through activation of peroxisome proliferator-activated receptor gamma co-activator 1-alpha (PGC-1a) (24-26). 


\section{Caloric restriction}

Creating a negative energy balance by reducing energy intake (caloric restriction) results in weight loss and is the most effective intervention strategy to increase lifespan in a wide range of species, including humans (27). Regarding the metabolic benefits, several intervention studies in overweight humans investigated six months of caloric restriction by $25 \%$ reduction in energy intake and reported reduced fasting insulin levels (28), increased gene expression of SIRT1 and PGC-1a $(28,29)$, decreased hepatic lipid accumulation (30), increased insulin sensitivity (30) and enhanced mitochondrial biogenesis (29). These studies were accompanied by $10 \%$ weight loss. Weight loss has been extensively recognized to improve insulin sensitivity and mitochondrial function by creating a negative energy balance and reducing ectopic lipid storage $(31,32)$.

\section{Exercise}

Exercise training is one of the most potent ways to promote peripheral glucose disposal and to increase muscle mitochondrial function. Numerous human intervention studies have examined the effect of a wide range of exercise training programs on markers of insulin sensitivity and mitochondrial function in humans with obesity (33-36), patients with $\operatorname{T2DM}(33,34)$ and aged people (37), some studies combined with weight loss (32). Exercise exerts significant improvements in glucose metabolism and insulin sensitivity. During an exercise session, the increased skeletal muscle energy demand and concomitant AMPK activation promotes GLUT4 translocation and stimulates substrate oxidation (38). In addition, several postexercise adaptations occur and share the common purpose to replenish fuel stores, particularly muscle and liver glycogen. To illustrate, increased membrane bound GLUT4 in skeletal muscle and improved insulin sensitivity of skeletal muscle and liver have been observed in lean healthy individuals up to 48 hours after the last exercise session $(38,39)$.

\section{INVESTIGATION OF NOVEL STRATEGIES TO IMPROVE INSULIN SENSITIVITY}

Although these strategies seem very effective, long term compliance to dietary changes or adherence to exercise programs are usually very low in the general population $(40,41)$. Therefore, novel strategies to improve human metabolic health, affecting insulin sensitivity and mitochondrial function, are urgently needed. This has led to a search for novel intervention strategies, which can initiate the beneficial metabolic health effects similar to those from caloric restriction and exercise training. Within this thesis three novel approaches will be investigated (see Figure 2). These approaches will be briefly introduced below. 


\section{Promoting NAD+ metabolism}

Nicotinamide adenosine dinucleotide (NAD) plays a central role in energy metabolism. NAD occurs in the oxidized form as $\mathrm{NAD}^{+}$or in the reduced form as NADH. NAD serves as an electron carrier in the oxidation of substrates. High ratios of $\mathrm{NAD}^{+} / \mathrm{NADH}$ reflect a low cellular energy charge and induces activation of sirtuins. Sirtuins are $\mathrm{NAD}^{+}$-dependent deacetylating enzymes that function as metabolic regulators (42). Seven members of the sirtuin family (SIRT1-7) have been identified in humans, of which SIRT1 is the most widely studied family member. SIRT1 is activated in situations of energy stress, such as caloric restriction and exercise (43) and NAD ${ }^{+}$ is the rate limiting substrate for SIRT1 (44). Thus, SIRT1 is a potentially powerful molecular target for the prevention and treatment of obesity and T2DM.

Recently, $\mathrm{NAD}^{+}$increasing compounds have been examined with respect to their SIRT1 activating potential. The increase in $\mathrm{NAD}^{+}$can be achieved by inhibition of $\mathrm{NAD}^{+}$degradation pathways as well as by activating NAD+ ${ }^{+}$synthesis pathways. NAD+ can be synthesized de novo from the essential amino-acid tryptophan, which is present in our diet. However, the main source of $\mathrm{NAD}^{+}$derives from salvage pathways, which requires uptake of $\mathrm{NAD}^{+}$precursors from our diet. Several NAD+ precursors have been identified, including nicotinic acid (NA), Acipimox, nicotinamide (NAM), nicotinamide mononucleotide (NMN) and nicotinamide riboside (NR). The use of NA and the NA-analog Acipimox dates back to the 1980's and was used to treat hyperlipidemia (45). However, more recent studies have shown that Acipimox is also capable of increasing cellular NAD+ levels and enhanced mitochondrial function in humans are reported (46), suggesting that supplementation with NAD+ precursors is a promising strategy to improve human metabolic health. Most of these nicotinamide related compounds, however, have been linked to unwanted side effects (flushing) and are therefore not preferable targets for the treatment of chronic metabolic diseases $(47,48)$. NMN and NR can be considered a new generation of $\mathrm{NAD}^{+}$ precursors that do not provoke these side effects. In animal models, NMN and NR supplementation have been reported to improve oxidative metabolism, mitochondrial function and insulin sensitivity (49-53).

Since 2016, NR supplements have been available for human use and have been shown to increase circulatory NAD+ levels (54). In 2017, the pharmacokinetics and human safety of NR supplementation were described $(55,56)$. This opens new avenues for human clinical trials to examine if NR supplementation can improve insulin sensitivity, mitochondrial function and other metabolic health related parameters. 


\section{Cold acclimation and brown adipose tissue}

Besides targeting $\mathrm{NAD}^{+}$metabolism, another approach to improve human metabolic health is cold exposure. Physiological responses to cold exposure serve to maintain core body temperature stable at $\sim 37^{\circ} \mathrm{C}$ include: 1 ) insulative responses by peripheral vasoconstriction and 2) metabolic responses by increasing energy expenditure. A fundamental component of human physiology is herein important: namely that coupled (ATP production) and uncoupled mitochondrial respiration coincides with heat release. Several components of energy expenditure may contribute to heat production, including shivering thermogenesis, non-shivering thermogenesis, diet induced thermogenesis and activity-induced thermogenesis.

Heat production through muscle shivering thermogenesis is a well-known response to acute cold exposure in humans and includes involuntary muscle contractions. Brown adipose tissue (BAT) is responsible for the major part of non-shivering thermogenesis during cold exposure in cold-acclimated small mammals (57). BAT is a tissue enriched with a specific uncoupling protein 1 (UCP-1). In response to cold stimulus, this protein is able to uncouple the proton gradients across the inner mitochondrial membrane and thereby generating heat rather than ATP. Since the thermogenic contribution of BAT is much larger in newborns and small mammals, compared to adult humans, it was for a long time believed that non-shivering thermogenesis through BAT only had a minor contribution to thermogenesis in adult humans (58). However, since the rediscovery of the presence of cold-inducible BAT in adult humans in 2009 (59), activation of BAT has been considered a potential strategy to increase human energy expenditure.

The volume and activity of human BAT are inversely related to body fat content and decrease with age (60). It is therefore expected that the reactivation and recruitment of BAT may protect against the onset of obesity and related metabolic disorders such as T2DM. Cold exposure is the most powerful and physiological stimulus for BAT activation, both in small rodents and in humans (61). In fact, research revealed that acute cold exposure in humans increases whole body resting energy expenditure and improves insulin sensitivity, accompanied by elevated BAT activity (62). Acute cold exposure (hours) increases BAT activity (59, 63-66), while longer-term exposure (days/weeks) can also trigger BAT volume expansion $(67,68)$. In case people are exposed to cold under laboratory conditions for a sustained period, this is referred to as cold acclimation. Cold acclimation studies have reported increased BAT volume as well as elevated BAT activity (69). Furthermore, seasonal variations in basal metabolic rate have been reported with higher values during winter- compared to summertime (70). However, not all beneficial effects of cold exposure on human 
metabolism can fully be explained by BAT activation and hence brings up the question if BAT is the only site of non-shivering thermogenesis. Some studies have proposed that, in addition to shivering, skeletal muscle could also play a role in nonshivering thermogenesis (71-74). A cold acclimation study in patients with T2DM has reported improved skeletal muscle glucose uptake and insulin sensitivity (72). However, the molecular underlying causes are not fully understood yet.

\section{Reducing sedentary behaviour}

Another effective strategy to increase energy expenditure is exercise, which improves insulin sensitivity and mitochondrial function, as previously described. Exercise, however, should not be confused with physical activity. Physical activity is defined as "any bodily movement produced by skeletal muscles that requires energy expenditure" (75). Exercise is a subcategory of physical activity and is defined as "a subset of physical activities that is planned, structured, repetitive and has as a final or an intermediate objective to improve or maintain physical fitness" (75). Physical activity can be subdivided based on intensities expressed in metabolic equivalents (METs). MET is the ratio of energy expenditure during a certain activity to the energy expenditure in rest. One MET is defined as $1 \mathrm{kcal} / \mathrm{kg} / \mathrm{hour}$ and is roughly equivalent to the energy cost of sitting quietly (76). Low-intensity physical activity is defined as activities between 1.5 and 2.9 METs and includes daily activities such as standing, light-intensity walking and light-intensity household activities. Moderate-to-vigorous physical activity is defined as activities $\geq 3.0$ METs and includes activities such as brisk walking, cycling, heavy household work, and exercise. The WHO advises to perform $150 \mathrm{~min} /$ week of moderate-to-vigorous physical activity (77). Worldwide, 31\% of the adult population did not meet this recommendation in 2008, with women being more physically inactive (34\%) than men (28\%) (78). Data obtained in 2016 revealed that $81 \%$ of children aged between 11 and 17 years worldwide were insufficiently physically active in 2016 (79). This indicates that the problem of low physical activity levels will remain a major health problem in the future.

This problem of low physical levels is partly due to an increase in sedentary behaviour. Sedentary behaviour is defined as "any waking behaviour characterized by an energy expenditure $\leq 1.5 \mathrm{METS}$, while in a sitting, reclining or lying posture" (80). Examples of sedentary behaviour include tv viewing, gaming, reading, desk work and driving a car. Sedentary behaviour has frequently been associated with obesity (81) and many chronic diseases including T2DM $(82,83)$, cardiovascular diseases $(82,84)$, and cancer (82). Furthermore, sedentary behaviour has been associated with higher mortality rates (85). Adults in the Netherlands spend on average 35\% of their time 


\section{Chapter 1}

sleeping, $40 \%$ sedentary and only $25 \%$ physically active (86). Compared to healthy individuals, the amount of time spend sedentary is higher in the obese population and even higher in patients with T2DM (86).

One of the questions that remains to be answered is whether sitting time should be replaced by low-intensity physical activities such as standing and walking, or by moderate-to-vigorous physical activities such as exercise. It has already been shown that replacement of sitting time by standing and walking, as well as by exercise, improves glucose and lipid metabolism, including markers of insulin sensitivity (8789). Whether these two sitting replacement strategies act via the same underlying pathway is still unknown.
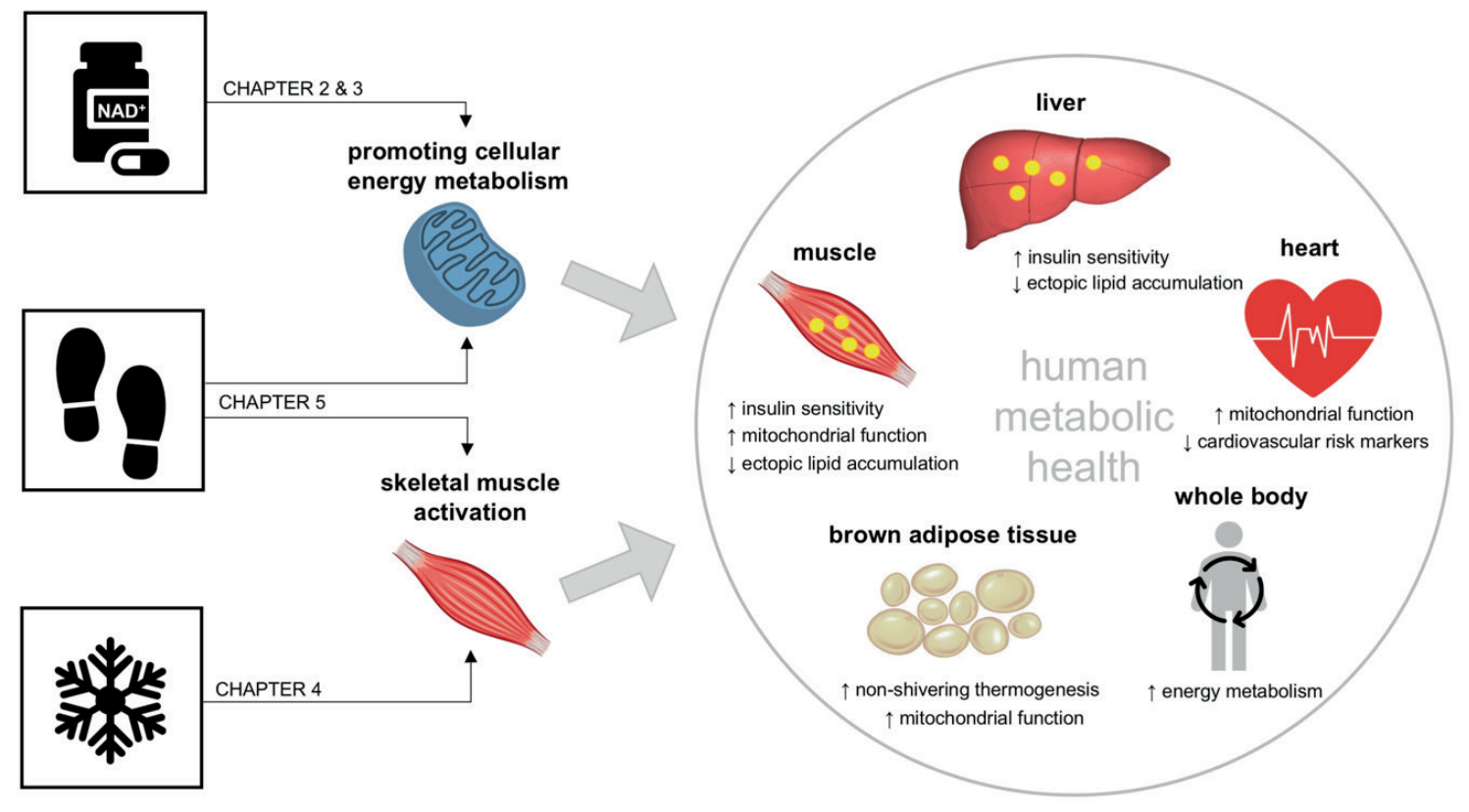

Figure 2. Proposed working mechanism of novel strategies to improve human metabolic health 


\section{OUTLINE OF THIS THESIS}

The aim of this thesis is to investigate novel strategies to improve human metabolic health, affecting insulin sensitivity and mitochondrial function. Three human intervention studies have been performed which line up the different chapters of this thesis (see Figure 2). In chapter 2, the effect of nicotinamide riboside supplementation - a NAD+ precursor - on human metabolic health is described, with a focus on insulin sensitivity and muscle mitochondrial function. Chapter 3 describes the effect of nicotinamide riboside on brown adipose tissue activity. Chapter 4 of this thesis addresses the effect of cold acclimation on human insulin sensitivity and postprandial substrate metabolism. In chapter 5 , the effect of replacing sedentary behavior by standing and walking compared to a single exercise bout per day are presented with respect to human insulin sensitivity and muscle mitochondrial function. In chapter 6 , all findings of the previous parts will be discussed, and overall conclusions of this thesis will be drawn. 


\section{REFERENCES}

1. WHO. Factsheet obesity and overweight 2018 [Available from: https://www.who.int/newsroom/fact-sheets/detail/obesity-and-overweight.

2. RIVM. Overgewicht cijfers en context 2018 [Available from: https://www.volksgezondheidenzorg.info/onderwerp/overgewicht/cijfers-

context/samenvatting\#node-overgewicht-samengevat.

3. Kopelman P. Health risks associated with overweight and obesity. Obes Rev. 2007;8 Suppl 1:13-7.

4. WHO. Top 10 causes of death 2018 [Available from: https://www.who.int/gho/mortality_burden_disease/causes_death/top_10/en/.

5. Pineda E, Sanchez-Romero LM, Brown M, Jaccard A, Jewell J, Galea G, et al. Forecasting Future Trends in Obesity across Europe: The Value of Improving Surveillance. Obes Facts. 2018;11(5):360-71.

6. WHO. Factsheet diabetes 2018 [Available from: https://www.who.int/news-room/factsheets/detail/diabetes.

7. RIVM. Diabetes mellitus cijfers en context 2018 [Available from: https://www.volksgezondheidenzorg.info/onderwerp/diabetes-mellitus/cijfers-context/huidigesituatie.

8. RIVM. Ranglijst aandoeningen op basis van ziektelast (in DALY's) 2018 [Available from: https://www.volksgezondheidenzorg.info/ranglijst/ranglijst-aandoeningen-op-basis-vanziektelast-dalys.

9. RIVM. Volksgezondheid toekomst verkenning 2018 [Available from: https://www.vtv2018.nl/aandoeningen.

10. Cersosimo E, Triplitt C, Solis-Herrera C, Mandarino LJ, DeFronzo RA. Pathogenesis of Type 2 Diabetes Mellitus. In: Feingold KR, Anawalt B, Boyce A, Chrousos G, Dungan K, Grossman A, et al., editors. Endotext. South Dartmouth (MA)2000.

11. DeFronzo RA, Gunnarsson R, Bjorkman O, Olsson M, Wahren J. Effects of insulin on peripheral and splanchnic glucose metabolism in noninsulin-dependent (type II) diabetes mellitus. The Journal of clinical investigation. 1985;76(1):149-55.

12. Wojtaszewski JF, Hansen BF, Kiens B, Richter EA. Insulin signaling in human skeletal muscle: time course and effect of exercise. Diabetes. 1997;46(11):1775-81.

13. Kennedy JW, Hirshman MF, Gervino EV, Ocel JV, Forse RA, Hoenig SJ, et al. Acute exercise induces GLUT4 translocation in skeletal muscle of normal human subjects and subjects with type 2 diabetes. Diabetes. 1999;48(5):1192-7.

14. Shulman Gl. Ectopic fat in insulin resistance, dyslipidemia, and cardiometabolic disease. N Engl J Med. 2014;371(12):1131-41.

15. Deshpande AD, Harris-Hayes M, Schootman M. Epidemiology of diabetes and diabetesrelated complications. Phys Ther. 2008;88(11):1254-64.

16. Rolo AP, Palmeira CM. Diabetes and mitochondrial function: role of hyperglycemia and oxidative stress. Toxicol Appl Pharmacol. 2006;212(2):167-78.

17. Szendroedi J, Roden M. Ectopic lipids and organ function. Curr Opin Lipidol. 2009;20(1):50-6.

18. Seo AY, Joseph AM, Dutta D, Hwang JC, Aris JP, Leeuwenburgh C. New insights into the role of mitochondria in aging: mitochondrial dynamics and more. J Cell Sci. 2010;123(Pt 15):253342.

19. Chistiakov DA, Sobenin IA, Revin VV, Orekhov AN, Bobryshev YV. Mitochondrial aging and age-related dysfunction of mitochondria. Biomed Res Int. 2014;2014:238463.

20. Ballinger SW. Mitochondrial dysfunction in cardiovascular disease. Free radical biology \& medicine. 2005;38(10):1278-95.

21. Kelley DE, He J, Menshikova EV, Ritov VB. Dysfunction of mitochondria in human skeletal muscle in type 2 diabetes. Diabetes. 2002;51(10):2944-50.

22. Sigal RJ, Kenny GP, Wasserman DH, Castaneda-Sceppa C. Physical activity/exercise and type 2 diabetes. Diabetes care. 2004;27(10):2518-39.

23. Yerramalla MS, Fayosse A, Dugravot A, Tabak AG, Kivimaki M, Singh-Manoux A, et al. Association of moderate and vigorous physical activity with incidence of type 2 diabetes and subsequent mortality: 27 year follow-up of the Whitehall II study. Diabetologia. 2019.

24. Xue B, Kahn BB. AMPK integrates nutrient and hormonal signals to regulate food intake and energy balance through effects in the hypothalamus and peripheral tissues. The Journal of physiology. 2006;574(Pt 1):73-83. 
25. Kemp BE, Mitchelhill KI, Stapleton D, Michell BJ, Chen ZP, Witters LA. Dealing with energy demand: the AMP-activated protein kinase. Trends in biochemical sciences. 1999;24(1):22-5.

26. Boutant M, Canto C. SIRT1 metabolic actions: Integrating recent advances from mouse models. Mol Metab. 2014;3(1):5-18.

27. Barger JL, Walford RL, Weindruch $\mathrm{R}$. The retardation of aging by caloric restriction: its significance in the transgenic era. Experimental gerontology. 2003;38(11-12):1343-51.

28. Heilbronn LK, de Jonge L, Frisard MI, DeLany JP, Larson-Meyer DE, Rood J, et al. Effect of 6month calorie restriction on biomarkers of longevity, metabolic adaptation, and oxidative stress in overweight individuals: a randomized controlled trial. Jama. 2006;295(13):1539-48.

29. Civitarese AE, Carling S, Heilbronn LK, Hulver MH, Ukropcova B, Deutsch WA, et al. Calorie restriction increases muscle mitochondrial biogenesis in healthy humans. PLoS Med. 2007;4(3):e76.

30. Larson-Meyer DE, Heilbronn LK, Redman LM, Newcomer BR, Frisard MI, Anton S, et al. Effect of calorie restriction with or without exercise on insulin sensitivity, beta-cell function, fat cell size, and ectopic lipid in overweight subjects. Diabetes care. 2006;29(6):1337-44.

31. Goodpaster BH, Kelley DE, Wing RR, Meier A, Thaete FL. Effects of weight loss on regional fat distribution and insulin sensitivity in obesity. Diabetes. 1999;48(4):839-47.

32. Toledo FG, Menshikova EV, Ritov VB, Azuma K, Radikova Z, DeLany J, et al. Effects of physical activity and weight loss on skeletal muscle mitochondria and relationship with glucose control in type 2 diabetes. Diabetes. 2007;56(8):2142-7.

33. Meex RC, Schrauwen-Hinderling VB, Moonen-Kornips E, Schaart G, Mensink M, Phielix E, et al. Restoration of muscle mitochondrial function and metabolic flexibility in type 2 diabetes by exercise training is paralleled by increased myocellular fat storage and improved insulin sensitivity. Diabetes. 2010;59(3):572-9.

34. Phielix E, Meex R, Moonen-Kornips E, Hesselink MK, Schrauwen P. Exercise training increases mitochondrial content and ex vivo mitochondrial function similarly in patients with type 2 diabetes and in control individuals. Diabetologia. 2010;53(8):1714-21.

35. Brouwers B, Schrauwen-Hinderling VB, Jelenik T, Gemmink A, Sparks LM, Havekes B, et al. Exercise training reduces intrahepatic lipid content in people with and people without nonalcoholic fatty liver. Am J Physiol Endocrinol Metab. 2018;314(2):E165-E73.

36. Pesta D, Hoppel F, Macek C, Messner H, Faulhaber M, Kobel C, et al. Similar qualitative and quantitative changes of mitochondrial respiration following strength and endurance training in normoxia and hypoxia in sedentary humans. Am J Physiol Regul Integr Comp Physiol. 2011;301(4):R1078-87.

37. Sogaard D, Lund MT, Scheuer CM, Dehlbaek MS, Dideriksen SG, Abildskov CV, et al. Highintensity interval training improves insulin sensitivity in older individuals. Acta Physiol (Oxf). 2018;222(4):e13009.

38. Richter EA, Hargreaves M. Exercise, GLUT4, and skeletal muscle glucose uptake. Physiol Rev. 2013;93(3):993-1017.

39. Mikines KJ, Sonne B, Farrell PA, Tronier B, Galbo H. Effect of physical exercise on sensitivity and responsiveness to insulin in humans. Am J Physiol. 1988;254(3 Pt 1):E248-59.

40. Gibson AA, Sainsbury A. Strategies to Improve Adherence to Dietary Weight Loss Interventions in Research and Real-World Settings. Behav Sci (Basel). 2017;7(3).

41. Morrato EH, Hill JO, Wyatt HR, Ghushchyan V, Sullivan PW. Physical activity in U.S. adults with diabetes and at risk for developing diabetes, 2003. Diabetes care. 2007;30(2):203-9.

42. Houtkooper $\mathrm{RH}$, Canto C, Wanders RJ, Auwerx J. The secret life of NAD+: an old metabolite controlling new metabolic signaling pathways. Endocr Rev. 2010;31(2):194-223.

43. Canto C, Gerhart-Hines Z, Feige JN, Lagouge M, Noriega L, Milne JC, et al. AMPK regulates energy expenditure by modulating NAD+ metabolism and SIRT1 activity. Nature. 2009;458(7241): 1056-60.

44. Houtkooper RH, Pirinen E, Auwerx J. Sirtuins as regulators of metabolism and healthspan. Nat Rev Mol Cell Biol. 2012;13(4):225-38.

45. Taskinen MR, Nikkila EA. Effects of acipimox on serum lipids, lipoproteins and lipolytic enzymes in hypertriglyceridemia. Atherosclerosis. 1988;69(2-3):249-55.

46. van de Weijer T, Phielix E, Bilet L, Williams EG, Ropelle ER, Bierwagen A, et al. Evidence for a direct effect of the NAD+ precursor acipimox on muscle mitochondrial function in humans. Diabetes. 2015;64(4):1193-201.

47. Benyo Z, Gille A, Kero J, Csiky M, Suchankova MC, Nusing RM, et al. GPR109A (PUMA$\mathrm{G} / \mathrm{HM} 74 \mathrm{~A})$ mediates nicotinic acid-induced flushing. The Journal of clinical investigation. 2005;115(12):3634-40. 
48. Connell NJ, Houtkooper $\mathrm{RH}$, Schrauwen P. NAD(+) metabolism as a target for metabolic health: have we found the silver bullet? Diabetologia. 2019;62(6):888-99.

49. Yoshino J, Mills KF, Yoon MJ, Imai S. Nicotinamide mononucleotide, a key $N A D(+)$ intermediate, treats the pathophysiology of diet- and age-induced diabetes in mice. Cell metabolism. 2011;14(4):528-36.

50. Canto C, Houtkooper RH, Pirinen E, Youn DY, Oosterveer MH, Cen Y, et al. The NAD(+) precursor nicotinamide riboside enhances oxidative metabolism and protects against high-fat diet-induced obesity. Cell metabolism. 2012;15(6):838-47.

51. Khan NA, Auranen M, Paetau I, Pirinen E, Euro L, Forsstrom S, et al. Effective treatment of mitochondrial myopathy by nicotinamide riboside, a vitamin B3. EMBO molecular medicine. 2014;6(6):721-31.

52. Trammell SA, Weidemann BJ, Chadda A, Yorek MS, Holmes A, Coppey LJ, et al. Nicotinamide Riboside Opposes Type 2 Diabetes and Neuropathy in Mice. Sci Rep. 2016;6:26933.

53. Shi W, Hegeman MA, van Dartel DAM, Tang J, Suarez M, Swarts H, et al. Effects of a wide range of dietary nicotinamide riboside (NR) concentrations on metabolic flexibility and white adipose tissue (WAT) of mice fed a mildly obesogenic diet. Mol Nutr Food Res. 2017;61(8).

54. Trammell SA, Schmidt MS, Weidemann BJ, Redpath P, Jaksch F, Dellinger RW, et al. Nicotinamide riboside is uniquely and orally bioavailable in mice and humans. Nature communications. 2016;7:12948.

55. Dellinger RW, Santos SR, Morris M, Evans M, Alminana D, Guarente L, et al. Erratum: Author Correction: Repeat dose NRPT (nicotinamide riboside and pterostilbene) increases NAD $(+)$ levels in humans safely and sustainably: a randomized, double-blind, placebo-controlled study. NPJ Aging Mech Dis. 2017;4:8.

56. Airhart SE, Shireman LM, Risler LJ, Anderson GD, Nagana Gowda GA, Raftery D, et al. An open-label, non-randomized study of the pharmacokinetics of the nutritional supplement nicotinamide riboside (NR) and its effects on blood NAD+ levels in healthy volunteers. PloS one. 2017;12(12):e0186459.

57. Daanen HA, Van Marken Lichtenbelt WD. Human whole body cold adaptation. Temperature (Austin). 2016;3(1):104-18.

58. Marlatt KL, Ravussin E. Brown Adipose Tissue: an Update on Recent Findings. Curr Obes Rep. 2017;6(4):389-96.

59. van Marken Lichtenbelt WD, Vanhommerig JW, Smulders NM, Drossaerts JM, Kemerink GJ, Bouvy ND, et al. Cold-activated brown adipose tissue in healthy men. $\mathrm{N}$ Engl J Med. 2009;360(15):1500-8.

60. Hanssen MJ, van der Lans AA, Brans B, Hoeks J, Jardon KM, Schaart G, et al. Short-term Cold Acclimation Recruits Brown Adipose Tissue in Obese Humans. Diabetes. 2016;65(5):1179-89.

61. Peres Valgas da Silva C, Hernandez-Saavedra D, White JD, Stanford KI. Cold and Exercise: Therapeutic Tools to Activate Brown Adipose Tissue and Combat Obesity. Biology (Basel). 2019;8(1).

62. Chondronikola M, Volpi E, Borsheim E, Porter C, Annamalai P, Enerback S, et al. Brown adipose tissue improves whole-body glucose homeostasis and insulin sensitivity in humans. Diabetes. 2014;63(12):4089-99.

63. Cypess AM, Lehman S, Williams G, Tal I, Rodman D, Goldfine AB, et al. Identification and importance of brown adipose tissue in adult humans. N Engl J Med. 2009;360(15):1509-17.

64. Saito M, Okamatsu-Ogura Y, Matsushita M, Watanabe K, Yoneshiro T, Nio-Kobayashi J, et al. High incidence of metabolically active brown adipose tissue in healthy adult humans: effects of cold exposure and adiposity. Diabetes. 2009;58(7):1526-31.

65. Virtanen KA, Lidell ME, Orava J, Heglind M, Westergren R, Niemi T, et al. Functional brown adipose tissue in healthy adults. N Engl J Med. 2009;360(15):1518-25.

66. Chen KY, Brychta RJ, Linderman JD, Smith S, Courville A, Dieckmann W, et al. Brown fat activation mediates cold-induced thermogenesis in adult humans in response to a mild decrease in ambient temperature. J Clin Endocrinol Metab. 2013;98(7):E1218-23.

67. van der Lans AA, Hoeks J, Brans B, Vijgen GH, Visser MG, Vosselman MJ, et al. Cold acclimation recruits human brown fat and increases nonshivering thermogenesis. The Journal of clinical investigation. 2013;123(8):3395-403.

68. Yoneshiro T, Aita S, Matsushita M, Kayahara T, Kameya T, Kawai Y, et al. Recruited brown adipose tissue as an antiobesity agent in humans. The Journal of clinical investigation. 2013;123(8):3404-8.

69. Blondin DP, Labbe SM, Tingelstad HC, Noll C, Kunach M, Phoenix S, et al. Increased brown adipose tissue oxidative capacity in cold-acclimated humans. J Clin Endocrinol Metab. 2014;99(3):E438-46. 
70. Plasqui $G$, Kester AD, Westerterp KR. Seasonal variation in sleeping metabolic rate, thyroid activity, and leptin. Am J Physiol Endocrinol Metab. 2003;285(2):E338-43.

71. Wijers SLJ, Schrauwen P, Saris WHM, Lichtenbelt WDV. Human Skeletal Muscle Mitochondrial Uncoupling Is Associated with Cold Induced Adaptive Thermogenesis. PloS one. 2008;3(3).

72. Hanssen MJ, Hoeks J, Brans B, van der Lans AA, Schaart G, van den Driessche JJ, et al. Shortterm cold acclimation improves insulin sensitivity in patients with type 2 diabetes mellitus. Nat Med. 2015;21(8):863-5.

73. Blondin DP, Daoud A, Taylor T, Tingelstad HC, Bezaire V, Richard D, et al. Four-week cold acclimation in adult humans shifts uncoupling thermogenesis from skeletal muscles to brown adipose tissue. The Journal of physiology. 2017;595(6):2099-113.

74. Gordon K, Blondin DP, Friesen BJ, Tingelstad HC, Kenny GP, Haman F. Seven days of cold acclimation substantially reduces shivering intensity and increases nonshivering thermogenesis in adult humans. J Appl Physiol (1985). 2019;126(6):1598-606.

75. Caspersen CJ, Powell KE, Christenson GM. Physical activity, exercise, and physical fitness: definitions and distinctions for health-related research. Public Health Rep. 1985;100(2):126-31.

76. Ainsworth BE, Haskell WL, Whitt MC, Irwin ML, Swartz AM, Strath SJ, et al. Compendium of physical activities: an update of activity codes and MET intensities. Med Sci Sports Exerc. 2000;32(9 Suppl):S498-504.

77. WHO. Global Recommendations on Physical Activity for Health. 2011.

78. WHO. Factsheet physical inactivity: a global public health problem 2008 [Available from: https://www.who.int/dietphysicalactivity/factsheet_inactivity/en/.

79. Guthold R, Stevens GA, Riley LM, Bull FC. Global trends in insufficient physical activity among adolescents: a pooled analysis of 298 population-based surveys with 1.6 million participants. Lancet Child Adolesc Health. 2020;4(1):23-35.

80. Tremblay MS, Aubert S, Barnes JD, Saunders TJ, Carson V, Latimer-Cheung AE, et al. Sedentary Behavior Research Network (SBRN) - Terminology Consensus Project process and outcome. Int J Behav Nutr Phys Act. 2017;14(1):75.

81. Owen N, Healy GN, Matthews CE, Dunstan DW. Too much sitting: the population health science of sedentary behavior. Exerc Sport Sci Rev. 2010;38(3):105-13.

82. Biswas A, Oh PI, Faulkner GE, Bajaj RR, Silver MA, Mitchell MS, et al. Sedentary time and its association with risk for disease incidence, mortality, and hospitalization in adults: a systematic review and meta-analysis. Ann Intern Med. 2015;162(2):123-32.

83. van der Berg JD, Stehouwer CD, Bosma H, van der Velde JH, Willems PJ, Savelberg HH, et al. Associations of total amount and patterns of sedentary behaviour with type 2 diabetes and the metabolic syndrome: The Maastricht Study. Diabetologia. 2016;59(4):709-18.

84. Powell KE, Thompson PD, Caspersen CJ, Kendrick JS. Physical activity and the incidence of coronary heart disease. Annu Rev Public Health. 1987;8:253-87.

85. van der Ploeg HP, Chey T, Korda RJ, Banks E, Bauman A. Sitting time and all-cause mortality risk in 222497 Australian adults. Arch Intern Med. 2012;172(6):494-500.

86. JD VDB, JHPM VDV, EAC DEW, Bosma H, Savelberg H, Schaper NC, et al. Replacement Effects of Sedentary Time on Metabolic Outcomes: The Maastricht Study. Med Sci Sports Exerc. 2017;49(7):1351-8.

87. Duvivier B, Schaper NC, Koster A, van Kan L, Peters HPF, Adam JJ, et al. Benefits of Substituting Sitting with Standing and Walking in Free-Living Conditions for Cardiometabolic Risk Markers, Cognition and Mood in Overweight Adults. Front Physiol. 2017;8:353.

88. Duvivier BM, Schaper NC, Bremers MA, van Crombrugge G, Menheere PP, Kars M, et al. Minimal intensity physical activity (standing and walking) of longer duration improves insulin action and plasma lipids more than shorter periods of moderate to vigorous exercise (cycling) in sedentary subjects when energy expenditure is comparable. PloS one. 2013;8(2):e55542.

89. Duvivier BM, Schaper NC, Hesselink MK, van Kan L, Stienen N, Winkens B, et al. Breaking sitting with light activities vs structured exercise: a randomised crossover study demonstrating benefits for glycaemic control and insulin sensitivity in type 2 diabetes. Diabetologia. 2016;60(3):490-8. 



\section{CHAPTER 2}

Nicotinamide riboside supplementation alters body composition and skeletal muscle acetylcarnitine concentrations in healthy obese humans

Carlijn M.E. Remie, Kay H.M. Roumans, Michiel P.B. Moonen, Niels J. Connell, Bas Havekes, Julian Mevenkamp, Lucas Lindeboom, Vera H.W. de Wit, Tineke van de Weijer, Suzanne A.B.M. Aarts, Esther Lutgens, Bauke V. Schomakers, Hyung L. Elfrink, Rubén Zapata-Pérez, Riekelt H. Houtkooper, Johan Auwerx, Joris Hoeks, Vera B. Schrauwen-Hinderling, Esther Phielix, Patrick Schrauwen

Am J Clin Nutr. 2020 Apr 22; nqaa072. Online ahead of print.

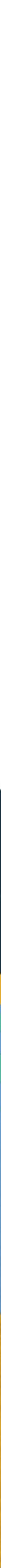




\section{ABSTRACT}

Background: Nicotinamide riboside (NR) is a NAD+ precursor that boosts cellular $\mathrm{NAD}^{+}$concentrations. Preclinical studies have shown profound metabolic health effects after NR supplementation.

Objective: Investigate the effects of six weeks NR supplementation on insulin sensitivity, mitochondrial function and other metabolic health parameters in overweight and obese volunteers.

Design: A randomized, double-blinded, placebo-controlled, cross-over intervention study was conducted in thirteen healthy overweight and obese men and women. Participants received six weeks NR (1000 mg/day) and placebo supplementation, followed by broad metabolic phenotyping, including hyperinsulinemic-euglycemic clamps, magnetic resonance spectroscopy, muscle biopsies, and assessment of ex vivo mitochondrial function and in vivo energy metabolism.

Results: Markers of increased NAD+ synthesis - NAAD and MeNAM - were elevated in skeletal muscle after NR compared to placebo. NR increased body fat free mass (62.65 \pm 2.49 vs. $61.32 \pm 2.58,1.34 \pm 0.50 \%$ in NR, placebo, and the change respectively, $\mathrm{p}=0.02$ ) and increased sleeping metabolic rate. Interestingly, acetylcarnitine concentrations in skeletal muscle were increased upon NR (4558 \pm 749 vs. $3025 \pm 316$ and $1533 \pm 683 \mathrm{pmol} / \mathrm{mg}$ dry weight in NR, placebo, and the change respectively, $\mathrm{p}=0.04$ ) and the capacity to form acetylcarnitine upon exercise was higher in NR compared to placebo $(2.99 \pm 0.30$ vs. $2.40 \pm 0.33$ and $0.53 \pm 0.21$ $\mathrm{mmol} / \mathrm{kg}$ wet weight in $\mathrm{NR}$, placebo, and the change respectively, $\mathrm{p}=0.01$ ). However, no effects of NR were found on insulin sensitivity, mitochondrial function, hepatic and intramyocellular lipid accumulation, cardiac energy status, cardiac ejection fraction, ambulatory blood pressure, plasma markers of inflammation or energy metabolism.

Conclusion: NR supplementation of $1000 \mathrm{mg} /$ day for six weeks in healthy overweight and obese men and women increased skeletal muscle NAD+ metabolites, affected skeletal muscle acetylcarnitine metabolism, and induced minor changes in body composition and sleeping metabolic rate. However, no other metabolic health effects were observed. 


\section{INTRODUCTION}

Nicotinamide riboside (NR) is a naturally occurring vitamin B3 present in the human diet, acts as a NAD+ precursor (1), and is suggested to improve mitochondrial function and insulin sensitivity (2). NR acts via activation of the NAD+-dependent sirtuin enzyme family, thereby regulating oxidative metabolism (3-7). In vitro experiments have shown the successful $\mathrm{NAD}^{+}$restoring capability of NR supplementation and the subsequent increase oxidative gene expression in skeletal muscle cells $(8,9)$. Results from in vivo mouse models have shown improvements in insulin sensitivity and oxidative energy metabolism, including enhanced metabolic flexibility, increased aerobic exercise capacity and indications of improved mitochondrial biogenesis (913). In these mouse models NR seems to specifically act on muscle, liver, heart and brown adipose tissue $(9,14)$. Reports indicate that $\mathrm{NAD}^{+}$metabolism including $N A D^{+}$ concentrations are decreased in the obese and older population (15-17). The number of human interventions with NR is small and the evidence that NR may have beneficial effects in humans is limited. Human pharmacokinetic studies showed increased circulatory $\mathrm{NAD}^{+}$metabolite concentrations in whole blood, $\mathrm{PBMC}$ and urine, after various dosages of NR supplementation (18-23), varying from 300 to $2000 \mathrm{mg} / \mathrm{day}$, indicating that NR supplementation is able to increase the NAD+ pool in humans. So far, three randomized placebo-controlled NR supplementation studies have been performed in humans, in which the effect of NR supplementation on human metabolic health was investigated. Dollerup et al. (21, 24), investigated the effect of 2000 $\mathrm{mg} /$ day NR for 12 weeks in insulin resistant middle-aged obese men. No effects of NR supplementation were found on insulin sensitivity, muscle mitochondrial function or metabolic flexibility $(21,24)$. In addition, Martens et al. (20), investigated the effect of $1000 \mathrm{mg} /$ day NR for six weeks in healthy normal weight middle-aged and older men and women. Besides a trend in a reduction in a reduced arterial stiffness and lower blood pressure after NR supplementation, no effects were found on a wide variety of outcomes indicative of metabolic function, glucose metabolism, motor function and exercise capacity (20). Furthermore, Elhassan et al. (25) investigated the effects of $1000 \mathrm{mg} /$ day NR for 3 weeks in older men and showed increased skeletal muscle $\mathrm{NAD}^{+}$metabolite concentrations, however again no effect on skeletal muscle mitochondrial function was observed. To date, the effect of NR supplementation on both insulin sensitivity and skeletal muscle mitochondrial function has only been recently published in one human clinial trial $(21,24)$. Based on the promising preclinical findings reported until 2017, we designed a double blinded randomized placebo controlled cross-over study in which we aimed to investigate the effect of $1000 \mathrm{mg} /$ day NR supplementation on metabolic health in healthy obese and overweight men and women. The primary focus was on insulin sensitivity and muscle 
mitochondrial function, and secondary outcomes were related to energy metabolism and skeletal muscle $\mathrm{NAD}^{+}$metabolites. Therefore, our study expands the limited knowledge of the effect of NR supplementation on human metabolic health and aims to investigate the translational value of previous promising preclinical findings.

\section{METHODS}

The study was conducted in accordance with the principles of the declaration of Helsinki and approved by the Ethics Committee of the Maastricht University Medical Center. The study was registered at https://clinicaltrials.gov (NCT02835664). All participants provided written informed consent before screening.

\section{Participants}

Recruitment and data collection took place between December 2016 and December 2018 in Maastricht, The Netherlands. Thirteen participants completed the study, two dropped out because of personal or medical reasons (see Supplementary Figure 1). Men and postmenopausal women were included. Participants underwent a screening including assessment of blood biochemistry, electrocardiography, anthropometric measurements and a questionnaire (including Baecke physical activity questionnaire (26)) to evaluate eligibility. Inclusion criteria were 45-65 years of age, BMI 27-35 $\mathrm{kg} / \mathrm{m}^{2}$, sedentary lifestyle ( $<3 \mathrm{~h}$ exercise per week), non-smoking for at least 6 months, alcohol use of $\leq 2$ servings per day, stable body weight for at least 6 months and having no active diseases.

\section{Study design}

The study had a randomized controlled, double blinded, placebo controlled, crossover design. Simple randomization was applied with use of computer-generated random numbers. Each participant received two interventions each of which lasted six weeks. During the first six weeks the participant was randomized to receive either NR supplementation or placebo. This six-week period was followed by a washout period of four to seven weeks. At the end of the washout period the participant crossed over to the treatment they did not receive during the first six weeks. In both intervention arms participants underwent exactly the same tests to determine the effect of the intervention (Figure 1). These tests were performed in the end of week five and during week six. During the entire study period no changes in lifestyle (no change in diet, physical activity level or medication or supplement use) were allowed. Compliance was checked on a weekly basis by pill count. Furthermore, a fasted blood 
sample was taken, and body weight was monitored. During supplementation periods, any adverse effects and side effects were noted.

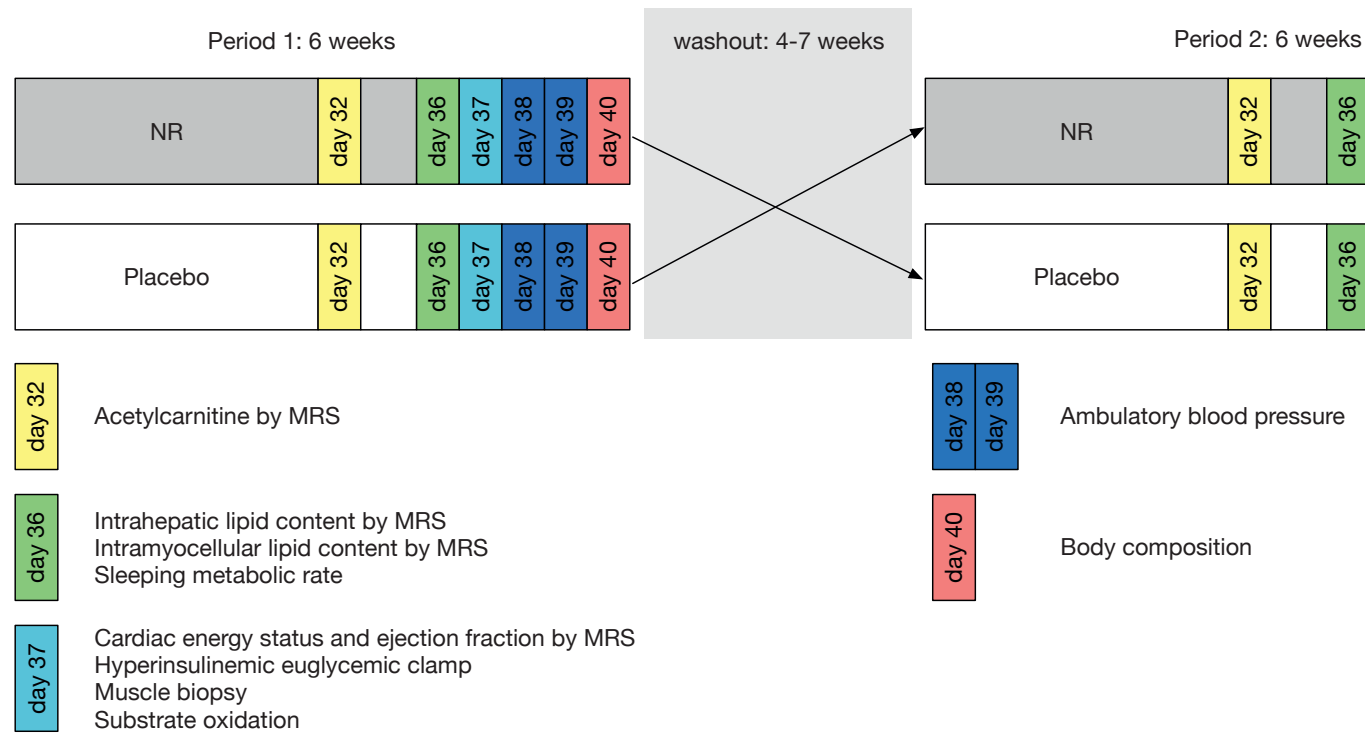

\section{Figure 1. Study design}

In this cross-over study, participants were randomly assigned to start with six weeks of NR supplementation or six weeks of placebo treatment. After a washout period of four-to-seven weeks, participants entered the other intervention arm such that all participants served as their own control. Participants were studied in week five (day 32) and in week six (day 36 - 40) of each of the two interventions.

\section{Determination of sleeping metabolic rate}

At day 36 of each intervention, participants received a standardized dinner at 18:30 $\mathrm{h}$ after which they remained fasted and entered a metabolic chamber at 19:30 h. The metabolic chamber is a small room with a bed, toilet, television, computer and access to water in which oxygen consumption and carbohydrate production were measured continuously in sampled room air. During the overnight stay in the respiration chamber sleeping metabolic rate was assessed. Participants decided themselves what time they went to sleep. At 06:00 h the next morning participants were woken up and left the chamber in an overnight fasted state.

\section{Hyperinsulinemic euglycemic clamp}

To determine insulin sensitivity, a two-step hyperinsulinemic euglycemic clamp with co-infusion of D-[6.6-2 $\left.{ }^{2} \mathrm{H}_{2}\right]$ glucose tracer $(0.04 \mathrm{mg} / \mathrm{kg} / \mathrm{min})$ was performed on day 37 of both interventions (27). Insulin was infused, starting at $10: 30 \mathrm{~h}$, at $10 \mathrm{mU} / \mathrm{m}^{2} / \mathrm{min}$ for $2.5 \mathrm{~h}$ to assess hepatic insulin sensitivity and subsequently increased to 40 
$\mathrm{mU} / \mathrm{m}^{2} / \mathrm{min}$ for $2 \mathrm{~h}$ to measure whole body insulin sensitivity. Blood was frequently sampled to measure glucose concentrations directly from arterialized blood and tracer-enriched $20 \%$ glucose was co-infused at a variable rate to maintain euglycemia ( $\sim 5.0 \mathrm{mmol} / \mathrm{l})$ and reach steady state condition. During the steady state of 30 minutes, blood samples were collected, and substrate utilization was measured using indirect calorimetry. Due to technical failures, one participant was excluded from the clamp analysis.

\section{Skeletal muscle biopsies}

On day 37 of each intervention, a muscle biopsy was taken at 8:30 h after an overnight stay in the respiration chamber from the $\mathrm{m}$. vastus lateralis under local anesthesia (1\% lidocaine, without epinephrine) using the Bergström technique (28). The muscle biopsy was divided in several parts. One part was immediately frozen in melting isopentane for biochemical analyses. The remaining part was used for mitochondrial respiration analysis.

\section{Skeletal muscle $\mathrm{NAD}^{+}$metabolites}

$\mathrm{NAD}^{+}$content was determined in muscle biopsies using an enzymatic spectrophotometric cycling assay based on the coupled reaction of malate and alcohol dehydrogenases, as previously described (29). NAD ${ }^{+}$metabolites were measured in muscle biopsies through metabolomics. Freeze-dried muscle tissue (2$4 \mathrm{mg}$ ) was transferred to $2 \mathrm{~mL}$ tubes, and then $425 \mu \mathrm{L}$ water, $500 \mu \mathrm{L}$ methanol and 75 $\mu \mathrm{L}$ of internal standards mixture (see Supplementary Table 1) was added to each sample. Samples were homogenized using TissueLyser II (Qiagen; 5 min at 30/s), followed by addition of $1000 \mu \mathrm{L}$ chloroform and thorough mixing. After centrifugation, the top layer containing the polar phase was transferred to $1.5 \mathrm{~mL}$ tubes and dried in a vacuum evaporator at $60{ }^{\circ} \mathrm{C}$. Dried samples were reconstituted in $100 \mu \mathrm{L}$ methanol/water (6/4, v/v) and analyzed in an Aquity UPLC system (Waters) coupled to an Impact IITM Ultra-High Resolution qTOF mass spectrometer (Bruker). Chromatographic separation of the compounds was achieved using a SeQuant ZICcHILIC column (PEEK $100 \times 2.1 \mathrm{~mm}, 3 \mu \mathrm{m}$ particle size, Merck, Darmstadt, Germany) at $30{ }^{\circ} \mathrm{C}$. The LC method consisted in a gradient running at $0.25 \mathrm{~mL} / \mathrm{min}$ from $100 \%$ mobile phase B (9:1 acetonitrile:water with $5 \mathrm{mM}$ ammonium acetate $\mathrm{pH} 6.8)$ to 100\% mobile phase A (1:9 acetonitrile:water with $5 \mathrm{mM}$ ammonium acetate $\mathrm{pH} 6.8$ ) in 28 minutes, followed by a re-equilibration step at 100\% B of 5 minutes. MS data were acquired both in negative and positive ionization modes in full scan mode over the range of $\mathrm{m} / \mathrm{z}$ 50-1200. Due to limited sample availability, $\mathrm{NAD}^{+}$content was measured in muscle biopsies of 8 participants and NAD+ metabolomics was measured in muscle biopsies of 12 participants. 


\section{Skeletal muscle mitochondrial respiration and protein content}

From the muscle biopsies, permeabilized muscle fibers were prepared as described elsewhere (30). Thereafter ex vivo mitochondrial respiration was determined by measuring oxygen consumption rate upon addition of several substrates using highresolution respirometry (Oxygraph, OROBOROS Instruments, Innsbruck, Austria) as described previously (31). All measurements were performed in quadruplicate and the integrity of the outer mitochondrial membrane was assessed in every experiment by the addition of cytochrome $\mathrm{C}(10 \mu \mathrm{mol} / \mathrm{L})$ upon maximal coupled respiration. The average cytochrome $\mathrm{C}$ response in the included traces was $1.7 \pm 0.03 \%$ and traces with a cytochrome $\mathrm{C}$ response above $15 \%$ were excluded from statistical analyses. Data is presented per mg wet weight. Oxphos complex protein content was measured in skeletal muscle biopsies of 12 participants as previously described (32).

\section{Skeletal muscle acylcarnitine concentrations}

2-4 $\mathrm{mg}$ of freeze-dried muscle tissue was homogenized $1 \mathrm{~mL} 80 \%$ acetonitrile containing $50 \mu \mathrm{L}$ of the internal standards. After centrifugation at $16000 \mathrm{~g}$, the resulting supernatant was dried under a stream of nitrogen at $40^{\circ} \mathrm{C}$ and derivatized by addition of 1-propanol/acetylchloride $(4 / 1, \mathrm{v} / \mathrm{v})$ during 15 minutes at $60^{\circ} \mathrm{C}$. After evaporation under nitrogen at $40{ }^{\circ} \mathrm{C}$, samples were redissolved in pure acetonitrile. Determination of the propylated acylcarnitines in the medium was performed by mass spectrometry in an Acquity UPLC System (Waters) coupled to a Quattro Premier XE Tandem Quadrupole Mass Spectrometer (Waters).

\section{$\mathrm{VO}_{2}$ peak}

Maximal oxygen consumption ( $\mathrm{VO}_{2}$ peak) was assessed during an incremental cycling test on an ergometer (Lode Excalibur Sport, Groningen, The Netherlands) (33), in the first week of the first supplementation period. After a warming-up of five minutes at 75 Watt, the power was increased every 2.5 minutes by 50 Watt until $80 \%$ of the maximally calculated heart rate was reached, then the power was increased every 2.5 minutes by 25 Watt until exhaustion. The highest average oxygen consumption over 25 seconds was used as the $\mathrm{VO}_{2}$ peak, reflecting physical fitness of the participant.

\section{MR spectroscopy}

Proton magnetic resonance spectroscopy ( $\left.{ }^{1} \mathrm{H}-\mathrm{MRS}\right)$ was used to quantify intrahepatic lipid content (IHL), intramuscular lipid content (IMCL) and skeletal muscle acetylcarnitine concentrations. Phosphorus magnetic resonance spectroscopy $\left({ }^{31} \mathrm{P}\right.$ MRS) was used for assessment of phosphocreatine (PCr) to adenosine triphosphate (ATP) ratio as a marker of the energy status and in vivo mitochondrial function of the 
heart muscle. Magnetic resonance imaging was used for determination of cardiac left ventricle ejection fraction (EF). All measurements were performed on a 3.0T whole body scanner (Achieva Tx, Philips Healthcare, Best, The Netherlands).

IHL \& IMCL: IHL quantification took place on day 36 of each intervention at 17:00 $\mathrm{h}$. Participants were fasted for at least 3 hours. Spectra were acquired as described before (34). Values are given as T2 corrected ratios of the $\mathrm{CH}_{2}$ peak relative to the unsuppressed water peak, expressed as percentage. Consecutive, IMCL was measured in the $\mathrm{m}$. tibialis anterior of the left leg, as reported earlier (34). Values are given as T1- and T2-corrected ratios of the $\mathrm{CH}_{2}$ peak relative to the unsuppressed water peak, expressed as percentage. Due to analytical problems only 9 participants could be included in the analyses of IMCL.

Acetylcarnitine: Acetylcarnitine concentrations in skeletal muscle were acquired on day 32 of each intervention at 17:00 h in the evening. Participants were fasted for at least 3 hours and were asked to refrain from strenuous physical activity 48 hours prior to the measurement. Resting skeletal muscle Acetylcarnitine concentrations were measured using a T1-editing method, as described earlier (35). Additionally, Acetylcarnitine concentrations were measured after 30 minutes exercise $(70 \%$ maximal output on an ergometer). Acetylcarnitine values were converted to absolute concentrations as described earlier (36). The creatine peak was used as a reference.

Cardiac PCr/ATP ratio \& ejection fraction: PCr/ATP ratio was quantified by ${ }^{31} \mathrm{P}-\mathrm{MRS}$ on day 37 of each intervention at 06:30 h, using an ISIS sequence. Participants were positioned prone and headfirst in the MRI. A ${ }^{1} \mathrm{H}^{31} \mathrm{P}$ surface heart coil was placed beneath the participants chest. The voxel of interest was carefully placed around the left ventricle of the heart. Spectra were acquired during the end-systolic phase (NSA $=96$, number of points $=2048$, bandwidth $=3000 \mathrm{~Hz}$ ) with a repetition time of 5-8 heartbeats, depending on heart rate. $\mathrm{PCr}$ and ATP resonances were quantified using a custom written MATLAB (MATLAB 2014b, The MathWorks, Inc., Natick, Massachusetts, United States) script and values were corrected for T1 saturation and expressed as ratio of PCr over gamma-ATP. Due to technical errors, only 11 participants are included in the analyses of PCr/ATP ratio. Left ventricular size was measured for 12 participants based of MRI images of the heart. Left ventricular EF was calculated from the end diastiolic volume (EDV) and end systolic volume (ESV) according to the Biplane Ellipsoid Model, as described earlier (37). 


\section{Ambulatory blood pressure}

Ambulatory blood pressure (BP) was measured (Mobil-O-Graph, I.E.M., Stolberg, Germany) on day 38 and 39 of each intervention for two days and one night (36hours). Mean systolic (SBP) and diastolic (DBP) blood pressure during day time and night time, and night time dipping were calculated as previously described (38). Night was defined as the mean time going to bed of all participants (23:38 h) until the mean time waking up of all participants (8:05 h). Due to technical failures night time BP could only by obtained from 12 participants.

\section{Body composition}

Body composition was determined on day 40 of each intervention at 08:30 h after an overnight fast of at least 10 hours. Body mass and body volume were assessed using air-displacement plethysmography using the BodPod device (Cosmed, Italy, Rome) according to the manufacturer's instructions (39) and previously reported (40).

\section{Blood sampling and analyses}

Glucose (Hk-CP, Axonlab, Amsterdam, The Netherlands) and free fatty acids (FFA) (NEFA-HR, WAKO chemicals, Neuss, Germany) were analyzed enzymatically in EDTA plasma using a Pentra 400 (Horiba, Montpellier, France). Triglycerides (Sigma, Zwijndrecht, The Netherlands), cholesterol (CHOD-PAP, Roche Diagnostics, Mannheim, Germany) and HDL-C (CHOD-PAP, Roche Diagnostics, Mannheim,Germany) after precipitation of apoB-containing lipoproteins with phosphotungstic acid and magnesium ions, were analyzed in serum also using a Pentra 400. All samples from one participant were analyzed within one run. LDL-C was calculated for 12 participants according to the Friedewald equation (41). In a subset of 7 participants (age $60 \pm 3$ years; BMl $30.0 \pm 1.7 \mathrm{~kg} / \mathrm{m}^{2} ; \mathrm{n}=2$ women) inflammatory cytokine concentrations were measured on a Luminex® 200TM system using an inflammation 20-plex human Procartaplex panel (eBioscience, EPX20012185-901) containing markers for sE-Selectin; ICAM-1/CD54; IL-1 $\alpha$; IL-4; IL-12p70;

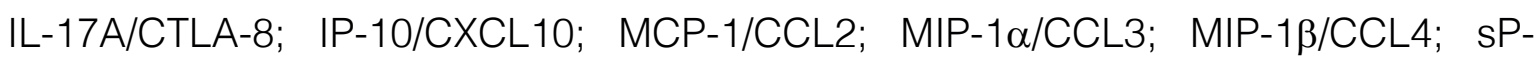
Selectin; TNF $\alpha$.

\section{Calculations}

Energy expenditure was calculated based on the measured averaged oxygen and carbon dioxide concentrations in the inspired and expired gasses with the assumption that protein oxidation was negligible, using the Weir equation $(42,43)$. Sleeping metabolic rate was calculated as the lowest average 3 h energy expenditure during the sleeping period. Glucose oxidation and fat oxidation rates were calculated 
according to Perronet et al. $(42,43)$. Steele's single pool non-steady state equations were used to calculate the rate of glucose appearance $(\mathrm{Ra})$ and the rate of glucose disappearance (Rd) during the clamp (44). Volume of distribution was assumed to be $0.160 \mathrm{l} / \mathrm{kg}$ for glucose. The change in insulin-stimulated glucose disposal (delta Rd) was calculated by the difference between Rd under insulin-stimulated conditions and $\mathrm{Rd}$ under basal non-insulin-stimulated conditions. Endogenous glucose production (EGP) was calculated as Ra minus exogenous glucose infusion rate. Non-oxidative glucose disposal (NOGD) was calculated as Rd minus carbohydrate oxidation.

\section{Sample size}

The sample size was determined based on demonstrating the statistical superiority of NR on insulin stimulated skeletal muscle glucose disposal compared with placebo. Twelve participants were required to achieve $80 \%$ power with an alpha of $5 \%$, an assumed treatment difference of within person changes of $3.25 \mu \mathrm{mol} / \mathrm{kg} / \mathrm{min}$, and an assumed SD of within person changes of $3.60 \mu \mathrm{mol} / \mathrm{kg} / \mathrm{min}$ for a one-group paired ttest for a hyperinsulinemic euglycemic clamp. A dropout of $20 \%$ was taken into account, so 15 participants were recruited. The expected effect size and SD was based on previous research within our research group (45).

\section{Statistical analyses}

Data are reported as mean \pm SE, unless otherwise stated. Data are presented for $n=13$, unless otherwise indicated. Differences between interventions were analyzed with a two-tailed paired Students t-test for parametric data and with a Wilcoxon test for non-parametric data. A two-tailed $p<0.05$ was considered statistically significant. Statistical analyses were performed using IBM SPSS version 23.0 for MacOS.

\section{RESULTS}

\section{Participant population and study compliance}

Thirteen healthy overweight and obese men and women (age $59 \pm 5$ years; BMI 30.2 $\pm 2.6 \mathrm{~kg} / \mathrm{m}^{2}$ [mean $\pm \mathrm{SD}$ ]; 7 women) participated in the study. Participants were nonsmokers, had no active diseases, used no medication or supplements interfering with the study outcomes, had a sedentary lifestyle according to the Baecke questionnaire $\left(7.51 \pm 1.16\right.$ arbitrary units [mean $\pm \mathrm{SD}$ ]) and an average $\mathrm{VO}_{2}$ peak of $27.0 \pm 5.7$ $\mathrm{ml} / \mathrm{min} / \mathrm{kg}$ [mean $\pm \mathrm{SD}$ ] (see Supplementary Table 2). NR at $1000 \mathrm{mg} /$ day was well tolerated and no adverse events or side effects were reported. Surplus NR and placebo supplements were returned by the participants and compliance rate was 
calculated as the proportion of capsules ingested relative to the prescribed number. The mean compliance rate during the six-week NR period was $99.3 \pm 1.8 \%$ [mean \pm $\mathrm{SD}$ ] and during the six-week placebo period was $99.1 \pm 2.2 \%$ [mean \pm SD]. Participants were instructed to maintain their habitual diet and physical activity pattern during the entire study, this was confirmed by a stable body weight of $85.2 \pm 3.8 \mathrm{~kg}$ [mean $\pm \mathrm{SD}$ ] after six weeks of NR supplementation compared to $85.5 \pm 3.8 \mathrm{~kg}$ [mean \pm SD] upon six weeks placebo $(p=0.55)$.

A

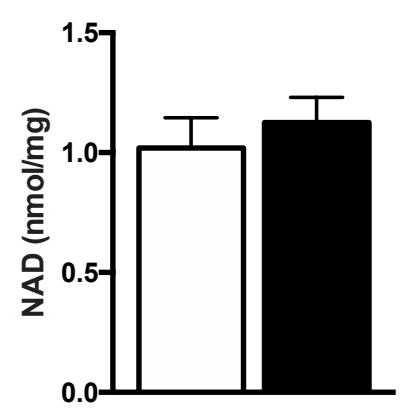

B

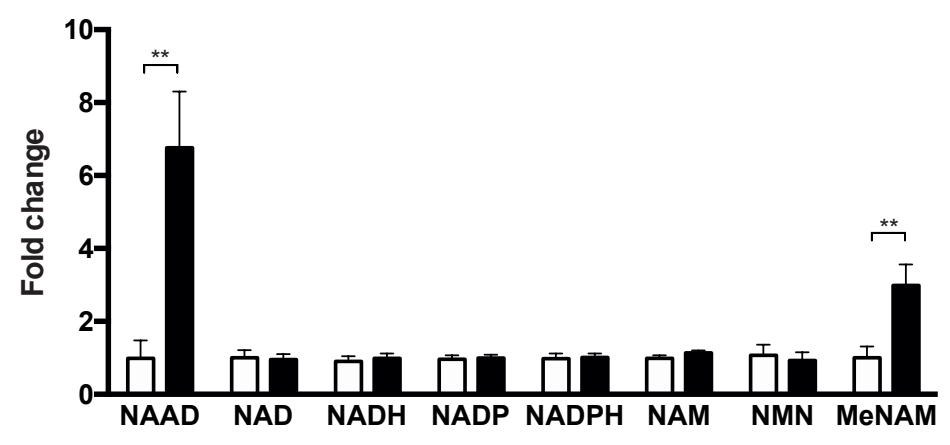

Figure 2. $\mathrm{NAD}^{+}$metabolites in skeletal muscle after $\mathrm{NR}$ and placebo supplementation

$A: N A D^{+}$concentrations measured in skeletal muscle biopsies by enzymatic assay, $n=8$. B: NAD+ metabolites measured in skeletal muscle biopsies by mass spectrometry, $n=12$. Black bars is NR, open bars is placebo. Data are expressed as mean \pm SE. P values are derived from the analysis of the mean within-person changes and the SEM of the within-group changes. ${ }^{\star *} \mathrm{p}<0.01$.

\section{$\mathrm{NAD}^{+}$metabolites in skeletal muscle}

Compliance was further checked by analysis of $\mathrm{NAD}^{+}$-derived metabolites in muscle biopsy samples collected after six weeks of NR and placebo, 14-16 hours after supplement intake. First skeletal muscle NAD+ content was measured. Quantitative analyses of $\mathrm{NAD}^{+}$concentrations using enzymatic cycling assay in skeletal muscle showed that NAD ${ }^{+}$content was not different between NR and placebo (1.019 \pm 0.126 vs. $1.125 \pm 0.106,0.106 \pm 0.105 \mathrm{nmol} / \mathrm{mg}$ dry weight in NR, placebo, and the change respectively, $p=0.34, n=8$, Figure $2 A$ ). The lack of increase in $\mathrm{NAD}^{+}$may indicate that $N A D^{+}$flux is increased without elevated steady state NAD+ concentrations. Therefore, we next performed metabolomics to check if $\mathrm{NAD}^{+}$and $\mathrm{NAD}^{+}$metabolites in skeletal muscle were affected by NR. We confirmed that NR supplementation had no effect on skeletal muscle $\mathrm{NAD}^{+}$content itself $(0.96 \pm 0.15$ fold change in NR compared to placebo, $p=0.91, n=12$, Figure $2 B$ ). However, oral NR supplementation significantly increased two main markers of enhanced NAD+ metabolism - nicotinic acid adenine dinucleotide (NAAD, $6.8 \pm 1.5$ fold change $p=<0.01, n=12$, Figure $2 B$ ) and 
methylnicotinamide (MeNAM, $3.0 \pm 0.6$ fold change $p=<0.01, n=12$, Figure $2 B$ ), confirming that indeed NR was amplifying skeletal muscle NAD+ metabolism without affecting steady state. Moreover, NADH, NADP, NADPH, NAM and NMN concentrations remained unchanged $(p=0.73, p=0.79, p=0.75, p=0.25$ and $p=0.97$ respectively, $n=12$, see Figure $2 B$ ).

\section{Mitochondrial respiration in skeletal muscle}

Supplementation with NR did not result in any change in mitochondrial respiration compared ot the placebo state. Respiration in the presence of substrate alone (state 2) (malate (M), malate + octanoyl carnitine (MO2) or malate + glutamate (MG2)) was not different between conditions $(p=0.34, p=0.19, p=0.74$ respectively, Figure $3 A)$. Furthermore, ADP stimulated (state 3 ) respiration on lipid-derived substrate (malate + octanoyl carnitine + ADP (MO3)) and upon complex I substrates (malate + glutamate + ADP (MG3)) was unchanged $(p=0.67$ and $p=0.64$ respectively, Figure 3B and $3 \mathrm{C}$ ). Respiration upon parallel electron input to both complex I and II (malate + octanoyl carnitine + glutamate (MOG3)) was not different between conditions (49.87 \pm 2.80 vs. $50.92 \pm 2.44 \mathrm{pmol} / \mathrm{mg} / \mathrm{s},-1.04 \pm 2.58 \mathrm{in} \mathrm{NR}$, placebo, and the change respectively, $p=0.69$, Figure $3 D$ ). Similar results were observed when succinate was sequentially added in both experiments (MGS3: $74.27 \pm 3.27$ vs. $72.90 \pm 4.64,1.37$ $\pm 5.01 \mathrm{pmol} / \mathrm{mg} / \mathrm{s}$ in NR, placebo, and the change respectively, $\mathrm{p}=0.79$; MOGS3: $75.30 \pm 3.92$ vs. $76.09 \pm 3.31,-0.79 \pm 4.36 \mathrm{pmol} / \mathrm{mg} / \mathrm{s}$ in NR, placebo, and the change respectively, $\mathrm{p}=0.86$, Figure $3 \mathrm{D}$ ). Maximal FCCP-induced uncoupled respiration (state $u$ ), reflecting the maximal capacity of the electron transport chain, was also unchanged ( $p=0.81$, Figure $3 E$ ). Finally, state 40 respiration (reflecting proton leak) was similar after NR and placebo conditions ( $p=0.89$, Figure 3F). For a description of the different states, please see: https://www.bioblast.at/index.php/ MitoPedia:_Respiratory_states. Mitochondrial OXPHOS protein concentrations (Complex I $0.96 \pm 0.21$ vs. $0.91 \pm 0.15,0.05 \pm 0.235$ A.U. in NR, placebo, and the change respectively, $p=0.84$; Complex $\| 1.01 \pm 0.17$ vs. $0.78 \pm 0.05,0.23 \pm 0.20$ A.U. in NR, placebo, and the change respectively, $p=0.64$; Complex III $0.94 \pm 0.19$ vs. $0.92 \pm 0.17,0.02 \pm 0.22$ A.U. in NR, placebo, and the change respectively, $p=0.93$ Complex IV $1.02 \pm 0.15$ vs. $0.94 \pm 0.10,0.09 \pm 0.14$ A.U. in NR, placebo, and the change respectively $p=0.55$; Complex V $1.00 \pm 0.17$ vs. $0.79 \pm 0.16,0.21 \pm 0.17 \mathrm{~A} . U$. in NR, placebo, and the change respectively $p=0.24)$ were similar after $N R$ and placebo supplementation, indicating no effect of NR on mitochondrial content (Figure $3 G$ ). 
A

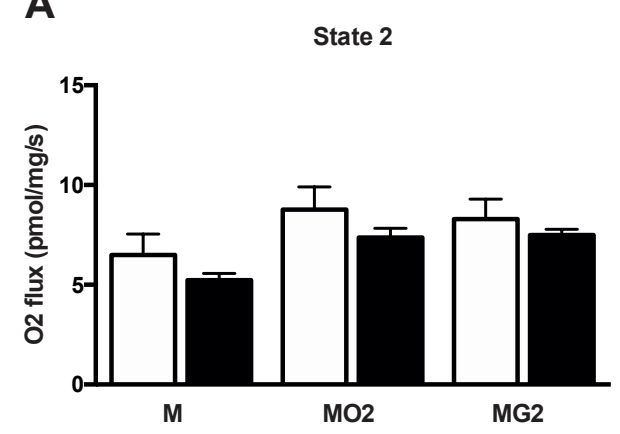

D

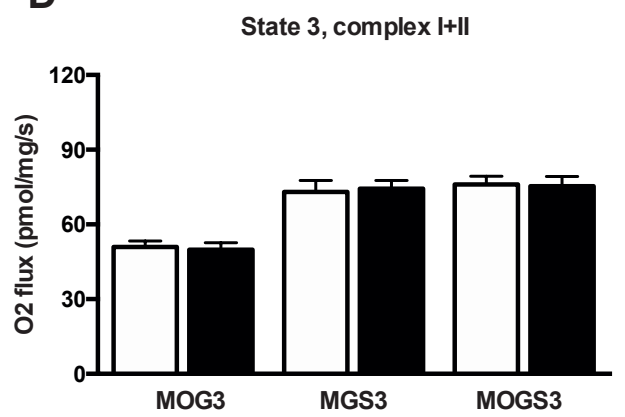

B State 3, fat oxidation

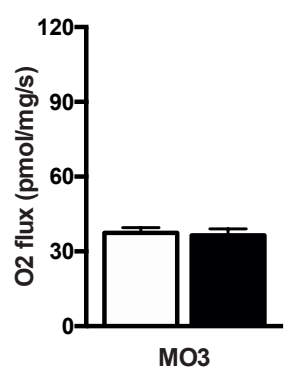

E

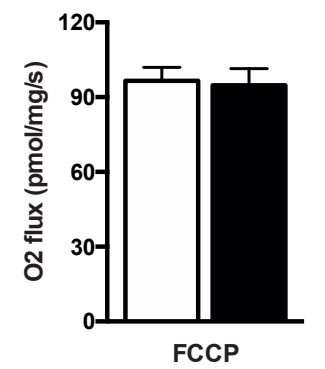

C State 3, complex I

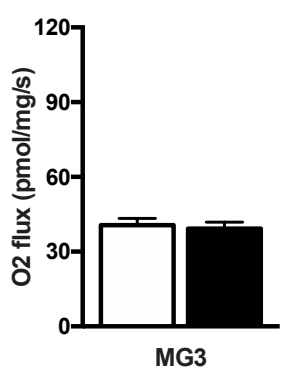

$\mathbf{F}$ State 4o, complex I+II

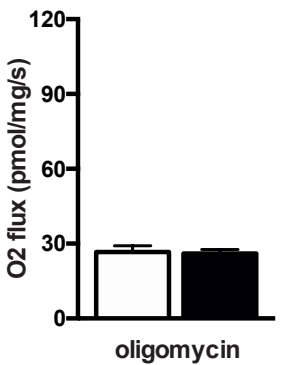

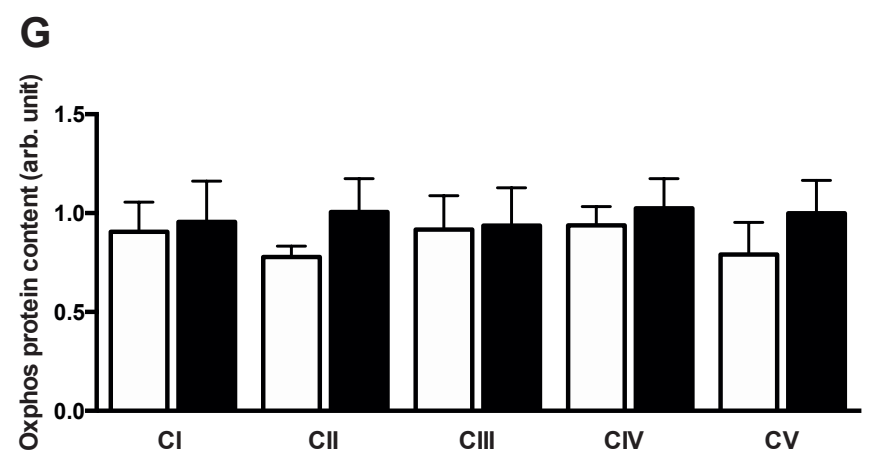

Figure 3. Skeletal muscle ex vivo mitochondrial respiratory capacity after NR and placebo supplementation

A: State 2 respiration upon malate $(\mathrm{M})$, malate + octanoyl carnitine $(\mathrm{MO} 2)$ and malate + glutamate (MG2). B: ADP stimulated state 3 respiration upon lipid derived substrate, malate + octanoyl carnitine + glutamate (MO3). C: ADP stimulated state 3 respiration upon complex I substrates, malate + glutamate (MG3). D: ADP stimulated state 3 respiration upon parallel electron input to both complex I and II, malate + octanoyl carnitine + glutamate (MOG3), malate + glutamate + succinate (MGS3), malate + octanoyl carnitine + glutamate + succinate (MOGS3). E: State u: maximal FCCP-induced uncoupled respiration. F: State 40: Oligomycin induced respiration not coupled to ATP synthesis. G: Protein content of individual complexes of the electron transport chain. Black bars is $N R$, open bars is placebo. $N=12$. Data are expressed as mean $\pm \mathrm{SE}$. 


\section{Acetylcarnitine concentrations in skeletal muscle}

Quantification of acetylcarnitine concentration in skeletal muscle, measured by MRS technique in the early evening at 17:00 h after 3 hours fasting, revealed significantly lower baseline acetylcarnitine concentrations under NR supplementation compared to placebo $(1.30 \pm 0.16$ vs. $1.80 \pm 0.18,-0.53 \pm 0.19 \mathrm{mmol} / \mathrm{kg}$ wet weight in $\mathrm{NR}$, placebo, and the change respectively, $p=0.02$, Figure 4A). Maximally stimulated acetylcarnitine concentrations, measured upon exercise, were not different between conditions $(4.29 \pm 0.29$ vs. $4.20 \pm 0.27,0.00 \pm 0.20 \mathrm{mmol} / \mathrm{kg}$ wet weight in $\mathrm{NR}$, placebo, and the change respectively, $p=0.67$, Figure 4A). Nonetheless, the capacity to increase acetylcarnitine formation, expressed as the change computed as the post exercise value minus the baseline value, was significantly higher in NR compared to placebo ( $2.99 \pm 0.30$ vs. $2.40 \pm 0.33,0.53 \pm 0.21 \mathrm{mmol} / \mathrm{kg}$ wet weight in NR, placebo, and the change respectively, $p=0.01$, Figure $4 \mathrm{~A}$ ). Based on these results, we decided to perform full acylcarnitine analysis in skeletal muscle biopsies taken at 8:30 h after an overnight fast. Remarkably, acetylcarnitine (C2) concentrations were significantly higher under NR supplementation compared to placebo (C2: $4558 \pm 749$ vs. $3025 \pm$ $316,1533 \pm 683 \mathrm{pmol} / \mathrm{mg}$ dry weight in NR, placebo, and the change respectively, $\mathrm{p}=0.04$; Figure 4B). No differences were detected in free carnitine (C0), other short chain (C3 to C5), medium chain (C6 to C12) or long chain acylcarnitines (C13 to C20) between NR and placebo $(p=0.25, p=0.27, p=0.99$ and $p=0.45$ respectively; Figure 4C).

\section{Insulin sensitivity and substrate kinetics}

Potential effects of NR on whole body and tissue-specific insulin sensitivity were assessed by a two-step hyperinsulinemic-euglycemic clamp. Whole body insulin stimulated glucose uptake, as expressed by the change in glucose disposal (delta $\mathrm{Rd}$ ) from baseline to high insulin dose, was not different between NR and placebo $(p=0.98$, Table 1$)$. Hepatic insulin sensitivity, reflected by EGP suppression (EGP\%) during the low insulin phase, was not affected by NR compared to placebo $(p=0.30$, Table 1). NR had no effect on baseline substrate oxidation (carbohydrate oxidation $p=0.84$, and fat oxidation $p=0.67$ ). Furthermore, insulin stimulated carbohydrate oxidation or suppression of fatty acid oxidation, reflecting metabolic flexibility, was not different between NR and placebo ( $p=0.92$ and $p=0.70)$ (see Table 1). In addition, non-oxidative glucose disposal (delta NOGD) during low and high insulin phase of the clamp remained unchanged between conditions $(p=0.88$ and $p=0.99)$ (see Table 1). Plasma FFA concentrations were suppressed by insulin to a similar extend between NR and placebo ( $p=0.97$ and $p=0.99$ during low and high insulin phase respectively, Table 1 ), indicating similar white adipose tissue insulin sensitivity. 
A

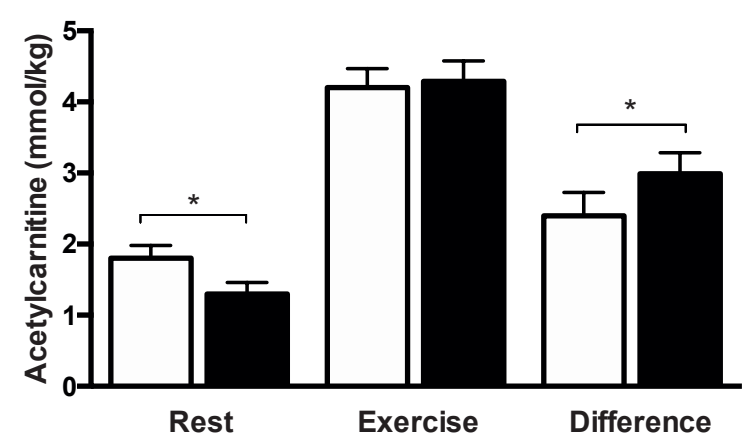

C

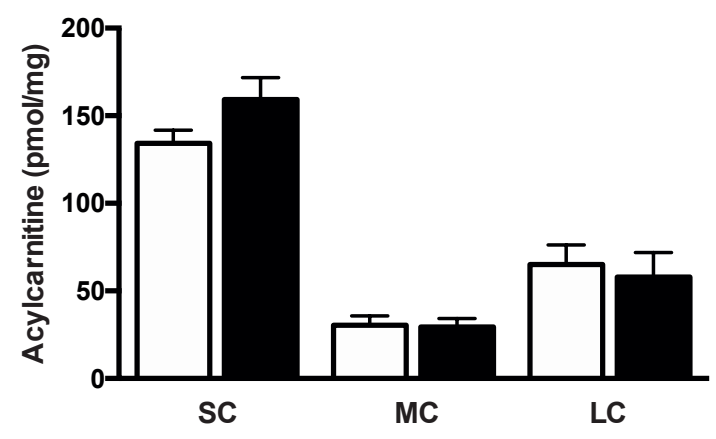

B

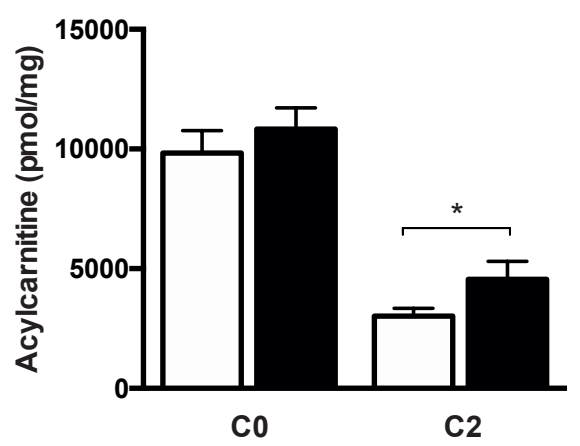

Figure 4. Skeletal muscle acylcarnitine concentrations measured in the morning and evening after NR and placebo supplementation

A: Acetylcarnitine concentration measured by MRS in skeletal muscle in the evening during rest, after exercise and the capacity to form acetylcarnitine expressed as the difference between rest and exercise. B: Free carnitine (C0) and acetylcarnitine (C2) concentrations measured in muscle biopsies in the morning during rest. C: Sum of short chain acylcarnitines (SC), sum of medium chain acylcarnitines (MC) and sum of long chain acylcarnitines (LC) concentrations measured in biopsies during rest in the morning. Black bars is $N R$, open bars is placebo. $N=13$. Data are expressed as mean \pm SE. $P$ values are derived from the analysis of the mean within-person changes and the SEM of the within-group changes. ${ }^{*} p<0.05$.

\section{Plasma biochemistry and inflammatory markers}

NR supplementation did not affect fasting plasma glucose, triglycerides, total cholesterol, HDL-C, LDL-C, or inflammatory markers, including chemokine, cytokine or cell-adhesion molecule concentrations (Table 2). However, interleukin 1 alpha (IL$1 \alpha)$ tended to be lower after NR supplementation compared to placebo $(1.61 \pm 0.28$ vs. $2.11 \pm 0.35$ respectively, $p=0.06$, Table 2 ). 
Table 1. Insulin sensitivity and substrate kinetics

\begin{tabular}{|c|c|c|c|c|}
\hline Parameter & Placebo & NR & Change & $p$-value \\
\hline \multicolumn{5}{|l|}{$\mathrm{Ra}(\mu \mathrm{mol} / \mathrm{kg} / \mathrm{min})^{1}$} \\
\hline Baseline & $9.32 \pm 0.33$ & $9.54 \pm 0.78$ & $0.22 \pm 0.72$ & 0.77 \\
\hline Low insulin & $11.66 \pm 0.93$ & $11.74 \pm 0.91$ & $0.08 \pm 0.42$ & 0.85 \\
\hline High insulin & $36.70 \pm 3.40$ & $37.60 \pm 2.66$ & $0.91 \pm 2.24$ & 0.69 \\
\hline \multicolumn{5}{|l|}{$\operatorname{Rd}(\mu \mathrm{mol} / \mathrm{kg} / \mathrm{min})^{1}$} \\
\hline Baseline & $9.90 \pm 0.53$ & $9.52 \pm 0.84$ & $-0.38 \pm 0.97$ & 0.70 \\
\hline Low insulin & $12.06 \pm 1.00$ & $12.36 \pm 0.94$ & $0.30 \pm 0.45$ & 0.51 \\
\hline High insulin & $36.76 \pm 3.36$ & $36.32 \pm 2.62$ & $-0.45 \pm 2.13$ & 0.84 \\
\hline Delta baseline-low insulin & $2.16 \pm 1.07$ & $2.84 \pm 1.01$ & $0.68 \pm 1.03$ & 0.47 \\
\hline Delta baseline-high insulin & $26.86 \pm 3.31$ & $26.79 \pm 2.86$ & $-0.07 \pm 2.17$ & 0.98 \\
\hline \multicolumn{5}{|l|}{$\mathrm{EGP}(\mu \mathrm{mol} / \mathrm{kg} / \mathrm{min})^{1}$} \\
\hline Baseline & $9.32 \pm 0.33$ & $9.54 \pm 0.78$ & $0.22 \pm 0.72$ & 0.77 \\
\hline Low insulin & $2.70 \pm 0.40$ & $3.38 \pm 0.56$ & $0.68 \pm 0.48$ & 0.18 \\
\hline$\%$ suppression low insulin & $70.44 \pm 4.57$ & $61.02 \pm 7.05$ & $-9.42 \pm 7.13$ & 0.30 \\
\hline High insulin & $-0.04 \pm 0.26$ & $0.51 \pm 0.56$ & $0.55 \pm 0.51$ & 0.30 \\
\hline$\%$ suppression high insulin & $99.78 \pm 2.52$ & $96.82 \pm 4.65$ & $-2.95 \pm 4.28$ & 0.50 \\
\hline \multicolumn{5}{|l|}{$\operatorname{NOGD}(\mu \mathrm{mol} / \mathrm{kg} / \mathrm{min})^{1}$} \\
\hline Baseline & $5.27 \pm 1.22$ & $4.88 \pm 1.06$ & $-0.40 \pm 1.18$ & 0.74 \\
\hline Low insulin & $3.77 \pm 0.92$ & $3.56 \pm 0.75$ & $-0.19 \pm 0.63$ & 0.76 \\
\hline High insulin & $21.28 \pm 2.84$ & $20.85 \pm 2.01$ & $-0.43 \pm 2.03$ & 0.84 \\
\hline Delta baseline-low insulin & $-1.51 \pm 1.36$ & $-1.30 \pm 0.85$ & $0.21 \pm 1.34$ & 0.88 \\
\hline Delta baseline-high insulin & $16.01 \pm 2.81$ & $15.97 \pm 2.04$ & $-0.03 \pm 1.83$ & 0.99 \\
\hline \multicolumn{5}{|c|}{ Carbohydrate oxidation ( $\mu \mathrm{mol} / \mathrm{kg} / \mathrm{min})$} \\
\hline Baseline & $4.42 \pm 0.81$ & $4.58 \pm 0.55$ & $0.16 \pm 0.61$ & 0.84 \\
\hline Low insulin & $7.84 \pm 0.69$ & $8.79 \pm 0.78$ & $0.95 \pm 0.76$ & 0.23 \\
\hline High insulin & $14.90 \pm 0.96$ & $14.99 \pm 1.15$ & $0.09 \pm 0.89$ & 0.92 \\
\hline \multicolumn{5}{|l|}{ Fat oxidation ( $\mu \mathrm{mol} / \mathrm{kg} / \mathrm{min})$} \\
\hline Baseline & $3.78 \pm 0.23$ & $3.71 \pm 0.20$ & $-0.07 \pm 0.17$ & 0.67 \\
\hline Low insulin & $2.73 \pm 0.19$ & $2.55 \pm 0.17$ & $-0.18 \pm 0.21$ & 0.41 \\
\hline High insulin & $1.47 \pm 0.23$ & $1.38 \pm 0.22$ & $-0.09 \pm 0.22$ & 0.70 \\
\hline \multicolumn{5}{|l|}{ Plasma FFA ( $\mu \mathrm{mol} / \mathrm{l})$} \\
\hline Baseline & $555.34 \pm 30.87$ & $581.16 \pm 32.08$ & $25.83 \pm 35.55$ & 0.48 \\
\hline Low insulin & $128.55 \pm 21.74$ & $128.03 \pm 19.26$ & $-0.52 \pm 14.19$ & 0.97 \\
\hline High insulin & $49.84 \pm 8.26$ & $56.45 \pm 14.80$ & $6.61 \pm 10.04$ & 0.99 \\
\hline \multicolumn{5}{|l|}{ Respiratory exchange ratio } \\
\hline Baseline & $0.77 \pm 0.01$ & $0.77 \pm 0.01$ & $0.00 \pm 0.01$ & 0.83 \\
\hline Low insulin & $0.82 \pm 0.01$ & $0.83 \pm 0.01$ & $0.01 \pm 0.01$ & 0.37 \\
\hline High insulin & $0.91 \pm 0.01$ & $0.91 \pm 0.01$ & $0.00 \pm 0.01$ & 0.86 \\
\hline
\end{tabular}

Ra, rate of appearance; $R d$, rate of disappearance; EGP, endogenous glucose production; NOGD, non-oxidative glucose disposal; FFA, free fatty acids. ${ }^{n} n=12$. Data are expressed as mean \pm SE. P values are derived from the analysis of the mean within-person changes and the SE of the within-group changes. 
Table 2. Blood biochemistry

\begin{tabular}{lllll}
\hline Parameter & Placebo & NR & Change & p-value \\
\hline Glucose $(\mathrm{mmol} / \mathrm{l})$ & $5.48 \pm 0.14$ & $5.44 \pm 0.13$ & $-0.04 \pm 0.10$ & 0.70 \\
Triglycerides $(\mathrm{mmol} / \mathrm{l})$ & $1.57 \pm 0.35$ & $1.63 \pm 0.38$ & $0.06 \pm 0.08$ & 0.24 \\
Total cholesterol $(\mathrm{mmol} / \mathrm{l})$ & $5.54 \pm 0.30$ & $5.55 \pm 0.35$ & $0.01 \pm 0.11$ & 0.99 \\
HDL-C $(\mathrm{mmol} / \mathrm{l})$ & $1.32 \pm 0.12$ & $1.32 \pm 0.09$ & $-0.00 \pm 0.04$ & 0.99 \\
LDL-C $(\mathrm{mmol} /)^{1}$ & $3.42 \pm 0.18$ & $3.37 \pm 0.17$ & $-0.05 \pm 0.07$ & 0.52 \\
sE-selectin $(\mathrm{pg} / \mathrm{ml})^{2}$ & $32726 \pm 5298$ & $34765 \pm 4354$ & $686 \pm 2068$ & 0.99 \\
sP-selectin $(\mathrm{pg} / \mathrm{ml})^{2}$ & $21428 \pm 3662$ & $26645 \pm 4065$ & $2624 \pm 3739$ & 0.58 \\
ICAM-1 $(\mathrm{pg} / \mathrm{ml})^{2}$ & $92493 \pm 19326$ & $129236 \pm 34547$ & $35350 \pm 27518$ & 0.58 \\
TNF- $\alpha(\mathrm{pg} / \mathrm{ml})^{2}$ & $29.80 \pm 6.38$ & $31.85 \pm 6.14$ & $2.70 \pm 1.97$ & 0.22 \\
IL-1 $\alpha(\mathrm{pg} / \mathrm{ml})^{2}$ & $2.11 \pm 0.35$ & $1.61 \pm 0.28$ & $-0.57 \pm 0.24$ & 0.06 \\
IL-4 $(\mathrm{pg} / \mathrm{ml})^{2}$ & $8.04 \pm 1.03$ & $8.86 \pm 1.41$ & $0.71 \pm 3.63$ & 0.69 \\
IL-12p70 $(\mathrm{pg} / \mathrm{ml})^{2}$ & $76.07 \pm 2.73$ & $78.89 \pm 3.02$ & $1.94 \pm 2.82$ & 0.81 \\
IL-17 $\alpha(\mathrm{pg} / \mathrm{ml})^{2}$ & $6.36 \pm 1.36$ & $7.95 \pm 2.09$ & $1.76 \pm 1.02$ & 0.16 \\
CXCL10 $(\mathrm{pg} / \mathrm{ml})^{2}$ & $3.71 \pm 0.55$ & $3.39 \pm 0.50$ & $-0.61 \pm 0.40$ & 0.22 \\
CCL2 $(\mathrm{pg} / \mathrm{ml})^{2}$ & $127.39 \pm 24.54$ & $144.34 \pm 47.86$ & $21.42 \pm 40.12$ & 0.69 \\
CCL3 $(\mathrm{pg} / \mathrm{ml})^{2}$ & $15.42 \pm 8.59$ & $17.04 \pm 10.39$ & $2.43 \pm 1.92$ & 0.30 \\
CCL4 $(\mathrm{pg} / \mathrm{ml})^{2}$ & $23.89 \pm 5.98$ & $28.49 \pm 7.51$ & $4.52 \pm 4.45$ & 0.38
\end{tabular}

Blood samples were taken in week six of NR supplementation and placebo after an overnight fast. ${ }^{1} n=12,{ }^{2} n=7$. Data are expressed as mean \pm SE. $P$ values are derived from the analysis of the mean within-person changes and the SE of the within-group changes.

\section{Body composition}

After six weeks of NR and placebo supplementation, several changes in body composition were detected. Percentage fat free mass (FFM) was significantly higher after NR compared to placebo (62.65 \pm 2.49 vs. $61.32 \pm 2.58,1.34 \pm 0.50 \%$ in NR, placebo, and the change respectively, $p=0.02$, Figure $5 \mathrm{~B}$ ). In line with this, percentage fat mass (FM) was significantly lower after NR compared to placebo (37.35 \pm 2.49 vs. $38.68 \pm 2.58,-1.34 \pm 0.50 \%$ in NR, placebo, and the change respectively, $p=0.02$, Figure $5 \mathrm{~B})$. However, total bodyweight remained unchanged $(p=0.55$, Figure 5A).

\section{Sleeping metabolic rate}

Sleeping metabolic rate (SMR), measured during an overnight stay in a respiration chamber, was higher upon six weeks of NR compared to placebo (6.68 $\pm 0.30 \mathrm{vs}$. $6.49 \pm 0.31,0.19 \pm 0.08 \mathrm{MJ} /$ day in $\mathrm{NR}$, placebo, and the change respectively, $p=0.05$, Figure $5 \mathrm{C}$ ). This could be explained by the increase in FFM and sleeping metabolic rate per FFM was not significantly different $(0.127 \pm 0.003$ vs. $0.125 \pm 0.003,0.00 \pm$ $0.00 \mathrm{MJ} / F F M /$ day in NR, placebo, and the change respectively, $\mathrm{p}=0.48$, Figure $5 \mathrm{E}$ ). 
A

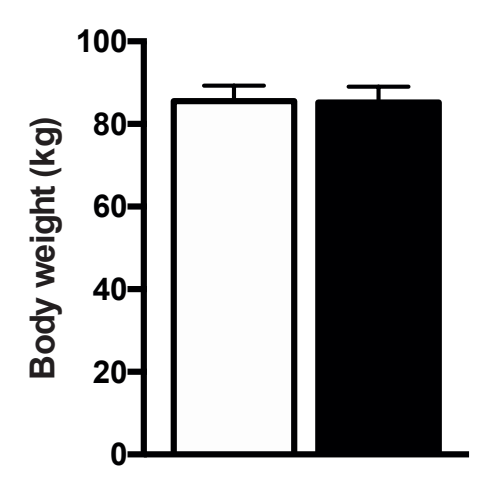

C

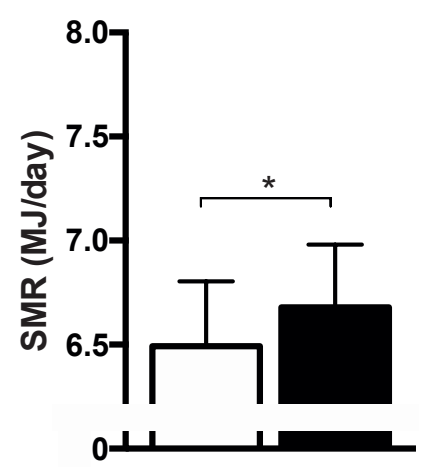

B
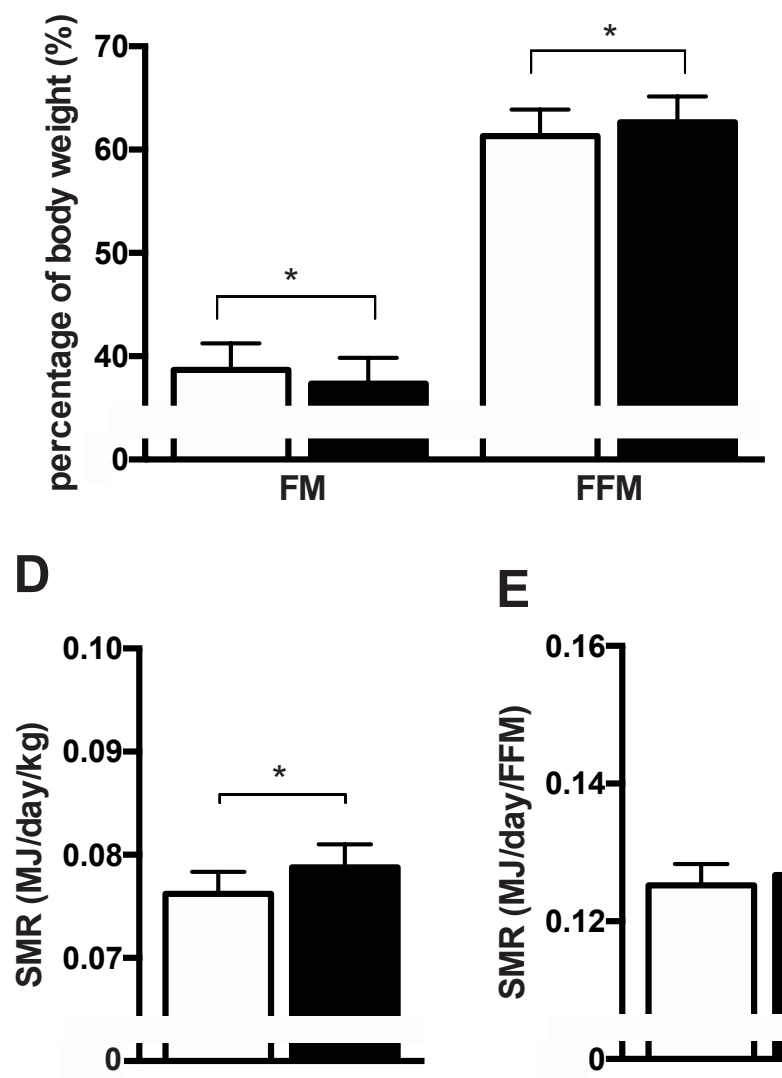

E

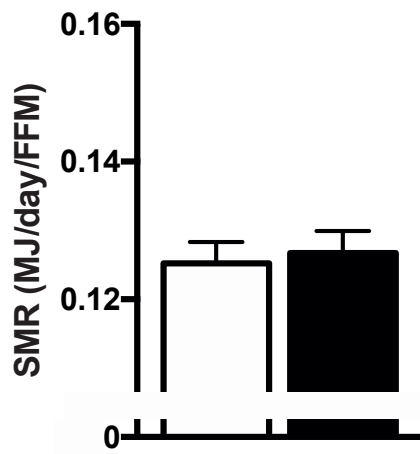

Figure 5. Body composition and sleeping metabolic rate after NR and placebo supplementation A: Bodyweight, fat mass (FM) and fat free mass (FFM) expressed in kilograms ( $\mathrm{kg}$ ). B: FM and FFM expressed as percentage (\%) of body weight. C: Sleeping metabolic rate expressed (SMR) as MJ per day. D: SMR corrected for body weight. E: SMR corrected for FFM. Black bars is NR, open bars is placebo. Data are expressed as mean $\pm S E$. $N=13$. $P$ values are derived from the analysis of the mean within-person changes and the SEM of the within-group changes. ${ }^{*} p<0.05$.

\section{Ectopic lipid storage}

Intrahepatic lipid content, measured by MRS, was not different between NR and placebo conditions $(3.4 \pm 1.2$ vs. $3.4 \pm 1.3,0.0 \pm 0.0 \%$ in NR, placebo, and the change respectively, $\mathrm{p}=0.85$ ). Furthermore, intramyocellular lipid content, measured by MRS technique in the $\mathrm{m}$. tibialis anterior, was not affected by NR supplementation $(0.5 \pm 0.1$ vs. $0.5 \pm 0.1,0.0 \pm 0.1 \%$ in NR, placebo, and the change respectively, $\mathrm{p}=0.50)$.

\section{Cardiac function}

To investigate if NR supplementation could affect cardiac energetics, we determined cardiac PCr/ATP ratios, which however were not affected by NR ( $p=0.90$, Table 3 ). No differences were observed in left ventricular end systolic volume (ESV), end 
diastolic volume (EDV), stroke volume (SV) and subsequently ejection fraction (EF) between NR and placebo $(p=0.23, p=0.72, p=0.69$ and $p=0.24$ respectively, Table 3). NR supplementation had no effect on 24-hour systolic blood pressure (SBP), diastolic blood pressure (DBP), mean arterial pressure (MAP), pulse pressure (PP), and heart rate $(H R)(p=0.56, p=0.39, p=0.40, p=0.60$ and $p=0.60$ respectively, Table 3). Separate analyses of day time and night time measurements did not reveal an effect of NR supplementation (see Table 3). In addition, night time dipping of SBP and DBP was not affected by NR compared to placebo $(p=0.53$ and $p=0.26$ respectively,

Table 3).

Table 3. Cardiometabolic health parameters

\begin{tabular}{|c|c|c|c|c|}
\hline Parameter & Placebo & NR & Change & $p$-value \\
\hline \multicolumn{5}{|l|}{ MRS Cardiac left ventricle ${ }^{2}$} \\
\hline Cardiac PCr/ATP ratio & $1.29 \pm 0.11$ & $1.22 \pm 0.09$ & $-0.08 \pm 0.14$ & 0.90 \\
\hline \multicolumn{5}{|l|}{ MRI Cardiac left ventricle ${ }^{1}$} \\
\hline EF (\%) & $71.2 \pm 2.2$ & $68.2 \pm 2.1$ & $-3.0 \pm 2.4$ & 0.24 \\
\hline ESV (ml) & $35.0 \pm 4.2$ & $38.9 \pm 3.9$ & $4.0 \pm 3.6$ & 0.23 \\
\hline EDV (ml) & $119.4 \pm 8.2$ & $121.2 \pm 8.3$ & $1.8 \pm 5.1$ & 0.72 \\
\hline SV (ml) & $84.4 \pm 5.6$ & $82.3 \pm 5.6$ & $-2.1 \pm 3.8$ & 0.69 \\
\hline \multicolumn{5}{|l|}{ Ambulatory BP 36-hour ${ }^{1}$} \\
\hline $\mathrm{SBP}(\mathrm{mmHg})$ & $124.6 \pm 2.4$ & $126.6 \pm 3.2$ & $2.0 \pm 2.3$ & 0.56 \\
\hline $\mathrm{DBP}(\mathrm{mmHg})$ & $77.1 \pm 1.8$ & $77.9 \pm 2.0$ & $0.8 \pm 0.9$ & 0.39 \\
\hline MAP (mmHg) & $98.9 \pm 1.7$ & $100.2 \pm 2.1$ & $1.3 \pm 1.5$ & 0.40 \\
\hline $\mathrm{PP}(\mathrm{mmHg})$ & $47.5 \pm 2.6$ & $48.6 \pm 3.1$ & $1.2 \pm 2.2$ & 0.60 \\
\hline HR (bpm) & $76.4 \pm 3.3$ & $75.5 \pm 3.6$ & $-0.9 \pm 1.7$ & 0.60 \\
\hline Night time dipping SBP (\%) & $10.6 \pm 2.4$ & $12.4 \pm 0.8$ & $1.8 \pm 2.8$ & 0.53 \\
\hline Night time dipping DBP (\%) & $11.9 \pm 2.4$ & $14.8 \pm 1.9$ & $3.0 \pm 2.5$ & 0.26 \\
\hline \multicolumn{5}{|l|}{ Ambulatory BP daytime } \\
\hline SBP $(\mathrm{mmHg})$ & $126.9 \pm 2.3$ & $129.0 \pm 2.9$ & $2.1 \pm 2.3$ & 0.54 \\
\hline $\mathrm{DBP}(\mathrm{mmHg})$ & $79.5 \pm 1.7$ & $80.5 \pm 1.9$ & $1.0 \pm 1.0$ & 0.32 \\
\hline MAP (mmHg) & $101.4 \pm 1.5$ & $102.7 \pm 1.9$ & $1.3 \pm 1.4$ & 0.39 \\
\hline $\mathrm{PP}(\mathrm{mmHg})$ & $47.6 \pm 2.7$ & $48.5 \pm 3.0$ & $0.9 \pm 2.1$ & 0.68 \\
\hline HR (bpm) & $78.3 \pm 3.2$ & $77.5 \pm 3.5$ & $-0.8 \pm 1.7$ & 0.67 \\
\hline \multicolumn{5}{|l|}{ Ambulatory BP night-time ${ }^{1}$} \\
\hline $\mathrm{SBP}(\mathrm{mmHg})$ & $113.5 \pm 3.4$ & $113.0 \pm 3.1$ & $-0.5 \pm 3.7$ & 0.90 \\
\hline $\mathrm{DBP}(\mathrm{mmHg})$ & $69.4 \pm 2.6$ & $68.1 \pm 2.3$ & $-1.3 \pm 1.5$ & 0.41 \\
\hline MAP (mmHg) & $89.6 \pm 2.7$ & $88.8 \pm 2.4$ & $-0.8 \pm 2.5$ & 0.75 \\
\hline $\mathrm{PP}(\mathrm{mmHg})$ & $43.8 \pm 2.5$ & $45.2 \pm 2.6$ & $1.4 \pm 2.3$ & 0.57 \\
\hline HR (bpm) & $65.6 \pm 2.5$ & $66.6 \pm 2.9$ & $1.0 \pm 1.9$ & 0.60 \\
\hline
\end{tabular}

PCr, phosphocreatin; ATP, adenosine triphosphate; EF, ejection fraction; ESV, end systolic volume; EDV, end diastolic volume; SV, stroke volume; BP, blood pressure; SBP, systolic blood pressure; DBP, diastolic blood pressure; MAP, mean arterial pressure; PP, pulse pressure; HR, heart rate. ${ }^{1} n=12$, ${ }^{2} n=11$. Data are expressed as mean \pm SE. $P$ values are derived from the analysis of the mean withinperson changes and the SE of the within-group changes. 


\section{DISCUSSION}

We hypothesized that NR supplementation in humans would increase NAD+ availability and thereby would improve a broad range of metabolic health parameters, mainly via improving mitochondrial function. To investigate this hypothesis, we performed a randomized double-blinded placebo controlled cross-over study with detailed metabolic phenotyping in which we provided healthy overweight and obese men and women 1000 mg/day NR for six weeks. In line with our hypothesis, NR supplementation did significantly increase markers of $\mathrm{NAD}^{+}$metabolism - NAAD and MeNAM - in skeletal muscle. This effect was accompanied by small but statistically significant improvements in body composition, sleeping metabolic rate and skeletal muscle acetylcarnitine concentrations, and a trend towards increased circulatory IL$1 \alpha$ concentrations. No further effects on skeletal muscle mitochondrial function, hepatic and whole-body insulin sensitivity, substrate oxidation, cardiovascular health markers and ectopic lipid accumulation were observed. However, it should be noted that many outcomes have been tested in our study and no adjustments for multiple comparisons were performed, therefore the possibility of false positive findings cannot be excluded. These results suggest that NR, at the dose of $1000 \mathrm{mg} /$ day for six weeks, did have, albeit relatively small, effects on metabolic parameters in humans, but was not effective in boosting muscle mitochondrial function or insulin sensitivity.

Animal studies showed that $\mathrm{NR}$ is able to increase plasma and tissue $\mathrm{NAD}^{+}$ concentrations (9-14). Also in humans, NR is able to increase circulatory NAD+ metabolites after several dosages ranging from $100 \mathrm{mg} /$ day to $2000 \mathrm{mg} /$ day (18-21). Here, we used a dose of $1000 \mathrm{mg} /$ day for six weeks, and in line with other data presented we did not report side effects $(18,20)$. We investigated if NR supplementation was able to increase the NAD+ metabolome in skeletal muscle tissue. In agreement with findings by Elhassan et al. (20) and Dollerup et al. (24), we show that NR supplementation increased $\mathrm{NAD}^{+}$metabolites NAAD and MeNAM in skeletal muscle, but without an increase in total $\mathrm{NAD}^{+}$content itself. NAAD is a highly sensitive biomarker of NR supplementation and increased NAD+ synthesis rate in tissues (14). MeNAM is part of the NAD+ degradation pathway and is a marker for increased NAD+ flux. These results might suggest that NR increases $N D^{+}$turnover rate, without affecting steady state $\mathrm{NAD}^{+}$concentrations in skeletal muscle.

We hypothesized that a NR-stimulated increase in NAD+ metabolism would lead to an increase in muscle mitochondrial function and a subsequent increase in human insulin sensitivity. However, in contrast to our hypothesis, skeletal muscle 
mitochondrial function was not elevated upon NR supplementation. This is in agreement with the findings of Elhassan et al. (25) and Dollerup et al. (24), who also reported no effect of NR on skeletal muscle mitochondrial function. Consistent with the lack of effect on mitochondrial function, we and others (21) did not observe improvements on insulin sensitivity upon NR supplementation. Although the limited duration of our and other studies may explain the lack of effect of NR on insulin sensitivity, we have previously shown that nutritional supplements like resveratrol can increase skeletal muscle mitochondrial function after four weeks of supplementation (45-47). Trammel et al. (48) and Dollerup et al. (21) suggested that the underlying pathway of metabolic improvements observed in obese mice was a decrease in hepatic lipid accumulation. In addition, Dollerup et al. (21) described a decrease in hepatic lipid content in obese men with elevated baseline hepatic lipid content, although this was not significant. Here, we did not observe an effect of NR on hepatic lipid accumulation, which may be contributed to our study population which had in general a healthy hepatic lipid content (i.e. $<5 \%$ liver fat).

NR supplementation has also been suggested to improve cardiovascular health (20, 49). Therefore, we here examined the effect of NR on cardiovascular health via detailed cardiovascular phenotyping. In contrast to Martens et al. (20) but in accordance with Conze et al. (23), we did not observe an effect of NR on blood pressure values. Martens et al. observed a decrease in resting SBP and DBP after NR, whereas we measured 36-hour ambulatory blood pressure, which gives a better estimation of blood pressure values and gives a better indication of the risk of cardiovascular events $(50,51)$. Consistent with the lack of effect of NR on blood pressure we did not find effects of NR on cardiac energy status or cardiac ejection fraction. Of note, the cardiac status of our participants was considered 'healthy', and in rodents also no change in cardiac function after NR supplementation in control mice with a healthy cardiac function could be observed (49).

We reported an improvement in body composition by an increase in percentage fat free mass mirrored by a decrease in percentage fat mass, while body weight remained unchanged. An effect of NR on body composition in humans has not been reported before $(20,21)$. Interestingly, in six out of seven women NR did increase fat free mass and reduce fat mass whereas this was only the case in one out of six men, suggesting that there might be a gender difference in the effect of NR supplementation on body composition. Consistent with the effect of NR on body composition, we show that the sleeping metabolic rate was also affected by NR supplementation and a higher metabolic rate could potentially lead to a reduction in fat mass. The increase in sleeping metabolic rate was due to an increase in fat free 
mass, suggesting that the primary effect of these findings could be an effect of NR on fat free mass. Interestingly, it has previously been shown that NAD+ metabolism and homeostasis is involved in maintaining muscle mass (13). Future studies should be designed to investigate if NR supplementation can indeed increase muscle mass in humans and investigate the underlying mechanisms.

Next to an effect of NR on muscle mass, NR supplementation enhanced the exerciseinduced increase in acetylcarnitine. Moreover, acetylcarnitine concentrations measured in skeletal muscle biopsies obtained in the morning, showed significantly increased concentrations after NR supplementation. These data suggest that NR is able to increase skeletal muscle acetylcarnitine metabolism, which has been associated with metabolic flexibility and improved metabolic health (36). Remarkably, though, resting acetylcarnitine concentrations measured using MRS three hours after lunch and before the exercise session were significantly lower after NR compared to placebo. It has previously been shown that meal consumption lowers acetylcarnitines in skeletal muscle $(52,53)$, but why NR would substantiate such meal-induced lowering in acetylcarnitine metabolism cannot be deducted from this study. Interestingly, a recently published study in obese mice showed an effect of combined supplementation of NR with L-carnitine and reported a reduction in fat mass percentage and hepatic steatosis (54), which matches with the positive outcome parameters of our and other studies $(9,21,48)$. The exact link between NR metabolism and acetylcarnitine metabolism is however still unknown. Furthermore, the large gap between the clear metabolic improvements upon NR in mice and the lack of effects in humans might derive from the fact that mice studies applied a longer supplementation duration (8 to 15 weeks) $(9,10,12,48)$ compared to short-term supplementation in human trials (3 to 12 weeks) $(21,24,25,55)$.

In conclusion, we here show that NR supplementation of $1000 \mathrm{mg} /$ day for six weeks in healthy overweight and obese men and women increased the NAD+ metabolites NAAD and MeNAM in human skeletal muscle and increased skeletal muscle acetylcarnitine metabolism. In addition, NR induced improvements in body composition and increased sleeping metabolic rate. However, no other metabolic health effects were observed. We conclude that NR, at this dose and short duration, may not be beneficial in improving metabolic health in healthy overweight and obese men and women. However, further research is warranted into the effects of long-term NR supplementation on acetylcarnitine concentrations, sex specific improvements in body composition and metabolic health effects in humans. 


\section{ACKNOWLEDGEMENTS}

The authors' contributions were as follows: CR BH JA JH VSH EP PS designed the study. CR KR MM NC VdW conducted the experiments. $\mathrm{BH}$ provided medical responsibility. SA BS HE RZP performed analysis in plasma samples and muscle biopsies. CR JM VdW TvdW SA RZP VSH analyzed data. CR SA EL RZP RH JH LL VSH EP PS interpreted the data. CR EP PS wrote paper and all authors reviewed and accepted the last version of the manuscript. PS had primary responsibility for final content. The authors would like to thank the participants for their commitment to this study and ChromaDex Inc. for providing NIAGEN and placebo capsules for the study. PS is the guarantor of this work and, as such, had full access to all the data in the study and takes responsibility for the integrity of the data and the accuracy of the data analysis. The authors declare no conflict of interest relevant to this article. 


\section{Chapter 2}

\section{SUPPLEMENTARY MATERIAL FOR CHAPTER 2}

Supplementary Table 1. Internal standard mixture for $\mathrm{NAD}^{+}$targeted metabolomics in skeletal muscle biopsies

\begin{tabular}{|l|l|}
\hline Internal standard & Concentration $(\mu \mathrm{M})$ \\
\hline Adenosine- ${ }^{15} \mathrm{~N}_{5}$-monophosphate & 100 \\
\hline Adenosine-- ${ }^{15} \mathrm{~N}_{5}$-triphosphate & 1000 \\
\hline $\mathrm{D}_{4}$-alanine & 100 \\
\hline $\mathrm{D}_{7}$-arginine & 100 \\
\hline $\mathrm{D}_{3}$-aspartic acid & 100 \\
\hline $\mathrm{D}_{4}$-citric acid & 100 \\
\hline${ }^{13} \mathrm{C}_{1}$-Citrulline & 100 \\
\hline${ }^{13} \mathrm{C}_{6}$-fructose-1,6-diphosphate & 100 \\
\hline Guanosine-15 $\mathrm{N}_{5}$-monophosphate & 100 \\
\hline Guanosine-- $\mathrm{N}_{5}$-triphosphate & 1000 \\
\hline${ }^{13} \mathrm{C}_{6}$-glucose & 1000 \\
\hline${ }^{13} \mathrm{C}_{6}$-glucose-6-phosphate & 100 \\
\hline $\mathrm{D}_{3}$-glutamic acid & 100 \\
\hline $\mathrm{D}_{5}$-glutamine & 100 \\
\hline${ }^{13} \mathrm{C}_{6}$-isoleucine & 100 \\
\hline $\mathrm{D}_{3}$-leucine & 100 \\
\hline $\mathrm{D}_{4}$-lysine & 100 \\
\hline $\mathrm{D}_{3}$-methionine & 100 \\
\hline $\mathrm{D}_{6}$-Ornithine & 100 \\
\hline $\mathrm{D}_{5}$-phenylalanine & 100 \\
\hline $\mathrm{D}_{7}$-proline & 100 \\
\hline${ }^{13} \mathrm{C}_{3}$-pyruvate & 100 \\
\hline $\mathrm{D}_{3}$-Serine & 100 \\
\hline $\mathrm{D}_{5}$-tryptophan & 100 \\
\hline $\mathrm{D}_{4}$-tyrosine & 100 \\
\hline $\mathrm{D}_{8}$-valine & 100 \\
\hline${ }^{13} \mathrm{C}_{5}$-nicotinamide adenine dinucleotide & \\
\hline
\end{tabular}


Supplementary Table 2. Participant characteristics

\begin{tabular}{ll}
\hline Parameter & Mean \pm SD \\
\hline Gender F/M & $7 / 6$ \\
Age (years) & $59 \pm 5$ \\
Body weight (kg) & $87.2 \pm 13.6$ \\
Height $(\mathrm{m})$ & $1.70 \pm 0.11$ \\
BMl $\left(\mathrm{kg} / \mathrm{m}^{2}\right)$ & $30.2 \pm 2.6$ \\
VO ${ }_{2}$ peak $(\mathrm{ml} / \mathrm{min} / \mathrm{kg})$ & $27.0 \pm 5.7$ \\
Physical activity level (Baecke score) & $7.51 \pm 1.16$
\end{tabular}

BMI, body mass index; $\mathrm{VO}_{2}$ peak, peak oxygen consumption. 
Supplementary Figure 1. Flowchart participant inclusion

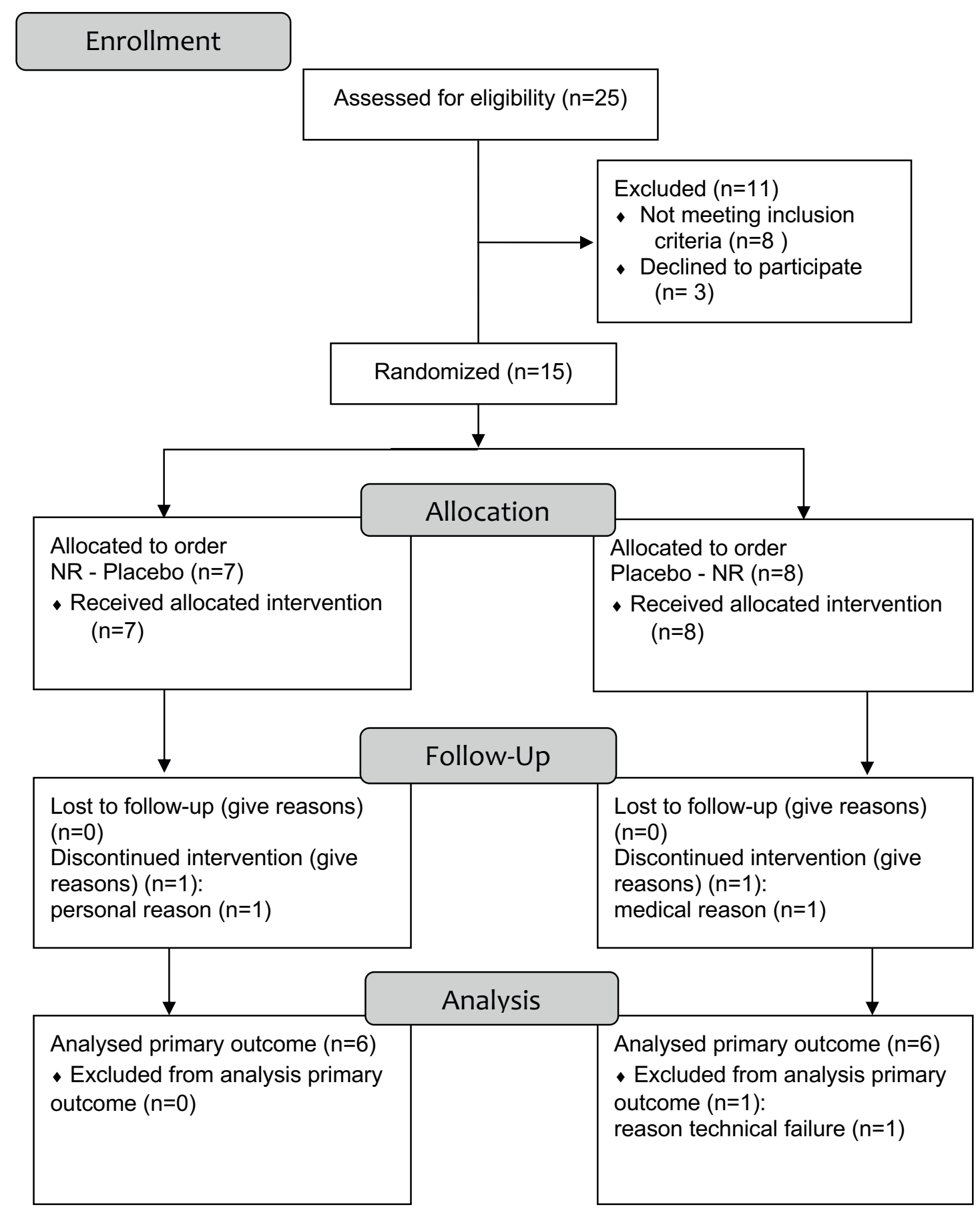




\section{REFERENCES}

1. Bieganowski P, Brenner C. Discoveries of nicotinamide riboside as a nutrient and conserved NRK genes establish a Preiss-Handler independent route to NAD+ in fungi and humans. Cell. 2004;117(4):495-502.

2. Connell NJ, Houtkooper RH, Schrauwen P. NAD(+) metabolism as a target for metabolic health: have we found the silver bullet? Diabetologia. 2019;62(6):888-99.

3. Houtkooper $\mathrm{RH}$, Auwerx J. Exploring the therapeutic space around NAD+. J Cell Biol. 2012;199(2):205-9.

4. Imai S, Guarente L. NAD+ and sirtuins in aging and disease. Trends in cell biology. 2014;24(8):464-71.

5. Bonkowski MS, Sinclair DA. Slowing ageing by design: the rise of $N A D(+)$ and sirtuin-activating compounds. Nat Rev Mol Cell Biol. 2016;17(11):679-90.

6. Canto C, Auwerx J. Targeting sirtuin 1 to improve metabolism: all you need is NAD(+)? Pharmacological reviews. 2012;64(1):166-87.

7. Connell $\mathrm{NJ}$, Houtkooper $\mathrm{RH}$, Schrauwen P. NAD $(+)$ metabolism as a target for metabolic health: have we found the silver bullet? Diabetologia. 2019.

8. Fletcher RS, Ratajczak J, Doig CL, Oakey LA, Callingham R, Da Silva Xavier G, Garten A, Elhassan YS, Redpath P, Migaud ME, et al. Nicotinamide riboside kinases display redundancy in mediating nicotinamide mononucleotide and nicotinamide riboside metabolism in skeletal muscle cells. Mol Metab. 2017;6(8):819-32.

9. Canto C, Houtkooper RH, Pirinen E, Youn DY, Oosterveer MH, Cen Y, Fernandez-Marcos PJ, Yamamoto H, Andreux PA, Cettour-Rose P, et al. The NAD(+) precursor nicotinamide riboside enhances oxidative metabolism and protects against high-fat diet-induced obesity. Cell metabolism. 2012;15(6):838-47.

10. Khan NA, Auranen M, Paetau I, Pirinen E, Euro L, Forsstrom S, Pasila L, Velagapudi V, Carroll CJ, Auwerx J, et al. Effective treatment of mitochondrial myopathy by nicotinamide riboside, a vitamin B3. EMBO molecular medicine. 2014;6(6):721-31.

11. Trammell SAJ, Weidemann BJ, Chadda A, Yorek MS, Holmes A, Coppey LJ, Obrosov A, Kardon RH, Yorek MA, Brenner C. Nicotinamide Riboside Opposes Type 2 Diabetes and Neuropathy in Mice.

12. Shi W, Hegeman MA, van Dartel DAM, Tang J, Suarez M, Swarts H, van der Hee B, Arola L, Keijer J. Effects of a wide range of dietary nicotinamide riboside (NR) concentrations on metabolic flexibility and white adipose tissue (WAT) of mice fed a mildly obesogenic diet. Mol Nutr Food Res. 2017;61(8).

13. Frederick DW, Loro E, Liu L, Davila A, Jr., Chellappa K, Silverman IM, Quinn WJ, 3rd, Gosai SJ, Tichy ED, Davis JG, et al. Loss of NAD Homeostasis Leads to Progressive and Reversible Degeneration of Skeletal Muscle. Cell metabolism. 2016;24(2):269-82.

14. Trammell SA, Schmidt MS, Weidemann BJ, Redpath P, Jaksch F, Dellinger RW, Li Z, Abel ED, Migaud ME, Brenner C. Nicotinamide riboside is uniquely and orally bioavailable in mice and humans. Nature communications. 2016;7:12948.

15. Yoshino J, Mills KF, Yoon MJ, Imai S. Nicotinamide mononucleotide, a key NAD(+) intermediate, treats the pathophysiology of diet- and age-induced diabetes in mice. Cell metabolism. 2011;14(4):528-36.

16. Massudi H, Grant R, Braidy N, Guest J, Farnsworth B, Guillemin GJ. Age-associated changes in oxidative stress and NAD+ metabolism in human tissue. PloS one. 2012;7(7):e42357.

17. de Guia RM, Agerholm M, Nielsen TS, Consitt LA, Sogaard D, Helge JW, Larsen S, Brandauer J, Houmard JA, Treebak JT. Aerobic and resistance exercise training reverses age-dependent decline in $\mathrm{NAD}(+)$ salvage capacity in human skeletal muscle. Physiol Rep. 2019;7(12):e14139.

18. Dellinger RW, Santos SR, Morris M, Evans M, Alminana D, Guarente L, Marcotulli E. Erratum: Author Correction: Repeat dose NRPT (nicotinamide riboside and pterostilbene) increases $\mathrm{NAD}(+)$ levels in humans safely and sustainably: a randomized, double-blind, placebocontrolled study. NPJ Aging Mech Dis. 2017;4:8.

19. Airhart SE, Shireman LM, Risler LJ, Anderson GD, Nagana Gowda GA, Raftery D, Tian R, Shen DD, O'Brien KD. An open-label, non-randomized study of the pharmacokinetics of the nutritional supplement nicotinamide riboside (NR) and its effects on blood NAD+ levels in healthy volunteers. PloS one. 2017;12(12):e0186459. 
20. Martens CR, Denman BA, Mazzo MR, Armstrong ML, Reisdorph N, McQueen MB, Chonchol $\mathrm{M}$, Seals DR. Chronic nicotinamide riboside supplementation is well-tolerated and elevates $\mathrm{NAD}+$ in healthy middle-aged and older adults. Nature communications.9(1286).

21. Dollerup OL, Christensen B, Svart M, Schmidt MS, Sulek K, Ringgaard S, Stodkilde-Jorgensen $\mathrm{H}$, Moller N, Brenner C, Treebak JT, et al. A randomized placebo-controlled clinical trial of nicotinamide riboside in obese men: safety, insulin-sensitivity, and lipid-mobilizing effects. The American journal of clinical nutrition. 2018;108(2):343-53.

22. Conze D, Brenner C, Kruger CL. Safety and Metabolism of Long-term Administration of NIAGEN (Nicotinamide Riboside Chloride) in a Randomized, Double-Blind, Placebo-controlled Clinical Trial of Healthy Overweight Adults. Sci Rep. 2019;9(1):9772.

23. Conze DB, Crespo-Barreto J, Kruger CL. Safety assessment of nicotinamide riboside, a form of vitamin B3. Human \& experimental toxicology. 2016.

24. Dollerup OL, Chubanava S, Agerholm M, Søndergard SD, Altintas A, Møller AB, Høyer KF, Ringgaard S, Stødkilde-Jørgensen H, Lavery GG, et al. Nicotinamide riboside does not alter mitochondrial respiration, content or morphology in skeletal muscle from obese and insulin resistant men. The Journal of physiology. 2019.

25. Elhassan YS, Kluckova K, Fletcher RS, Schmidt MS, Garten A, Doig CL, Cartwright DM, Oakey L, Burley CV, Jenkinson N, et al. Nicotinamide Riboside Augments the Aged Human Skeletal Muscle NAD(+) Metabolome and Induces Transcriptomic and Anti-inflammatory Signatures. Cell reports. 2019;28(7):1717-28 e6.

26. Baecke JA, Burema J, Frijters JE. A short questionnaire for the measurement of habitual physical activity in epidemiological studies. The American journal of clinical nutrition. 1982;36(5):936-42.

27. DeFronzo RA, Tobin JD, Andres R. Glucose clamp technique: a method for quantifying insulin secretion and resistance. Am J Physiol. 1979;237(3):E214-23.

28. Bergstrom J, Hermansen L, Hultman E, Saltin B. Diet, muscle glycogen and physical performance. Acta physiologica Scandinavica. 1967;71(2):140-50.

29. Kato T, Berger SJ, Carter JA, Lowry OH. An enzymatic cycling method for nicotinamideadenine dinucleotide with malic and alcohol dehydrogenases. Analytical biochemistry. 1973;53(1):86-97.

30. van de Weijer T, Phielix E, Bilet L, Williams EG, Ropelle ER, Bierwagen A, Livingstone R, Nowotny P, Sparks LM, Paglialunga S, et al. Evidence for a direct effect of the NAD+ precursor acipimox on muscle mitochondrial function in humans. Diabetes. 2015;64(4):1193-201.

31. Hoeks J, van Herpen NA, Mensink M, Moonen-Kornips E, van Beurden D, Hesselink MK, Schrauwen P. Prolonged fasting identifies skeletal muscle mitochondrial dysfunction as consequence rather than cause of human insulin resistance. Diabetes. 2010;59(9):2117-25.

32. Wefers J, van Moorsel D, Hansen J, Connell NJ, Havekes B, Hoeks J, van Marken Lichtenbelt WD, Duez H, Phielix E, Kalsbeek A, et al. Circadian misalignment induces fatty acid metabolism gene profiles and compromises insulin sensitivity in human skeletal muscle. Proceedings of the National Academy of Sciences of the United States of America. 2018;115(30):7789-94.

33. Kuipers H, Verstappen FT, Keizer HA, Geurten P, van Kranenburg G. Variability of aerobic performance in the laboratory and its physiologic correlates. Int J Sports Med. 1985;6(4):197201.

34. Lindeboom L, Nabuurs Cl, Hesselink MK, Wildberger JE, Schrauwen P, Schrauwen-Hinderling VB. Proton magnetic resonance spectroscopy reveals increased hepatic lipid content after a single high-fat meal with no additional modulation by added protein. The American journal of clinical nutrition. 2015;101(1):65-71.

35. Lindeboom L, Bruls YM, van Ewijk PA, Hesselink MK, Wildberger JE, Schrauwen P, SchrauwenHinderling VB. Longitudinal relaxation time editing for acetylcarnitine detection with (1) H-MRS. Magn Reson Med. 2017;77(2):505-10.

36. Lindeboom L, Nabuurs Cl, Hoeks J, Brouwers B, Phielix E, Kooi ME, Hesselink MK, Wildberger JE, Stevens RD, Koves T, et al. Long-echo time MR spectroscopy for skeletal muscle acetylcarnitine detection. The Journal of clinical investigation. 2014;124(11):4915-25.

37. van de Weijer T, van Ewijk PA, Zandbergen HR, Slenter JM, Kessels AG, Wildberger JE, Hesselink MK, Schrauwen P, Schrauwen-Hinderling VB, Kooi ME. Geometrical models for cardiac MRI in rodents: comparison of quantification of left ventricular volumes and function by various geometrical models with a full-volume MRI data set in rodents. Am J Physiol Heart Circ Physiol. 2012;302(3):H709-15.

38. Joris PJ, Plat J, Bakker SJ, Mensink RP. Long-term magnesium supplementation improves arterial stiffness in overweight and obese adults: results of a randomized, double-blind, 
placebo-controlled intervention trial. The American journal of clinical nutrition. 2016;103(5):1260-6.

39. Dempster P, Aitkens S. A new air displacement method for the determination of human body composition. Med Sci Sports Exerc. 1995;27(12):1692-7.

40. Plasqui G, Soenen S, Westerterp-Plantenga MS, Westerterp KR. Measurement of Iongitudinal changes in body composition during weight loss and maintenance in overweight and obese subjects using air-displacement plethysmography in comparison with the deuterium dilution technique. Int J Obes (Lond). 2011;35(8):1124-30.

41. Friedewald WT, Levy RI, Fredrickson DS. Estimation of the concentration of low-density lipoprotein cholesterol in plasma, without use of the preparative ultracentrifuge. Clin Chem. 1972;18(6):499-502.

42. Peronnet F, Massicotte D. Table of nonprotein respiratory quotient: an update. Can J Sport Sci. 1991;16(1):23-9.

43. Weir JB. New methods for calculating metabolic rate with special reference to protein metabolism. The Journal of physiology. 1949;109(1-2):1-9.

44. Steele R. Influences of glucose loading and of injected insulin on hepatic glucose output. Annals of the New York Academy of Sciences. 1959;82:420-30.

45. de Ligt M, Bruls YMH, Hansen J, Habets MF, Havekes B, Nascimento EBM, Moonen-Kornips E, Schaart G, Schrauwen-Hinderling VB, van Marken Lichtenbelt W, et al. Resveratrol improves ex vivo mitochondrial function but does not affect insulin sensitivity or brown adipose tissue in first degree relatives of patients with type 2 diabetes. Mol Metab. 2018;12:39-47.

46. Timmers S, Konings E, Bilet L, Houtkooper RH, van de Weijer T, Goossens GH, Hoeks J, van der Krieken S, Ryu D, Kersten S, et al. Calorie restriction-like effects of 30 days of resveratrol (resVida) supplementation on energy metabolism and metabolic profile in obese humans. Cell metabolism. 2011;14(5).

47. Timmers S, de Ligt M, Phielix E, van de Weijer T, Hansen J, Moonen-Kornips E, Schaart G, Kunz I, Hesselink MK, Schrauwen-Hinderling VB, et al. Resveratrol as Add-on Therapy in Subjects With Well-Controlled Type 2 Diabetes: A Randomized Controlled Trial. Diabetes care. 2016;39:2211-7.

48. Trammell SA, Weidemann BJ, Chadda A, Yorek MS, Holmes A, Coppey LJ, Obrosov A, Kardon $\mathrm{RH}$, Yorek MA, Brenner C. Nicotinamide Riboside Opposes Type 2 Diabetes and Neuropathy in Mice. Sci Rep. 2016;6:26933.

49. Diguet N, Trammell SAJ, Tannous C, Deloux R, Piquereau J, Mougenot N, Gouge A, Gressette M, Manoury B, Blanc J, et al. Nicotinamide Riboside Preserves Cardiac Function in a Mouse Model of Dilated Cardiomyopathy. Circulation. 2018;137(21):2256-73.

50. Verdecchia P, Angeli F, Cavallini C. Ambulatory blood pressure for cardiovascular risk stratification. Circulation. 2007;115(16):2091-3.

51. Hansen TW, Jeppesen J, Rasmussen S, Ibsen H, Torp-Pedersen C. Ambulatory blood pressure monitoring and risk of cardiovascular disease: a population based study. Am J Hypertens. 2006; 19(3):243-50.

52. Klepochova R, Valkovic L, Gajdosik M, Hochwartner T, Tschan H, Krebs M, Trattnig S, Krssak M. Detection and Alterations of Acetylcarnitine in Human Skeletal Muscles by $1 \mathrm{H} \mathrm{MRS} \mathrm{at} 7$ T. Invest Radiol. 2017;52(7):412-8.

53. Watt MJ, Heigenhauser GJ, Stellingwerff T, Hargreaves M, Spriet LL. Carbohydrate ingestion reduces skeletal muscle acetylcarnitine availability but has no effect on substrate phosphorylation at the onset of exercise in man. The Journal of physiology. 2002;544(3):94956.

54. Salic K, Gart E, Seidel F, Verschuren L, Caspers M, van Duyvenvoorde W, Wong KE, Keijer J, Bobeldijk-Pastorova I, Wielinga PY, et al. Combined treatment with I-carnitine and nicotinamide riboside improves hepatic metabolism and attenuates obesity and liver steatosis. International journal of molecular sciences. 2019;20:4359.

55. Martens CR, Denman BA, Mazzo MR, Armstrong ML, Reisdorph N, McQueen MB, Chonchol $\mathrm{M}$, Seals DR. Chronic nicotinamide riboside supplementation is well-tolerated and elevates $\mathrm{NAD}(+)$ in healthy middle-aged and older adults. Nature communications. 2018;9(1):1286. 



\section{CHAPTER 3}

Increasing $\mathrm{NAD}^{+}$levels through nicotinamide riboside activates in vivo brown adipose tissue in mice but not in humans

Emmani B.M. Nascimento*, Michiel P.B. Moonen*, Carlijn M.E. Remie, Karim Gariani, Johanna A. Jörgensen, Gert Schaart, Johan Auwerx, Wouter D. van Marken Lichtenbelt, Patrick Schrauwen

* These authors contributed equally

Submitted

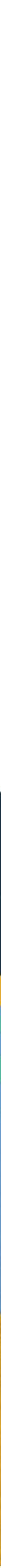




\title{
Chapter 3
}

\begin{abstract}
Elevating $\mathrm{NAD}^{+}$levels systemically improves metabolic health, which can be accomplished via nicotinamide riboside (NR). Previously, it was demonstrated that NR supplementation in high fat diet (HFD)-fed mice decreased weight gain, normalized glucose metabolism and enhanced cold tolerance. As brown adipose tissue (BAT) is a major source of thermogenesis, we hypothesize that NR stimulates BAT in mice and humans. HFD-fed mice were supplemented with $400 \mathrm{mg} / \mathrm{kg} /$ day NR and subsequently exposed to cold. Next, in vitro primary adipocytes derived from human BAT biopsies were pretreated with $50 \mu \mathrm{M}$ or $500 \mu \mathrm{M}$ NR prior to measuring mitochondrial uncoupling. Finally, human volunteers were supplemented with 1000 $\mathrm{mg} /$ day NR for six weeks to determine whether BAT activity increased, as measured by [18F]-FDG uptake via PET-CT. NR supplementation in HFD-fed mice decreased BAT mass and adipocyte cell size. Cold exposure further decreased adipocyte cell size on top of that achieved by NR alone. In adipocytes derived from human BAT, NR enhanced in vitro norepinephrine (NE)-stimulated mitochondrial uncoupling. However, NR supplementation in human volunteers did not alter BAT activity or cold induced thermogenesis. Our research demonstrates the need for translational research in order to better understand the differences in $\mathrm{NAD}^{+}$metabolism between mouse and human.
\end{abstract}




\section{INTRODUCTION}

Brown adipose tissue (BAT) has been proposed as a promising target to stimulate energy expenditure in humans with obesity or type 2 diabetes. The presence of uncoupling protein 1 (UCP1) in mitochondria of BAT enables heat production instead of ATP synthesis while using glucose and fatty acids as fuel (1). The interest in human BAT was sparked by the discovery of active BAT in adult humans (2-5) and high BAT activity is associated with healthy whole-body metabolism. Cold exposure is the most effective way to stimulate BAT activity in humans (6). Prolonged cold exposure may, however, not be an attractive treatment option for many people and thus pharmacological alternatives are actively explored. In this context, we and others have shown that bile acids stimulate BAT activity in mice (7) and healthy humans (8), however, it is not known whether this approach is effective in human metabolic disease. In addition of its direct effect of BAT, bile acids have recently been showed to induce beiging of the subcutaneous white adipose tissue (3) through the bile acidresponsive membrane receptor TGR5 (9). This would enhance the thermogenic activity of WAT and promote the use of glucose and fatty acids for mitochondrial uncoupling, which is relevant in humans with disturbed glucose and/or fatty acid metabolism (e.g. obesity, type 2 diabetes).

Another approach to activate BAT involves activation of the beta-adrenergic receptor via administration of agonists, like mirabegron. However, beta-adrenergic stimulation at doses high enough to activate BAT also affect cardiac function by increasing heart rate and blood pressure, due to beta-adrenergic receptors in the heart (10). Therefore, other more specific and safer pharmacological strategies are warranted that stimulate BAT activity in humans.

The vitamin $\mathrm{B} 3$ analogue nicotinamide ribose $(N R)$ is a $N A D^{+}$precursor that has been shown to increase the activity of sirtuin (SIRT) 1 and 3 (11). In vitro, NR boosts NAD ${ }^{+}$ levels (12) also resulting in elevated SIRT1/3 activity in vivo (11). In obesity models, NR protected high fat diet (HFD)-fed mice from weight gain and glucose intolerance $(11,13)$. Furthermore, NR supplementation in various rodent models was able to counteract harmful effects of brain damage (14), Alzheimer's disease (15), senescence (16) and muscular dystrophy through increased mitochondrial function (17). NR supplementation also stimulated mitochondrial biogenesis in muscle and BAT in a mouse model of mitochondrial myopathy (18). Interestingly, in mice NR safeguards body temperature following a cold challenge (11) thus implicating the involvement of BAT. These findings give rise to two additional questions: (1.) Is betaadrenergic stimulated BAT activity altered in mice in vivo and in cultured adipocytes 
derived from human BAT following NR?; and (2.) Does NR enhance BAT activity in humans?

Therefore, we here assessed how NR affects BAT in a in vivo HFD-fed mouse model. Since murine BAT is different from human BAT and the effect of NAD+ boosting on human BAT remain unclear, we also investigated the effects of NR on human BAT by using in vitro primary cultured adipocytes derived from human BAT biopsies. Finally, we performed a first human clinical trial in which we supplemented human volunteers with $1000 \mathrm{mg} /$ day NR in order to examine the effects on in vivo BAT activity in humans.

\section{METHODS}

\section{Animal experiments}

Male C57BL/6J mice were purchased from Charles River Laboratories (Wilmington, MA) and were housed under a 14-hour light, 10-hour dark cycle at $21^{\circ} \mathrm{C}$ and had ad libitum access to water and food throughout the experiment. From the age of eight weeks, mice were split into four groups of ten animals. All animals received a high fat diet (D12492) for four weeks from Research Diets Inc. (New Brunswick, NJ). Half of animals were fed with pellets containing vehicle (double-distilled water; $\mathrm{dd}_{2} \mathrm{O}$ ) or NRsupplemented by providing NR (400 mg/kg/day) for four weeks as previously described (19). Mouse body weight was assessed weekly. Cold test was performed as described (20) in seven animals on HF diet alone and in seven animals receiving HF and NR. The rest of the animals was maintained at room temperature. In cold-test groups, mice were anesthetized with sodium pentobarbital after six hours of cold exposure (i.p. injection, $50 \mathrm{mg} / \mathrm{kg}$ body weight) and euthanized by blood sampling via cardiac puncture). In the RT group, mice were anesthetized at the same time with sodium pentobarbital (i.p. injection, $50 \mathrm{mg} / \mathrm{kg}$ body weight) and euthanized by blood sampling via cardiac puncture). BAT was snap frozen in liquid nitrogen stored at -80 ${ }^{\circ} \mathrm{C}$. For imaging purposes, BAT was frozen in melting isopentane or stored in $4 \%$ formaldehyde. All animal experiments were carried according to national Swiss and European Union ethical guidelines and approved by the local animal experimentation committee of the Canton de Vaud under license \#2868.

\section{Lipolysis assay}

Murine BAT was isolated and tissue explants were minced and incubated with lipolysis medium at $37{ }^{\circ} \mathrm{C}$ and $5 \% \quad \mathrm{CO}_{2}$. After 2 hours, media was collected and incubated for of five minutes at $37^{\circ} \mathrm{C}$ with free glycerol reagent from Sigma-Aldrich (St. Louis, MO). Following absorption was measured at $540 \mathrm{~nm}$ as previously 
described (21). In cultured adipocytes derived from human BAT and WAT, glycerol release was determined using the enzyChrom ${ }^{\mathrm{TM}}$ adipolysis assay kit from BioAssay systems (Hayward, CA) according to the manufacturer's instructions.

\section{Histology}

BAT samples were fixed in buffered $4 \%$ formaldehyde overnight and embedded in paraffin. $5 \mu \mathrm{m}$ thick serial sections were made from paraffin embedded tissue then stained with hematoxylin and eosin (HE). Another set of BAT samples were embedded in Thermo Scientific ${ }^{\mathrm{TM}}$ Shandon ${ }^{\mathrm{TM}}$ Cryomatrix ${ }^{\mathrm{TM}}$ embedding resin and snap-frozen, for two minutes in isopentane cooled in liquid nitrogen, before being placed on dry ice. $10 \mu \mathrm{m}$ cryosection were cut from biopsies frozen in isopentane. Sections were blocked prior to permeabilization with $0.5 \%$ triton $X-100$. Following sections were incubated with antibodies against UCP1 or TOMM20 from Abcam (Cambridge, United Kingdom). After incubation with the appropriate secondary antibody, sections were mounted using Mowiol prior to imaging.

\section{Culture of human primary adipocytes}

The collection of BAT and WAT biopsies in patients was reviewed and approved by the ethics committee of Maastricht University Medical Center (METC 10-3-012, NL31367.068.10, NCT03111719). Isolation of the stromal vascular fraction and differentiation of cultured adipocytes derived from human BAT and WAT have been described previously (8). In short, collected cells from the stromal vascular fraction were grown to confluence. Differentiation was initiated by a cocktail containing biotin (33 $\mu \mathrm{M})$, pantothenate $(17 \mu \mathrm{M})$, insulin (100 nM), dexamethasone (100 nM), IBMX (250 $\mu \mathrm{M})$, rosiglitazone $(5 \mu \mathrm{M})$, T3 $(2 \mathrm{nM})$ and transferrin $(10 \mu \mathrm{g} / \mathrm{ml})$. Cells were transferred to maintenance medium consisting of biotin $(33 \mu \mathrm{M})$, pantothenate $(17 \mu \mathrm{M})$, insulin (100 nM), dexamethasone (10 nM), T3 (2 nM) and transferrin $(10 \mu \mathrm{g} / \mathrm{ml})$ until lipidaccumulating adipocytes had formed.

\section{Mitochondrial respiration}

Mitochondrial respiration in cultured adipocytes derived from human BAT and WAT has been described before (8). In short, adipocytes derived from human BAT and WAT were differentiated in XF96 well plates. Oxygen consumption rates were measured using the XF96 extracellular flux analyzer from Seahorse Biosciences (North Billerica, MA). Cells were incubated for 1 hour at $37^{\circ} \mathrm{C}$ in unbuffered DMEM (2 $\mathrm{mM}$ GlutaMAX, $1 \mathrm{mM}$ sodium pyruvate and $25 \mathrm{mM}$ glucose). Basal oxygen consumption was measured followed by injection of $2 \mu \mathrm{M}$ oligomycin subsequently followed by injection of the compounds of interest. When indicated cells were 


\section{Chapter 3}

preincubated with NR for 24 hours prior to the start of the experiment. Data is plotted as a percentage compared to uncoupled respiration following oligomycin.

\section{RNA isolation and gene expression analysis}

Total RNA was extracted from cultured adipocytes derived from human BAT and WAT using the miRNEasy kit from Qiagen (Hilden, Germany) according to the manufacturer. cDNA was created using the high capacity RNA-to-cDNA-kit from Applied Biosystems (Foster City, CA). Gene expression data was normalized to TATA box-binding protein (TBP) and further analyzed using the $2^{-\Delta \Delta \mathrm{Ct}}$ method. Primers for SIRT3 (Hs00953477_m1), SOD2 (Hs00167309_m1) and UCP1 (Hs00222453_m1) were from Applied Biosystems (Foster City, CA). SYBR-green qPCR primers for PGC1a: forward primer 5'-TGCTGAAGAGGGAAAGTGAGCGATTAGTTGA-3', reverse primer 5'-AGGTGAAAGTGTAATACTGTTGGTTGA-3'; SIRT1: forward primer 5'- AGAGCCTCACATGCAAGCTCTAG-3', reverse primer 5'GCCAATCATAAGATGTTGCTGAAC-3'; TFAM: forward primer 5'TTCCCAAGACTTCATTTCATTGTC-3', $\quad$ reverse 5rimer GATGATTCGGCTCAGGGAAA-3'. Primers for TBP have been described previously (22).

\section{Western blot analysis}

Protein was extracted using lysis buffer (50 mM Tris, 1 mM EDTA, 1\% NP40, 5 mM nicotinamide, $1 \mathrm{mM}$ sodium butyrate, $150 \mathrm{mM} \mathrm{KCl}$, protease inhibitors $[\mathrm{pH} 7.4]$ ). Proteins were separated by SDS-PAGE and transferred onto nitrocellulose membranes. Blocking and antibody incubations were performed in 5\% BSA. UCP1 antibody was from Abcam (Cambridge, UK). Antibody detection reactions were developed by enhanced chemiluminescence from Advansta (Menlo Park, CA) using imaged using the c300 imaging system from Azure Biosystems (Dublin, CA). Cultured adipocytes derived from human BAT and WAT were harvested in Laemmli buffer. Proteins were separated using SDS-PAGE followed by transfer to nitrocellulose membranes. Proteins were detected and quantified using the Odyssey from LI-COR Biosciences. Antibodies against UCP1 and OXPHOS were purchased from Abcam (Cambridge, United Kingdom). Beta-actin (A5316) was detected using an antibody from Sigma-Aldrich (St. Louis, MO).

\section{$\mathrm{NAD}^{+}$determination}

$\mathrm{NAD}^{+}$concentrations were determined using the enzyChrom ${ }^{\mathrm{TM}} \mathrm{NAD}^{+} / \mathrm{NADH}$ assay kit from BioAssay Systems (Hayward, CA) according to the manufacturer's instructions. 


\section{ATP determination}

ATP concentrations were determined using the CellTiter-Glo® Luminescent cell viability assay from Promega (Madison, WI) according to the manufacturer's instructions.

\section{Clinical trial in humans}

The ethics committee of Maastricht University Medical Center approved the study protocol (METC 16-30-19, NL58119.068.16, NCT02835664) and all volunteers provided written informed consent. Eight healthy overweight and obese men and postmenopausal women were recruited. Inclusion criteria were 45-65 years of age, BMI 27-35 kg/m², sedentary lifestyle ( $<3$ h exercise per week), non-smoking for at least 6 months, no alcohol use of $>2$ servings per day, stable body weight for at least 6 months and no active diseases. A randomized controlled, double blinded, placebo controlled, cross-over study with NR supplementation was performed. Volunteers underwent two times a six-week period with oral supplementation of either NR 1000 mg daily (NIAGEN, Chromadex) or placebo (capsules identical to NR in external appearance and number). Supplements were consumed with breakfast (500 mg) and lunch (500 mg). Measurements were performed on the last day of each supplementation period. Participants were fasted for at least 11 hours when entering the test facilities. For the determination of cold-induced BAT activity, volunteers were wrapped in a water-perfused suit (ThermaWrap Universal 3166; MTRE Advanced Technologies Ltd., Yavne, Israel). First, volunteers remained at thermoneutral conditions (32 ${ }^{\circ} \mathrm{C}$ water) for 30 minutes, during which basal metabolic rate was measured by indirect calorimetry (IDEE, Maastricht Instruments, Maastricht, The Netherlands). Thereafter an individualized cooling procedure was started to determine NST as described before (23). In brief, volunteers were cooled down in order to maximize NST after which energy expenditure was measured for 30 minutes. Following, $75 \mathrm{MBq}$ of [18F]-FDG was injected via the intravenous canula. Cold exposure was continued for 60 minutes, while volunteers were instructed to remain lying still. Next, the volunteers were unwrapped and underwent a static [18F]-FDGPET/CT scan (Gemini TF PET-CT, Philips, The Netherlands). This consisted of a lowdose CT scan (120 kV, 30 mAs) followed by a PET scan. Six to seven bed positions (5 minutes per bed position) were used, to cover te area from the skull to the iliac crest. Tracer uptake was determined with the PET scan while the CT was used for attenuation correction and anatomical localization of the active BAT. The scans were analyzed with PMOD software (version 3.0; PMOD Technologies). The regions of interest were manually outlined, while a threshold of 1.5 SUV (standardized uptake value) and Hounsfield units between -10 en -180 were used, as described previously by our group (23). Additionally fixed volumes (10 $\mathrm{mm}$ by $10 \mathrm{~mm}$ ) were placed in the 


\section{Chapter 3}

cervical adipose tissue behind the clavicula to measure general uptake values as described before (24). BAT activity was expressed in SUV ([18F]-FDG uptake $(\mathrm{kBq} / \mathrm{mL} /$ (injected dose $(\mathrm{kBq} /$ patient weight $(\mathrm{g})$ ). The activity was determined as average SUV (SUVmean) and as total SUV (SUVmean times the volume of interest).

\section{Statistics}

Two means were compared using student's t-test, or with the Wilcoxon-signed rank test in case of non-parametric data. Comparison of multiple means was assessed by ANOVA. $p<0.05$ was considered statistically significant. Analyses were performed using Graph Pad Prism (San Diego, CA).

\section{RESULTS}

Thermogenesis is enhanced in mice fed a HFD supplemented with NR

NR has been shown to have metabolic effects especially in metabolically challenged animal models. Therefore, to examine BAT metabolism following NR supplementation, we utilized an in vivo mouse model of early insulin resistance and obesity induced by HFD feeding (25). Thus, C57BL/6J mice were fed a HFD for 4 weeks supplemented with or without NR at a concentration of $400 \mathrm{mg} / \mathrm{kg} /$ day. NR supplementation decreased BAT mass compared to control (Figure 1A), indicative of increased BAT activity. Next, animals were challenged with cold $\left(4^{\circ} \mathrm{C}\right)$ for $6 \mathrm{hrs}$. Animals on a HFD showed a significant drop in body temperature following acute cold exposure; however, this decrease in body temperature was prevented by supplementation of NR to HFD (Figure 1B).

\section{NR does not affect ex vivo lipolysis in murine BAT}

In order to examine whether NR supplementation affects lipolysis in BAT in mouse, we performed ex vivo lipolysis experiments using mouse BAT harvested at room temperature $\left(21^{\circ} \mathrm{C}\right)$ and after acute cold exposure $\left(4^{\circ} \mathrm{C}\right)$. At room temperature, supplementation of NR to a HFD did not alter lipolysis (Figure 1C). Also, under conditions of acute cold exposure, supplementation of NR did not significantly change lipolysis in BAT (Figure 1C). UCP1 protein abundance remained unaltered following NR supplementation. Also, the addition of acute cold did not change UCP1 protein expression in mouse BAT (Figure 1D). 


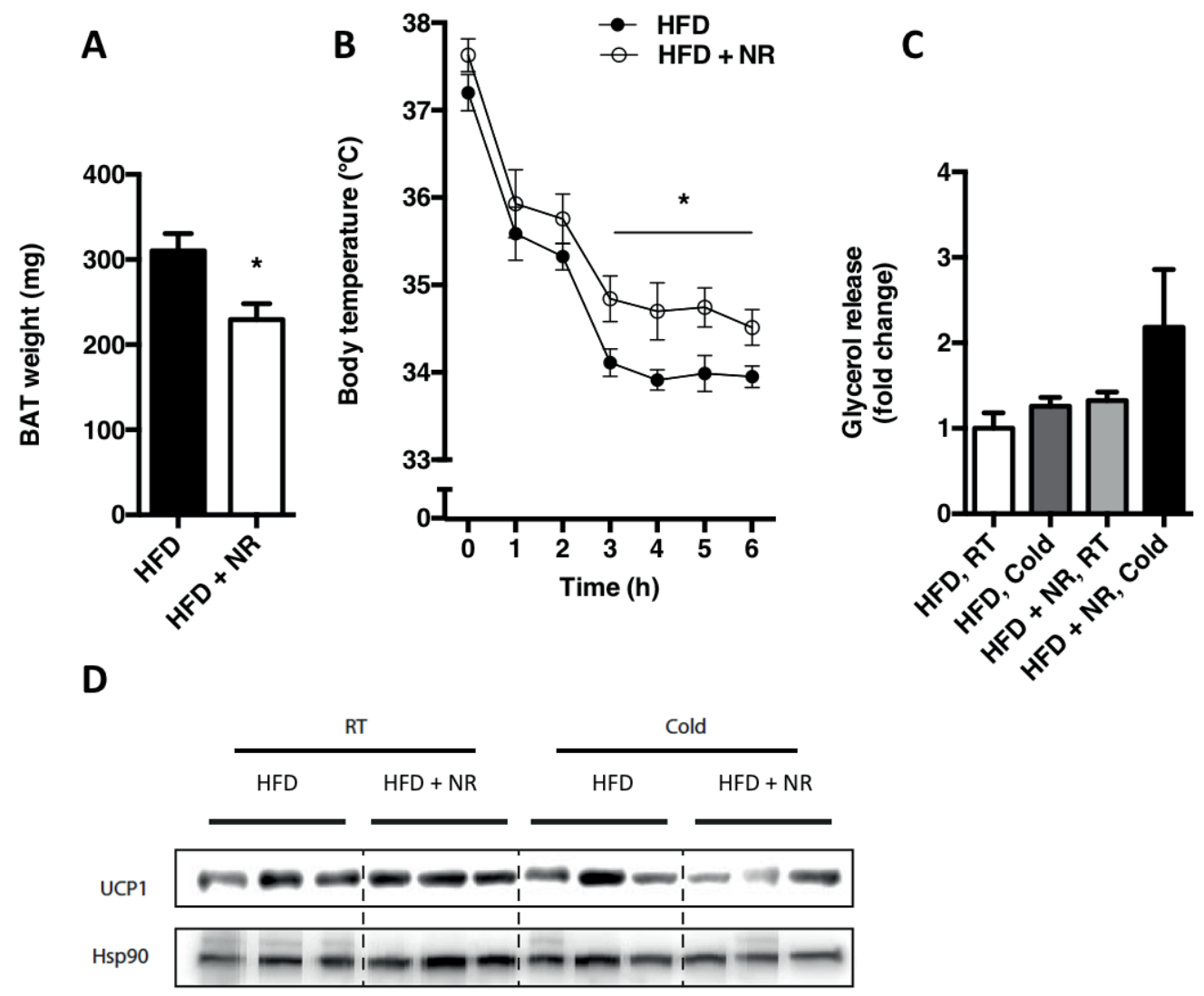

Figure 1. NR stimulates BAT metabolism in mice fed a high fat diet. Mice were fed a high fed diet (HFD) or a high fat diet supplemented with NR (HFD+NR). Mice were kept at room temperature (RT) or acutely cold exposed for six hours (cold). A. BAT mass was weighed at the end of intervention. B. Body temperature was measured for six hours during cold exposure in mice fed a high fat diet (HFD) or mice fed a high fat diet supplemented with NR (HFD+NR). C. Ex vivolipolysis was performed on BAT explants. D. UCP1 protein in mouse BAT. Data is expressed as mean \pm SE. ${ }^{\star} p<0.05(n=5-7)$.

\section{NR combined with cold exposure decreases lipid droplet size in murine BAT}

Based on our finding that NR decreased BAT mass, we next sectioned BAT and stained it with HE in order to get more understanding of BAT morphology and adipocyte size. Brown adipocyte cell size decreased following NR supplementation to HFD (Figure 2A and B). Likewise, acute cold exposure decreased adipocyte cell size. NR supplementation and cold combined resulted in the smallest adipocyte cell size (Figure $2 \mathrm{~A}$ and $\mathrm{B}$ ), indicating active BAT via increase lipolysis. In order to test the involvement of UCP1, we examined UCP1 via immunofluorescence in murine BAT by, which was unaltered following NR supplementation or cold treatment (Figure 2C) matching the data obtained by western blot analysis of protein expression (Figure 1D). Also, mitochondrial density examined by TOMM20 immunofluorescence was unchanged after NR supplementation or cold stimulation (Figure 2D). 


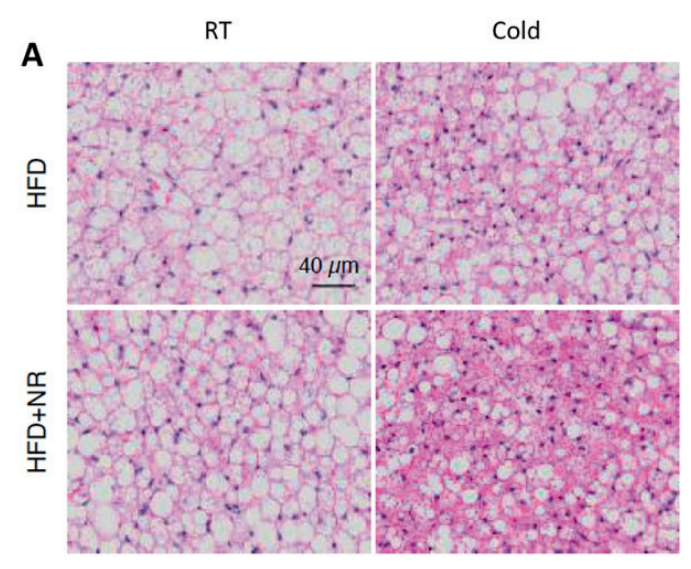

B
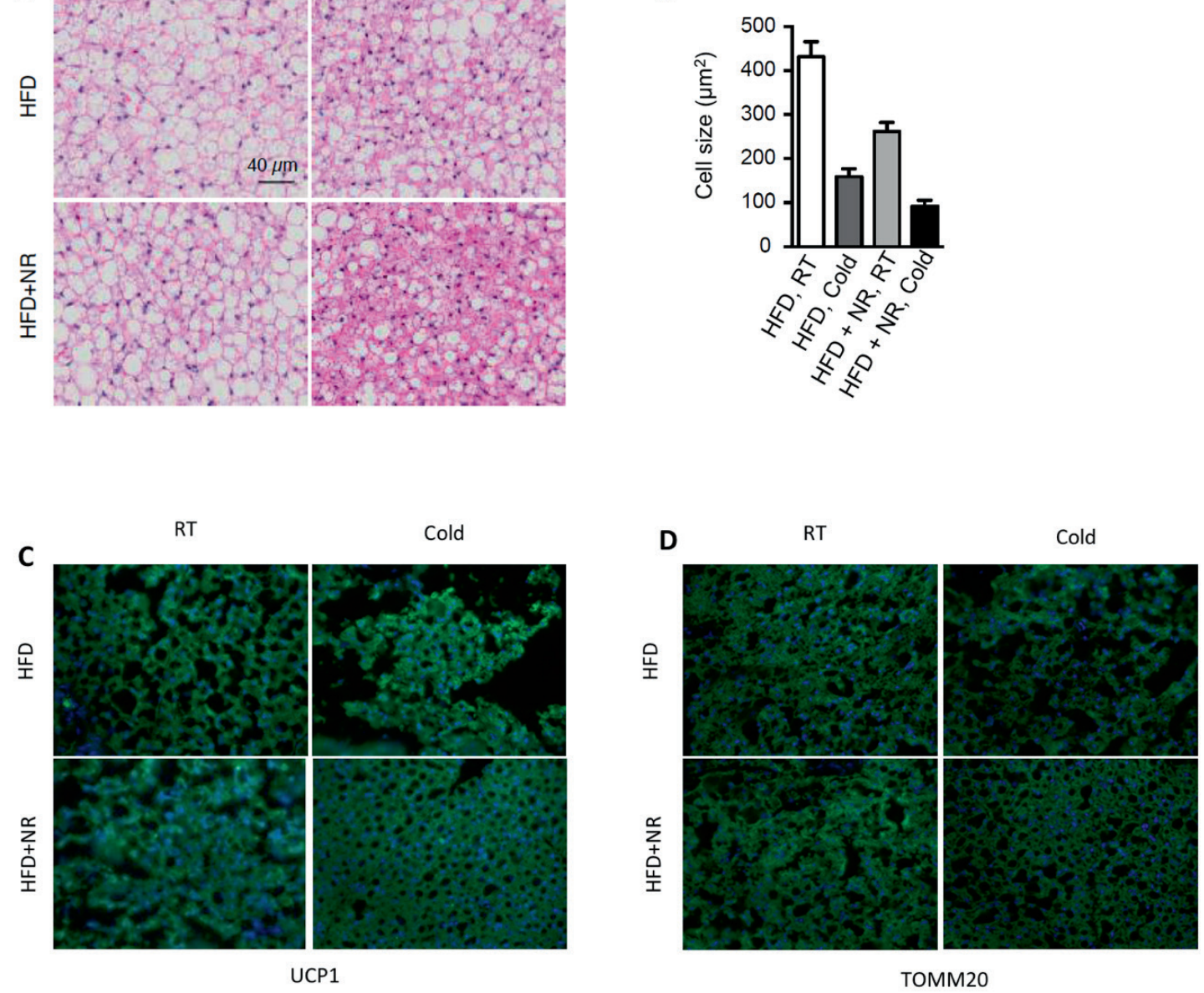

Figure 2. NR supplementation alters adipocyte morphology in mouse BAT following a high fat diet. Mice were fed a high fat diet (HFD) or a high fat diet supplemented with NR (HFD+NR). Following the four-week diet, animals were acutely exposed to cold (cold) or remained at room temperature (RT). A. HE staining of BAT. B. Cell size quantification of results obtained in panel A. C. Immunofluorescent staining of UCP1 in mouse BAT (green). Nuclei were stained with DAPI (blue). D. Immunofluorescent staining of TOMM20 in mouse BAT (green). Nuclei were stained with DAPI (blue). Data is expressed as mean \pm SE $(n=2-3)$.

NR stimulates NE-stimulated mitochondrial uncoupling only in adipocytes derived from human BAT

To investigate if the positive effects of NR on BAT activity in mouse can be translated to humans, we incubated cultured adipocytes derived from human BAT and WAT with 50 or $500 \mu \mathrm{M} N R$ to examine effects on NE-induced mitochondrial uncoupling. Whereas $50 \mu \mathrm{M}$ NR did not increase NAD+ levels, $500 \mu \mathrm{M}$ NR significantly increased $N A D^{+}$levels in adipocytes derived from human BAT and WAT (Figure $3 A$ and $B$ ). Both doses of NR were without effect on ATP concentrations in adipocytes derived from human BAT or WAT (Figure $3 \mathrm{C}$ and $\mathrm{D}$ ). To further investigate the effect on mitochondrial uncoupling in adipocytes derived from BAT and WAT, cultured adipocytes were treated with oligomycin to block ATPase and subsequently 

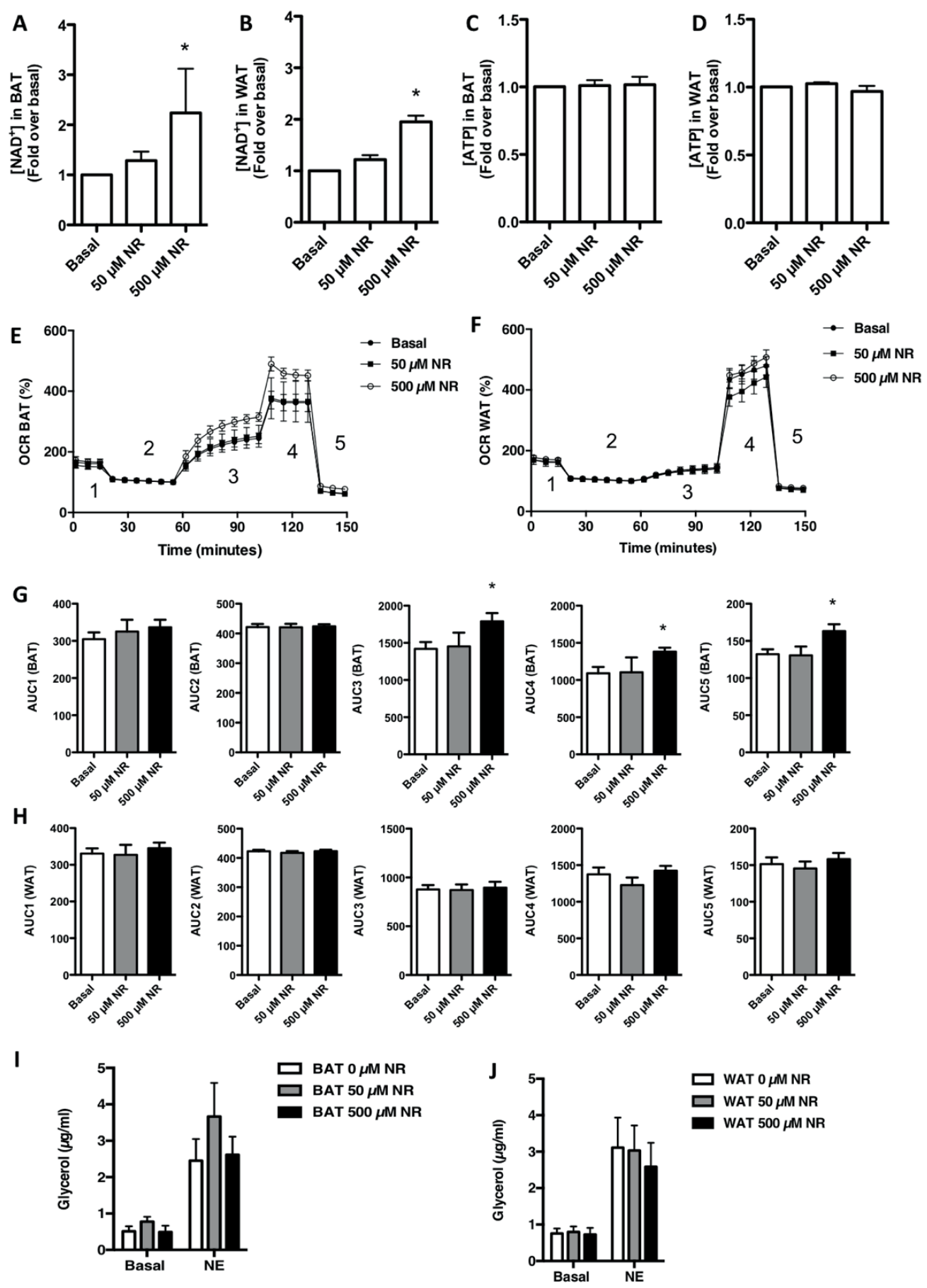

Figure 3. NR stimulates mitochondrial uncoupling in cultured primary adipocytes derived from human BAT. NAD+ (A, B) and ATP (C, D) levels were determined in cultured adipocytes derived from human BAT or WAT after 24 hours with $0 \mu \mathrm{M}, 50 \mu \mathrm{M}$ or $500 \mu \mathrm{M}$ NR. Data is expressed as a fold increase compared to $0 \mu \mathrm{M} N R$. Cellular respiration was measured using the Seahorse bioanalyzer in cultured adipocytes derived from human BAT (E) and WAT (F) following 24 hour incubation with $0 \mu \mathrm{M}, 50 \mu \mathrm{M}$ or $500 \mu \mathrm{M}$ NR. Mitochondrial respiration was measured following injections with oligomycin, NE and FCCP. G. Quantification of AUC from results in panel E. H. Quantification of AUC from results in panel F. NR does not alter lipolysis in cultured adipocytes derived from human BAT. Cultured adipocytes derived from human BAT (I) and WAT (J) were incubated for 24 hours with $0 \mu \mathrm{M}, 50 \mu \mathrm{M}$ or $500 \mu \mathrm{M}$ NR. Following adipocytes were stimulated with $\mathrm{NE}$ in order to measure glycerol release as a marker of lipolysis. Data is expressed as mean $\pm \mathrm{SE}$. ${ }^{*} \mathrm{p}<0.05(\mathrm{n}=4-6)$ 


\section{Chapter 3}

stimulated with NE. NE enhanced mitochondrial uncoupling in adipocytes derived from BAT, while this response was negligible in adipocytes derived from human WAT (Figure 3E and F). $50 \mu \mathrm{M}$ and $500 \mu \mathrm{M}$ NR were unable to potentiate NE-stimulated mitochondrial uncoupling in WAT (Figure 3F and $\mathrm{H}$ ). In human primary brown adipocytes however, 500 MM NR increased NE-stimulated mitochondrial uncoupling (Figure 3E and $\mathrm{G}$ ), as well as maximal FCCP-induced mitochondrial respiration (Figure 3E and $\mathrm{G}$ ). At $500 \mu \mathrm{M} N R$, antimycin $A$ and rotenone resulted in higher OCR compared to $0 \mu \mathrm{M} N R$ and $50 \mu \mathrm{M} N R$.

NR does not affect lipolysis in cultured adipocytes derived from human BAT and WAT To examine if the effect of NR on mitochondrial uncoupling in human BAT cells was mediated via elevated lipolysis, we examined lipolysis following NR treatment in cultured adipocytes derived from human BAT and WAT. Both basal and NEstimulated lipolysis was unchanged following NR treatment in adipocytes derived from human BAT and WAT (Figure $3 \mathrm{I}$ and $\mathrm{J}$ ).

\section{NR does not change gene expression in adipocytes derived from human BAT}

SIRTs are $\mathrm{NAD}^{+}$-dependent deacetylases and NR stimulates SIRT activity by increasing the level of its obligatory co-substrate $\mathrm{NAD}^{+}$(26). Next we assessed whether NR was able to alter gene expression of SIRT-related pathways. Adipocytes derived from human BAT and WAT were incubated with vehicle, $50 \mu \mathrm{M}$ NR or $500 \mu \mathrm{M}$ NR. In cultured adipocytes derived from human WAT, $500 \mu \mathrm{M}$ NR increased transcript levels of Tfam compared to vehicle (Figure 4F). In cultured adipocytes derived from human BAT, NR did not significantly change the transcript levels of any of the genes assessed (UCP1, SIRT3, SOD2, PGC1A or TFAM; Figure 4A-F). We also determined the levels of OXPHOS proteins by western blot examining the individual complexes in adipocytes derived from human BAT and WAT. The highest concentration of $500 \mu \mathrm{M}$ NR decreased the amount of complex I (Figure 4G) in adipocytes derived from human BAT, while $50 \mu \mathrm{M}$ NR increased protein abundance of complex I in adipocytes derived from human WAT (Figure $4 \mathrm{H}$ ). 

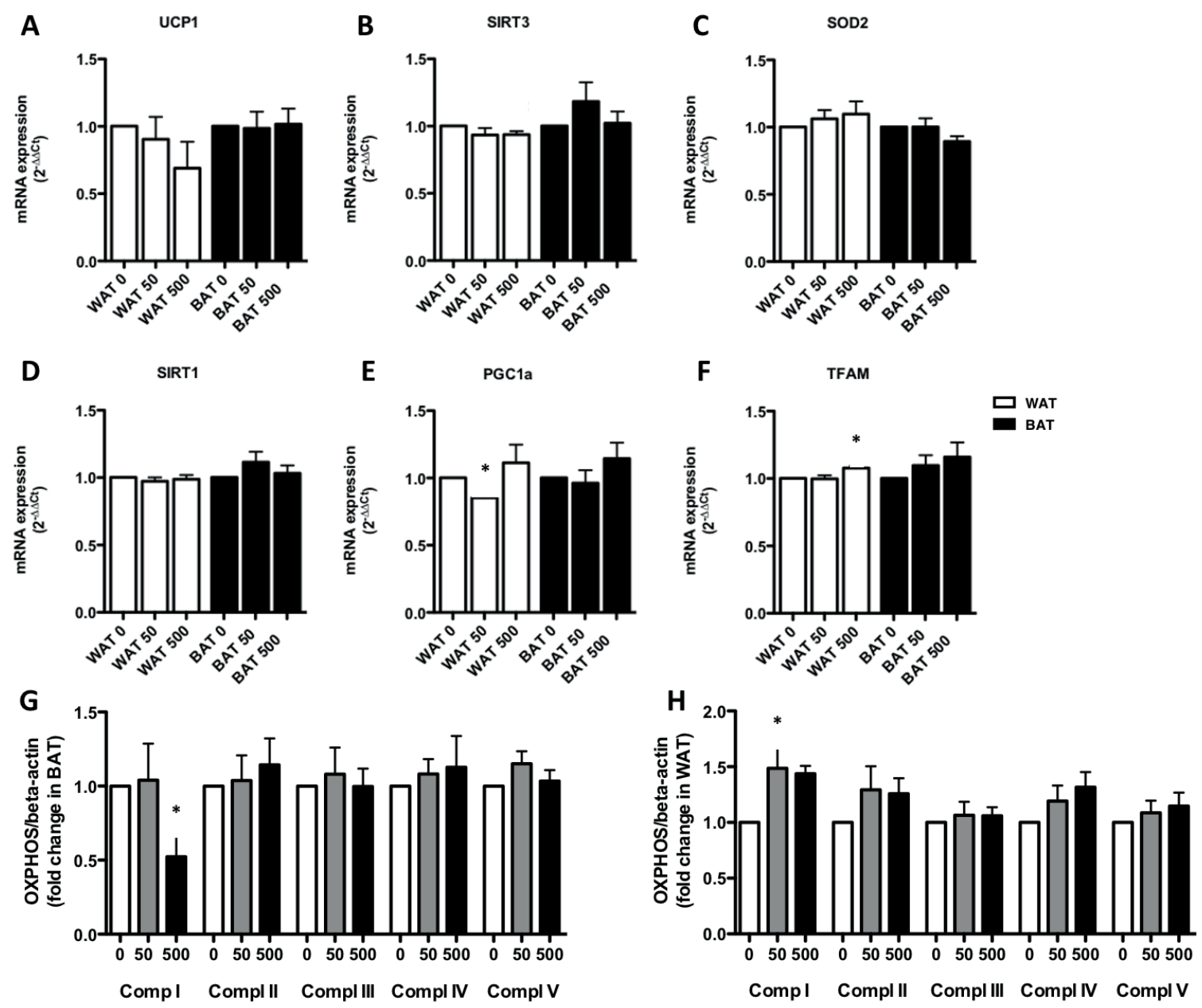

Figure 4. NR does not alter gene expression of SIRT targets in cultured primary adipocytes derived from human BAT. Cultured adipocytes derived from human BAT (black bars) and WAT (white bars) were stimulated for 24 hours with $0 \mu \mathrm{M}(0), 50 \mu \mathrm{M}(50)$ or $500 \mu \mathrm{M}(500) \mathrm{NR}$. Gene expression of UCP1 (A), SIRT3 (B), SOD2 (C), SIRT1 (D), PGC1a (E) and TFAM (F) were determined using qPCR techniques. Cultured adipocytes derived from human BAT (G) and WAT $(\mathrm{H})$ were stimulated for 24 hours with $0 \mu \mathrm{M}(0), 50 \mu \mathrm{M}(50)$ or $500 \mu \mathrm{M}(500) \mathrm{NR}$. OXPHOS complexes were analyzed by Western blot. Data is expressed as mean $\pm \mathrm{SE}$. ${ }^{*} \mathrm{p}<0.05$ compared to matching control $(n=4)$.

\section{NR does not affect BAT activity or energy expenditure in human volunteers}

Our results show that NR can enhance BAT activity in mice and human primary brown adipocytes. These promising results urged us to examine the true translational potential of NR on human BAT and therefore we performed a first double-blind placebo-controlled cross-over design clinical trial in a small cohort of obese volunteers. During six weeks, $1000 \mathrm{mg} /$ day NR or placebo was supplemented. Coldstimulated BAT activity was determined via uptake of [18F]-FDG using PET-CT and whole-body energy expenditure was also measured. However, NR supplementation for six weeks had no effect on [18F]-FDG mediated glucose uptake accessed as 
SUVmean and SUVmax in fixed volumes (Figure 5A and B). NR supplementation was also unable to increase cold-stimulated energy expenditure (Figure 5C and D). Also, non-shivering thermogenesis was unchanged following NR supplementation (Figure $5 \mathrm{E})$.

A

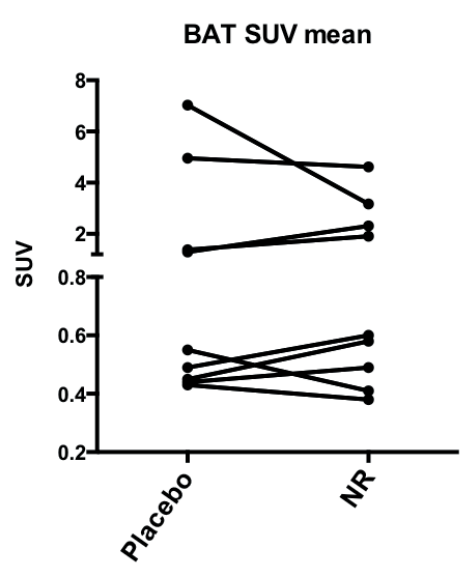

D

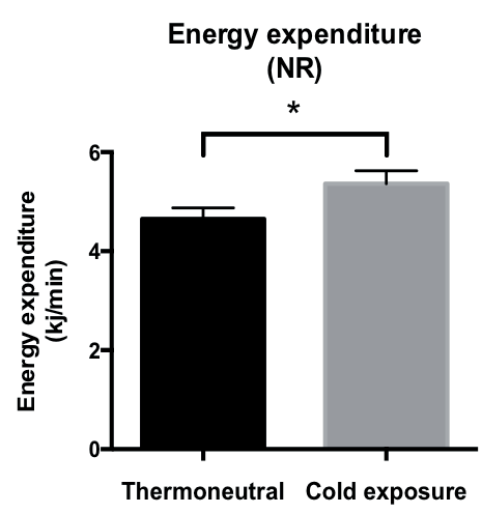

B

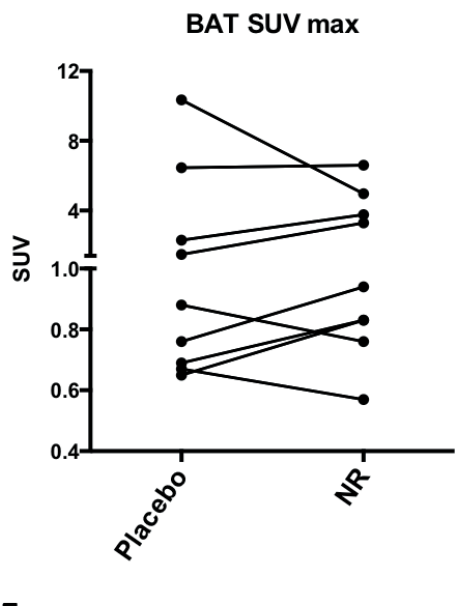

E
C

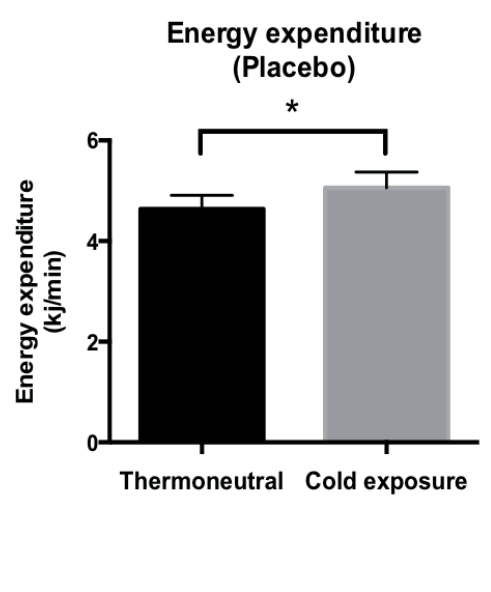

Figure 5. NR does not stimulate energy expenditure or BAT activity in humans. Human volunteers were supplemented with $1000 \mathrm{mg} /$ day NR for six weeks in a placebo-controlled cross over design. BAT SUV mean (A) and BAT SUV max in fixed volumes (B) following cold exposure. Energy expenditure at thermoneutrality and after cold exposure following placebo (C) or NR (D). Nonshivering thermogenesis (NST) following NR supplementation (E). Data is expressed as mean \pm SE. ${ }^{*} p<0.05(n=9)$ 


\section{DISCUSSION}

Preclinical data has demonstrated that increasing NAD+ levels can counteract harmful effects of metabolic disease, and NR supplementation is a powerful intervention to do so. Here, we specifically assessed the effect of NR, on mouse BAT in vivo and on adipocytes derived from human BAT and WAT in vitro and in vivo in human volunteers. In HFD-fed mice, NR supplementation resulted in enhanced tolerance to cold and decreased lipid droplet size. Similarly, in cultured adipocytes derived from human BAT, elevating NAD+ levels via NR enhanced NE-mediated mitochondrial uncoupling. This stimulatory effect was specific for adipocytes derived from human BAT, which is in line with earlier observation in animals where $\mathrm{NAD}^{+}$supplementation increased $\mathrm{NAD}^{+}$concentrations in BAT and stimulated BAT metabolism following a cold challenge (11). However, six weeks of supplementation with 1000 mg/day NR did not increase BAT activity in humans. These observations together show that NR and betaadrenergic signaling can work together to enhance BAT activity, however the current dose of NR in humans was unable to increase human BAT activity in vivo.

We examined lipolysis ex vivo in murine BAT and in vitro in human BAT in the context of NR stimulation. There are obvious differences when comparing murine adipose tissue explants to in vitro differentiated human adipocytes. However, in vitro lipolysis in adipocytes derived from human BAT was unaltered following NR and also ex vivo lipolysis in mouse BAT following NR exposure was unaffected. This indicates that the direct mode of action of NR is most likely independent of lipolysis in mice and humans. Previously, it has been reported that NR increases $\mathrm{NAD}^{+}$content in cells and mitochondria (11) and mitochondria are pivotal players in providing energy for lipolysis. In line with unaltered lipolysis in human and murine BAT, we also observed no change in OXPHOS protein, ATP levels or gene expression related to mitochondrial biogenesis in cultured adipocytes derived from human BAT. This was further substantiated with results from TOMM20 staining in mouse BAT which were unchanged following NR treatment. Also, the protein or mRNA abundance of the classical mitochondrial marker for BAT, UCP1, was unchanged following NR treatment in mouse BAT and in cultured adipocytes derived from human BAT, respectively. However, when it comes to UCP1 expression and NR, different results have been obtained depending on the experimental setup. In young lean mice on a chow diet supplemented for five weeks with NR, UCP1 protein content was increased in BAT, however, in this study total BAT mass was not reported (27). In another study, NR was administered directly after birth in mice which was subsequently followed by a HFD-challenge for ten weeks. Ucp1 mRNA expression was unaffected in animals on a HFD in BAT, but interestingly the animals on a control diet showed decreased 
expression of Ucp1 mRNA expression following NR in BAT (28). These findings together indicate that gene and/or protein expression alone might not be the best indicator for BAT metabolic activity.

Stimulating BAT activity by other means than cold in humans could have beneficial effects on whole body metabolism. Human BAT activity shows a negative correlation with obesity $(4,29)$, and stimulation of BAT activity through cold acclimatization increases energy expenditure that potentially could lead to loss of fat mass (30). Furthermore, cold-induced BAT activity is related to increased insulin sensitivity in humans $(31,32)$. Cold exposure is the most effective method to stimulate BAT activity; however, not the most practical one. Therefore, alternative routes to stimulate BAT in humans are warranted. To test if NR may have potential to activate BAT in humans, an in vitro model for human BAT activity is a good tool that can provide insights into human metabolism. Previously, we demonstrated that bile acid supplementation increased mitochondrial uncoupling in adipocytes derived from human BAT, a finding

we also confirmed in humans (8). Therefore, we believe that primary cultured adipocytes derived from human BAT are a good model to investigate compounds that activate human BAT. Furthermore, we have demonstrated that in vitro mitochondrial uncoupling in adipocytes derived from human BAT correlates with BMI and age, a finding that is also observed in human BAT activity in vivo(29). All these observations together show that an in vitro model can aid in providing information on in vivo metabolism of human BAT. In the current study, NR was able to stimulate BAT activity in human primary brown adipocytes. However, we could not confirm these results in a clinical trial in humans. The reason why NR had beneficial effects in human primary brown adipocytes but not in vivo in humans, cannot be deduced from the current study. Different dosage or duration of NR supplementation may be needed to exert beneficial effects of NR on human BAT in vivo. Therefore, further translational research is needed in order to better understand the differences in NAD+ metabolism between mouse and man. 


\section{ACKNOWLEDGEMENTS}

EBMN MM CR KG JAJ GS JA WDVML PS designed the study. EBMN MM CR KG JAJ GS performed experiments. EBMN MM CR KG JA WDVMK PS analyzed and interpreted the data. EBMN MM CR KG JA WDVMK PS contributed to the discussion and wrote, reviewed and edited the manuscript. All authors approve the last version of the manuscript. This research was funded by CVON ENERGISE grant CVON201402 to PS. JA is supported by École Polytechnique Fédérale de Lausanne (EPFL), the Velux Stiftung (1019), and the Swiss National Science Foundation (31003A_179435). The authors would like to thank Hongbo Zang for experimental assistance and reading the manuscript. PS is the guarantor of this work and, as such, had full access to all the data in the study and takes responsibility for the integrity of the data and the accuracy of the data analysis. The authors declare no conflict of interest relevant to this article. 


\section{REFERENCES}

1. Cannon B, Nedergaard J. Brown adipose tissue: function and physiological significance. Physiological reviews. 2004;84(1):277-359.

2. Cypess AM, Lehman S, Williams G, Tal I, Rodman D, Goldfine AB, et al. Identification and importance of brown adipose tissue in adult humans. The New England journal of medicine. 2009;360(15): 1509-17.

3. Saito M, Okamatsu-Ogura Y, Matsushita M, Watanabe K, Yoneshiro T, Nio-Kobayashi J, et al. High incidence of metabolically active brown adipose tissue in healthy adult humans: effects of cold exposure and adiposity. Diabetes. 2009;58(7):1526-31.

4. van Marken Lichtenbelt WD, Vanhommerig JW, Smulders NM, Drossaerts JM, Kemerink GJ, Bouvy ND, et al. Cold-activated brown adipose tissue in healthy men. The New England journal of medicine. 2009;360(15):1500-8.

5. Virtanen KA, Lidell ME, Orava J, Heglind M, Westergren R, Niemi T, et al. Functional brown adipose tissue in healthy adults. The New England journal of medicine. 2009;360(15):1518-25.

6. Orava J, Nuutila P, Lidell ME, Oikonen V, Noponen T, Viljanen T, et al. Different metabolic responses of human brown adipose tissue to activation by cold and insulin. Cell metabolism. 2011;14(2):272-9.

7. Watanabe M, Houten SM, Mataki C, Christoffolete MA, Kim BW, Sato H, et al. Bile acids induce energy expenditure by promoting intracellular thyroid hormone activation. Nature. 2006;439(7075):484-9.

8. Broeders EP, Nascimento EB, Havekes B, Brans B, Roumans KH, Tailleux A, et al. The Bile Acid Chenodeoxycholic Acid Increases Human Brown Adipose Tissue Activity. Cell metabolism. 2015;22(3):418-26.

9. Velazquez-Villegas LA, Perino A, Lemos V, Zietak M, Nomura M, Pols TWH, et al. TGR5 signalling promotes mitochondrial fission and beige remodelling of white adipose tissue. Nat Commun. 2018;9(1):245.

10. Cypess AM, Weiner LS, Roberts-Toler C, Franquet Elia E, Kessler SH, Kahn PA, et al. Activation of human brown adipose tissue by a beta3-adrenergic receptor agonist. Cell metabolism. 2015;21(1):33-8.

11. Canto C, Houtkooper RH, Pirinen E, Youn DY, Oosterveer MH, Cen Y, et al. The NAD(+) precursor nicotinamide riboside enhances oxidative metabolism and protects against high-fat diet-induced obesity. Cell metabolism. 2012;15(6):838-47.

12. Yang T, Chan NY, Sauve AA. Syntheses of nicotinamide riboside and derivatives: effective agents for increasing nicotinamide adenine dinucleotide concentrations in mammalian cells. Journal of medicinal chemistry. 2007;50(26):6458-61.

13. Trammell SA, Weidemann BJ, Chadda A, Yorek MS, Holmes A, Coppey LJ, et al. Nicotinamide Riboside Opposes Type 2 Diabetes and Neuropathy in Mice. Scientific reports. 2016;6:26933.

14. Vaur P, Brugg B, Mericskay M, Li Z, Schmidt MS, Vivien D, et al. Nicotinamide riboside, a form of vitamin B3, protects against excitotoxicity-induced axonal degeneration. FASEB journal : official publication of the Federation of American Societies for Experimental Biology. 2017.

15. Sorrentino V, Romani M, Mouchiroud L, Beck JS, Zhang H, D'Amico D, et al. Enhancing mitochondrial proteostasis reduces amyloid-beta proteotoxicity. Nature. 2017;552(7684):18793.

16. Zhang $H$, Ryu D, Wu $Y$, Gariani $K$, Wang $X$, Luan $P$, et al. NAD(+) repletion improves mitochondrial and stem cell function and enhances life span in mice. Science. 2016;352(6292):1436-43.

17. Ryu D, Zhang H, Ropelle ER, Sorrentino V, Mazala DA, Mouchiroud L, et al. NAD+ repletion improves muscle function in muscular dystrophy and counters global PARylation. Science translational medicine. 2016;8(361):361ra139.

18. Khan NA, Auranen M, Paetau I, Pirinen E, Euro L, Forsstrom S, et al. Effective treatment of mitochondrial myopathy by nicotinamide riboside, a vitamin B3. EMBO molecular medicine. 2014;6(6):721-31.

19. Gariani K, Menzies KJ, Ryu D, Wegner CJ, Wang X, Ropelle ER, et al. Eliciting the mitochondrial unfolded protein response by nicotinamide adenine dinucleotide repletion reverses fatty liver disease in mice. Hepatology. 2016;63(4):1190-204.

20. Lagouge M, Argmann C, Gerhart-Hines Z, Meziane H, Lerin C, Daussin F, et al. Resveratrol improves mitochondrial function and protects against metabolic disease by activating SIRT1 and PGC-1alpha. Cell. 2006;127(6):1109-22. 
21. Gnad T, Scheibler S, von Kugelgen I, Scheele C, Kilic A, Glode A, et al. Adenosine activates brown adipose tissue and recruits beige adipocytes via A2A receptors. Nature. 2014;516(7531):395-9.

22. Wu J, Bostrom P, Sparks LM, Ye L, Choi JH, Giang AH, et al. Beige adipocytes are a distinct type of thermogenic fat cell in mouse and human. Cell. 2012;150(2):366-76.

23. van der Lans AA, Hoeks J, Brans B, Vijgen GH, Visser MG, Vosselman MJ, et al. Cold acclimation recruits human brown fat and increases nonshivering thermogenesis. The Journal of clinical investigation. 2013;123(8):3395-403.

24. Vosselman MJ, Brans B, van der Lans AA, Wierts R, van Baak MA, Mottaghy FM, et al. Brown adipose tissue activity after a high-calorie meal in humans. The American journal of clinical nutrition. 2013;98(1):57-64.

25. Surwit RS, Kuhn CM, Cochrane C, McCubbin JA, Feinglos MN. Diet-induced type II diabetes in C57BL/6J mice. Diabetes. 1988;37(9):1163-7.

26. Houtkooper $\mathrm{RH}$, Pirinen E, Auwerx J. Sirtuins as regulators of metabolism and healthspan. Nature reviews Molecular cell biology. 2012;13(4):225-38.

27. Crisol BM, Veiga CB, Lenhare L, Braga RR, Silva VRR, da Silva ASR, et al. Nicotinamide riboside induces a thermogenic response in lean mice. Life sciences. 2018.

28. Serrano A, Asnani-Kishnani M, Rodriguez AM, Palou A, Ribot J, Bonet ML. Programming of the Beige Phenotype in White Adipose Tissue of Adult Mice by Mild Resveratrol and Nicotinamide Riboside Supplementations in Early Postnatal Life. Molecular nutrition \& food research. 2018:e1800463.

29. Nascimento EBM, Sparks LM, Divoux A, van Gisbergen MW, Broeders EPM, Jorgensen JA, et al. Genetic Markers of Brown Adipose Tissue Identity and In Vitro Brown Adipose Tissue Activity in Humans. Obesity. 2018;26(1):135-40.

30. Yoneshiro T, Aita S, Matsushita M, Kayahara T, Kameya T, Kawai Y, et al. Recruited brown adipose tissue as an antiobesity agent in humans. J Clin Invest. 2013;123(8):3404-8.

31. Hanssen MJ, van der Lans AA, Brans B, Hoeks J, Jardon KM, Schaart G, et al. Short-term Cold Acclimation Recruits Brown Adipose Tissue in Obese Humans. Diabetes. 2016;65(5):1179-89.

32. Iwen KA, Backhaus J, Cassens M, Waltl M, Hedesan OC, Merkel M, et al. Cold-Induced Brown Adipose Tissue Activity Alters Plasma Fatty Acids and Improves Glucose Metabolism in Men. The Journal of clinical endocrinology and metabolism. 2017;102(11):4226-34. 



\section{ABSTRACT}

Background \& Aims: Type 2 diabetes is a major health problem and is associated with increased cardiovascular disease risk. Cold exposure has received much attention over the past years as a potential strategy to improve metabolic health. We previously showed that ten days of cold acclimation with non-shivering thermogenesis (NST) markedly improved skeletal muscle insulin sensitivity in patients with type 2 diabetes. However, whether cold acclimation with NST also affects postprandial metabolism and cardiovascular risk markers remains unknown. Here, we aimed to investigate whether cold acclimation with NST beneficially affected postprandial metabolism and cardiovascular risk markers, and if the effect on insulin sensitivity was sustained for a longer period of time. In addition, we hypothesized that beneficial effects of cold exposure could be achieved by stimulating NST, hence paid special attention to the prevention of shivering in our participants.

Methods: Nine obese participants diagnosed with non-insulin dependent type 2 diabetes were exposed to mild cold $\left(16-17^{\circ} \mathrm{C}\right)$ for ten days. Before and immediately after the cold exposure postprandial glucose and lipid metabolism, vascular function measurements, insulin sensitivity and intrahepatic lipid content were determined. Furthermore, skeletal muscle biopsies were obtained. Lastly, insulin sensitivity was measured again ten days later after the last cold exposure session to assess the sustained effect of cold acclimation.

Results: Based on direct observations, questionnaire results and gene expression analyses in muscle biopsies, no shivering occurred during the ten-day cold acclimation. Ten-day cold acclimation with NST significantly increased postprandial energy expenditure (AUC pre: $2620 \pm 146$; post: $2752 \pm 168 \mathrm{~kJ}, \mathrm{p}=0.03$ ). In addition, overnight fasted carbohydrate oxidation was significantly increased (pre $3.76 \pm 0.47$ vs. post $4.97 \pm 0.68 \mu \mathrm{mol} / \mathrm{kg} / \mathrm{min}, \mathrm{p}<0.01)$ and fat oxidation significantly decreased after the cold acclimation (pre $3.80 \pm 0.17 \mathrm{vs}$. post $3.57 \pm 0.17 \mu \mathrm{mol} / \mathrm{kg} / \mathrm{min}, \mathrm{p}<0.01$ ). Aortic augmentation index was significantly improved after cold acclimation measured in the overnight fasted state (pre $22.57 \pm 1.36 \%$ vs. post $19.84 \pm 1.96 \%$, $\mathrm{p}=0.03)$. Cold acclimation with NST had no effect on postprandial glucose and lipid metabolism, intrahepatic lipid content or other vascular function markers. Surprisingly, insulin sensitivity was unaffected both when measured directly (one day) or ten days after the last cold exposure.

Conclusion: A ten-day cold acclimation period at $16-17{ }^{\circ} \mathrm{C}$ under non-shivering conditions resulted in mild effects on substrate oxidation and cardiovascular risk markers. The lack of marked effects in the current study may be attributed to the absence of shivering compared to previous studies. This suggests that shivering is an essential component of the metabolic health effects induced by cold exposure. 


\section{INTRODUCTION}

Mortality rates in type 2 diabetes mellitus (T2DM) patients are approximately twice as high compared to individuals without T2DM and can mostly be attributed to an increased risk of coronary heart diseases $(1,2)$. In the last decade, cold exposure as a tool to alleviate insulin resistance has attracted lots of scientific interest. Currently, there is little demand for the human body to adjust to colder temperatures, since humans spend most of their time in a well-controlled indoor environment with optimal temperatures within the body's thermoneutral zone. The physiological reaction of the human body to cold exposure includes simultaneously 1) insulative responses by peripheral vasoconstriction and 2) an increase in metabolic rate by shivering thermogenesis (ST) and/or non-shivering thermogenesis (NST). It has been shown that during daily cold exposure in humans, shivering gradually decreases within 1020 days, while the related increase in metabolic rate remains at a stable level (3-5). This indicates that acclimation to cold occurs and that ST can be replaced by NST. Both brown adipose tissue (BAT) (6-9) and skeletal muscle $(4,10)$ have been identified as contributors to NST.

Cold acclimation has been shown to promote insulin sensitivity in humans, originally attributed to increased BAT activity $(11,12)$. Research performed within our group, however, has shown that ten days of cold acclimation $\left(14-15^{\circ} \mathrm{C}\right)$ markedly improved skeletal muscle insulin sensitivity by $43 \%$ in patients with type 2 diabetes (13), an improvement that is comparable to the effect seen after long term exercise training (14). Interestingly, the cold induced improvement of insulin sensitivity did not appear to originate form BAT activation but was associated with increased GLUT4 translocation in skeletal muscle (13). Therefore, these findings indicate that effects of cold acclimation on skeletal muscle may be a novel approach to improve metabolic health in patients with T2DM.

Hyperglycemia and dyslipidemia are common metabolic abnormalities in patients with T2DM (15), that most commonly are alleviated upon insulin sensitizing interventions. Interestingly, animal studies have shown reduced postprandial lipids upon prolonged cold exposure (16). Moreover, a postprandial reduction in hypertriglyceridemia in human individuals was associated with BAT (17). Lowering postprandial hyperglycemia and hypertriglyceridemia is clinically relevant as high plasma glucose and triglyceride levels can cause damage to the vascular wall inducing an impaired vascular function (18), which causes atherosclerotic plaque development that may ultimately lead cardiovascular disease events. However, the 


\section{Chapter 4}

effect of cold exposure on these cardiovascular risk markers has not yet been investigated in humans.

Therefore, we primarily aimed to investigate the effect of ten days cold acclimation with NST in overweight and obese patients with T2DM on postprandial glucose and lipid metabolism and cardiovascular risk markers. Secondly, we investigated whether a ten-day cold acclimation period with NST affects insulin sensitivity, and if so, if this effect is sustained for another ten days at room temperature. Given the previous results on skeletal muscle (13), we also aimed to test the hypothesis that beneficial effects of cold exposure can be truly achieved by stimulating NST, and hence took specific care to prevent shivering in our participants.

\section{METHODS}

\section{Participants}

Ten obese men and women were included in the study with one volunteer dropping out because of personal reasons. Hence, nine participants completed the study. All participants underwent a screening including assessment of blood parameters, electrocardiography, anthropometric measurements and a questionnaire to evaluate eligibility. Inclusion criteria were: $45-70$ years of age, BMI $27-35 \mathrm{~kg} / \mathrm{m}^{2}$, diagnosed with type 2 diabetes for at least one year (relatively well-controlled $\mathrm{HbA} 1 \mathrm{c}<8.5 \%$ ), use of oral glucose lowering drugs (metformin and/or sulfonylurea agents), a sedentary lifestyle (<3 h exercise per week), non-smoking for at least six months, no alcohol use of $>2$ servings per day and a stable body weight for at least six months. Other medication without known effects on the primary outcome measurement was allowed. Data were collected between March 2016 and August 2017.

\section{Ethical approval}

The study was conducted according to the declaration of Helsinki and was approved by the Ethics Committee of the Maastricht University Medical Center. The study was registered at https://trialregister.nl (NL4469/NTR5711). All participants provided their written informed consent before screening.

\section{Study design}

Figure 1 provides an overview of the study design. A ten-day cold acclimation intervention was performed essentially as previously described (13), although the environmental temperature was set approximately $1-2^{\circ} \mathrm{C}$ higher in the current study, 
at $16-17^{\circ} \mathrm{C}$, to avoid shivering. Before the onset of the cold acclimation period a highfat meal test (meal test pre) was combined with several vascular function measurements. Three days later, intrahepatic lipid content was measured followed by a muscle biopsy and a hyperinsulinemic-euglycemic 2-step clamp (clamp pre). Subsequently, a ten-day cold acclimation intervention started (Day 1-10). One day after the last cold exposure, (Day 11), the first test day was repeated (meal test post). The following two days (Day 12-13), additional cold exposure was maintained in order to sustain the intervention effect for the re-tests on Day 14 (clamp post). Ten days after termination of the cold acclimation, i.e. without further intervention, another muscle biopsy was taken followed by a hyperinsulinemic-euglycemic 2-step clamp (clamp long term) to assess the potential long-term effect of cold acclimation on whole body insulin sensitivity. Two months after the last test day, fasting blood samples were taken to measure markers for glucose homeostasis. All tests were executed at room temperature. In the evening preceding all test days, participants consumed a standardized meal and remained fasted from 20:00 h onwards. In addition, participants were asked to refrain for at least 48 hours from any physical activity different from their daily routine. Participants arrived at the testing facility by car. Blood glucose lowering medication was discontinued on the morning of the clamps.

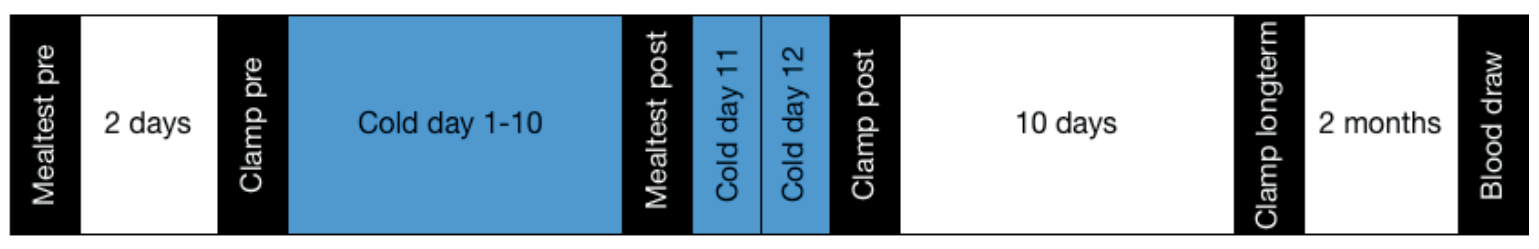

Figure 1. Study design

A meal test and clamp were performed before cold acclimation, separated by two days. After ten days of cold exposure a second meal test was performed, followed by two additional days of cold exposure and followed by a second clamp. Ten days after the last intervention day, a third clamp was performed. Two months after the last clamp another blood sample was collected. Blue boxes represent cold acclimation intervention $\left(16-17^{\circ} \mathrm{C}\right)$. White boxes represent no intervention. Black boxes represent test days.

\section{Cold acclimation}

During cold acclimation, participants were progressively exposed to an environmental temperature of $16-17^{\circ} \mathrm{C}$ for ten consecutive days: two hours on day 1 , four hours on day 2 and six hours on days 3 to 10. On Day 11-12, after the first post-intervention measurements, participants were again cold exposed for six hours. Participants were dressed in short-sleeved T-shirts and shorts and remained sedentary while staying in the cold room and were instructed to not change their habitual diet during the entire 
study period. Food intake while staying in the cold room was kept constant. Wireless temperature sensors (iButtons, Maxim Integrated, San Jose, CA, USA) were placed on 15 ISO-defined sites on days 3 and 10 of the cold acclimation period to measure skin temperature. Average skin temperature as well as proximal and distal temperature was calculated as described before (19). Participants were not allowed to shiver. In case shivering started, extra clothing was immediately provided to ensure that shivering stopped. At selected timepoints during day 3 and 10 VAS scales on thermal sensation, thermal comfort and shivering were completed. Incremental AUCs (iAUC) were calculated to determine subjective responses during the cold acclimation period, as described before (8).

\section{High-fat meal test}

After placing an intravenous cannula, a fasted blood sample was drawn $(T=0)$ and energy expenditure and substrate oxidation was measured via indirect calorimetry. At 09:00 h, participants were asked to consume a high-fat shake, within ten minutes. The nutritional content of the shake is shown in Supplementary Table 2. Subsequently, blood samples were drawn after 15, 30, 45, 60, 90, 120, 180, 240, 300, 360, 420 and 480 minutes. After the blood sampling at $T=240$, at 13:00 h, the participants consumed a second high-fat shake with the exact same contents as in the morning to investigate the second-meal effect, as described before (20). At T30-60, T90-120, T210-240, T270-300, T350-380 and T450-480 indirect calorimetry was performed to measure energy expenditure and substrate oxidation. Participants were not allowed to eat or drink anything else throughout the meal test, except for water.

\section{Vascular function measurements}

On the morning of the meal tests, radial artery pulse wave analysis (PWA) was performed with a tonometer (SphygmoCor v9, AtCor Medical, West Ryde, Australia), from which the aortic augmentation index corrected for heart rate (AlxHR75) was calculated as described before (21). Using the same tonometer, carotid-to-femoral pulse wave velocity $\left(P W V_{c-f}\right)$ was also determined as described before (21). These vascular stiffness measurements were performed before (T0) and during the high-fat meal tests at two different time points (T120 and T300).

Retinal vascular images were obtained to measure microvascular effects of the cold acclimation. Retinal images were obtained as described before using a nonmydriatic retinal camera (Topcon TRC-NW-300; Topcon Co.) (22). At least two arteriolar and two venular segments were measured and summarized by using the Parr-Hubbard formulas (23), special care was taken to examine the exact same segments before and after the cold acclimation period. 


\section{Hyperinsulinemic-euglycemic clamp}

To determine insulin sensitivity, a two-step hyperinsulinemic euglycemic clamp (24) with co-infusion of D-[6.6- ${ }^{2} \mathrm{H}_{2}$ ] glucose tracer $(0.04 \mathrm{mg} / \mathrm{kg} / \mathrm{min})$ was performed, as described before (25). In short, insulin-suppressed endogenous glucose production (EGP) during low insulin infusion $\left(10 \mathrm{mU} / \mathrm{m}^{2} / \mathrm{min}\right)$ was measured as a reflection of hepatic insulin sensitivity. This was followed by a high insulin infusion $\left(40 \mathrm{mU} / \mathrm{m}^{2} / \mathrm{min}\right)$ to measure whole body glucose disposal (Rd). Indirect calorimetry was performed during baseline, low insulin and high insulin to measure energy expenditure and substrate oxidation. Steele's single pool non-steady state equations were used to calculate glucose appearance (Ra) and glucose disposal (Rd) (26). Volume of distribution was assumed to be $0.160 \mathrm{l} / \mathrm{kg}$ for glucose. Whole-body insulin sensitivity $\left(\mathrm{S}_{\mathrm{i}}\right)$ was calculated according to Bergman et al. (27), taking differences in insulin and glucose levels into account: $S_{i}=\Delta R d /\left(\Delta\right.$ insulin ${ }^{*}$ clamping glucose), where $\Delta$ represents the change from the basal state to the insulin-stimulated condition. EGP was calculated as Ra minus exogenous glucose infusion rate. Non-oxidative glucose disposal (NOGD) was calculated as Rd minus carbohydrate oxidation.

\section{Indirect calorimetry}

Whole body oxygen consumption and carbon dioxide production were measured during specific timepoints in the meal tests and the clamps using an automated respiratory gas analyzer with a ventilated hood system (Omnical; Maastricht Instruments, Maastricht, The Netherlands). Participants were measured in supine position for 30 minutes each time. Energy expenditure, glucose oxidation and fat oxidation rates were calculated using equations based on the measured averaged oxygen and carbon dioxide concentrations with the assumption that protein oxidation was negligible $(28,29)$.

\section{Intrahepatic lipid quantification by MR spectroscopy}

Proton magnetic resonance spectroscopy ( $\left.{ }^{1} \mathrm{H}-\mathrm{MRS}\right)$ was used to quantify intrahepatic lipid content $(\mathrm{IHL})$ at 07:00 $\mathrm{h}$ in the morning of Clamp pre and Clamp post. All measurements were performed on a 3.0T whole body scanner (Achieva Tx, Philips Healthcare, Best, The Netherlands). Spectra were acquired as described before (30). Values are given as $\mathrm{T} 2$ corrected ratios of the $\mathrm{CH}_{2}$ peak relative to the unsuppressed water peak, expressed as percentage.

\section{Skeletal muscle biopsies}

On each clamp day, a muscle biopsy was taken from the $\mathrm{m}$. vastus lateralis under local anesthesia (2\% lidocaine, without epinephrine) using the Bergström technique (31). The muscle biopsy was taken before the start of the insulin infusion on the 
morning of the clamp, at 08:30 h after an overnight fast. The biopsy immediately frozen in melting isopentane cooled with liquid nitrogen for biochemical analyses and immunohistochemical analyses.

\section{Histochemical analysis of GLUT4 in skeletal muscle biopsies}

Muscle biopsies taken in the overnight fasted state, prior to all three clamps, were analyzed for GLUT4 translocation. Sections were stained for GLUT4 as described previously (13), with primary antibodies against GLUT4 (sc-1608, Santa Cruz, Dallas, USA) and Laminin (L-9393, Sigma, St. Louis, USA), and appropriate conjugated secondary antibodies AlexaFluor488 (AF488, Invitrogen, Life Technologies Europe, Bleiswijk, The Netherlands) and AlexaFluor555 (AF555, Invitrogen, Life Technologies Europe). Images were acquired with a Nikon E800 fluorescent microscopy (Nikon, Amsterdam, The Netherlands) as described previously (13). Images were analyzed using ImageJ (NIH, Bethesda, USA) (32). Mean intensity of GLUT4 was measured on the cell membranes and in the cytosol.

\section{Shivering markers in skeletal muscle biopsies}

RNA extraction and cDNA synthesis from skeletal muscle was performed as previously described $(33,34)$. In short, RNA was isolated from skeletal muscle biopsies using Trizol followed by purification using the RNeasy kit from Qiagen (Hildenberg, Germany). cDNA was created by using the high-capacity RNA-to-cDNA kit from Applied Biosystems (Foster City, USA). mRNA expression was determined using a CFX384 Touch Real-Time PCR Detection System from BioRad Laboratories (Hercules, CA) using the following Taqman assays: CHRNA1 (Hs00909664), CCDC39 (Hs00977326), (MYH8 (Hs00267293), CCDC80 (Hs00277341), CHRND (Hs00897937), LYRM9 (Hs00953770), ACTC1 (Hs01109515) and RRAD (Hs00188163). Gene expression was normalized to RPLPO (fwd: CCATTCTATCATCAACGGGTACAA, rev: AGCAAGTGGGAAGGTGTAATCC) and expression was analyzed via the standard curve method.

\section{Blood sampling and analyses}

Blood collected in EDTA-coated tubes was immediately stored on ice, centrifuged and plasma was stored at $-80{ }^{\circ} \mathrm{C}$ until further analyses. Blood collected in serumtubes was stored at room temperature for at least 30 minutes to allow coagulation, followed by centrifugation and storage at $-80{ }^{\circ} \mathrm{C}$ until further analyses. Glucose (HkCP, Axonlab, Amsterdam, The Netherlands) and FFA (NEFA-HR, WAKO chemicals, Neuss, Germany) were determined enzymatically in EDTA plasma derived from the meal test samples using a Pentra 400 (Horiba, Montpellier, France). Insulin levels 
during the meal test were analyzed using RIA, and insulin levels during the clamp were analyzed using ELISA. Triglycerides (Sigma, Zwijndrecht, The Netherlands), cholesterol (CHOD-PAP, Roche Diagnostics, Mannheim, Germany) and HDLcholesterol (CHOD-PAP, Roche Diagnostics, Mannheim,Germany) after precipitation of apoB-containing lipoproteins with phosphotungstic acid and magnesium ions, were analyzed in serum also using a Pentra 400. LDL-cholesterol was calculated according the Friedewald equation (35).

\section{Statistical analyses}

Participant characteristics are reported as mean \pm SD. Other results are reported as mean \pm SE. Data are presented for $n=9$, unless otherwise indicated. Differences between Clamp pre, Clamp post and Clamp longterm were analyzed with a nonparametric Friedman test. Posthoc analyses were performed with Dunn's multiple comparison test. Differences between Meal test pre and Meal test post were analyzed with a Wilcoxon matched pairs signed rank test. Differences between muscle gene expression in the current and previous study were analyzed with a Mann-Whitney test. Statistical significance was set at $p<0.05$. Statistical analyses were performed using IBM SPSS version 23.0 for MacOSx (IBM, Armonk, NY, USA).

\section{RESULTS}

\section{Participant characteristics}

Nine overweight to obese men and women (age $65 \pm 5$ years; BMI $32.1 \pm 2.8 \mathrm{~kg} / \mathrm{m}^{2}$; four women) participated in the study (see Table 1). As defined by the inclusion criteria, participants were diagnosed for at least one year with type 2 diabetes and were treated with oral medication only (see Supplementary Table 1). Participants were non-smokers, had no other active diseases and had a sedentary lifestyle according to the Baecke questionnaire score $(7.94 \pm 2.00$, Table 1$)$.

Body and room temperature, thermal comfort and shivering during cold acclimation Room temperature during cold acclimation was on average $16.4 \pm 0.30^{\circ} \mathrm{C}$, which was approximately $1.4^{\circ} \mathrm{C}$ higher compared to the previous study by Hanssen et al (13). Average skin temperature dropped from $27.5 \pm 0.33^{\circ} \mathrm{C}$ to $26.3 \pm 0.97{ }^{\circ} \mathrm{C}$ during day 3. This was similar to the drop in average skin temperature from $27.4 \pm 0.80^{\circ} \mathrm{C}$ to $26.4 \pm 1.26{ }^{\circ} \mathrm{C}$ on day 10 . Thermal sensation and thermal comfort, assessed via VAS scales, was not significantly different between day 3 and day 10 of cold acclimation, as shown in Supplementary Figure 1. The temperature was progressively perceived colder and more uncomfortable over time. The shivering questionnaires 


\section{Chapter 4}

revealed that participants experienced no shivering and only occasionally reported tense muscles. Self-reported shivering intensity was less than described in the study from Hanssen et al (13) and were not significant different between day 3 and day 10 of cold acclimation (Figure 6A).

Table 1. Participant characteristics

\begin{tabular}{ll}
\hline Parameter & Mean \pm SD \\
\hline Gender F/M & $4 / 5$ \\
Age (years) & $65 \pm 5$ \\
Body weight $(\mathrm{kg})$ & $93.7 \pm 17.3$ \\
Height $(\mathrm{m})$ & $1.70 \pm 0.10$ \\
BMI $\left(\mathrm{kg} / \mathrm{m}^{2}\right)$ & $32.1 \pm 2.8$ \\
HbA1c $(\%)$ & $7.3 \pm 0.7$ \\
TG $(\mathrm{mmol} / \mathrm{L})$ & $1.54 \pm 0.34$ \\
ASAT $(\mathrm{U} / \mathrm{L})$ & $24 \pm 8$ \\
ALAT (U/L) & $37 \pm 19$ \\
GGT (U/L) & $34 \pm 13$ \\
eGFR (ml/min/1.73mm²) & $79 \pm 9$ \\
Physical activity level (Baecke score) & $7.51 \pm 1.16$ \\
Abbreviations: BMI, body mass index; HbA1C, hemoglobin A1C; TG, triglycerides; ASAT, aspartate \\
aminotransferase; ALAT, alanine aminotransferase; GGT, gamma-glutamyl transferase; eGFR, \\
estimated glomerular filtration rate according CKD-EPI method.
\end{tabular}

\section{Postprandial metabolism and substrate kinetics}

Meal test total area-under-the-curve values (AUC) for glucose, insulin and triglycerides were not significantly different before and after cold acclimation $(p=0.43$, $p=0.65$ and $p=0.50$ respectively, Figure $2 \mathrm{~A}-\mathrm{C}$ and Supplementary Table 4). In addition, no significant differences were observed in AUC when the $1^{\text {st }}$ or $2^{\text {nd }}$ meal of the meal test were analyzed separately (Supplementary Table 4). Area under the curve for plasma free fatty acids was also not significantly different before and after ten days of cold acclimation ( $p=0.16$, Figure $2 D$ and Supplementary Table 4). However, when the $1^{\text {st }}$ and $2^{\text {nd }}$ meal of the meal test were analyzed separately, the total AUC for FFA during the $1^{\text {st }}$ meal was significantly lower after cold acclimation (pre: $94228 \pm 5115 \mathrm{mmol} / \mathrm{l}$; post: $86014 \pm 4545 \mathrm{mmol} / \mathrm{l}, \mathrm{p}=0.039$ ) as shown in Supplementary Table 4.

Total energy expenditure during the meal tests, calculated as AUC, was significantly higher after the cold acclimation (pre: $2620 \pm 146$; post: $2752 \pm 168 \mathrm{~kJ}, p=0.03$, Figure $3 \mathrm{~A}$ and Supplementary Table 4). Although this elevated energy expenditure seemed 
to be mainly due to higher glucose oxidation, the differences in glucose and fat oxidation during the meal tests were not statistically significant $(p=0.44$ and $p>0.99$ respectively, Figure 3B-C, Supplementary Table 4).
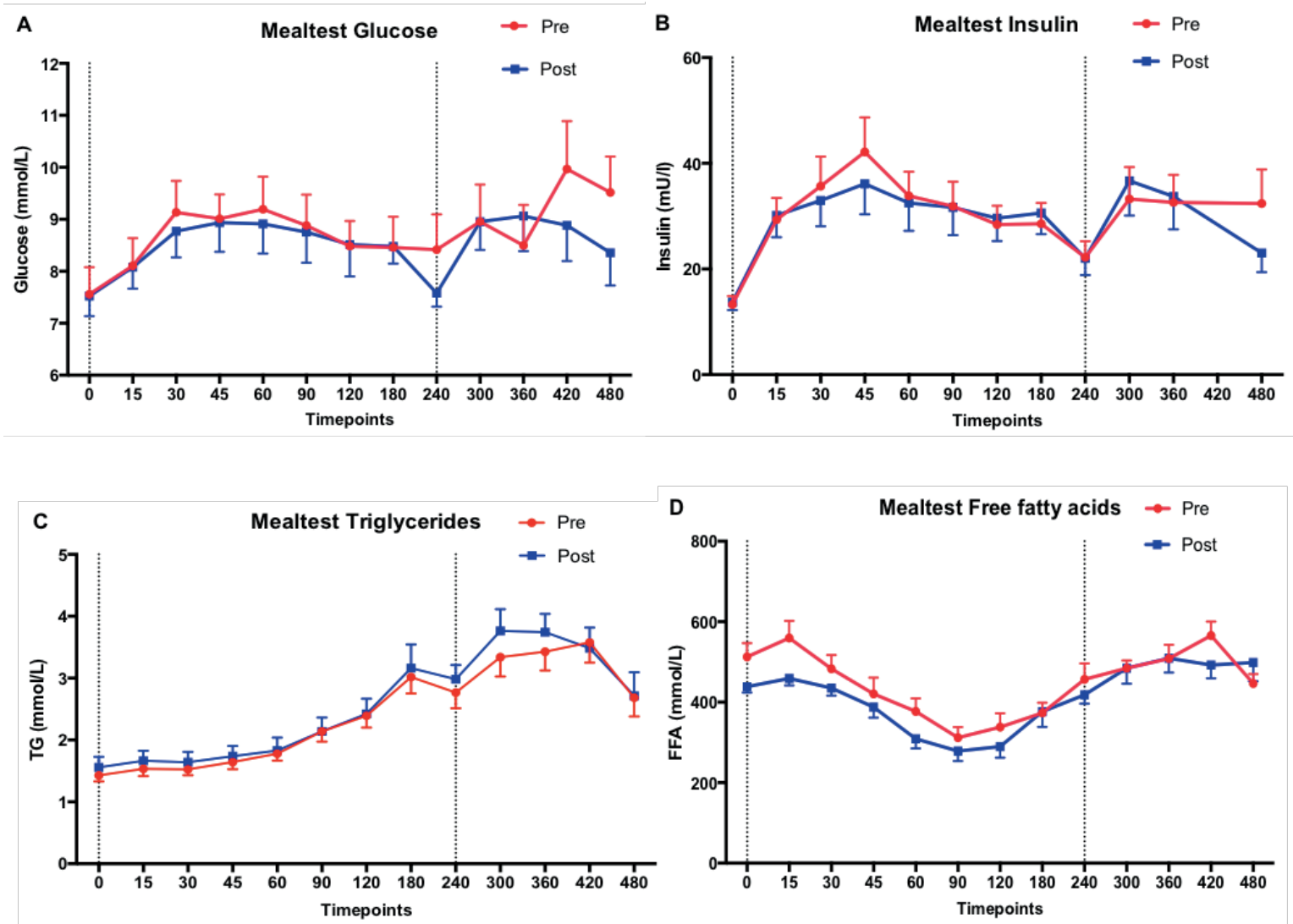

Figure 2. Plasma levels during the meal tests

A: plasma levels of glucose presented, B: Insulin, C: Triglycerides and D: Free fatty acids during the meal test. Pre cold acclimation is presented as the red line, post cold acclimation as the blue line. Dashed vertical lines indicate the time of consumption of the 1st shake at T0 and the 2nd shake at T240. $n=9$. Data is presented as mean \pm SE.

The results of the meal test suggest that cold acclimation may increase energy expenditure, probably fueled by higher carbohydrate oxidation. To further test this, we analyzed energy expenditure and substrate oxidation measured in the morning of the clamps. Overnight fasted energy expenditure at the start of the clamp (baseline) was not affected by cold acclimation (Supplementary Table 3). However, energy expenditure during the high insulin phase was significantly higher after cold acclimation compared to before (pre $4.67 \pm 0.30 \mathrm{vs}$. post $4.89 \pm 0.27 \mathrm{~kJ} / \mathrm{min}, p=0.03$ ) and this elevation tended to return to pre-intervention levels on the longterm (post $4.89 \pm 0.27$ vs. Iong term $4.67 \pm 0.18 \mathrm{~kJ} / \mathrm{min}, p=0.10$, Supplementary Table 3 ) Carbohydrate oxidation after an overnight fast was significantly higher after ten days 
of cold acclimation compared to before (pre $3.76 \pm 0.47$ vs. post $4.97 \pm 0.68$ $\mu \mathrm{mol} / \mathrm{kg} / \mathrm{min}, \mathrm{p}<0.01$, Supplementary Table 3$)$. In addition, fat oxidation after an overnight fast was significantly lower directly after cold acclimation compared to before (pre $3.80 \pm 0.17$ vs. post $3.57 \pm 0.17 \mu \mathrm{mol} / \mathrm{kg} / \mathrm{min}, \mathrm{p}<0.01$, Supplementary Table 3). These effects on carbohydrate and fat oxidation did not sustain on the long term (Supplementary Table 3). The changes in substrate oxidation after cold acclimation were not observed during insulin infusion (Supplementary Table 3).

There was no significant difference after cold acclimation for baseline HDL (pre 1.12 \pm 0.12 vs. post $1.21 \pm 0.08 \mathrm{mmol} / \mathrm{l}, \mathrm{p}=0.16$ ), LDL (pre $1.80 \pm 0.24 \mathrm{vs}$. post $1.84 \pm 0.24$ $\mathrm{mmol} / \mathrm{l}, \mathrm{p}=0.82$ ) or total cholesterol (pre $3.57 \pm 0.31 \mathrm{vs}$. post $3.76 \pm 0.31 \mathrm{mmol} / \mathrm{l}$, $p=0.16)$.

A
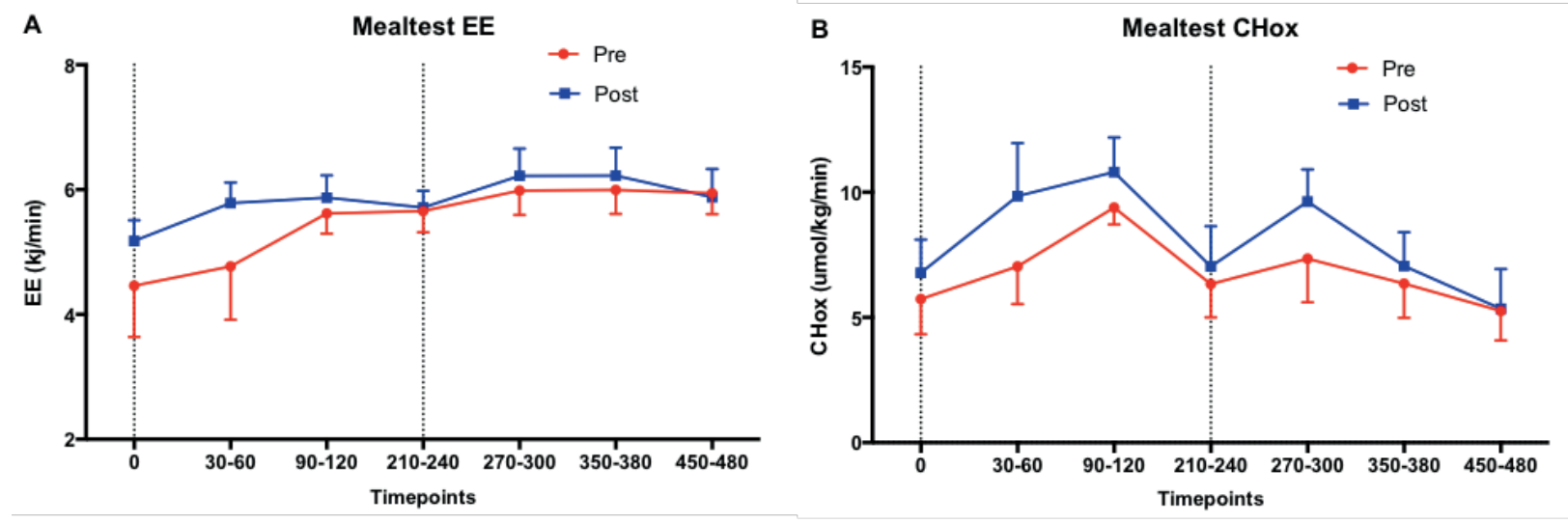

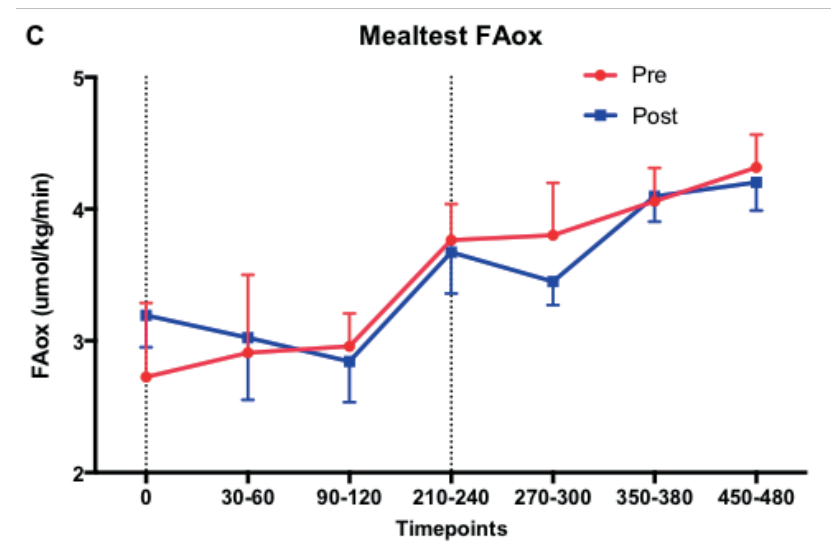

Figure 3. Energy expenditure and substrate oxidation during the meal tests A: Energy expenditure, B: Carbohydrate oxidation (CHox), and C: Fatty acid oxidation (FAox) during the meal test. Pre cold acclimation is presented as the red line, post cold acclimation as the blue line. Dashed vertical lines indicate the time of consumption of the 1st shake at T0 and the 2 nd shake at T240. $n=7$. Data is presented as mean \pm SE. 


\section{Vascular function}

AlxHR75, an indirect marker of arterial stiffness, was significantly improved after ten days of cold acclimation measured in the overnight fasted state (pre T0 $22.57 \pm 1.36$ $\%$ vs. post T0 $19.84 \pm 1.96 \%, p=0.03$, Figure 4A). Before cold acclimation, AlxHR75 tended to decrease upon the first meal ingestion (T120) and this meal-induced effect became significant after the second meal (T300). No statistically significant mealeffects on AlxHR75 were observed after cold acclimation (pre T120 $15.63 \pm 2.80 \%$ vs. post T120 $18.26 \pm 1.67 \% p=0.50$ and pre T300 $14.94 \pm 2.55 \%$ vs. post T300 $16.33 \pm 2.70 \% p=0.82$, Figure 4A). Postprandial changes in AlxHR75 were also not significant different before and after cold acclimation (pre delta $120-6.94 \pm 2.31$ vs. post delta $120-1.58 \pm 1.96, p=0.16$, pre delta $300-7.63 \pm 1.83$ vs. post delta $300-$ $3.51 \pm 1.91, p=0.30$, Figure 4B).

A

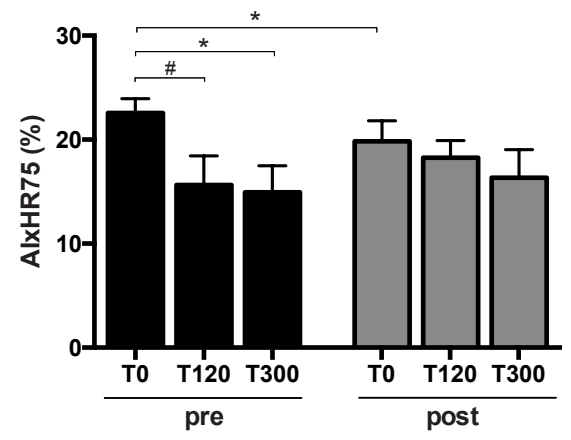

C

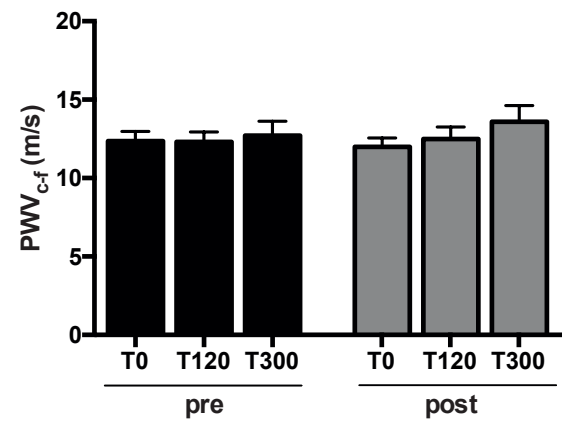

B

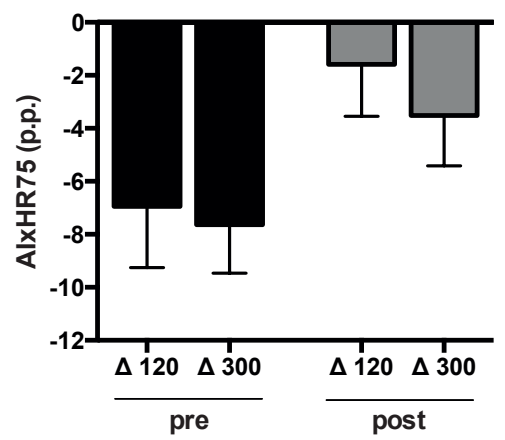

D

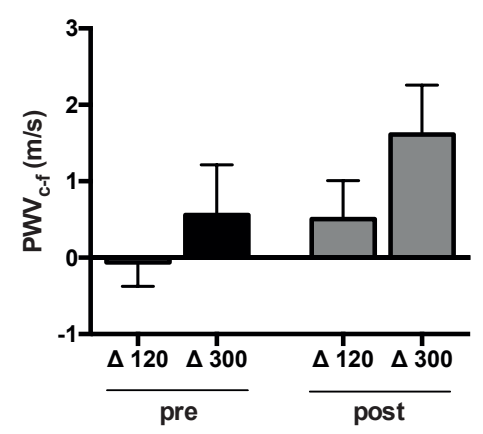

E

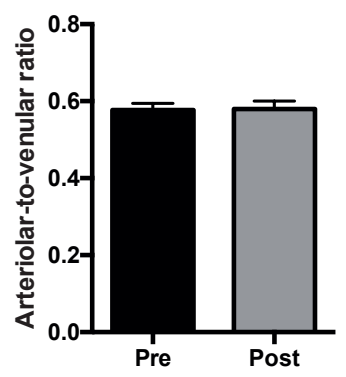

Figure 4. Vascular function markers

A: Aortic augmentation index (AlxHR75) at timepoints T0, T120, T300. B: AlxHR75 delta's T0-120 and T0-300 expressed as percentage points. C: Pulse wave velocity (PWVC-f) at timepoints T0, T120, T300 ( $n=8$ at T300). D: PWVc-f delta's T0-120 and T0-300 ( $n=8$ at delta T0-300). E: Arteriolar-to-venular ratio of the retinal vessels in the right eye $(n=8)$. Black bars represent data from the meal test before cold acclimation, grey bars represent data from the meal test after cold acclimation. Data shown are shown as mean \pm SE. ${ }^{*} p<0.05, \# p<0.10$. 
The current non-invasive gold standard technique to measure arterial stiffness ( $P W V_{c-}$ f), was however not affected by cold acclimation in the overnight fasted state $\mathrm{PWV}_{\mathrm{c}-\mathrm{f}}$ (pre T0 $12.36 \pm 0.61$ vs. post T0 $11.99 \pm 0.57, p=0.36$, Figure 4C). As expected, no meal-induced effects were observed (see Figure 4D). Finally, ten days of cold acclimation did not significantly affect fasting retinal vessel diameter, as the arteriolar width (pre $120.83 \pm 7.48 \mu \mathrm{m}$ vs. post $120.52 \pm 7.89 \mu \mathrm{m}, \mathrm{p}=0.95$ ), venular width (pre $209.19 \pm 10.73 \mu \mathrm{m}$ vs. post $207.17 \pm 10.07 \mu \mathrm{m}, \mathrm{p}=0.23)$ and the arteriolar-to-venular ratio (pre $0.58 \pm 0.02$ vs. post $0.58 \pm 0.02, p=0.84$, Figure $4 \mathrm{E}$ ) did not change.

\section{Intrahepatic lipid content}

As we hypothesized that cold acclimation could affect postprandial lipid metabolism, we also investigated if cold acclimation affects intrahepatic lipid content. However, intrahepatic lipid content was not different before and directly after cold acclimation (pre $6.1 \pm 4.3$ vs. post $7.0 \pm 4.0 \%, p=0.22, n=8$, Figure 5D).

A

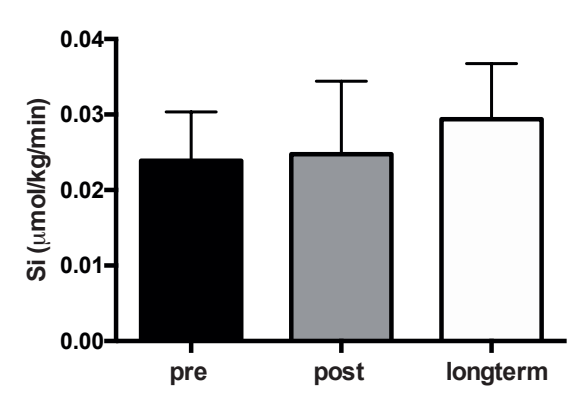

C

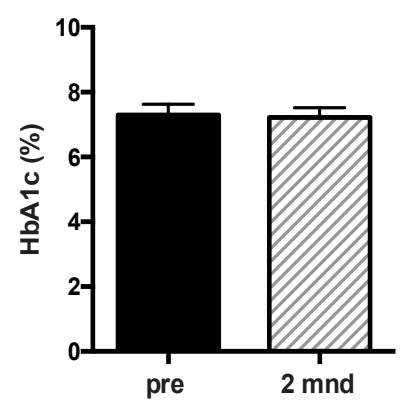

B

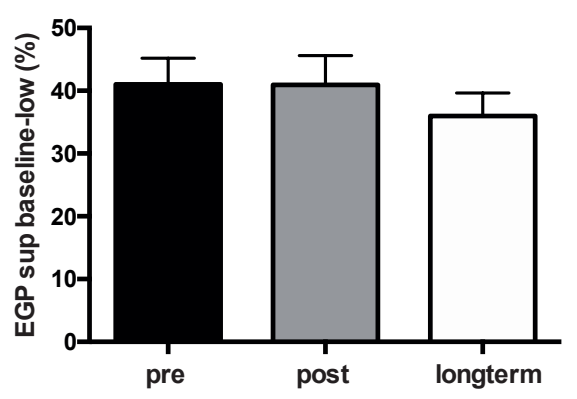

D

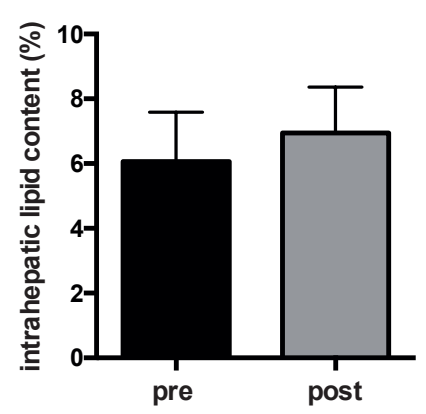

Figure 5. Insulin sensitivity and intrahepatic lipid content

A: Whole body insulin sensitivity. B: Suppression of hepatic endogenous glucose production. C: $\mathrm{HbA} 1 \mathrm{c}$ before the cold acclimation and 2 months after the cold acclimation. D: Intrahepatic lipid content. Black bars represent before cold acclimation, grey bars represent directly after cold acclimation, white bars represent ten days after the cold acclimation, dashed bar represents two months after the cold acclimation. Data are shown as mean \pm SE. 


\section{Insulin sensitivity}

We previously demonstrated that cold acclimation improved insulin sensitivity when measured directly after ten days of cold acclimation (13). Here, we aimed to investigate if this increase in insulin sensitivity would be sustained for ten days after cessation of the cold acclimation intervention. Interestingly, plasma insulin levels during the high insulin phase of the clamp were significantly different between test days $(p=0.02)$ with significantly higher values directly after the cold acclimation compared to before cold acclimation ( $p=0.02$, Supplementary Table 3 ), and therefore we calculated $\mathrm{Si}$ as a measure of insulin sensitivity. However, whole body insulin stimulated glucose uptake ( $\mathrm{Rd}$ glucose high insulin minus baseline) corrected for plasma insulin levels during the clamp (Si), was not different before and directly after cold acclimation nor on the longterm ( $p=0.53$, Figure $5 A)$. Hepatic insulin sensitivity during the low insulin phase was also not affected by cold acclimation ( $p=0.28$, Figure $5 \mathrm{~B}$ ), however during the high insulin phase, EGP suppression was significantly lower directly after cold acclimation compared to before and on the longterm (pre $88.06 \pm$ $7.00 \%$ vs. post $77.60 \pm 7.45 \%$ vs. Iongterm $88.39 \pm 3.44 \%, p=0.04$ ). HbA1c, a marker of long-term glucose homeostasis, was not different before cold acclimation compared to two months after cold acclimation (pre $7.3 \pm 0.3 \%$ vs. two months later $7.2 \pm 0.3 \%, p=0.71$, Figure $5 \mathrm{C})$.

\section{Skeletal muscle GLUT4 translocation}

The lack of effect of cold induced non-shivering thermogenesis on insulin sensitivity contrasts our previous study, in which we found that the increased insulin sensitivity was due to enhanced GLUT4 translocation in skeletal muscle (13). Consistent with a lack of effect of cold induced NST on insulin sensitivity, GLUT4 intensity at the membrane measured in muscle biopsies taken in the non-insulin stimulated condition, was also not affected by cold induced NST (pre $27.37 \pm 3.05$ vs. post $27.63 \pm 2.28$ vs. longterm $27.61 \pm 3.30$ A.U., $p=0.77, n=7$, Figure 6B). Cytosolic GLUT4 intensity was not changed either (pre $20.21 \pm 1.89$ vs. post $20.71 \pm 1.33$ vs. long term $20.46 \pm$ 1.90 A.U., $p=0.96)$.

\section{Skeletal muscle shivering markers}

Because we could not replicate our previously reported positive effects of cold acclimation on insulin sensitivity and GLUT4 translocation (13), we decided to investigate if (the lack of) shivering may have contributed to the discrepancy in glucose homeostasis results between both studies. As a first indication, we see a distinct lack of self-reported shivering in the current study as compared to the previous one (13), which is visualized in Figure 6A. Next, we measured mRNA expression of eight genes that we recently identified (unpublished data) to be 
associated with muscle activation and -remodeling by comparing gene expression patterns - using micro array analysis - between our previous cold acclimation study (13) and an exercise training study (14). As can be seen in Figure 6C, the change in mRNA expression of alpha actin cardiac muscle (ACTC1), cholinergic receptor nicotinic delta subunit (CHRND), coiled-coil domain containing 80 (CCDC80) and cholinergic receptor nicotinic alpha 1 subunit (CHRNA1), upon ten days of cold acclimation different between the two cold-acclimation studies, with basically no change in these genes in the current study (ACTC1 $-0.2 \pm 0.1$ vs. $1.3 \pm 1.8, p=0.02$; CHRND $-0.0 \pm 0.1$ vs. $3.4 \pm 2.5, p=0.02$; CCDC80 $0.1 \pm 0.1$ vs. $3.8 \pm 3.1 p=0.02$; CHRNA1 $-0.1 \pm 0.1$ vs. $2.2 \pm 1.9$ arb. unit, $p=0.02$, in the current and the Hanssen et al. study (13) respectively, Figure 6C). Although similar differences were observed for RRAD, MYH8, LYRM3 and CCDC39, these differences did not reach statistical significance $(p=0.12, p=0.17, p=0.35$ and $p=0.09$ respectively, Figure $6 C$ ).

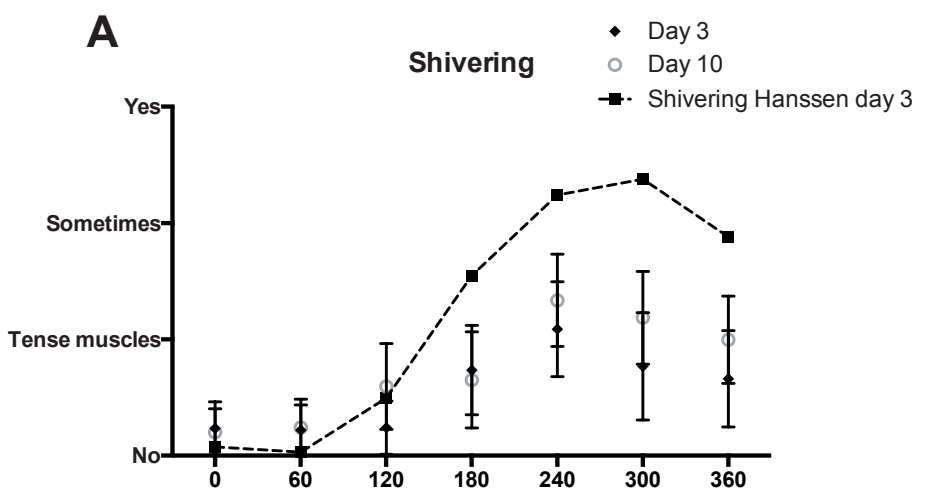

B

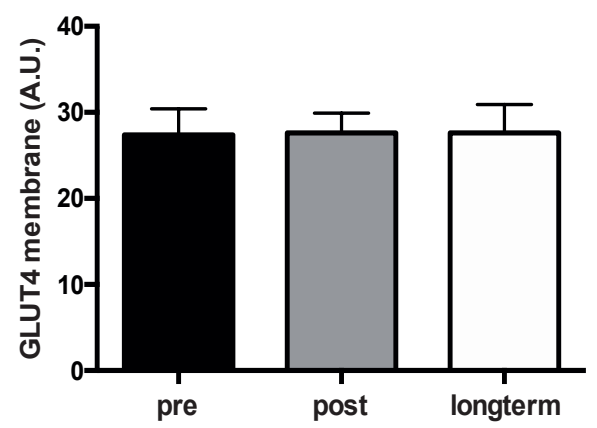

C

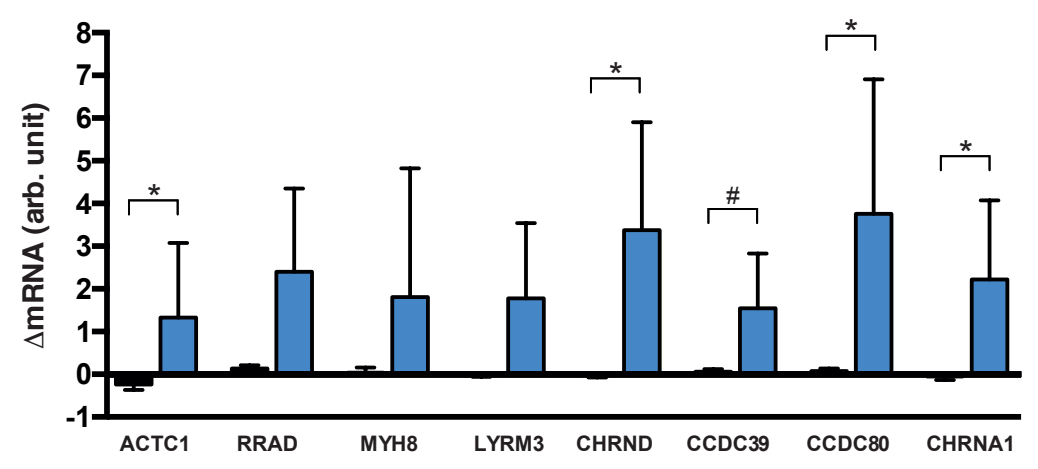

Figure 6. Skeletal muscle shivering markers

A: Self-reported shivering measured with VAS scales at selected timepoints (from $T=0$ minutes until $\mathrm{T}=360$ minutes) during day 3 and day 10 of the cold acclimation period, shown as AUC. The dashed line indicates the self-reported shivering AUC from Hanssen et al. (13) at day 3 B: GLUT4 intensity at the skeletal muscle membrane, expressed as arbitrary units (A.U.). C: Delta gene expression shivering markers, measured in muscle samples from the current study in black $(n=9)$ and from Hanssen et al. (13) in blue $(n=7)$ before and directly after cold acclimation. Data are shown as mean \pm SE. ${ }^{*} \mathrm{p}<0.05, \# p<0.10$. 


\section{DISCUSSION}

Previous studies investigating cold acclimation in humans have shown a potential to treat obesity and T2DM via an increase in energy expenditure (4-7, 12, 13, 36-38) and insulin sensitivity (13), acting through NST in brown adipose tissue and skeletal muscle. Therefore, we hypothesized that cold acclimation with NST could also be beneficial for postprandial metabolism and reduce cardiovascular risk. To this end, we primarily investigated the effects of ten-day cold acclimation without shivering on cardiovascular risk markers, including postprandial glucose and lipid metabolism and markers for arterial stiffness. Moreover, we investigated the long-term effects of cold acclimation without shivering on insulin sensitivity.

Acute cold exposure with and without shivering has frequently been shown to increase energy expenditure, reflected by an increase in basal metabolic rate $(4,5$, 10, 11, 37-42). Yet, consistent with other previous reports $(4,6,7,12,13,36,38,43)$, we did not observe an increase in basal metabolic rate, measured under thermoneutral conditions after cold acclimation with NST. However, our results show a change in fasting substrate selection after cold acclimation with NST, with a decrease in fat oxidation and an increase in carbohydrate oxidation. Furthermore, as also reported before (12), postprandial energy expenditure, which reflects diet induced thermogenesis, was higher in the meal test after cold acclimation compared than in the meal test prior to cold acclimation. Other evidence suggests that acute cold exposure $(5,11,16,39,41)$, but not cold acclimation (5), increases glucose oxidation, fat oxidation and lipid clearance. In agreement with previous work (5, 42), we did not observe changes in fasting plasma lipid levels, postprandial substrate oxidation or postprandial triglyceride response. We only observed a small, albeit significant decrease in plasma fatty acid levels during the first step of the meal test after cold acclimation. Furthermore, no effects of cold acclimation with NST were observed on fasted plasma glucose and insulin levels, consistent with findings after acute cold exposure (42). These results indicate that cold acclimation under nonshivering conditions only had marginal effects on postprandial glucose and lipid metabolism.

Furthermore, we observed a significant improvement in the augmentation index, indicating an indirect reduction in arterial stiffness. However, we did not see any change in $P W V_{\text {c-f. }}$. This indicates that the effects of ten days of cold acclimation with NST might only affect peripheral resistance arteries and not stiffness of the large elastic arteries, such as the aorta. It is likely that the study period of ten days was too short to induce pronounced effects on arterial vessel wall properties. Alternatively, 
cold exposure can increase blood pressure - a main determinant of the $\mathrm{PWV}_{\mathrm{c}-\mathrm{f}}-$ through vasoconstriction (44), and an increased blood pressure during cold exposure could mitigate potential beneficial effects that could be expected from the cold acclimation.

We previously showed marked effects of cold acclimation on insulin sensitivity in T2DM patients (13). Here we aimed to investigate if these effects would be retained for a longer period of time after the last cold exposure under non-shivering conditions. Thus, we performed a hyperinsulinemic euglycemic clamp before cold acclimation, directly (one day) after cold acclimation and ten days after the last cold exposure. Surprisingly, we could not replicate the increase in insulin sensitivity directly following the ten-day cold acclimation period, and also no effects on the long term were observed. Since the increase in insulin sensitivity in our previous study (13) was very marked (40\% increase), observed in all participants, and was accompanied by marked increases in muscle GLUT4 translocation, we carefully evaluated the differences between the two studies. Thus, in both studies patients with T2DM were investigated before and after ten days of cold exposure, following a similar design. One difference in the current study design was the inclusion of a meal test as the primary outcome parameter. This meal test was performed on the day following the ten-day cold exposure. Consequently, the clamp in the current study was performed three days after the meal test, with two days of additional cold exposure in between. We can hence not exclude a carry-over effect of the meal test on the results of the clamp. It should be noted though, that postprandial glucose metabolism measured during the meal tests, which can be seen as a marker of insulin sensitivity, was also not affected by cold acclimation.

Another difference between the two studies was that in the current study we specifically aimed to investigate the effect of NST and aimed to prevent shivering. To this end, the room temperature during cold exposure was approximately $1.4^{\circ} \mathrm{C}$ higher compared to our previous study (13). Furthermore, participants were provided with extra clothing when shivering occurred to prevent shivering thermogenesis. While this strategy was more effective in preventing shivering and/or tense muscle compared to our previous study (13), as indicated by the self-reported shivering questionnaires taken during the cold acclimation, it may also have affected the clamp outcome. Furthermore, we previously reported a marked increase in GLUT4 translocation in the overnight fasted state (so in the absence of elevated insulin levels) and under thermoneutral conditions after cold acclimation $(7,13)$. Classically, this increase in GLUT4 in the cell membrane in the absence of insulin stimulation is attributed to muscle contraction, which is required for shivering or to increase muscle tension (45). 
Consistent with the self-reported absence of shivering, no effect of cold acclimation on GLUT4 translocation was found in the current study. To further investigate the possible effects of shivering that we may have missed in our previous study (13), we performed gene-expression analyses in skeletal muscle biopsies obtained before and directly after the cold acclimation in both studies. Results of these analyses show upregulation of muscle-contraction-related-genes in our previous study (13) but no change in the current study. Those genes were selected from micro-array data obtained after cold exposure (13) and compared with an exercise training study (14). This comparison revealed overlap in skeletal muscle gene expression related to muscle contraction pathways (unpublished data). Interestingly, all previous published studies that observed improvements in insulin sensitivity after cold acclimation, included at least a few days of (mild) shivering before NST occurred $(4,5,7,13,38)$. Taken together, the findings reported here and in literature $(4,10,46)$, suggest that some mild form of cold-induced shivering is needed to trigger beneficial effects on skeletal muscle insulin sensitivity.

In conclusion, we here show that a ten-day cold acclimation period at $16-17^{\circ} \mathrm{C}$ with NST does not induce metabolic improvements nor reduce cardiovascular risk markers in obese men and women with T2DM. The lack of effects in the current study are probably due to the absence of shivering compared to previous studies. Therefore, further research could focus on the potential of (mild) shivering thermogenesis on metabolic health, as ten days of cold acclimation without shivering at $16-17{ }^{\circ} \mathrm{C}$ in obese men and women with T2DM is insufficient to induce positive effects on human metabolism. 


\section{SUPPLEMENTARY MATERIAL FOR CHAPTER 4}

Supplementary Table 1. Participant characteristics regarding diabetes

\begin{tabular}{|c|c|c|}
\hline Participant & Diabetes duration (years) & Diabetes medication \\
\hline 1 & 15 & $\begin{array}{l}\text { Metformin } 850 \mathrm{mg}, 3 x / \text { day } \\
\text { Gliclazide } 80 \mathrm{mg}, 2 x / \text { day }\end{array}$ \\
\hline 2 & 14 & Metformin 500mg, 2x/day \\
\hline 3 & 15 & $\begin{array}{l}\text { Metformin } 1000 \mathrm{mg}, 2 x / \text { day } \\
\text { Gliclazide } 30 \mathrm{mg}, 2 x / \text { day }\end{array}$ \\
\hline 4 & 6 & Metformin 1000mg, 3x/day \\
\hline 5 & 8 & $\begin{array}{l}\text { Metformin } 850 \mathrm{mg} 1 \times / \text { day } \\
\text { Glimepiride } 2 \mathrm{mg}, 1 \times / \text { day } \\
\text { Liraglutide } 6 \mathrm{mg}, 18 \mathrm{eh}, 1 \times \text { /day }\end{array}$ \\
\hline 6 & 5 & Metformin 850mg, 2x/day \\
\hline 7 & 9 & Metformin 500mg, 2x/day \\
\hline 8 & 7 & $\begin{array}{l}\text { Metformin 500mg, 3x/day } \\
\text { Gliclazide 30mg, 1x/day }\end{array}$ \\
\hline 9 & 1 & Gliclazide 80mg, 1x/day \\
\hline
\end{tabular}

Supplementary Table 2. Nutritional information of meal test shake

\begin{tabular}{|lll|}
\hline & Content & Energy \% \\
\hline Energy & $755 \mathrm{kcal}$ & 100 \\
Fat & $50.8 \mathrm{gr}$ & 60.6 \\
$\quad$ Saturated fat & $26.1 \mathrm{gr}$ & 31.1 \\
Unsaturated fat & $23.8 \mathrm{gr}$ & 28.4 \\
Cholesterol & $0.9 \mathrm{gr}$ & 1.1 \\
Carbohydrate & $62.3 \mathrm{gr}$ & 33.0 \\
Protein & $10.9 \mathrm{gr}$ & 5.8 \\
\hline
\end{tabular}


Supplementary Table 3. Plasma levels and substrate kinetics measured during the clamps

\begin{tabular}{|c|c|c|c|c|}
\hline Parameter & Clamp pre & Clamp post & Clamp longterm & $\mathrm{p}$-value \\
\hline \multicolumn{5}{|c|}{ Plasma Glucose (mmol/l) } \\
\hline Baseline & $7.77 \pm 0.54$ & $7.98 \pm 0.45$ & $7.91 \pm 0.54$ & 0.99 \\
\hline Low insulin & $5.99 \pm 0.25$ & $5.78 \pm 0.31$ & $5.63 \pm 0.17$ & 0.21 \\
\hline High insulin & $5.21 \pm 0.12$ & $5.11 \pm 0.11$ & $5.04 \pm 0.08$ & 0.69 \\
\hline \multicolumn{5}{|c|}{ Plasma Insulin (mU/l) } \\
\hline Baseline & $6.32 \pm 1.992$ & $6.99 \pm 1.48$ & $6.56 \pm 1.71$ & 0.69 \\
\hline Low insulin & $14.09 \pm 1.87$ & $12.64 \pm 1.56$ & $12.62 \pm 0.88$ & 0.57 \\
\hline High insulin & $62.50 \pm 3.24 c$ & $59.34 \pm 4.13$ & $56.03 \pm 2.68 \mathrm{C}$ & $0.02^{*}$ \\
\hline \multicolumn{5}{|c|}{ Energy expenditure (kJ/min) } \\
\hline Baseline & $4.82 \pm 0.25$ & $4.91 \pm 0.27$ & $4.86 \pm 0.20$ & 0.79 \\
\hline Low insulin & $4.86 \pm 0.34$ & $4.92 \pm 0.28$ & $4.75 \pm 0.24$ & 0.24 \\
\hline High insulin & $4.67 \pm 0.30^{\mathrm{B}}$ & $4.89 \pm 0.27^{\mathrm{B}, \mathrm{D}}$ & $4.67 \pm 0.18^{D}$ & $0.02^{*}$ \\
\hline \multicolumn{5}{|c|}{ Carbohydrate oxidation ( $\mu \mathrm{mol} / \mathrm{kg} / \mathrm{min})$} \\
\hline Baseline & $3.76 \pm 0.47^{A}$ & $4.97 \pm 0.68^{A}$ & $4.68 \pm 0.68$ & $<0.01^{\star \star}$ \\
\hline Low insulin & $7.27 \pm 0.79$ & $7.83 \pm 0.84$ & $7.15 \pm 0.77$ & 0.79 \\
\hline High insulin & $10.36 \pm 1.15$ & $11.07 \pm 1.21$ & $9.73 \pm 1.41$ & 0.30 \\
\hline \multicolumn{5}{|c|}{ Fatty acid oxidation ( $\mu \mathrm{mol} / \mathrm{kg} / \mathrm{min})$} \\
\hline Baseline & $3.80 \pm 0.17^{A}$ & $3.57 \pm 0.17^{A, D}$ & $3.71 \pm 0.19^{D}$ & $<0.01^{\star \star}$ \\
\hline Low insulin & $2.81 \pm 0.23$ & $2.86 \pm 0.24$ & $2.96 \pm 0.16$ & 0.87 \\
\hline High insulin & $2.14 \pm 0.24$ & $2.16 \pm 0.21$ & $2.39 \pm 0.26$ & 0.99 \\
\hline
\end{tabular}

Abbreviations: Ra, rate of appearance; Rd, rate of disappearance; $S_{i}$, whole body insulin sensitivity; EGP, endogenous glucose production; NOGD, non-oxidative glucose disposal; FFA, free fatty acids. Data are expressed as mean $\pm S E .{ }^{* *} p<0.01,{ }^{*} p<0.05$, ${ }^{A}$ significant difference $(p<0.01)$ between Clamp pre and Clamp post, ${ }^{B}$ significant difference $(p<0.05)$ between Clamp pre and Clamp post, ${ }^{C}$ significant difference $(p<0.05)$ between Clamp pre and Clamp post, ${ }^{D}$ trend in difference $(p<0.10)$ between Clamp post and Clamp longterm. 


\section{Chapter 4}

Supplementary Table 4. Plasma levels and substrate kinetics measured during the meal test

\begin{tabular}{|c|c|c|c|}
\hline Parameter & Meal test pre & Meal test post & p-value \\
\hline \multicolumn{4}{|l|}{ Plasma Glucose } \\
\hline AUC & $4433 \pm 236$ & $4285 \pm 170$ & 0.43 \\
\hline iAUC & $860 \pm 171$ & $747 \pm 159$ & 0.25 \\
\hline \multicolumn{4}{|l|}{ Plasma Insulin } \\
\hline AUC & $15826 \pm 1550$ & $15640 \pm 1458$ & 0.65 \\
\hline iAUC & $9457 \pm 1097$ & $9060 \pm 1119$ & 0.36 \\
\hline \multicolumn{4}{|l|}{ Plasma Triglycerides } \\
\hline AUC & $1339 \pm 109$ & $1411 \pm 123$ & 0.50 \\
\hline iAUC & $655 \pm 89$ & $664 \pm 67$ & 0.36 \\
\hline \multicolumn{4}{|l|}{ Plasma Free fatty acids } \\
\hline AUC & $214852 \pm 9104$ & $202701 \pm 10011$ & 0.16 \\
\hline iAUC & $13182 \pm 6077$ & $23031 \pm 7650$ & 0.20 \\
\hline AUC first meal & $94228 \pm 5115$ & $86014 \pm 4545$ & $0.04^{\star}$ \\
\hline AUC second meal & $120624 \pm 5448$ & $106486 \pm 11976$ & 0.30 \\
\hline \multicolumn{4}{|c|}{ AUC Energy expenditure } \\
\hline AUC & $2620 \pm 145$ & $2752 \pm 168$ & $0.03^{*}$ \\
\hline iAUC & $316 \pm 37$ & $355 \pm 22$ & 0.69 \\
\hline \multicolumn{4}{|c|}{ AUC Carbohydrate oxidation } \\
\hline AUC & $3328 \pm 371$ & $3866 \pm 550$ & 0.44 \\
\hline iAUC & $726 \pm 152$ & $998 \pm 103$ & 0.22 \\
\hline \multicolumn{4}{|c|}{ AUC Fatty acid oxidation } \\
\hline$A \cup C$ & $1688 \pm 92$ & $1667 \pm 78$ & $>0.99$ \\
\hline iAUC & $611 \pm 159$ & $526 \pm 63$ & 0.94 \\
\hline
\end{tabular}

Abbreviations: AUC, area under the curve; iAUC, incremental area under the curve.

Data are expressed as mean \pm SE. ${ }^{*} p<0.05$. 
A

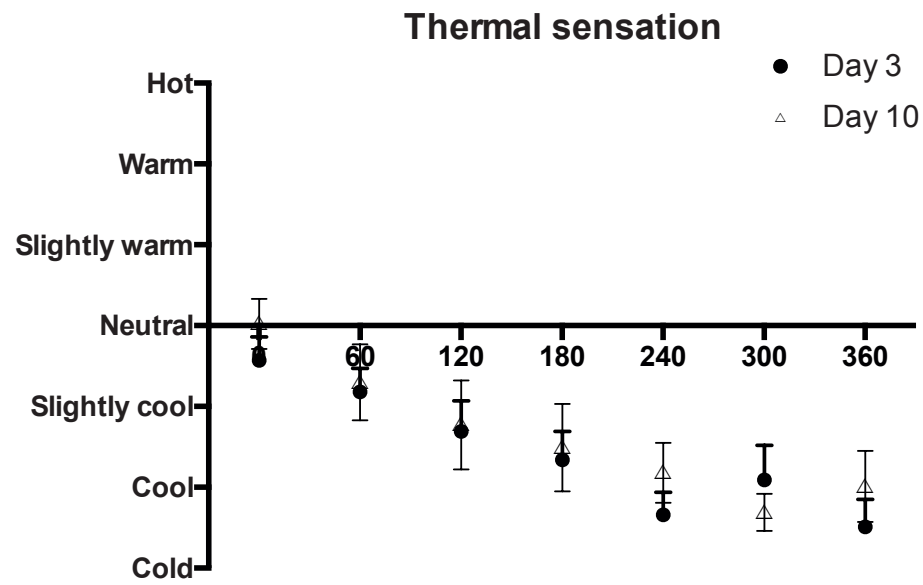

B

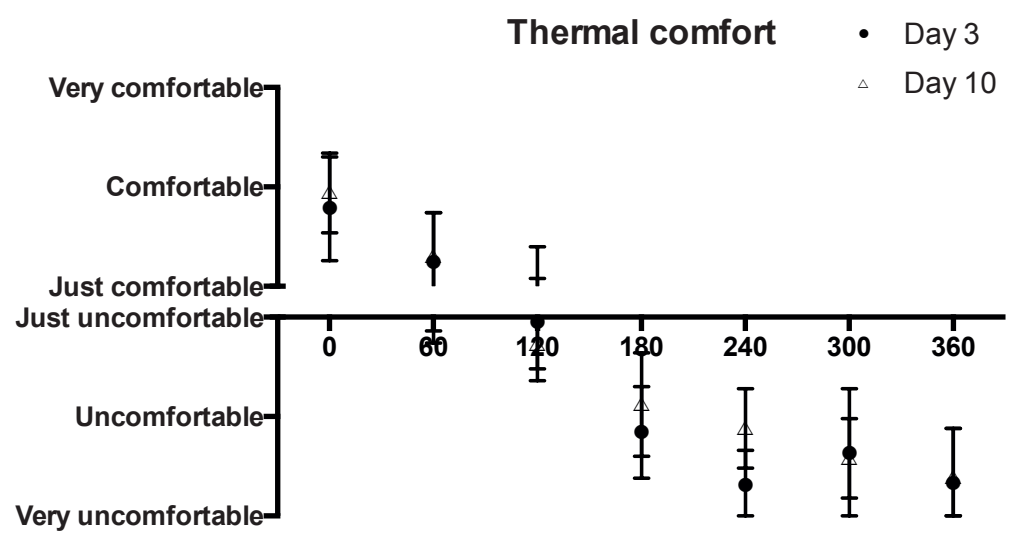

Supplementary Figure 1. Subjective responses to cold acclimation

Self-reported thermal sensation (A) and thermal comfort (B) measured with VAS scales at selected timepoints (from $t=0$ min until $t=360$ min) during day 3 and day 10 of the cold acclimation period, shown as AUC. Data are expressed as mean \pm SE. 


\section{REFERENCES}

1. Kannel WB, McGee DL. Diabetes and cardiovascular disease. The Framingham study. JAMA. 1979;241(19):2035-8.

2. Emerging Risk Factors C, Sarwar N, Gao P, Seshasai SR, Gobin R, Kaptoge S, et al. Diabetes mellitus, fasting blood glucose concentration, and risk of vascular disease: a collaborative meta-analysis of 102 prospective studies. Lancet. 2010;375(9733):2215-22.

3. Davis TRA. Chamber cold acclimatization in man. Journal of Applied Physiology. 1961.

4. Blondin DP, Daoud A, Taylor T, Tingelstad HC, Bezaire V, Richard D, et al. Four-week cold acclimation in adult humans shifts uncoupling thermogenesis from skeletal muscles to brown adipose tissue. J Physiol-London. 2017;595(6):2099-113.

5. Gordon K, Blondin DP, Friesen BJ, Tingelstad HC, Kenny GP, Haman F. Seven days of cold acclimation substantially reduces shivering intensity and increases nonshivering thermogenesis in adult humans. J Appl Physiol. 2019;126(6):1598-606.

6. Blondin DP, Labbe SM, Tingelstad HC, Noll C, Kunach M, Phoenix S, et al. Increased Brown Adipose Tissue Oxidative Capacity in Cold-Acclimated Humans. J Clin Endocr Metab. 2014;99(3):E438-E46.

7. Hanssen MJ, van der Lans AA, Brans B, Hoeks J, Jardon KM, Schaart G, et al. Short-term Cold Acclimation Recruits Brown Adipose Tissue in Obese Humans. Diabetes. 2016;65(5):1179-89.

8. van der Lans AA, Hoeks J, Brans B, Vijgen GH, Visser MG, Vosselman MJ, et al. Cold acclimation recruits human brown fat and increases nonshivering thermogenesis. J Clin Invest. 2013;123(8):3395-403.

9. van Marken Lichtenbelt WD, Vanhommerig JW, Smulders NM, Drossaerts JM, Kemerink GJ, Bouvy ND, et al. Cold-activated brown adipose tissue in healthy men. $\mathrm{N}$ Engl $\mathrm{J}$ Med. 2009;360(15):1500-8.

10. Wijers SLJ, Schrauwen P, Saris WHM, Lichtenbelt WDV. Human Skeletal Muscle Mitochondrial Uncoupling Is Associated with Cold Induced Adaptive Thermogenesis. Plos One. 2008;3(3).

11. Chondronikola M, Volpi E, Borsheim E, Porter C, Annamalai P, Enerback S, et al. Brown adipose tissue improves whole-body glucose homeostasis and insulin sensitivity in humans. Diabetes. 2014;63(12):4089-99.

12. Lee P, Smith S, Linderman J, Courville AB, Brychta RJ, Dieckmann W, et al. TemperatureAcclimated Brown Adipose Tissue Modulates Insulin Sensitivity in Humans. Diabetes. 2014;63(11):3686-98.

13. Hanssen MJ, Hoeks J, Brans B, van der Lans AA, Schaart G, van den Driessche JJ, et al. Shortterm cold acclimation improves insulin sensitivity in patients with type 2 diabetes mellitus. Nat Med. 2015;21(8):863-5.

14. Meex RC, Schrauwen-Hinderling VB, Moonen-Kornips E, Schaart G, Mensink M, Phielix E, et al. Restoration of muscle mitochondrial function and metabolic flexibility in type 2 diabetes by exercise training is paralleled by increased myocellular fat storage and improved insulin sensitivity. Diabetes. 2010;59(3):572-9.

15. Arca M, Pigna G, Favoccia C. Mechanisms of diabetic dyslipidemia: relevance for atherogenesis. Curr Vasc Pharmacol. 2012;10(6):684-6.

16. Bartelt A, Bruns OT, Reimer R, Hohenberg H, Ittrich H, Peldschus K, et al. Brown adipose tissue activity controls triglyceride clearance. Nat Med. 2011;17(2):200-5.

17. Chondronikola M, Volpi E, Borsheim E, Porter C, Saraf MK, Annamalai P, et al. Brown Adipose Tissue Activation Is Linked to Distinct Systemic Effects on Lipid Metabolism in Humans. Cell Metab. 2016;23(6):1200-6.

18. Ceriello A, Taboga C, Tonutti L, Quagliaro L, Piconi L, Bais B, et al. Evidence for an independent and cumulative effect of postprandial hypertriglyceridemia and hyperglycemia on endothelial dysfunction and oxidative stress generation: effects of short- and long-term simvastatin treatment. Circulation. 2002;106(10):1211-8.

19. Vosselman MJ, Hoeks J, Brans B, Pallubinsky H, Nascimento EB, van der Lans AA, et al. Low brown adipose tissue activity in endurance-trained compared with lean sedentary men. Int $J$ Obes (Lond). 2015;39(12):1696-702.

20. Baumgartner S, Mensink RP, Plat J. Effects of a Plant Sterol or Stanol Enriched Mixed Meal on Postprandial Lipid Metabolism in Healthy Subjects. PLoS One. 2016;11(9):e0160396.

21. Joris PJ, Plat J, Bakker SJ, Mensink RP. Long-term magnesium supplementation improves arterial stiffness in overweight and obese adults: results of a randomized, double-blind, placebo-controlled intervention trial. Am J Clin Nutr. 2016;103(5):1260-6. 
22. Joris PJ, Plat J, Kusters YH, Houben AJ, Stehouwer CD, Schalkwijk CG, et al. Diet-induced weight loss improves not only cardiometabolic risk markers but also markers of vascular function: a randomized controlled trial in abdominally obese men. Am J Clin Nutr. 2017;105(1):23-31.

23. Hubbard LD, Brothers RJ, King WN, Clegg LX, Klein R, Cooper LS, et al. Methods for evaluation of retinal microvascular abnormalities associated with hypertension/sclerosis in the Atherosclerosis Risk in Communities Study. Ophthalmology. 1999;106(12):2269-80.

24. DeFronzo RA, Tobin JD, Andres R. Glucose clamp technique: a method for quantifying insulin secretion and resistance. The American journal of physiology. 1979;237(3):E214-23.

25. de Ligt M, Bruls YMH, Hansen J, Habets MF, Havekes B, Nascimento EBM, et al. Resveratrol improves ex vivo mitochondrial function but does not affect insulin sensitivity or brown adipose tissue in first degree relatives of patients with type 2 diabetes. Mol Metab. 2018;12:39-47.

26. Steele R. Influences of glucose loading and of injected insulin on hepatic glucose output. Annals of the New York Academy of Sciences. 1959;82:420-30.

27. Bergman RN, Finegood DT, Ader M. Assessment of insulin sensitivity in vivo. Endocr Rev. 1985;6(1):45-86.

28. Peronnet F, Massicotte D. Table of nonprotein respiratory quotient: an update. Canadian journal of sport sciences = Journal canadien des sciences du sport. 1991;16(1):23-9.

29. Weir JB. New methods for calculating metabolic rate with special reference to protein metabolism. The Journal of physiology. 1949;109(1-2):1-9.

30. Lindeboom L, Nabuurs CI, Hesselink MK, Wildberger JE, Schrauwen P, Schrauwen-Hinderling VB. Proton magnetic resonance spectroscopy reveals increased hepatic lipid content after a single high-fat meal with no additional modulation by added protein. Am J Clin Nutr. 2015;101(1):65-71.

31. Bergstrom J, Hermansen L, Hultman E, Saltin B. Diet, muscle glycogen and physical performance. Acta Physiol Scand. 1967;71(2):140-50.

32. Schneider CA, Rasband WS, Eliceiri KW. NIH Image to ImageJ: 25 years of image analysis. Nature Methods. 2012;9:671-5.

33. van Moorsel D, Hansen J, Havekes B, Scheer F, Jorgensen JA, Hoeks J, et al. Demonstration of a day-night rhythm in human skeletal muscle oxidative capacity. Mol Metab. 2016;5(8):63545.

34. Wefers J, van Moorsel D, Hansen J, Connell NJ, Havekes B, Hoeks J, et al. Circadian misalignment induces fatty acid metabolism gene profiles and compromises insulin sensitivity in human skeletal muscle. Proc Natl Acad Sci U S A. 2018;115(30):7789-94.

35. Friedewald WT, Levy RI, Fredrickson DS. Estimation of the concentration of low-density lipoprotein cholesterol in plasma, without use of the preparative ultracentrifuge. Clinical chemistry. 1972;18(6):499-502.

36. van der Lans AAJJ, Hoeks J, Brans B, Vijgen GHEJ, Visser MGW, Vosselman MJ, et al. Cold acclimation recruits human brown fat and increases nonshivering thermogenesis. J Clin Invest. 2013;123(8):3395-403.

37. Yoneshiro T, Aita S, Matsushita M, Kayahara T, Kameya T, Kawai Y, et al. Recruited brown adipose tissue as an antiobesity agent in humans. J Clin Invest. 2013;123(8):3404-8.

38. Blondin DP, Tingelstad HC, Noll C, Frisch F, Phoenix S, Guerin B, et al. Dietary fatty acid metabolism of brown adipose tissue in cold-acclimated men. Nat Commun. 2017;8.

39. Vallerand AL, Zamecnik J, Jones PJH, Jacobs I. Cold stress increases lipolysis, FFA R-a and TG/FFA cycling in humans. Aviat Space Envir Md. 1999;70(1):42-50.

40. van Ooijen AMJ, Lichtenbelt WDVM, van Steenhoven AA, Westerterp KR. Seasonal changes in metabolic and temperature responses to cold air in humans. Physiol Behav. 2004;82(2-3):54553.

41. Chondronikola M, Volpi E, Borsheim E, Porter C, Saraf MK, Annamalai P, et al. Brown Adipose Tissue Activation Is Linked to Distinct Systemic Effects on Lipid Metabolism in Humans. Cell Metab. 2016;23(6):1200-6.

42. Iwen KA, Backhaus J, Cassens M, Waltl M, Hedesan OC, Merkel M, et al. Cold-Induced Brown Adipose Tissue Activity Alters Plasma Fatty Acids and Improves Glucose Metabolism in Men. J Clin Endocr Metab. 2017;102(11):4226-34.

43. Plasqui G, Kester AD, Westerterp KR. Seasonal variation in sleeping metabolic rate, thyroid activity, and leptin. Am J Physiol Endocrinol Metab. 2003;285(2):E338-43.

44. Kingma BR, Frijns AJ, Saris WH, van Steenhoven AA, Lichtenbelt WD. Increased systolic blood pressure after mild cold and rewarming: relation to cold-induced thermogenesis and age. Acta Physiol (Oxf). 2011;203(4):419-27. 
45. Douen AG, Ramlal T, Rastogi S, Bilan PJ, Cartee GD, Vranic M, et al. Exercise induces recruitment of the "insulin-responsive glucose transporter". Evidence for distinct intracellular insulin- and exercise-recruitable transporter pools in skeletal muscle. J Biol Chem. 1990;265(23): 13427-30.

46. Bal NC, Maurya SK, Singh S, Wehrens XHT, Periasamy M. Increased Reliance on Musclebased Thermogenesis upon Acute Minimization of Brown Adipose Tissue Function. Journal of Biological Chemistry. 2016;291(33):17247-57. 



\section{CHAPTER 5}

Replacing sedentary time with standing and walking is similar effective in enhancing insulin sensitivity as moderate-tovigorous exercise

Carlijn M.E. Remie, Lena Bilet, Bernard M.F.M. Duvivier, Maaike Bergman, Kay H.M. Roumans, Stephanie M.D. Huysmans, Evelyn Zacharewicz, Vera H.W. de Wit, Niels J. Connell, Bas Havekes, Johanna A. Jörgensen, Kim Brouwers, Hans H.C.M. Savelberg Vera B. Schrauwen-Hinderling, Joris Hoeks, Matthijs K.C. Hesselink, Esther Phielix, Patrick Schrauwen

In preparation

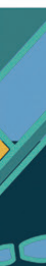




\section{ABSTRACT}

Background \& Aims: Sedentary behavior has been associated with an increased risk to develop type 2 diabetes, metabolic syndrome and cardiovascular diseases. Replacing sitting time by activities such as standing and walking or by physical exercise have both been proven effective in improving aspects of metabolic health, including glucose homeostasis. If these two physical activity regimes act via similar mechanisms remains however to be investigated.

Methods: A randomized, cross-over intervention study was conducted in twelve overweight/obese women. Participants performed three activity regimes of each four days: 1) sit regime (sitting $14 \mathrm{~h} /$ day), 2) exercise regime (sitting $13 \mathrm{~h} /$ day, exercise 1 h/day) and 3) sit less regime (sitting 9 h/day, standing 4 h/day and walking 3 h/day). After each regime insulin sensitivity was determined using a hyperinsulinemiceuglycemic clamp combined with muscle biopsies, intrahepatic lipid content was determined by MRS and substrate oxidation by indirect calorimetry. Ex vivo mitochondrial function in muscle biopsies was measured using high-resolution respirometry.

Results: Peripheral insulin sensitivity was significantly higher after the exercise regime compared to the sit regime $(\Delta \mathrm{Rd} 28.2 \pm 3.3 \mu \mathrm{mol} / \mathrm{kg} / \mathrm{min}$ vs. $20.7 \pm 3.7 \mu \mathrm{mol} / \mathrm{kg} / \mathrm{min}$, $\mathrm{p}<0.01)$ and tended to be higher in the sit less regime $(\Delta \mathrm{Rd} 25.5 \pm 2.8 \mu \mathrm{mol} / \mathrm{kg} / \mathrm{min}$, $p=0.08)$. This increase in peripheral insulin sensitivity was mainly due to a higher NOGD after the exercise and sit less regimes compared to the sit regime, with no change in oxidative glucose disposal. Muscle mitochondrial respiratory capacity was unaffected by the three activity regimes. Hepatic insulin sensitivity neither intrahepatic lipid content was different between the three activity regimes.

Conclusion: Replacing sitting time by standing and light walking has comparable positive effects on peripheral insulin sensitivity compared to vigorous exercise, which could not be explained by improvements in mitochondrial function. 


\section{INTRODUCTION}

Sedentary behaviour has frequently been reported to be a risk factor for type 2 diabetes, metabolic syndrome, cardiovascular disease and all-cause mortality (1-3). As such, physical activity or exercise has been an important cornerstone in the treatment of type 2 diabetes. Indeed, a wide variety of exercise interventions including endurance training, resistance training and/or high intensity interval training, or any combination - have been shown to be effective in improving insulin sensitivity and glycemic control (4). Although this treatment strategy has been proven to be very effective, the compliance to maintain an active lifestyle with regular moderate-tovigorous physical activity has been shown to be low in the general European and US adult population $(5,6)$, with even lower compliance in people diagnosed with type 2 diabetes or adults at risk to develop this disease (7).

Alternative, more assessible approaches are therefore needed for the treatment and prevention of type 2 diabetes. Strategies to reduce sedentary behaviour involving light physical activities are generally considered less demanding than structured exercise and hence are more likely to have long-term compliance (8). Indeed, numerous human interventions studies in which daily activities were manipulated, have shown promising effects of reducing sedentary time when replaced with standing $(9,10)$, walking (9-13) or short exercise bouts (13) on postprandial plasma glucose and insulin responses. We recently showed improvements in glycemic control already after a 4-day intervention in which two sitting replacement strategies were compared to a sedentary lifestyle (14-16). Replacing sitting time with several hours of standing and walking was effective in improving glucose homeostasis - as determined by an OGTT - in healthy young adults, healthy obese men and women and patients with type 2 diabetes, when compared to sedentary behaviour (14-16). Moreover, in these studies, replacing one hour of sitting time with one hour of exercise resulted in a near significant reduction of $24 \mathrm{~h}$ glucose iAUC, a reduction that was even more pronounced when sitting time was replaced by several hours of standing and walking (16). At this stage, these promising results for light physical activities provide putative novel tools for improvements in metabolic control of type 2 diabetes.

So far, the exact mechanisms underlying the beneficial effects of breaking sedentary time are unknown. Mechanisms underlying the exercise-induced glucose lowering effect have been well established; it has been shown that next to insulin, muscle contraction is also able to stimulate GLUT4 translocation, thereby facilitating glucose uptake in skeletal muscle $(17,18)$. In addition, is has been shown that exercise training is an effective tool to enhance skeletal muscle mitochondrial function, which 
coincides with better peripheral insulin sensitivity (19). Whether the observed improvements in glucose homeostasis by replacing sedentary time with standing and walking are exerted via similar pathways has not been extensively studied. Analysis in human muscle biopsies, obtained after interrupting prolonged sitting, revealed changes in skeletal muscle gene expression comparable to exercise induced changes $(20,21)$, but no functional measurements were performed. Furthermore, habitual physical activity in daily life has been correlated positively with markers of mitochondrial capacity (22), which might suggest a role for mitochondria in the observed improvement in insulin sensitivity upon sitting less, but data directly testing this hypothesis is lacking

Therefore, in the present study, the primary aim was to investigate whether an improved glucose homeostasis upon the replacement of sedentary time by standing/walking - as observed by Duvivier et al. (14-16) - can be explained by enhanced peripheral insulin sensitivity and skeletal muscle mitochondrial function as potential underlying factors. Furthermore, we investigated whether hepatic insulin sensitivity and hepatic lipid content are affected by replacing sedentary time by standing/walking. These outcomes were also compared to replacing sedentary time by a single exercise bout per day.

\section{METHODS}

\section{Participants}

Recruitment and data collection took place between December 2017 and August 2019 in the vicinity of Maastricht. In total 26 female volunteers were screened, of who thirteen were found eligible and included in the study. Of these, twelve participants completed the study as one participant dropped out because of personal reasons. The screening included an assessment of blood biochemistry, electrocardiography, anthropometry measurements and a questionnaire including Baecke physical activity questionnaire (23). Inclusion criteria were healthy postmenopausal women, 45-70 years of age, BMI 25-35 kg/m², sedentary lifestyle (<3 h exercise per week), nonsmoking for at least 6 months, alcohol use of $\leq 2$ servings per day, stable body weight for at least 6 months.

\section{Study design}

A randomized three-arm cross-over intervention study was performed, as previously published elsewhere (Figure 1) (16). In short, participants underwent all three study arms including four-day activity regimes: 1) sit (SIT), 2) exercise (EXE), 3) sit less (SL). 
Prior to the three activity regimes baseline measurements were performed for participant characterization. During the sit regime, participants were instructed to sit $14 \mathrm{~h} /$ day, stand $1 \mathrm{~h} /$ day, walk $1 \mathrm{~h} /$ day and to spend $8 \mathrm{~h} /$ day sleeping or lying. During the exercise regime $1 \mathrm{~h} /$ day of sitting was replaced by $1 \mathrm{~h} /$ day of vigorous supervised exercise. The rest of the days in the exercise regime were spend similarly as during the sit regime. During the sit less regime $5 \mathrm{~h} /$ day of sitting was replaced by $3 \mathrm{~h}$ of standing and $2 \mathrm{~h}$ of walking. Participants were advised to spread the standing and walking hours over the day. The exercise and sit less regime differed largely in time spent sitting but were designed to have equal total daily energy expenditure. The sit and exercise regime differed only $1 \mathrm{~h}$ in sitting time but had substantially different total daily energy expenditure. Any other physical activities besides sitting, standing, walking and the supervised exercise session were limited as much as possible. Directly after the four-day activity regimes, on day five, several measurements were performed. A washout period of at least nine days and maximum 23 days was applied between the activity regimes.
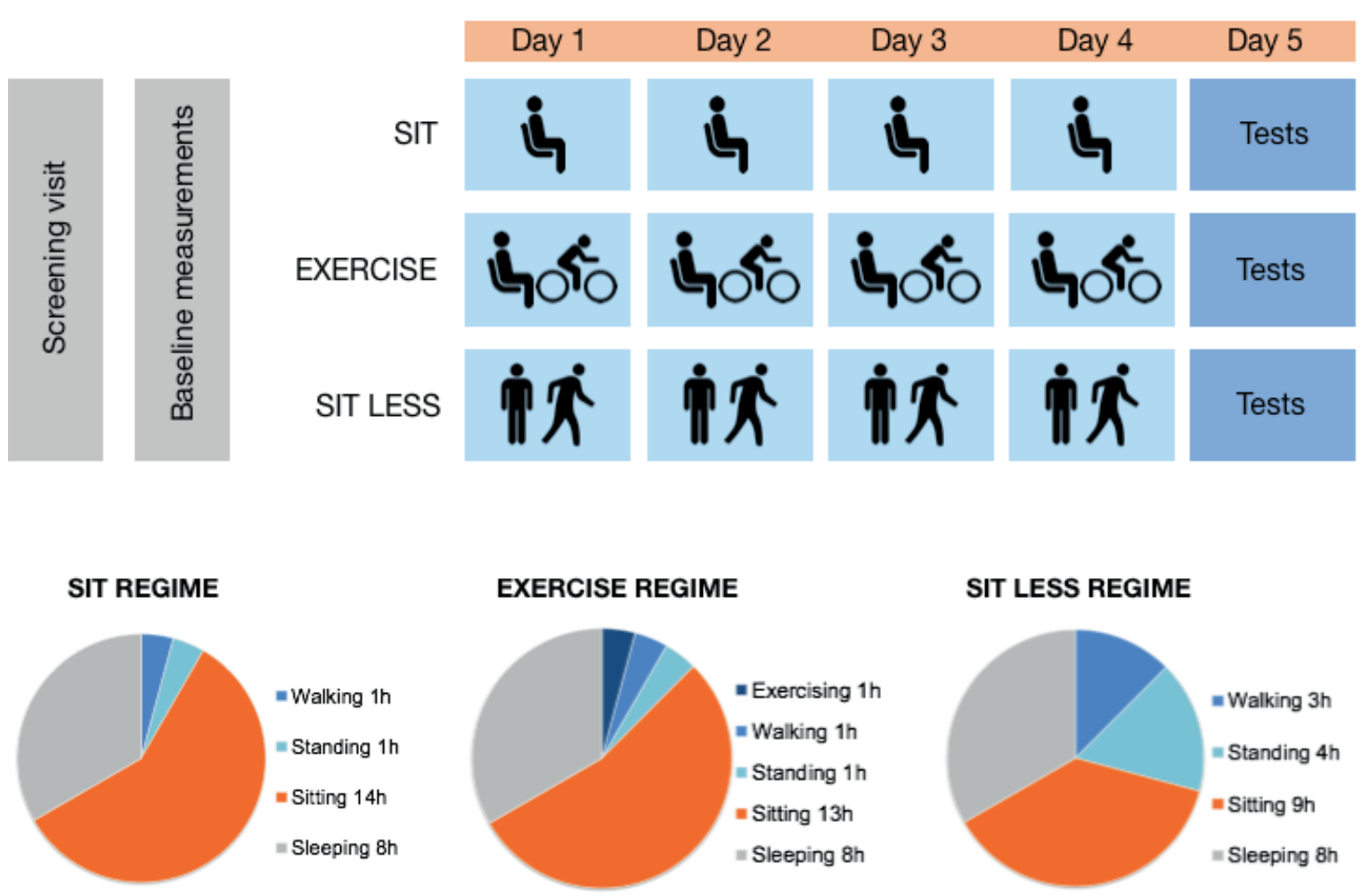

Figure 1. Study design

A randomized three-arm cross-over design, in which participants followed three activity regimes in a random order: sit regime, exercise regime and sit less regime, with a washout period of 9-23 days between arms. Each activity regime lasted four days and measurements were performed on day 5. Time per day (expressed in hours) spend walking, standing, sitting, exercising and sleeping are shown in the figure. 


\section{Standardization of dietary intake}

Dietary intake was kept similar during all three regimes. Participants were instructed to adhere to their normal dietary habits. The consumption of alcohol and caffeine rich drinks were not allowed during the five intervention days. During the first activity regime, participants carefully recorded both time and content of all consumptions in a diary. The researcher returned these dietary records to the participants who were instructed to consume exactly the same diet in the second and third activity regime. In order to standardize the last twelve hours before this test day, participants were instructed to consume a standardized diner on the evening of day four before 20:00 $\mathrm{h}$ and refrain from food and drinks afterwards except for water.

\section{Physical activity assessment}

Physical activity and body posture allocation was measured $24 \mathrm{~h} /$ day using an activPAL activity monitor (PAL technologies, Glasgow, Scotland). The activPAL was waterproofed packed and attached to the anterior thigh of the participant. Before the start of the actual activity regimes, participants first worn the activPAL monitor for five consecutive days, including three week and two weekend days, to assess habitual physical activity level. The activPAL discriminates between time being inactive (sitting or lying) or being active (standing and walking) as well as recorded step number and cadence. In addition, participants recorded their physical activity in a diary and these data were compared with activPAL readouts to formulate tailor-made instructions on how to alter daily activities to those prescribed for the three different activity regimes. This was done in order to guarantee optimal compliance with each activity regime. Sleeping time was determined based on diary data. Daily energy expenditure was estimated using the $24 \mathrm{~h}$ activPAL data expressed in metabolic equivalents (METs) and with separate calculations for the exercise session as described earlier (16).

\section{Participant characteristics}

In order to assess participant characteristics, body mass and body volume were assessed using air-displacement plethysmography using the BodPod device (Cosmed, Italy, Rome) according to the manufacturer's instructions (24), from which body composition (fat mass and fat free mass) was calculated. Waist and hip circumference were measured and the average of 3 values were reported. Next, in vivo maximal oxygen consumption $\left(\mathrm{VO}_{2} \mathrm{max}\right.$ ) and maximal power (Wattmax) were assessed during an incremental exercise test on an ergometer (25). The highest average oxygen consumption over 25 seconds was used as the $\mathrm{VO}_{2} \mathrm{max}$, and the corresponding adjusted wattage as Wattmax. The $\mathrm{VO}_{2} \mathrm{max}$ reflects the participant's physical fitness and $60 \%$ of the Wattmax was applied during the supervised exercise session. All the measurements were performed in the morning after an overnight fast 
of at least 10 hours with a minimum of three days before the start of the activity regimes.

\section{Intrahepatic lipid quantification by MR spectroscopy}

At 07:00 $\mathrm{h}$ in the morning of the test day after ending the activity regime period, proton magnetic resonance spectroscopy ( $\left.{ }^{1} \mathrm{H}-\mathrm{MRS}\right)$ was used to quantify intrahepatic lipid content $(\mathrm{IHL})$. All participants were relocated in a wheelchair from the research facility to and from the radiology department. Measurements were performed on a 3.0T whole body scanner (Achieva Tx, Philips Healthcare, Best, The Netherlands). Spectra were acquired as described before (26). Values are given as T2 corrected ratios of the $\mathrm{CH}_{2}$ peak relative to the unsuppressed water peak, expressed as percentage. One participant failed to undergo this measurement, due to claustrophobia and another participant was excluded due to a technical error.

\section{Skeletal muscle biopsies}

Following the MRS measurement, a muscle biopsy was taken (08:30 h) from the $\mathrm{m}$. vastus lateralis under local anesthesia (1\% lidocaine, without epinephrine) using the Bergström technique (27). Muscle tissue was used for mitochondrial respiratory measurements.

\section{Skeletal muscle mitochondrial respiration}

Muscle tissue obtained with the biopsy was immediately placed in an ice-cold preservation medium (BIOPS, OROBOROS Instruments, Innsbruck, Austria). Muscle fibers were permeabilized using saponin. After permeabilization and several washing steps with an ice-cold mitochondrial respiration buffer (MiR05, OROBOROS Instruments, Innsbruck, Austria), muscle fibers were transferred into the highresolution respirometer (Oxygraph, OROBOROS Instruments, Innsbruck, Austria). Thereafter ex vivo mitochondrial respiration was determined by measuring oxygen consumption rate (pmol/mg wet weight per second) upon addition of several substrates using high-resolution respirometry as previously described (28). All measurements were performed in quadruplicate and traces with a cytochrome $\mathrm{C}$ response above 15\% were excluded, indicating disrupted integrity of the inner mitochondrial membrane. Respirometry could not be determined in one participant due to difficulties with the muscle biopsy.

\section{Plasma triglycerides}

Triglycerides (Sigma, Zwijndrecht, The Netherlands) were analyzed using a Pentra 400 in EDTA plasma from fasting blood samples taken in the morning between 06:30 and 07:00 h. 


\section{Blood pressure}

Resting systolic (SBP) and diastolic (DBP) blood pressure as well as and heart rate $(\mathrm{HR})$ were measured in supine position the morning, every 5 minutes between 09:00 and 09:30 h, at least 10 minutes after taking the muscle biopsy and before the start of the clamp, using a semi-continuous blood pressure monitoring device (OmronX, CEMEX Medische Techniek, Nieuwegein, The Netherlands). The average of the seven measurements were reported.

\section{Hyperinsulinemic euglycemic clamp}

After the ${ }^{1} \mathrm{H}-\mathrm{MRS}$, muscle biopsy and blood pressure measurement, a two-step hyperinsulinemic euglycemic clamp was performed to determine hepatic and peripheral insulin sensitivity (29). During the ${ }^{1} \mathrm{H}-\mathrm{MRS}$ measurement (at 07:00 h) a primed, continuous D-[6.6- ${ }^{2} \mathrm{H}_{2}$ ] glucose tracer infusion was started $(0.04 \mathrm{mg} / \mathrm{kg} / \mathrm{min})$. Upon $3 \mathrm{~h}$ of D2-glucose pre-infusion, a $3 \mathrm{~h}$ primed low dose continuous insulin infusion was started (10 $\mathrm{mU} / \mathrm{m}^{2}$ per $\mathrm{min}$ ) to assess hepatic insulin sensitivity. Subsequently, a $2.5 \mathrm{~h}$ primed high dose continuous insulin infusion was started ( $40 \mathrm{mU} / \mathrm{m}^{2}$ per $\mathrm{min}$ ) to measure peripheral insulin sensitivity. Blood was frequently sampled from arterialized blood to monitor glucose levels in order to maintain euglycemia ( $5.0-5.5 \mathrm{mmol} / \mathrm{l})$. During the last 30 minutes of the baseline, low and high insulin phases, steady state was reached and blood samples were collected for determination of glucose tracer kinetics. During these phases also whole body substrate utilization was measured using indirect calorimetry (Omnical, Maastricht Instruments, Maastricht, The Netherlands) (30). Due to technical reasons, one participant was excluded from the low insulin phase clamp analysis and another participant was excluded from the high insulin phase clamp analysis.

\section{Calculations}

During the clamp, energy expenditure, carbohydrate $(\mathrm{CHO})$ oxidation and fatty acid (FA) oxidation rates were calculated using equations based on the measured oxygen and carbon dioxide concentrations with the assumption that protein oxidation was negligible $(31,32)$. The respiratory exchange ratio (RER) was calculated by the carbon dioxide/oxygen ratio. Steele's single pool non-steady state equations were used, to correct for small differences in glucose concentrations, to calculate glucose appearance (Ra) and glucose disposal (Rd) (33). Volume of distribution was assumed to be $0.160 \mathrm{~L} / \mathrm{kg}$ for glucose. Insulin-stimulated glucose disposal $(\Delta \mathrm{Rd})$ was calculated by the difference between $\mathrm{Rd}$ under insulin-stimulated conditions minus the $\mathrm{Rd}$ under basal non-insulin-stimulated conditions. Endogenous glucose production (EGP) was calculated as Ra minus exogenous glucose infusion rate. The percentage insulin-suppressed EGP was calculated as the percentage insulin- 
suppressed EGP from the basal EGP. Non-oxidative glucose disposal (NOGD) was calculated as $\mathrm{Rd}$ minus carbohydrate oxidation. The change in insulin stimulated NOGD ( $\triangle N O G D)$ was calculated as the NOGD during insulin minus the basal measured NOGD.

\section{Statistical analyses}

Participant characteristics are reported as mean $\pm \mathrm{SD}$, other outcome measures are reported as mean \pm SE. Data are presented for $n=12$, unless otherwise indicated. All data were evaluated for normal distribution. Differences between interventions were analyzed with a Repeated Measures ANOVA for parametric data and with a Friedman test for non-parametric data. Post hoc Bonferroni and Dunn's correction, respectively, was applied to correct for multiple testing. Statistical significance was set at $p<0.05$. Statistical analyses were performed using IBM SPSS version 23.0 for MacOS.

\section{Ethical approval}

The study was conducted in accordance with the principles of the declaration of Helsinki and approved by the Ethics Committee of the Maastricht University Medical Center. The study was registered at https://clinicaltrials.gov (NCT03912922). All participants provided written informed consent before screening.

\section{RESULTS}

\section{Participant population and study compliance}

Twelve healthy overweight and obese women (age $64 \pm 5$ years; BMI $29.2 \pm 2.9$ $\mathrm{kg} / \mathrm{m}^{2}$ ) participated and completed the study. Participants were non-smokers, had no active diseases, and had a sedentary lifestyle according to the Baecke questionnaire (6.55 \pm 0.95$)$ (Table 1). Medication use of the participants did not interfere with the main outcomes of this study (Supplementary Table 1). Habitual daily physical activity level showed an average habitual sedentary time (including sleeping time) of $17.57 \pm$ $1.15 \mathrm{~h}$, standing time $4.33 \pm 1.23 \mathrm{~h}$ and walking time $2.10 \pm 0.62 \mathrm{~h}$, with an average of $10141 \pm 3250$ steps per day (Table 1 ).

The three activity regimes were successfully implemented, as the time spent sitting, standing, walking and exercising were in accordance with the study design (Table 2). The time spent standing $(4.0 \pm 0.1 \mathrm{~h})$ and walking $(3.0 \pm 0.1 \mathrm{~h})$ were significantly higher in the SL regime compared to the SIT $(p<0.01)$ and EXE regimes $(p<0.01$, 


\section{Chapter 5}

Table 1. Participant characteristics

\begin{tabular}{|c|c|}
\hline Parameter & Mean \pm SD \\
\hline Age (years) & $64 \pm 5$ \\
\hline Body weight (kg) & $80.1 \pm 9.2$ \\
\hline BMI (kg/m2) & $29.2 \pm 2.9$ \\
\hline Fat mass (\%) & $46.1 \pm 11.3$ \\
\hline Waist circumference (cm) & $97 \pm 9$ \\
\hline Hip circumference (cm) & $110 \pm 8$ \\
\hline Fasting glucose (mmol/l) & $5.4 \pm 0.5$ \\
\hline Systolic blood pressure & $133 \pm 16$ \\
\hline Diastolic blood pressure & $85 \pm 9$ \\
\hline $\mathrm{VO}_{2} \max (\mathrm{ml} / \mathrm{min} / \mathrm{kg})$ & $23.8 \pm 4.3$ \\
\hline Physical activity level (Baecke score) & $6.55 \pm 0.95$ \\
\hline Habitual sitting + sleeping time $(\mathrm{h})$ & $17.6 \pm 1.2$ \\
\hline Habitual standing time (h) & $4.3 \pm 1.2$ \\
\hline Habitual walking time (h) & $2.1 \pm 0.6$ \\
\hline Habitual steps/day (n) & $10141 \pm 3250$ \\
\hline Habitual cadence (steps/min) & $80 \pm 5$ \\
\hline
\end{tabular}

Table 2). The times spent standing (SIT $1.3 \pm 0.1 \mathrm{~h}$, EXE $1.2 \pm 0.1 \mathrm{~h}$ ) and walking (SIT $1.0 \pm 0.0 \mathrm{~h}$, EXE $1.0 \pm 0.0 \mathrm{~h}$ ) were not significantly different between the SIT and EXE regimes ( $p=0.99$, Table 2). During the EXE regime, on average $1.0 \pm 0.1$ hours of sitting per day was substituted by cycling during a supervised exercise session at an intensity of on average $82 \pm 5$ Watt, corresponding to on average $4.60 \pm 0.15 \mathrm{MET}$. The number of steps per day were significantly higher in the SL regime $(16875 \pm 463$ steps/day) compared to the SIT $(4878 \pm 240$ steps/day, $p<0.01)$ and EXE regime (5082 \pm 165 steps/day, $p<0.01$, Table 2). Furthermore, the walking cadence was significantly higher in the SL regime $(94 \pm 2$ steps $/ \mathrm{min})$ compared to the SIT $(85 \pm 3$ steps/min, $p<0.01)$ and EXE $(86 \pm 2$ steps/min, $p<0.01)$ regimes (Table 2$)$. The estimated energy expenditure was lower in the SIT regime $(32.0 \pm 0.1 \mathrm{MET} /$ day $)$ compared to the EXE $(36.6 \pm 0.2 \mathrm{MET} /$ day, $p<0.0001)$ and SL regimes $(37.0 \pm 0.2$ MET/day, $p<0.01$, Table 2). The amount of time spent sleeping was not different between the three regimes with an average of $8.0 \pm 0.1$ hours per day $(p=0.28$, Table 2). The self-reported dietary intake was not different between the three activity regimes (data not shown). 
Table 2. Physical activity during activity regimes

\begin{tabular}{|c|c|c|c|c|c|c|c|}
\hline Parameter & SIT & EXE & SL & $p$-value & $\begin{array}{l}\text { p- } \\
\text { value } \\
\text { SIT vs } \\
\text { EXE }\end{array}$ & $\begin{array}{l}\mathrm{p}- \\
\text { value } \\
\text { SIT vs } \\
\text { SL }\end{array}$ & $\begin{array}{l}\text { p- } \\
\text { value } \\
\text { EXE vs } \\
\text { SL }\end{array}$ \\
\hline Sitting (h/day) & $13.6 \pm 0.2$ & $12.5 \pm 0.3$ & $9.2 \pm 0.2$ & $<0.01^{\star}$ & $<0.01^{\star}$ & $<0.01^{*}$ & $<0.01^{*}$ \\
\hline $\begin{array}{l}\text { Standing } \\
\text { (h/day }\end{array}$ & $1.3 \pm 0.1$ & $1.2 \pm 0.1$ & $4.0 \pm 0.1$ & $<0.01^{*}$ & 0.99 & $<0.01^{*}$ & $<0.01^{\star}$ \\
\hline Walking (h/day & $1.0 \pm 0.0$ & $1.0 \pm 0.0$ & $3.0 \pm 0.1$ & $<0.01^{\star}$ & 0.46 & $<0.01^{*}$ & $<0.01^{\star}$ \\
\hline $\begin{array}{l}\text { Exercise } \\
\text { (h/day) }\end{array}$ & - & $1.0 \pm 0.1$ & - & - & - & - & - \\
\hline $\begin{array}{l}\text { Sleeping } \\
\text { (h/day }\end{array}$ & $8.1 \pm 0.1$ & $8.1 \pm 0.1$ & $7.9 \pm 0.2$ & 0.28 & 0.99 & 0.83 & 0.29 \\
\hline Steps/day $(n)^{1}$ & $4878 \pm 240$ & $5082 \pm 165$ & $16875 \pm 463$ & $<0.01^{*}$ & 0.62 & $<0.01^{*}$ & $<0.01^{\star}$ \\
\hline 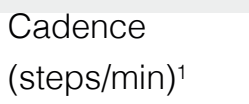 & $85 \pm 3$ & $86 \pm 2$ & $94 \pm 2$ & $<0.01^{*}$ & 0.99 & $<0.01^{\star}$ & $<0.01^{\star}$ \\
\hline $\begin{array}{l}\text { Estimated EE1 } \\
\text { (METh/day) }\end{array}$ & $32.0 \pm 0.1$ & $36.6 \pm 0.2$ & $37.0 \pm 0.2$ & $<0.01^{\star}$ & $<0.01^{*}$ & $<0.01^{*}$ & 0.38 \\
\hline
\end{tabular}

\section{Insulin sensitivity}

We examined the effects of the SIT, EXE and SL regimes on whole body and hepatic insulin sensitivity. To this end, insulin sensitivity was assessed by a two-step hyperinsulinemic-euglycemic clamp. Fasting plasma glucose levels measured before the start of the clamp were not different between the three activity regimes (SIT $5.4 \pm$ 0.1 , EXE $5.5 \pm 0.1, S L 5.4 \pm 0.1 \mathrm{mmol} / \mathrm{l}, \mathrm{p}=0.29$, Figure $2 \mathrm{~A}$ ). Whole body insulin sensitivity, as expressed by the change in glucose disposal $(\Delta R d)$, was significantly higher in the EXE $(28.2 \pm 3.3 \mu \mathrm{mol} / \mathrm{kg} / \mathrm{min}, \mathrm{p}<0.01)$ and tended to be higher in the SL regimes $(25.5 \pm 2.8 \mu \mathrm{mol} / \mathrm{kg} / \mathrm{min}, \mathrm{p}=0.08)$ compared to the SIT regime $(20.7 \pm 3.7$ $\mu \mathrm{mol} / \mathrm{kg} / \mathrm{min}$, Figure $2 \mathrm{~B}$ and Supplementary Table 2). The higher peripheral insulin sensitivity was mainly due to a significantly higher change in NODG ( $\triangle N O G D)$ in the EXE regime $(18.5 \pm 2.6 \mu \mathrm{mol} / \mathrm{kg} / \mathrm{min}, \mathrm{p}=0.01)$ and a trend towards higher NOGD in the SL regime $(15.0 \pm 2.6 \mu \mathrm{mol} / \mathrm{kg} / \mathrm{min}, \mathrm{p}=0.10)$ compared to the SIT regime $(12.2 \pm$ $2.9 \mu \mathrm{mol} / \mathrm{kg} / \mathrm{min}$, Figure $2 \mathrm{C}$ and Supplementary Table 2). Carbohydrate oxidation during high insulin stimulation remained unchanged in the three activity regimes (SIT $13.8 \pm 1.0$, EXE $13.4 \pm 1.1, \mathrm{SL} 13.7 \pm 1.2 \mu \mathrm{mol} / \mathrm{kg} / \mathrm{min}, p=0.85$ ). Hepatic insulin sensitivity, reflected by EGP suppression during the low insulin phase, was not 
affected by the three activity regimes (SIT 73.6 \pm 3.7, EXE 69.2 \pm 3.7 , SL $72.1 \pm 2.9$ $\%, p=0.98$, Figure 2D and Supplementary Table 2).
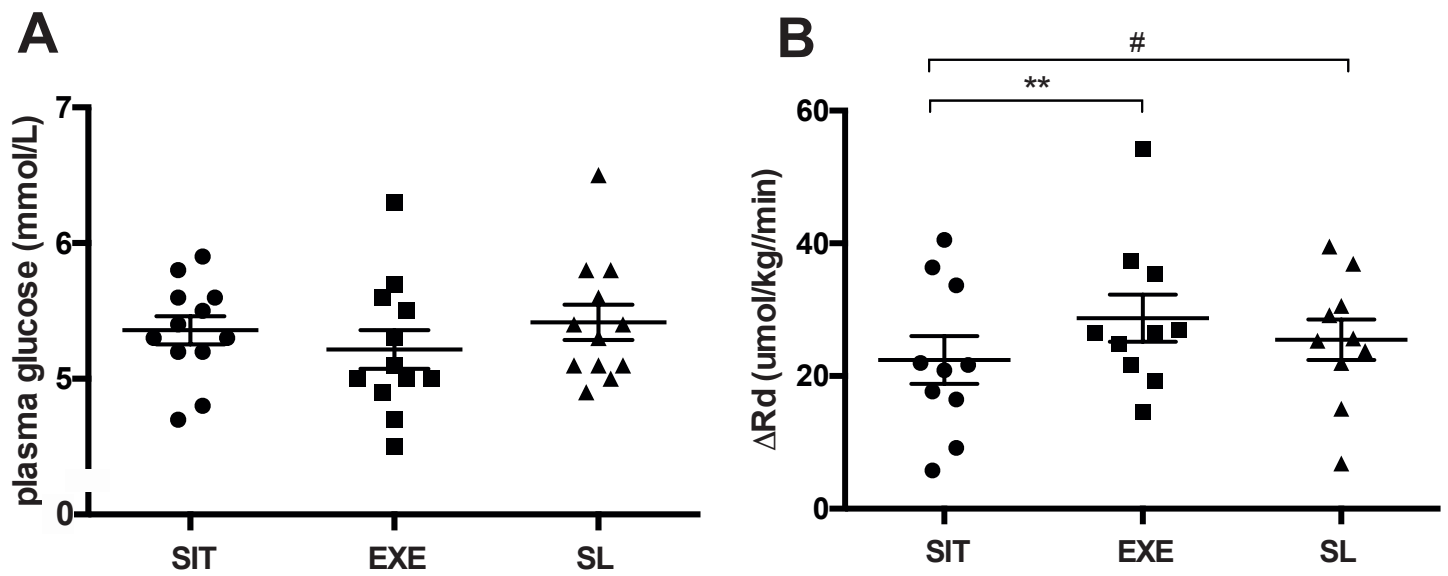

C
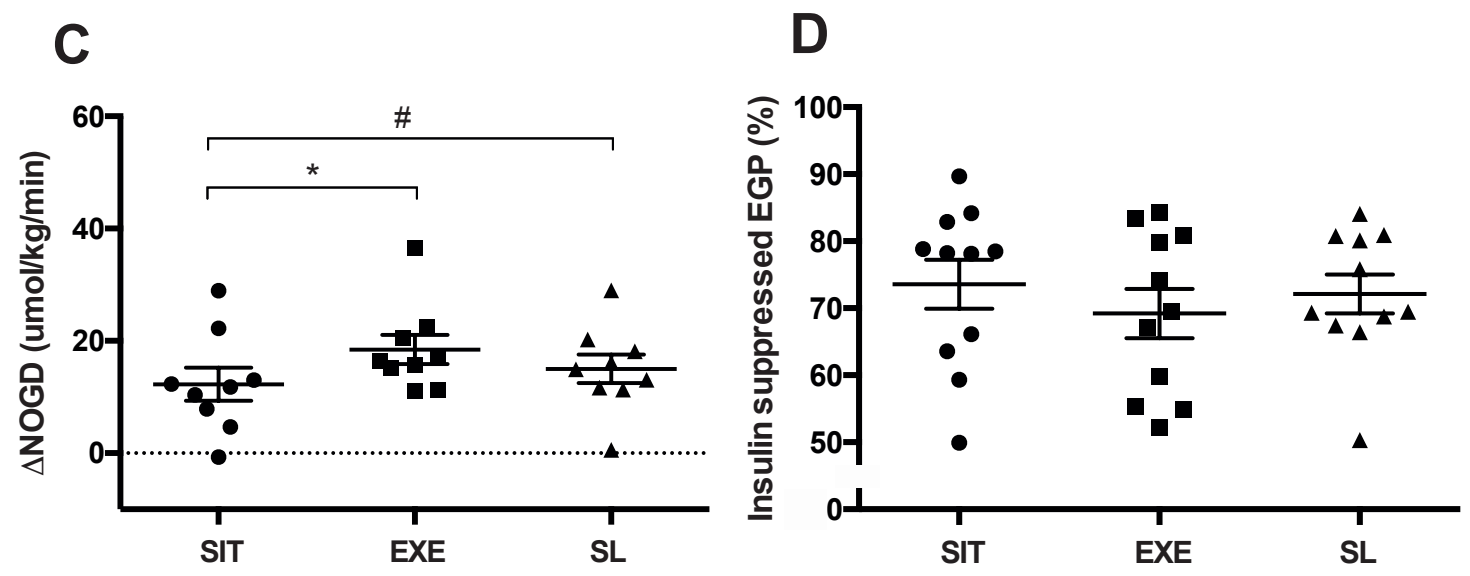

E

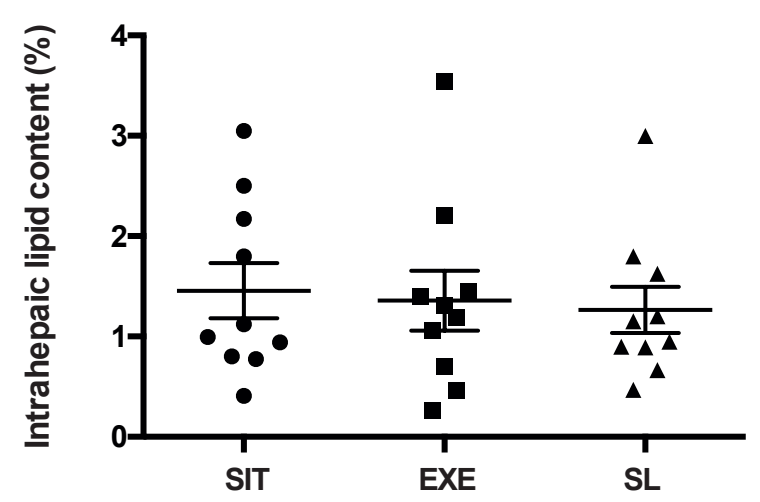

Figure 2. Insulin sensitivity and intrahepatic lipid content

Insulin sensitivity assessed by a 2-step hyperinsulinemic-euglycemic clamp. A: Fasted plasma glucose, $n=11$. B: Whole body insulin stimulated glucose disposal expressed as the delta Rd (high insulin minus baseline), $n=10$. C: Whole body non-oxidative glucose disposal expressed as the delta NOGD (high insulin minus baseline), $n=10$. D: Suppression of hepatic endogenous glucose production expressed as the delta EGP (low insulin minus baseline), $n=10$. E: Intrahepatic lipid content, $n=11$. Data are expressed as individual datapoints and mean $\pm S E$. ${ }^{*} p<0.05$ and ${ }^{\#} p<0.10$. 
Intrahepatic lipid content

Intrahepatic lipid content remained similar between the three activity regimes (SIT 1.5 \pm 0.3, EXE $1.4 \pm 0.3, S L 1.3 \pm 0.2 \%, p=0.91$, Figure 2E).

\section{Substrate kinetics}

Baseline RER tended to be lower in the SL regime compared to the SIT regime (SIT $0.78 \pm 0.01$, EXE $0.77 \pm 0.01$, SL $0.76 \pm 0.01$, SIT vs SL $p=0.10$, Figure $3 \mathrm{C}$ and Supplementary Table 2). However, fat oxidation and $\mathrm{CHO}$ oxidation were not different between regimes $(p=0.19$ and $p=0.24$ respectively, Figure 3A-B and Supplementary Table 2). In addition, fat oxidation, $\mathrm{CHO}$ oxidation and RER during insulin stimulation were not affected by the activity regimes (Supplementary Table 2).

A

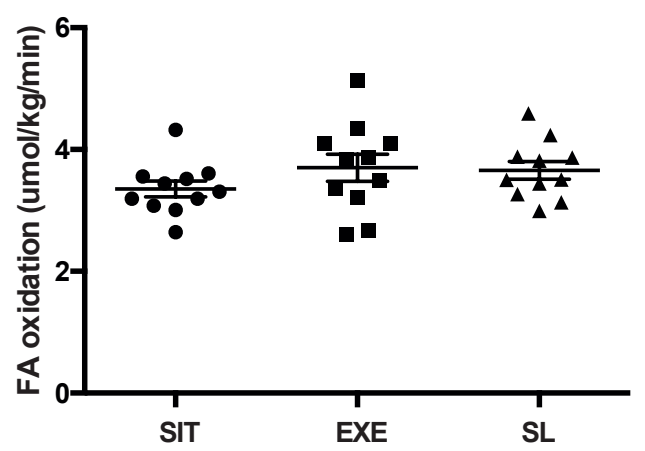

C

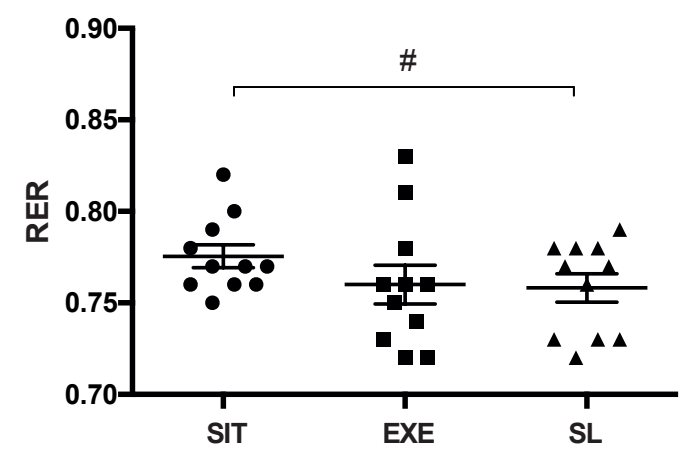

B

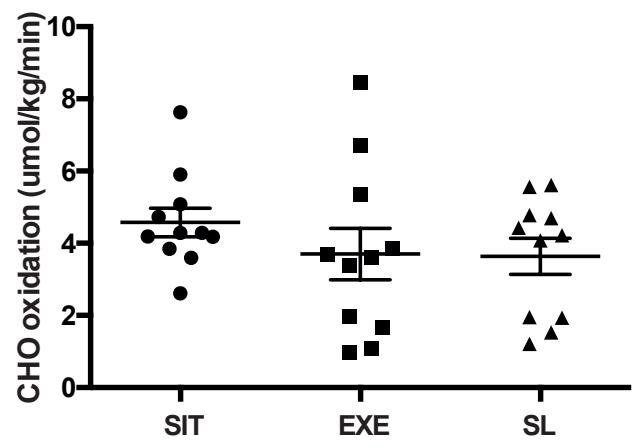

Figure 3. Baseline substrate oxidation

Baseline substrate oxidation measured by indirect calorimetry. A: Fat oxidation, $n=11$. B: Carbohydrate oxidation, $n=11$. C: Respiratory exchange ratio $n=11$. Data are expressed as individual datapoints and mean \pm SE. ${ }^{*} \mathrm{p}<0.10$ between SIT and SL regime. 


\section{Mitochondrial respiration in skeletal muscle}

We next investigated whether there would be a difference in skeletal muscle mitochondrial respiratory capacity between the three activity regimes. State 2 respiration, in the presence of substrate alone and without ATP synthesis, was not different between conditions (MO2: SIT $8.2 \pm 0.6$, EXE $8.0 \pm 0.4$, SL $8.0 \pm 0.7$ $\mathrm{pmol} / \mathrm{mg} / \mathrm{s}, \mathrm{p}=0.52$ and MG2: SIT $7.5 \pm 0.7$, EXE $7.7 \pm 0.5$, SL $6.9 \pm 0.5 \mathrm{pmol} / \mathrm{mg} / \mathrm{s}$, $\mathrm{p}=0.24$ ). Furthermore, ADP-stimulated (state 3) respiration upon complex I substrates (MG3) and upon lipid-derived substrates (MO3 and MOG3) were similar between the three activity regimes (MG3: SIT $37.5 \pm 2.3$, EXE $38.5 \pm 2.7$, SL $35.8 \pm 2.2 \mathrm{pmol} / \mathrm{mg} / \mathrm{s}$, $p=0.42$, Figure 4A, MO3: SIT 36.1 \pm 2.5 , EXE $37.7 \pm 2.2$, SL $36.2 \pm 2.9 \mathrm{pmol} / \mathrm{mg} / \mathrm{s}$, $p=0.66$, Figure 4B, MOG3: SIT 47.1 \pm 3.9 , EXE $47.5 \pm 2.6$, SL $44.7 \pm 3.3 \mathrm{pmol} / \mathrm{mg} / \mathrm{s}$, $p=0.54$, Figure $4 C$ ). Similar results were observed upon parallel electron input to both complex I and II by sequentially adding succinate in both experiments (MGS3, $p=0.25$, Figure 4D and MOGS3, $p=0.58$, Figure 4E). Maximal FCCP-induced uncoupled respiration, reflecting the maximal capacity of the electron transport chain, was also unchanged (FCCP: SIT $88.5 \pm 8.7$, EXE 93.7 \pm 5.8 , SL $87.8 \pm 7.2 \mathrm{pmol} / \mathrm{mg} / \mathrm{s}$, $\mathrm{p}=0.53$, Figure 4F). Finally, addition of oligomycin, reflecting proton leak, was similar between the three activity regimes (Oligomycin: SIT $24.5 \pm 1.8$, EXE $23.8 \pm 1.5$, SL $23.0 \pm 2.1 \mathrm{pmol} / \mathrm{mg} / \mathrm{s}, \mathrm{p}=0.52$, Figure $4 \mathrm{G}$ ).

\section{Plasma triglycerides}

Fasting plasma triglycerides were significantly lower in the EXE compared to the SIT regime (EXE 0.81 \pm 0.12 , SIT $1.01 \pm 0.15 \mathrm{mmol} / \mathrm{l}, \mathrm{p}=0.04$ ). No significant differences were observed between the SL and SIT regime (SL $0.83 \pm 0.09 \mathrm{mmol} / \mathrm{l}, p=0.13$ ) or the $S L$ and EXE regime $(p=0.99)$.

\section{Blood pressure}

Resting SBP and DBP, were not different between the three activity regimes (SBP: SIT $135 \pm 4$, EXE $138 \pm 6$, SL $135 \pm 4$ mmHg, $p=0.18$ and DBP: SIT $87 \pm 2$, EXE $87 \pm 3$, $\mathrm{SL} 87 \pm 2 \mathrm{mmHg}, \mathrm{p}=0.91)$. Resting HR was similar between the activity regimes as well (SIT: $57 \pm 1$, EXE $56 \pm 2$, SL $56 \pm 2$ bpm, $p=0.37$ ). 
A

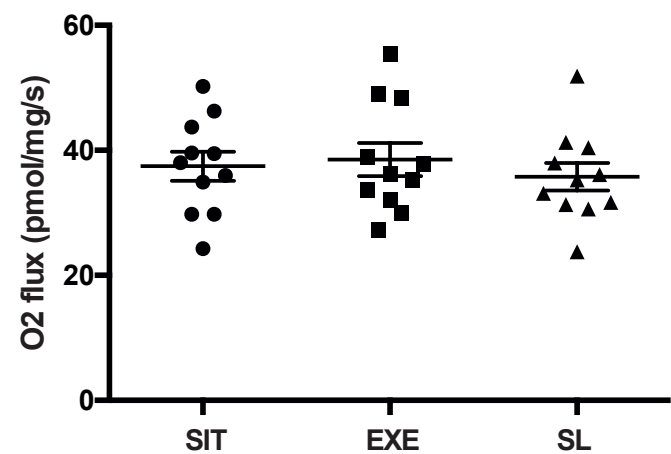

C

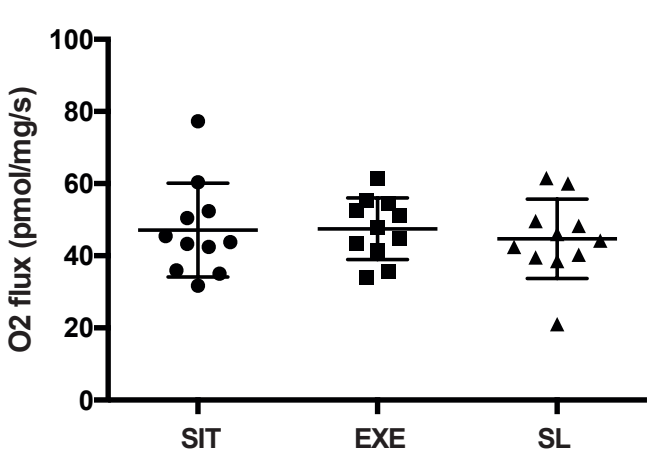

E
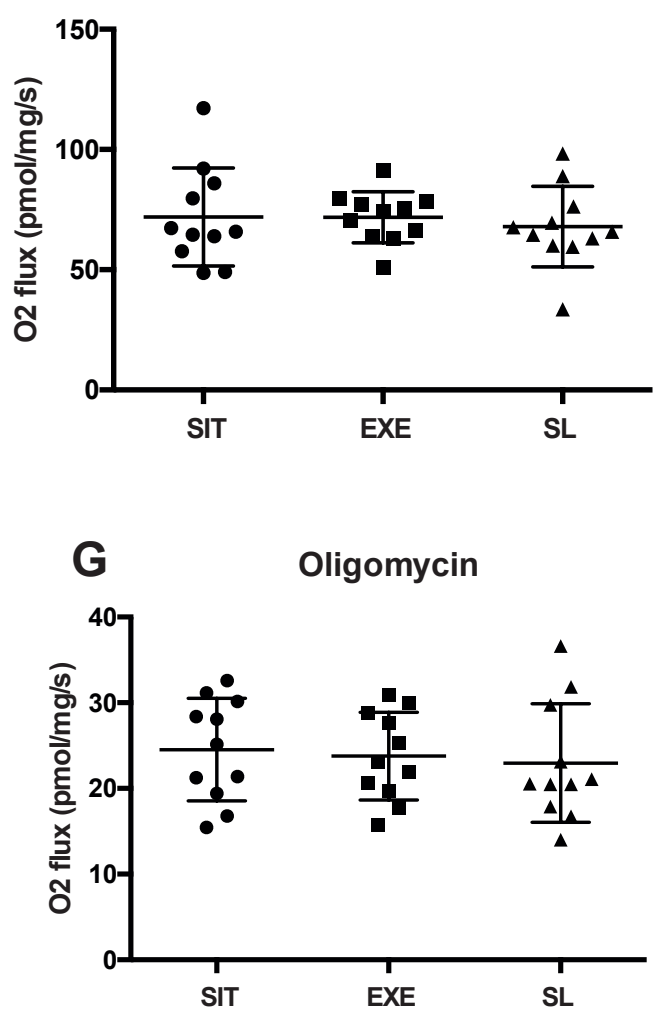

B

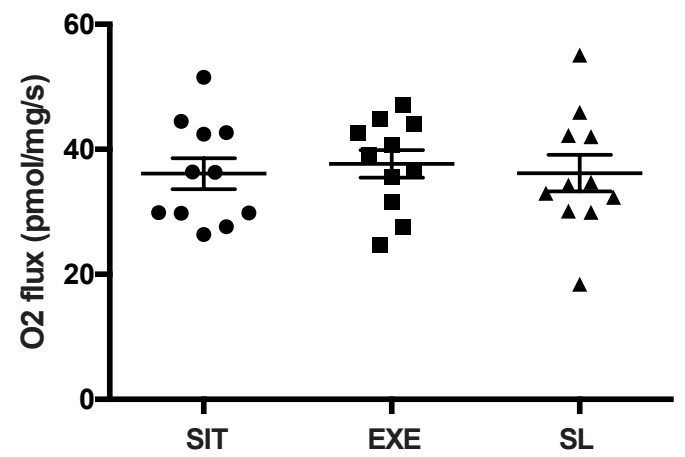

D

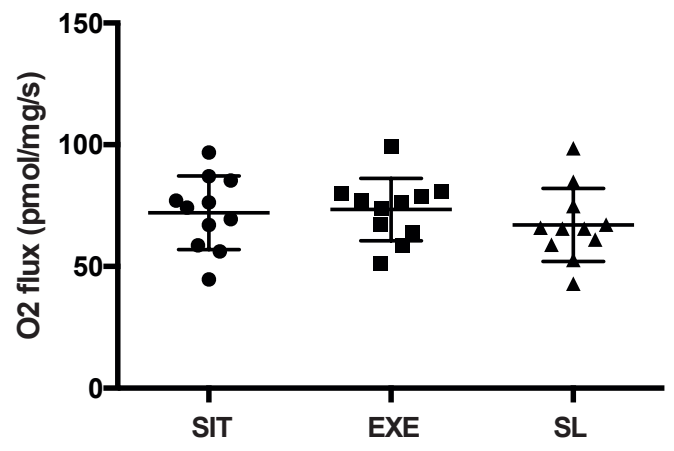

$\mathbf{F}$

FCCP

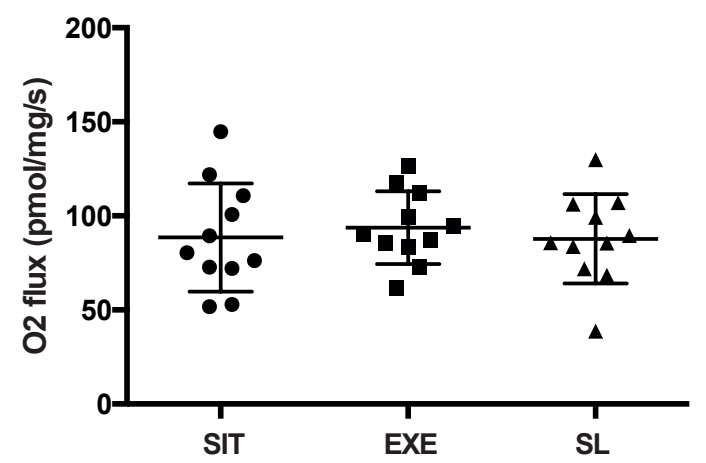

Figure 4. Ex vivo skeletal muscle mitochondrial respiratory capacity

State 3 coupled respiration upon A: malate + glutamate (MG3), B: malate + octanoyl carnitine + glutamate (MO3), C: malate + octanoyl carnitine + glutamate (MOG3), D: malate + glutamate + succinate (MGS3) and E: malate + octanoyl carnitine + glutamate + succinate (MOGS3). F: Maximal FCCP-induced uncoupled respiration. G: Oligomycin induced respiration not coupled to ATP synthesis. All $n=11$. Data are expressed as individual datapoints and mean \pm SE. 


\section{DISCUSSION}

A number of studies investigated the reduction in sedentary behaviour and showed improvements in several aspects of metabolic health, including a better glucose homeostasis (9-16). Sitting time can be replaced by light activities such as standing and walking or by moderate-to-vigorous physical activity. Both strategies have been proven effective in improving glucose homeostasis (9-16). However, the underlying mechanisms and whether these two sitting replacement strategies act via similar mechanisms remain to be investigated. Here, we hypothesized that replacing sitting time with standing and walking would exert improvements in glucose homeostasis via an increase in peripheral and hepatic insulin sensitivity. Furthermore, we hypothesized that the improvement in insulin sensitivity could coincide with an increase in muscle mitochondrial function and a reduction in intrahepatic lipid content. To investigate these hypotheses, we performed a randomized three-arm cross-over intervention study in which participants underwent three times a 4-day activity regime - sit regime, exercise regime and sit less regime - after which insulin sensitivity, muscle mitochondrial function and intrahepatic lipid content were assessed.

The activity regimes were successfully implemented as reflected by the significant differences in standing, walking, sitting and exercising time between the three activity regimes, as anticipated. By design, the exercise and sit less regimes had similar estimated daily energy expenditure. The amount of walking and standing time was kept equal in the sit and exercise regimes. This resulted in comparable sitting time between the sit and exercise regimes, and a comparable physical activity level between the sit less and exercise regimes. Thus, the intervention was successfully reproduced, as previously described by Duvivier et al. (14).

Our primary aim was to investigate the effects of the sit less and exercise regimes on insulin sensitivity compared to the sit regime. Several studies have shown improvements of reducing sedentary behaviour on glucose homeostasis measured by an oral glucose tolerance test (14-16) or by attenuated postprandial plasma glucose and insulin responses during meal tests (9-13). While oral glucose tolerance tests and meal tests give valuable information about glucose homeostasis, the gold standard for assessing human insulin sensitivity is the hyperinsulinemic-euglycemic clamp developed by DeFronzo et al (34). So far, this technique has only been used once to investigate the effects of sedentary behaviour on peripheral insulin sensitivity (35). Thus, Krogh et al. (35) applied a more extreme intervention in which healthy young participants reduced their walking activity from an habitual 10500 steps per 
day to 1400 steps per day for 2 weeks, which resulted in a decrease in peripheral insulin sensitivity (expressed as the glucose infusion rate) of $\sim 17 \%$. However, they did not compare a sit less regime with an exercise regime. Here, we applied the 2-step hyperinsulinemic-euglycemic clamp technique in combination with glucose tracer, enabling us to discriminate between peripheral and hepatic insulin sensitivity. In line with our hypothesis, we report a significant increase in peripheral insulin sensitivity with the exercise regime $(\sim 33 \%)$ and nearly significant increase with the sit less regime $(\sim 22 \%)$, both compared to the sit regime. The increase in insulin-stimulated peripheral glucose uptake could be assigned to an increase in insulin-stimulated glycogen storage (NOGD) rather than glucose oxidation. No effects of any of the regimes were detected on hepatic insulin sensitivity. Therefore, based on our results we can conclude that the sit less and the exercise regimes were both effective in improving peripheral insulin sensitivity, suggesting that reducing sitting time forms a potential effective strategy in the prevention or treatment of type 2 diabetes.

It has previously been shown by us (36) and others (37) that skeletal muscle insulin resistance is associated with a reduction in mitochondrial function. Therefore, to investigate of the beneficial effects of sit less and exercise were due to enhancement of mitochondrial capacity, we measured ex vivo skeletal muscle mitochondrial respiratory capacity upon reducing sedentary behaviour by standing and walking, and upon exercise. However, in contrast to our hypothesis, the observed improvement in peripheral insulin sensitivity could not be explained by an increase in muscle mitochondrial function. In addition, no effect on muscle mitochondrial function was found after the exercise regime either. Although it is generally accepted that exercise exerts positive effects on muscle mitochondrial function and biogenesis (19, 38 ), and that this partly underlies long term training-induced improvements seen in peripheral insulin sensitivity $(37,39)$, the duration of the current study (four days) may have been too short to detect such effects (40). Together, these data indicate that the beneficial effects of short-duration exercise or sit less regimes are independent of mitochondrial changes and other mechanisms must be underlying. To further unravel these, muscle biopsies will be used to study GLUT4 translocation and for metabolomics analyses, and these analyses are currently ongoing.

Besides an improvement in peripheral insulin sensitivity, fasted plasma triglyceride levels are affected by physical activity as well. We confirm the previously reported (14-16) reduction in fasted plasma triglyceride levels upon the exercise regime compared to the sit regime. Although not confirmed by us and some others $(9,12)$, it has in addition been shown that replacing sedentary behaviour by light intensity 
physical activities was successful as well in reducing fasted plasma triglycerides (1416). Nonalcoholic fatty liver disease is associated with increased plasma triglyceride levels (41) and high intrahepatic lipid content has shown to be an independent contributor to obesity-induced insulin resistance (42). Moreover, higher intrahepatic lipid content has been positively correlated with sedentary time in a large cohort study (43), although another study did not report such a relation (44). Therefore, we investigated whether intrahepatic lipid content was reduced upon the sit less and exercise regimes compared to the sit regime and could play a role in changes in insulin sensitivity. In contrast to our hypothesis, intrahepatic lipid content was not affected by the three activity regimes and the lack of change in intrahepatic lipid content was reflected by an absent change in hepatic insulin sensitivity. This finding contrasts the reduction in intrahepatic lipid content found upon a prolonged resistance and endurance exercise training (45), and may indicate that a four-day intervention is too short to detect alterations in intrahepatic lipid content. Furthermore, these findings indicate that the main effects of sitting less resided to peripheral insulin sensitivity. In that respect, it has been reported that a reduced triglyceride level can also be caused by enhanced clearance of triglycerides in the muscle via stimulated lipoprotein lipase activity $(46,47)$. Indeed, in rodents, it has been shown that muscle lipoprotein lipase activity is low upon inactivity and already increased upon lowintensity muscle contractions, in this case slowly walking (48). Further analysis in muscle biopsies are needed to reveal if LPL activity and triglyceride clearance in muscle was elevated upon sit less.

In conclusion, we here show that replacing sedentary time by light physical activities, such as standing and walking, is nearly as effective in improving peripheral insulin sensitivity as replacing sedentary time by a single vigorous exercise bout per day. The improvement in peripheral insulin sensitivity could however not be explained by an increase in muscle mitochondrial function. Moreover, hepatic insulin sensitivity and intrahepatic lipid content were not affected by reducing sedentary behaviour or exercise during this four-day intervention strategy. Given the more prominent role of reducing sedentary behaviour in the prevention and treatment of type 2 diabetes nowadays, future research should evaluate the practical applicability of such a sit less regime in daily life. 


\section{SUPPLEMENTARY MATERIAL FOR CHAPTER 5}

Supplementary Table 1. Medication of participants

Medication N

Lipid lowering drugs (statines)

$\mathrm{N}$

Blood pressure lowering drugs

1

Glaucoma medication

1 


\begin{tabular}{|c|c|c|c|c|c|c|c|}
\hline Parameter & SIT & EXE & SL & $p$-value & $\begin{array}{l}\mathrm{p}- \\
\text { value } \\
\text { SIT vs } \\
\text { EXE }\end{array}$ & $\begin{array}{l}\mathrm{p}- \\
\text { value } \\
\text { SIT } \\
\text { vs SL }\end{array}$ & $\begin{array}{l}\mathrm{p}- \\
\text { value } \\
\text { EXE } \\
\text { vs SL }\end{array}$ \\
\hline \multicolumn{8}{|l|}{$\mathrm{Ra}(\mu \mathrm{mol} / \mathrm{kg} / \mathrm{min})$} \\
\hline Baseline & $7.6 \pm 0.4$ & $7.3 \pm 0.5$ & $8.4 \pm 0.5$ & 0.21 & 0.99 & 0.46 & 0.31 \\
\hline Low insulin ${ }^{1}$ & $11.9 \pm 1.3$ & $11.7 \pm 1.0$ & $11.9 \pm 0.7$ & 0.73 & 0.99 & 0.99 & 0.99 \\
\hline High insulin² & $29.8 \pm 3.6$ & $36.1 \pm 3.8$ & $32.8 \pm 3.2$ & $<0.01^{* *}$ & $0.01^{*}$ & 0.22 & 0.79 \\
\hline \multicolumn{8}{|l|}{$\operatorname{Rd}(\mu \mathrm{mol} / \mathrm{kg} / \mathrm{min})$} \\
\hline Baseline & $7.3 \pm 0.4$ & $6.9 \pm 0.5$ & $7.6 \pm 0.6$ & 0.56 & 0.99 & 0.99 & 0.92 \\
\hline Low insulin ${ }^{1}$ & $12.5 \pm 1.2$ & $11.8 \pm 0.9$ & $12.3 \pm 0.9$ & 0.73 & 0.99 & 0.99 & 0.99 \\
\hline High insulin² & $29.4 \pm 3.7$ & $35.2 \pm 3.8$ & $33.1 \pm 3.2$ & $<0.01^{\star \star}$ & $<0.01^{\star \star}$ & $0.03^{*}$ & 0.99 \\
\hline $\begin{array}{l}\text { Delta baseline- } \\
\text { low }{ }^{1}\end{array}$ & $5.0 \pm 1.2$ & $4.9 \pm 0.8$ & $4.7 \pm 0.7$ & 0.98 & 0.99 & 0.99 & 0.99 \\
\hline $\begin{array}{l}\text { Delta baseline- } \\
\text { high }^{1}\end{array}$ & $20.7 \pm 3.7$ & $28.2 \pm 3.3$ & $25.5 \pm 2.8$ & $<0.01^{* *}$ & $<0.01^{* *}$ & $0.08^{\#}$ & 0.99 \\
\hline \multicolumn{8}{|l|}{$\mathrm{EGP}(\mu \mathrm{mol} / \mathrm{kg} / \mathrm{min})$} \\
\hline Baseline & $7.6 \pm 0.4$ & $7.3 \pm 0.5$ & $8.4 \pm 0.5$ & 0.21 & 0.99 & 0.46 & 0.31 \\
\hline \multirow{4}{*}{$\begin{array}{l}\text { Low insulin }{ }^{1} \\
\% \text { suppression } \\
\text { low } \\
\text { High insulin } \\
\% \text { suppression } \\
\text { high }^{2}\end{array}$} & $2.0 \pm 0.2$ & $2.3 \pm 0.2$ & $2.3 \pm 0.2$ & 0.16 & 0.60 & 0.17 & 0.99 \\
\hline & $73.6 \pm 3.7$ & $69.2 \pm 3.7$ & $72.1 \pm 2.9$ & 0.98 & 0.99 & 0.99 & 0.99 \\
\hline & $0.4 \pm 0.2$ & $0.7 \pm 0.2$ & $0.3 \pm 0.1$ & 0.71 & 0.99 & 0.99 & 0.99 \\
\hline & $93.6 \pm 2.7$ & $90.0 \pm 2.9$ & $96.4 \pm 1.9$ & 0.44 & 0.99 & 0.99 & 0.54 \\
\hline \multicolumn{8}{|c|}{ NOGD ( $\mu \mathrm{mol} / \mathrm{kg} / \mathrm{min})$} \\
\hline Baseline $^{1}$ & $2.6 \pm 0.6$ & $3.0 \pm 0.6$ & $4.0 \pm 0.7$ & 0.63 & 0.99 & 0.99 & 0.86 \\
\hline Low insulin ${ }^{1}$ & $3.9 \pm 1.2$ & $4.3 \pm 0.9$ & $4.9 \pm 1.0$ & 0.63 & 0.86 & 0.99 & 0.99 \\
\hline High insulin² & $15.7 \pm 2.8$ & $22.3 \pm 3.0$ & $19.9 \pm 2.6$ & $<0.01^{* *}$ & $<0.01^{* *}$ & $0.04^{*}$ & 0.79 \\
\hline $\begin{array}{l}\text { Delta baseline- } \\
\text { low }^{2}\end{array}$ & $1.0 \pm 1.7$ & $1.0 \pm 0.8$ & $1.0 \pm 0.7$ & 0.60 & 0.99 & 0.79 & 0.99 \\
\hline $\begin{array}{l}\text { Delta baseline- } \\
\text { high }^{3}\end{array}$ & $12.2 \pm 2.9$ & $18.5 \pm 2.6$ & $15.0 \pm 2.6$ & $0.01^{*}$ & $0.01^{*}$ & $0.10^{\#}$ & 0.99 \\
\hline \multicolumn{8}{|c|}{ Carbohydrate oxidation ( $\mu \mathrm{mol} / \mathrm{kg} / \mathrm{min})$} \\
\hline Baseline $^{1}$ & $5.0 \pm 0.6$ & $4.5 \pm 1.0$ & $4.2 \pm 0.7$ & 0.24 & 0.46 & 0.13 & 0.91 \\
\hline Low insulin ${ }^{1}$ & $8.6 \pm 0.6$ & $7.5 \pm 0.8$ & $7.5 \pm 0.6$ & 0.35 & 0.41 & 0.86 & 0.99 \\
\hline High insulin² & $13.8 \pm 1.0$ & $13.4 \pm 1.1$ & $13.7 \pm 1.2$ & 0.98 & 0.99 & 0.99 & 0.99 \\
\hline \multicolumn{8}{|c|}{ Fat oxidation ( $\mu \mathrm{mol} / \mathrm{kg} / \mathrm{min})$} \\
\hline Baseline $^{1}$ & $3.3 \pm 0.2$ & $3.7 \pm 0.2$ & $3.6 \pm 0.1$ & 0.19 & 0.33 & 0.18 & 0.81 \\
\hline Low insulin ${ }^{1}$ & $2.3 \pm 0.2$ & $2.5 \pm 0.2$ & $2.6 \pm 0.2$ & 0.26 & 0.26 & 0.99 & 0.99 \\
\hline High insulin² & $1.1 \pm 0.2$ & $1.3 \pm 0.2$ & $1.3 \pm 0.2$ & 0.51 & 0.99 & 0.72 & 0.99 \\
\hline \multicolumn{8}{|c|}{ Respiratory exchange ratio } \\
\hline Baseline $^{1}$ & $0.78 \pm 0.01$ & $0.76 \pm 0.01$ & $0.76 \pm 0.01$ & 0.16 & 0.59 & $0.10^{\#}$ & 0.99 \\
\hline Low insulin ${ }^{1}$ & $0.84 \pm 0.01$ & $0.82 \pm 0.01$ & $0.82 \pm 0.01$ & 0.20 & 0.63 & 0.22 & 0.99 \\
\hline High insulin² & $0.92 \pm 0.01$ & $0.91 \pm 0.01$ & $0.91 \pm 0.01$ & 0.63 & 0.99 & 0.99 & 0.99 \\
\hline $\begin{array}{l}\text { Delta baseline- } \\
\text { low }\end{array}$ & $0.07 \pm 0.01$ & $0.07 \pm 0.01$ & $0.06 \pm 0.01$ & 0.66 & 0.99 & 0.99 & 0.99 \\
\hline $\begin{array}{l}\text { Delta baseline- } \\
\text { high }\end{array}$ & $0.13 \pm 0.01$ & $0.14 \pm 0.02$ & $0.14 \pm 0.02$ & 0.69 & 0.99 & 0.99 & 0.99 \\
\hline
\end{tabular}

Abbreviations: SIT, sit regime; EXE, exercise regime; SL, sit less regime; Ra, rate of appearance; Rd, rate of disappearance; EGP, endogenous glucose production; NOGD, non-oxidative glucose disposal Data are expressed as mean \pm SE. ${ }^{1} n=11,{ }^{2} n=10,{ }^{3} n=9$. ${ }^{* *} p<0.01,{ }^{*} p<0.05,{ }^{\#} p<0.10$. 


\section{REFERENCES}

1. Grontved A, Hu FB. Television viewing and risk of type 2 diabetes, cardiovascular disease, and all-cause mortality: a meta-analysis. JAMA. 2011;305(23):2448-55.

2. van der Berg JD, Stehouwer CD, Bosma H, van der Velde JH, Willems PJ, Savelberg HH, et al. Associations of total amount and patterns of sedentary behaviour with type 2 diabetes and the metabolic syndrome: The Maastricht Study. Diabetologia. 2016;59(4):709-18.

3. van der Ploeg HP, Chey T, Korda RJ, Banks E, Bauman A. Sitting time and all-cause mortality risk in 222497 Australian adults. Arch Intern Med. 2012;172(6):494-500.

4. Conn VS, Koopman RJ, Ruppar TM, Phillips LJ, Mehr DR, Hafdahl AR. Insulin Sensitivity Following Exercise Interventions: Systematic Review and Meta-Analysis of Outcomes Among Healthy Adults. J Prim Care Community Health. 2014;5(3):211-22.

5. Marques A, Sarmento H, Martins J, Saboga Nunes L. Prevalence of physical activity in European adults - Compliance with the World Health Organization's physical activity guidelines. Prev Med. 2015;81:333-8.

6. Tucker JM, Welk GJ, Beyler NK. Physical activity in U.S.: adults compliance with the Physical Activity Guidelines for Americans. Am J Prev Med. 2011;40(4):454-61.

7. Morrato EH, Hill JO, Wyatt HR, Ghushchyan V, Sullivan PW. Physical activity in U.S. adults with diabetes and at risk for developing diabetes, 2003. Diabetes Care. 2007;30(2):203-9.

8. Martin A, Fitzsimons C, Jepson R, Saunders DH, van der Ploeg HP, Teixeira PJ, et al. Interventions with potential to reduce sedentary time in adults: systematic review and metaanalysis. Br J Sports Med. 2015;49(16):1056-63.

9. Peddie MC, Bone JL, Rehrer NJ, Skeaff CM, Gray AR, Perry TL. Breaking prolonged sitting reduces postprandial glycemia in healthy, normal-weight adults: a randomized crossover trial. The American journal of clinical nutrition. 2013;98(2):358-66.

10. Henson J, Davies MJ, Bodicoat DH, Edwardson CL, Gill JM, Stensel DJ, et al. Breaking Up Prolonged Sitting With Standing or Walking Attenuates the Postprandial Metabolic Response in Postmenopausal Women: A Randomized Acute Study. Diabetes care. 2016;39(1):130-8.

11. Dunstan DW, Kingwell BA, Larsen R, Healy GN, Cerin E, Hamilton MT, et al. Breaking up prolonged sitting reduces postprandial glucose and insulin responses. Diabetes care. 2012;35(5):976-83.

12. Bailey DP, Locke CD. Breaking up prolonged sitting with light-intensity walking improves postprandial glycemia, but breaking up sitting with standing does not. J Sci Med Sport. 2015;18(3):294-8.

13. Dempsey PC, Larsen RN, Sethi P, Sacre JW, Straznicky NE, Cohen ND, et al. Benefits for Type 2 Diabetes of Interrupting Prolonged Sitting With Brief Bouts of Light Walking or Simple Resistance Activities. Diabetes Care. 2016;39(6):964-72.

14. Duvivier B, Schaper NC, Koster A, van Kan L, Peters HPF, Adam JJ, et al. Benefits of Substituting Sitting with Standing and Walking in Free-Living Conditions for Cardiometabolic Risk Markers, Cognition and Mood in Overweight Adults. Front Physiol. 2017;8:353.

15. Duvivier BM, Schaper NC, Bremers MA, van Crombrugge G, Menheere PP, Kars M, et al. Minimal intensity physical activity (standing and walking) of longer duration improves insulin action and plasma lipids more than shorter periods of moderate to vigorous exercise (cycling) in sedentary subjects when energy expenditure is comparable. PloS one. 2013;8(2):e55542.

16. Duvivier BM, Schaper NC, Hesselink MK, van Kan L, Stienen N, Winkens B, et al. Breaking sitting with light activities vs structured exercise: a randomised crossover study demonstrating benefits for glycaemic control and insulin sensitivity in type 2 diabetes. Diabetologia. 2016;60(3):490-8.

17. Douen AG, Ramlal T, Rastogi S, Bilan PJ, Cartee GD, Vranic M, et al. Exercise induces recruitment of the "insulin-responsive glucose transporter". Evidence for distinct intracellular insulin- and exercise-recruitable transporter pools in skeletal muscle. J Biol Chem. 1990;265(23):13427-30.

18. Richter EA, Hargreaves M. Exercise, GLUT4, and skeletal muscle glucose uptake. Physiol Rev. 2013;93(3):993-1017.

19. Phielix E, Meex R, Moonen-Kornips E, Hesselink MK, Schrauwen P. Exercise training increases mitochondrial content and ex vivo mitochondrial function similarly in patients with type 2 diabetes and in control individuals. Diabetologia. 2010;53(8):1714-21.

20. Bergouignan A, Latouche C, Heywood S, Grace MS, Reddy-Luthmoodoo M, Natoli AK, et al. Frequent interruptions of sedentary time modulates contraction- and insulin-stimulated glucose 
uptake pathways in muscle: Ancillary analysis from randomized clinical trials. Sci Rep. 2016;6:32044.

21. Latouche C, Jowett JB, Carey AL, Bertovic DA, Owen N, Dunstan DW, et al. Effects of breaking up prolonged sitting on skeletal muscle gene expression. J Appl Physiol (1985). 2013;114(4):453-60.

22. den Hoed M, Hesselink MK, van Kranenburg GP, Westerterp KR. Habitual physical activity in daily life correlates positively with markers for mitochondrial capacity. J Appl Physiol (1985). 2008;105(2):561-8.

23. Baecke JA, Burema J, Frijters JE. A short questionnaire for the measurement of habitual physical activity in epidemiological studies. Am J Clin Nutr. 1982;36(5):936-42.

24. Dempster P, Aitkens S. A new air displacement method for the determination of human body composition. Med Sci Sports Exerc. 1995;27(12):1692-7.

25. Kuipers H, Verstappen FT, Keizer HA, Geurten P, van Kranenburg G. Variability of aerobic performance in the laboratory and its physiologic correlates. Int J Sports Med. 1985;6(4):197201.

26. Lindeboom L, Nabuurs Cl, Hesselink MK, Wildberger JE, Schrauwen P, Schrauwen-Hinderling VB. Proton magnetic resonance spectroscopy reveals increased hepatic lipid content after a single high-fat meal with no additional modulation by added protein. Am J Clin Nutr. 2015;101(1):65-71.

27. Bergstrom J, Hermansen L, Hultman E, Saltin B. Diet, muscle glycogen and physical performance. Acta Physiol Scand. 1967;71(2):140-50.

28. Hoeks J, van Herpen NA, Mensink M, Moonen-Kornips E, van Beurden D, Hesselink MK, et al. Prolonged fasting identifies skeletal muscle mitochondrial dysfunction as consequence rather than cause of human insulin resistance. Diabetes. 2010;59(9):2117-25.

29. de Ligt M, Bruls YMH, Hansen J, Habets MF, Havekes B, Nascimento EBM, et al. Resveratrol improves ex vivo mitochondrial function but does not affect insulin sensitivity or brown adipose tissue in first degree relatives of patients with type 2 diabetes. Mol Metab. 2018;12:39-47.

30. Schoffelen PFM, Plasqui G. Classical experiments in whole-body metabolism: open-circuit respirometry-diluted flow chamber, hood, or facemask systems. Eur J Appl Physiol. 2018;118(1):33-49.

31. Peronnet F, Massicotte D. Table of nonprotein respiratory quotient: an update. Can J Sport Sci. 1991;16(1):23-9.

32. Weir JB. New methods for calculating metabolic rate with special reference to protein metabolism. J Physiol. 1949;109(1-2):1-9.

33. Steele R. Influences of glucose loading and of injected insulin on hepatic glucose output. Ann N Y Acad Sci. 1959;82:420-30.

34. DeFronzo RA, Tobin JD, Andres R. Glucose clamp technique: a method for quantifying insulin secretion and resistance. Am J Physiol. 1979;237(3):E214-23.

35. Krogh-Madsen R, Thyfault JP, Broholm C, Mortensen OH, Olsen RH, Mounier R, et al. A 2-wk reduction of ambulatory activity attenuates peripheral insulin sensitivity. J Appl Physiol (1985). 2010;108(5): 1034-40.

36. Phielix E, Schrauwen-Hinderling VB, Mensink M, Lenaers E, Meex R, Hoeks J, et al. Lower intrinsic ADP-stimulated mitochondrial respiration underlies in vivo mitochondrial dysfunction in muscle of male type 2 diabetic patients. Diabetes. 2008;57(11):2943-9.

37. Toledo FG, Menshikova EV, Ritov VB, Azuma K, Radikova Z, DeLany J, et al. Effects of physical activity and weight loss on skeletal muscle mitochondria and relationship with glucose control in type 2 diabetes. Diabetes. 2007;56(8):2142-7.

38. Jornayvaz FR, Shulman Gl. Regulation of mitochondrial biogenesis. Essays Biochem. 2010;47:69-84.

39. Meex RC, Schrauwen-Hinderling VB, Moonen-Kornips E, Schaart G, Mensink M, Phielix E, et al. Restoration of muscle mitochondrial function and metabolic flexibility in type 2 diabetes by exercise training is paralleled by increased myocellular fat storage and improved insulin sensitivity. Diabetes. 2010;59(3):572-9.

40. Tonkonogi M, Sahlin K. Physical exercise and mitochondrial function in human skeletal muscle. Exerc Sport Sci Rev. 2002;30(3):129-37.

41. Tomizawa M, Kawanabe Y, Shinozaki F, Sato S, Motoyoshi Y, Sugiyama T, et al. Triglyceride is strongly associated with nonalcoholic fatty liver disease among markers of hyperlipidemia and diabetes. Biomed Rep. 2014;2(5):633-6.

42. Kusters YH, Schalkwijk CG, Houben AJ, Kooi ME, Lindeboom L, Op 't Roodt J, et al. Independent tissue contributors to obesity-associated insulin resistance. JCl Insight. 2017;2(13). 
43. Bowden Davies KA, Sprung VS, Norman JA, Thompson A, Mitchell KL, Harrold JOA, et al. Physical Activity and Sedentary Time: Association with Metabolic Health and Liver Fat. Med Sci Sports Exerc. 2019;51(6):1169-77.

44. Keating SE, Parker HM, Pavey TG, Baker MK, Caterson ID, George J, et al. Objectively Quantified Physical Activity and Sedentary Behavior in Predicting Visceral Adiposity and Liver Fat. J Obes. 2016;2016:2719014.

45. Brouwers B, Schrauwen-Hinderling VB, Jelenik T, Gemmink A, Sparks LM, Havekes B, et al. Exercise training reduces intrahepatic lipid content in people with and people without nonalcoholic fatty liver. Am J Physiol Endocrinol Metab. 2018;314(2):E165-E73.

46. Seip RL, Angelopoulos TJ, Semenkovich CF. Exercise induces human lipoprotein lipase gene expression in skeletal muscle but not adipose tissue. Am J Physiol. 1995;268(2 Pt 1):E229-36.

47. Taskinen MR, Nikkila EA. Effect of acute vigorous exercise on lipoprotein lipase activity of adipose tissue and skeletal muscle in physically active men. Artery. 1980;6(6):471-83.

48. Bey L, Hamilton MT. Suppression of skeletal muscle lipoprotein lipase activity during physical inactivity: a molecular reason to maintain daily low-intensity activity. J Physiol. 2003;551(Pt 2):673-82. 



\section{CHAPTER 6}

General discussion and conclusion

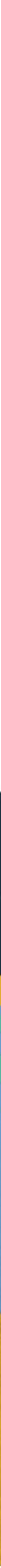



Over the last decades, the worldwide prevalence of overweight, obesity and type 2 diabetes mellitus (T2DM) has increased dramatically $(1,2)$. The fundamental cause of the increased prevalence is an unhealthy lifestyle leading to a sustained positive energy balance, in which energy intake exceeds energy expenditure for a prolonged period of time, with body mass - mostly fat mass - gain as a consequence. Obesity and excessive fat mass can lead to impaired insulin sensitivity and mitochondrial dysfunction, which are key characteristics of metabolic diseases such as T2DM. Lifestyle interventions like caloric restriction and exercise training are well known strategies to counteract this positive energy balance and to improve markers of human metabolic health, such as insulin sensitivity. The first demonstration of the beneficial health effects of dietary caloric restriction - without malnutrition - was already reported in the 1930s (3). While obesity was not a major problem at that time, caloric restriction was reported as a method for extending lifespan and slowing aging in a variety of species, while maintaining health and vitality. Further research in rodents $(4-6)$ and primates $(7,8)$ showed an improvement in glucose homeostasis reflected in an increased insulin sensitivity - and an enhanced biogenesis of mitochondria. These improvements in insulin sensitivity and mitochondrial biogenesis were considered important underlying metabolic processes in mediating the physiological health effects of caloric restriction (9). In more recent years, the effects of caloric restriction have also been studied in overweight humans in whom the health effects of a $25 \%$ reduction in energy intake for six months were evaluated (10-12). These studies observed that caloric restriction is an effective strategy to increase human insulin sensitivity and mitochondrial function, alongside improvements in other markers of metabolic health (10-12).

The effects of caloric restriction are among others mediated via downstream signals sensing a low cellular energy status, leading to activation of AMP-activated protein kinase (AMPK) and sirtuin 1 (SIRT1), and via weight loss. An alternative strategy to induce cellular energy stress is via exercise training. Exercise training is not necessarily accompanied by weight loss but does increase energy expenditure and thereby also activates downstream signals of energy stress. Exercise training involves muscle contractions, which activates translocation of the glucose transporter (GLUT4) to the muscle cell membrane to facilitate glucose uptake. Exercise-mediated translocation of GLUT4 is triggered by activation of AMPK as well. For several decades, research has been performed to study the health effects of exercise training. Beneficial effects of various exercise training programs have emerged, including improvements in glucose homeostasis, insulin sensitivity and mitochondrial function (13-18). Moreover, results conclude that exercise training is beneficial for T2DM, as it beneficially influences incidence and prognostic rates (19). 
Taken together, clear improvements in human metabolic health can be achieved via inducing a low cellular energy status by caloric restriction and via activation of skeletal muscle by exercise training. Indeed, both strategies reduce the risk of developing metabolic diseases, such as $\operatorname{T2DM}(19,20)$. Although caloric restriction and exercise training are very powerful, the practical applicability is less good; long-term compliance to a low-calorie-diet and regular exercise training is very low in the overweight and obese population who would benefit the most from these lifestyle changes $(21,22)$. Therefore, research is needed to find alternative and more readily acceptable ways to improve human metabolic health. The obtained knowledge regarding the underlying mechanisms of caloric restriction and exercise training (thus, activation of AMPK, SIRT1, and GLUT4 translocation) are useful to generate new hypothesis and to discover and design new strategies to combat metabolic diseases such as T2DM. In this thesis, the hypothesis that activation of skeletal muscle metabolism and promotion of whole-body cellular energy metabolism trigger a range of beneficial metabolic adaptations has been tested using multiple novel intervention strategies. The primary aim of this thesis was to target human insulin sensitivity and muscle mitochondrial function to improve human metabolic health.

\section{SKELETAL MUSCLE ACTIVATION: PHYSICAL ACTIVITY}

Exercise training refers to structured, planned, and repetitive physical activities with the aim to improve or maintain physical fitness (23). However, any bodily movement produced by skeletal muscles requires energy and can be described as physical activity (23). From epidemiological studies it is known that people who are more physically active display a better metabolic health, reflected by lower risk markers for T2DM and cardiovascular diseases, than people who are less physically active (19, 24-26). Importantly, high physical activity levels have been associated with a reduced mortality risk $(19,27)$. In recent years, not only the amount of time spent exercising, but also the amount of time spent sedentary has been associated with T2DM, the metabolic syndrome $(28,29)$, and increased mortality risk (30). A recently published review highlighted that physical inactivity - including short-term inactivity - induces peripheral insulin resistance in skeletal muscle and adipose tissue with consequent accumulation of fat in the liver, leading to hepatic insulin resistance (31). Fortunately, it has also been suggested that the effects of physical inactivity are reversible (31). Therefore, research has been performed on different strategies to reduce sedentary behaviour.

Sedentary time can be replaced by moderate-to-vigorous physical activities such as exercise training or by light intensity physical activities such as standing and walking. Whether sedentary time should be replaced by exercise training or by standing and 
walking to obtain maximal benefit, is still under debate. Several human intervention studies in which sedentary time was replaced by standing $(32,33)$, walking (32-36) or short exercise bouts (36) have all shown improvements in glucose homeostasis. In general, standing and walking is more feasible for a large proportion of the obese population for whom exercise compliance generally is low. Therefore, it needs to be investigated whether these sitting replacement strategies possess beneficial health effects. It has previously been observed that a four-day intervention, in which replacement of sedentary time by 3 hours of standing and 2 hours of walking, or by a single 1-hour exercise-bout per day, both improved glucose and lipid metabolism, including markers for whole-body insulin sensitivity (37-39). In three different studies, these effects were reported in healthy young adults, healthy obese men and women and in patients with T2DM (37-39). Furthermore, the effects were more pronounced in women (37). Results indicate that sedentary time indeed can be replaced by light intensity physical activities such as standing and walking to improve metabolic health parameters. The question which remains to be answered is whether these two sitting replacement strategies (standing/walking vs. exercise) act via similar or different underlying pathways involving skeletal muscle.

In chapter 5, we show that replacing sedentary time by several hours of standing and walking (sit less regime) was similar effective in improving human peripheral - mainly skeletal muscle - insulin sensitivity compared to a single moderate-to-vigorous exercise bout per day (exercise regime) in healthy obese women. These results are in agreement with previous studies which reported improvements in markers of wholebody insulin sensitivity after similar interventions, measured by an oral glucose tolerance test and by 24-hour glucose monitoring (37-39). We examined insulin sensitivity by a 2-step hyperinsulinemic euglycemic clamp combined with a glucose tracer, which is the gold standard to assess peripheral and hepatic insulin sensitivity (40). We show that hepatic insulin sensitivity and also hepatic lipid content were not affected by the four-day intervention (chapter 5), which means that merely muscle was responsible for the exerted improved insulin-stimulated glucose uptake.

Improvements in insulin sensitivity after exercise training are reported together with an increased muscle mitochondrial function and muscle mitochondrial content (15). Therefore, we collected muscle tissue in our study and measured muscle mitochondrial respiratory capacity to investigate whether the beneficial effects of the four-day sit less regime and the four-day exercise regime on peripheral insulin sensitivity involved improvements in muscle mitochondrial function. However, we found that neither the sit less nor the exercise regime affected skeletal muscle mitochondrial function compared to the sit regime (chapter 5). Acute exercise can 
also increase insulin sensitivity independent of effects on mitochondrial function or hepatic metabolism. As explained previously, acute exercise activates pathways in skeletal muscle in response to changes in energy status. This in turn leads to activation of AMPK and subsequent GLUT4 translocation, which stimulates glucose uptake in muscle and increases insulin sensitivity (41). These effects remain several hours after the final exercise-bout and involve enhanced glucose uptake in the liver to replenish glycogen stores (41). Therefore, our results suggest that short-term improvements in peripheral insulin sensitivity induced by standing and walking probably act via similar pathways in skeletal muscle as acute exercise training. It has, however, been shown that longer term exercise training interventions do report positive effects regarding muscle mitochondrial function and hepatic lipid content after for example 12 weeks of training $(14,16)$. Thus, we cannot rule out that longerterm interventions in which sedentary time is replaced by standing and walking can also improve muscle mitochondrial function and hepatic metabolism. In the near future, muscle tissue sampled in the present study will be studied using transcriptomics and metabolomics to identify early signs of metabolic adaptations following the sit less regime in comparison to the exercise regime.

\section{SKELETAL MUSCLE ACTIVATION: SHIVERING}

An alternative way to increase energy expenditure is by cold exposure. Physiological responses to cold exposure include insulative responses by peripheral vasoconstriction and metabolic responses by increasing energy expenditure with the aim to produce heat and to maintain core body temperature stable. Heat is a side product of energy expenditure, both when work is performed, but also when energy is produced to fuel vital bodily functions. This thermogenic metabolic response can be divided into shivering thermogenesis and non-shivering thermogenesis. Shivering thermogenesis is a physiological reaction of the human body to acute cold exposure and includes involuntary muscle contractions. Non-shivering thermogenesis increases metabolic rate without activating muscle shivering, and brown adipose tissue (BAT) plays a major role herein. It has been reported that before the onset of shivering, metabolic rate already increases indicating that non-shivering thermogenesis precedes shivering (42). BAT is a special form of adipose tissue, characterized by a large number of mitochondria that contain the uncoupling protein UCP1, which puts BAT mitochondria in the position to generate heat rather than ATP. BAT-based non-shivering thermogenesis has an evolutionary advantage during times when heat loss is massive, such as in newborn and small mammals, who are prone to hypothermia due to their high surface-to-body ratio. The relevance of BAT in adult humans has long been questioned, since the absolute amount (and hence its capacity for thermogenesis) is considered to be very low in adult humans. However, 
it has been shown that BAT activity and thermogenesis is negatively correlated with body fat mass in humans (43). Therefore, a relationship was proposed between the increase in the prevalence of obesity and the fact that many people nowadays live in their thermoneutral zone for most of the day, thus, there is no need to increase energy expenditure for thermal comfort (44). Therefore, re-activating BAT is a way to promote energy expenditure and thereby could contribute to changes in body mass.

In the last decade, BAT has received much scientific attention as the major side of non-shivering thermogenesis, and it has been investigated whether increasing BAT activity or volume could protect against obesity and related metabolic diseases such as T2DM. Indeed, studies in adult humans have shown that acute cold exposure activates BAT and increases whole body energy expenditure (45-48). In chapter 3 we confirm that acute mild cold exposure just below the individual shivering threshold increases BAT activity and resting energy expenditure in healthy obese humans.

Interestingly, in recent years it has been reported several times that cold exposure may also affect insulin sensitivity $(46,49-51)$ and that these effects may be in the same order of magnitude as exercise training (51). Originally, activation of BAT was the proposed mechanism of action. More recently, however, it has been shown that cold acclimation mainly affects peripheral insulin sensitivity via an effect on skeletal muscle GLUT4 translocation (51). Indeed, both skeletal muscle $(52,53)$ and BAT $(43,47,54$, 55) contribute to non-shivering thermogenesis during cold acclimation. Muscle-based non-shivering thermogenesis is energetically costly (56) and thereby can contribute to a higher energy expenditure as well. Considering that more than $40 \%$ of total body mass is represented by skeletal muscle, and that multiple non-shivering thermogenesis mechanisms have been identified within skeletal muscle (mitochondrial uncoupling, protein turnover, fatty acid cycling, and calcium cycling (57)), it is capable of producing a significant amount of heat and thereby contributing to whole body thermogenesis. However, although it is suggested that muscle-based non-shivering thermogenesis might be a target to improve metabolic health (51), part of the effects of cold acclimation on insulin sensitivity may also be explained by shivering thermogenesis.

When skin and core temperature decrease, shivering intensity increases $(58,59)$. Heat production through shivering thermogenesis can reach maximal values equivalent to around five times resting metabolic rate (60). In comparison, high intensity exercise can increase resting metabolic rate up to 20 times (61). Interestingly, upon acute cold exposure to $5^{\circ} \mathrm{C}$ in humans, fat oxidation and carbohydrate oxidation increase to mobilize fuels for thermogenesis (62). Moreover, 
acute cold exposure to $10^{\circ} \mathrm{C}$ in humans may promote insulin sensitivity, an effect that predominantly originates from muscles that actually are shivering (63). These data suggest that part of the effect of cold exposure on insulin sensitivity could be due to a stimulation of shivering thermogenesis.

Previous research performed within our department reported an increase of approximately $40 \%$ in peripheral insulin sensitivity upon ten days of mild cold acclimation $\left(14-15^{\circ} \mathrm{C}\right)(51)$. The reported increase in peripheral insulin sensitivity was accompanied by increased translocation of GLUT4 to the cell membrane in skeletal muscle. Given the relative mild cold exposure and the attempt to minimize shivering, it was suggested that this improvement in insulin sensitivity was due to a combination of non-shivering thermogenesis in BAT and skeletal muscle (51). To further investigate this, in chapter 4, we performed a study with a similar study design (ten days of mild cold acclimation in overweight and obese patients with T2DM), except with a slightly higher temperature $\left(16-17^{\circ} \mathrm{C}\right)$ and with more detailed attention for the prevention of shivering. The aim of the study was to investigate whether the cold-induced improvements in peripheral insulin sensitivity (as observed before (51)) were sustained for a longer period of time after cessation of the cold intervention, and whether this was accompanied by improvements in postprandial glucose and lipid metabolism and markers of cardiovascular health. Unexpectedly, we could not reproduce the improvements in peripheral insulin sensitivity upon mild cold exposure and hence also no improvements in the other outcomes were observed (chapter 4). Given the focus on non-shivering thermogenesis and the somewhat higher temperature during cold exposure in our study, we investigated whether the two studies could be distinguished from each other by the presence or absence of shivering. Comparison of questionnaire results showed that some mild form of shivering occurred in the previous study (51) compared to almost no shivering in our current study (chapter 4). Subsequently, analyses in skeletal muscle biopsies were performed to evaluate GLUT4 translocation and gene expression of markers for skeletal muscle contraction. In contrast to the previous study (51), we did not show any translocation of GLUT4 to the cell membrane after the cold acclimation, matching with the absent improvement in peripheral insulin sensitivity in our current study (chapter 4). In addition, we performed gene-expression analysis in skeletal muscle biopsies obtained before and after cold acclimation in the previous and current study. Genes were selected from micro-array data obtained after the previous cold acclimation study (51) compared to an exercise training study (14), to function as markers of muscle contraction. The selected genes revealed overlap in skeletal muscle gene expression-patterns related to muscle contraction pathways 
(unpublished data). Thus, these muscle contraction markers were increased in the previous study, suggesting that shivering may have occurred in the previous study (51). Interestingly, no change in skeletal muscle gene expression was observed in the current study (chapter 4). Therefore, our results indicate that modest shivering or increased muscle tension may be the underlying pathway through which improvements in peripheral insulin sensitivity and GLUT4 translocation were observed upon ten days mild cold acclimation in the previous study (51), but not in our current study (chapter 4). Further research is needed to quantify the amount of shivering that is needed to induce metabolic changes, which can for example be done by electromyographic activity measurements. Also, metabolic pathways that may be activated by this mild shivering, such as AMPK activation, need to be studied to understand how mild shivering is connected to an improvement in skeletal muscle insulin sensitivity.

\section{PROMOTING CELLULAR ENERGY AND NAD+ METABOLISM}

Besides the investigation of several strategies to promote skeletal muscle activity, another strategy is to search for compounds that can mimic the beneficial effects of caloric restriction by promoting energy metabolism without the need to change lifestyle. To be effective, a caloric restriction mimetic should alter the key metabolic pathways. Thus, targeting SIRT1 activation either directly or indirectly via AMPK may result in similar beneficial effects as observed after caloric restriction. Several caloric restriction mimetics have been studied in the past years. The oral antidiabetic drug metformin is the most described one and is thought to mediate its anti-hyperglycemia and insulin-sensitizing effects via AMPK and SIRT1 activation (64, 65). Another example is the nutritional compound resveratrol, which activates AMPK and SIRT1 $(66,67)$ and has been reported to improve human skeletal muscle mitochondrial capacity after 30 days supplementation $(66,68,69)$. Furthermore, resveratrol and other polyphenols, such as quercetin, reduce reactive oxygen species production and increase mitochondrial capacity (70). Mitochondrial capacity can be improved by enhancing mitochondrial biogenesis, but also by enhancing mitophagy and thus improving mitochondrial quality via increasing mitochondrial turnover. Urolithin $A$ is an example of a nutritional compound that stimulates mitophagy and has recently been shown to induce a molecular signature in human skeletal muscle of improved mitochondrial and cellular health (71). These examples show that several ways of targeting SIRT1 activity and cellular energy metabolism have been studied and can be used to stimulate mitochondrial metabolism in humans.

One key metabolite in the signaling of cellular energy status that may link stimulation of energy metabolism to improvement in mitochondrial function and metabolic health 
is nicotinamide adenine dinucleotide $\left(\mathrm{NAD}^{+}\right) . \mathrm{NAD}^{+}$is a critical signaling molecule of cellular energy status and is an essential substrate for the $\mathrm{NAD}^{+}$-dependent enzyme SIRT1. As previously explained, SIRT1 is a metabolic regulator of oxidative energy metabolism and is activated in situations of energy stress like exercise and caloric restriction (72). Furthermore, caloric restriction and exercise training are known to upregulate the enzyme nicotinamide phosphoribosyl transferase (NAMPT), which is involved in $\mathrm{NAD}^{+}$synthesis $(73,74)$. NAMPT levels correlate positively with skeletal muscle mitochondrial function and overall aerobic capacity (73). Since NAD+ levels are decreased in the obese and older population (75-77), replenishment of $\mathrm{NAD}^{+}$ levels is hypothesized to be a new strategy to improve human metabolic health, and is suggested to have more profound effects compared to other nutritional compounds such as resveratrol. NAD+ levels can be increased via dietary supplementation of $\mathrm{NAD}^{+}$-precursors or via inhibition of $\mathrm{NAD}^{+}$degradation pathways, or both.

In humans, several NAD+ precursors have already been tested. Thus, nicotinic acid and acipimox are effective in lowering circulating lipids, but due to a rebound effect, fatty acids in the circulation increase on the longer term with increased muscle lipid content, insulin resistance, and elevated plasma glucose levels as consequences (78, 79). Nicotinamide is another factor that has been shown to increase NAD+ levels, however, nicotinamide has not been reported to have beneficial metabolic health effects so far, and high dosages are hepatotoxic (80). However, all these three NAD+precursors, have been shown to induce unwarranted side effects in humans by binding to the G protein-coupled receptor, GPR109A, and thereby inducing flushing $(81,82)$. Nicotinamide mononucleotide (NMN) and nicotinamide riboside (NR) second generation $\mathrm{NAD}^{+}$-precursors - do not induce these side effects and are orally bioavailable endogenous molecules. This renders NMN and NR the molecules of choice for human trials nowadays. In this thesis, the effects of NR on human metabolic health has been investigated.

So far, $\mathrm{NAD}^{+}$replenishment by nutritional supplementation with NR has been successful in improving insulin sensitivity and other parameters of metabolic health in animal models $(75,83-86)$. Within these animal studies, it has been shown that NR is taken up in several tissues including skeletal muscle, liver, heart and brown adipose tissue, reflected by increased levels of $\mathrm{NAD}^{+}$within these tissues (83). Whether NR is also taken up in white adipose tissue is still inconclusive $(83,86)$. Clinical trials in humans are warranted to test the translational value of the results from this animal research. Therefore, in this thesis, the effects of six weeks NR supplementation in healthy overweight and obese humans have been investigated on whole body energy metabolism and on specific tissues. In chapter 2 we show that upon NR 
supplementation, markers of enhanced $\mathrm{NAD}^{+}$metabolism in skeletal muscle are induced. No change in skeletal muscle NAD+ content was detected, however. Although inconsistent with animal data on NR supplementation $(83,84)$, our results are in agreement with other data from human intervention studies that investigated the effects of NR supplementation on skeletal muscle NAD+ metabolism and reported increased NAD+ metabolites (NAAD and MeNAM) as well $(87,88)$. Furthermore, in our and previous studies, no positive effects on skeletal muscle mitochondrial respiration, peripheral insulin sensitivity or intramyocellular lipid content were observed (87-89) (chapter 2).

These results indicate that NR does not have the hypothesized effects on human skeletal muscle metabolism. Furthermore, our results indicate that skeletal muscle $\mathrm{NAD}^{+}$turnover is increased, which is not reflected in steady state $\mathrm{NAD}^{+}$levels in skeletal muscle. Results in chapter 2 however support the idea that NR indeed may have modest effects on human skeletal muscle, as an increase in fat free mass which mainly represents muscle mass - upon NR supplementation was observed. The increase in muscle mass might help to understand the observed increase in sleeping metabolic rate after NR supplementation. Moreover, skeletal muscle acetylcarnitine levels were affected by NR supplementation. The role of acetylcarnitines in regulating i.e. fatty acid oxidation and the role of NR herein needs further investigation. Future studies should be designed to reproduce if NR supplementation can indeed increase muscle mass and affect acetylcarnitine levels in humans and to investigate the underlying mechanisms. Besides these modest effects of NR on human skeletal muscle metabolism, no other metabolic effects were observed on whole body substrate oxidation (chapter 2) or in other tissues, such as intrahepatic lipid content, cardiac energy status (chapter 2) or brown adipose tissue activity (chapter 3 ). While, we were successful in reproducing the earlier reported (83) stimulatory effects of NR in high fat diet-fed mice on BAT activity in chapter 3 , again no effects of NR on human BAT in vivo were shown (chapter 3 ). Further research is needed to evaluate various dosages and long-term supplementation to investigate if $\mathrm{NR}$ can have effects on human metabolic health.

\section{CONCLUDING REMARKS AND FUTURE PERSPECTIVES}

Research described in this thesis aimed to investigate novel strategies to improve human metabolic health with the main focus on insulin sensitivity and muscle mitochondrial function. Over the past decades it has become clear that caloric restriction and exercise training are successful strategies to improve human metabolic health, including insulin sensitivity and mitochondrial function, and that this leads to a reduction in the risk on developing T2DM and cardiovascular diseases. It 
has been confirmed in this thesis that physical activity is a powerful tool to increase peripheral insulin sensitivity, already after a four-day intervention. Moderate-tovigorous physical activities such as exercise, as well as light-intensity physical activities such as standing and walking, promoted peripheral insulin sensitivity to a similar extent in healthy obese women (chapter 5). The aim of this four-day intervention study was to investigate whether similar pathways were responsible for this effect. So far, similar metabolic routes were observed between the two sitting replacement strategies in outcomes regarding peripheral and hepatic insulin sensitivity, muscle mitochondrial function, and hepatic lipid content. Completion of this study by performing analysis in the obtained skeletal muscle tissue should reveal more information about underlying molecular pathways activated by standing and walking versus exercise. Pathways of interest to measure include GLUT4 translocation, insulin signaling, and calcium cycling since these pathways play a role in insulin sensitivity and cellular energy status. Furthermore, the determination of triglyceride clearance, lipoprotein lipase activity, and intramyocellular lipid storage would be of interest as well, since we (chapter 5) and others (32, 33, 39, 90) report lower triglyceride levels after short-term exercise training, and some studies also after a standing and walking intervention $(37-39,91)$. The long-term effects of a sit less regime should be investigated, as this could shed more light on the underlying pathways in skeletal muscle. Lastly, dose-response studies should be performed to evaluate the optimal intensity, duration and pattern of sedentary interruptions to provide information for public recommendations.

From another point of view, physical activity in the form of shivering might be a successful strategy to increase peripheral insulin sensitivity as well. Results presented in chapter 4 provide evidence that shivering thermogenesis might be an important underlying mechanism through which improvements in peripheral insulin sensitivity after ten days of mild cold exposure have been reported previously (51). Although acute mild cold exposure without shivering is able to increase whole body energy metabolism via BAT activation in healthy obese humans (chapter 3 ), this cold stimulus does not seem to be powerful enough to also induce changes in skeletal muscle metabolism of patients with T2DM after 10 days of mild cold acclimation (chapter 4). Future studies should investigate underlying metabolic pathways that may be activated by (mild) shivering - for example AMPK activation - to understand how shivering is linked to improvements in skeletal muscle insulin sensitivity. Furthermore, the minimal intensity and duration of shivering that exerts sustained beneficial metabolic health effects must be identified.

Our third novel strategy was to improve human metabolic health by promoting NAD+ metabolism. Results from this thesis illustrate that there is limited evidence that six 
weeks of supplementation with the $\mathrm{NAD}^{+}$precursor $\mathrm{NR}$ is effective in improving markers of metabolic health in healthy obese humans (chapter 2 and 3). The results obtained in our study and by other human clinical trials (87-89) do not support the hypothesis that NR supplementation is beneficial in improving muscle mitochondrial function and insulin sensitivity in humans. However, it does not mean that further research is not warranted. Interestingly, we and others $(87,88)$ do show some effects of $\mathrm{NR}$ supplementation on elevating $\mathrm{NAD}^{+}$metabolite levels in skeletal muscle and in addition we show some minor effects in skeletal muscle metabolism regarding fat free mass and acetylcarnitine levels (chapter 2). Further research is needed to investigate the exact link between NR metabolism and acetylcarnitine metabolism. Furthermore, as we and others only investigated the effects of NR supplementation in a middleaged, obese, healthy population, we cannot exclude the possibility that NR would have effects in other populations, for example those at risk for T2DM or patients diagnosed with T2DM. In addition, the large gap between metabolic improvements upon NR observed in mice and the absence of effects in humans may originate from the fact that mice studies applied a much longer supplementation period (up to 15 weeks) - especially when this supplementation period is considered relative to the lifespan - compared to short-term human clinical trials (maximum of 12 weeks). Another possibility might be a lower tissue bioavailability of oral NR supplementation in humans compared to mice. More research should be done to unravel the exact pharmacokinetic properties of NR in humans and the possible differences between mice and humans. A recently identified new NAD+-precursor, a reduced form of NR called $\mathrm{NRH}$, acts via a slightly different metabolic pathway and has been shown to be more potent in increasing $\mathrm{NAD}^{+}$levels in mice tissue compared to NR (92). For NRH, the next step would be to evaluate safety and tolerability of $\mathrm{NRH}$ in humans and eventually investigate whether NRH might have the hypothesized effects on human metabolic health which has not been shown so far upon NR. 


\section{REFERENCES}

1. WHO. Factsheet obesity and overweight 2018 [Available from: https://www.who.int/newsroom/fact-sheets/detail/obesity-and-overweight.

2. WHO. Factsheet diabetes 2018 [Available from: https://www.who.int/news-room/factsheets/detail/diabetes.

3. McCay CM, Crowell MF, Maynard LA. The effect of retarded growth upon the length of life span and upon the ultimate body size. 1935. Nutrition (Burbank, Los Angeles County, Calif). 1989;5(3):155-71; discussion 72.

4. Kalant N, Stewart J, Kaplan R. Effect of diet restriction on glucose metabolism and insulin responsiveness in aging rats. Mech Ageing Dev. 1988;46(1-3):89-104.

5. Lambert AJ, Wang B, Yardley J, Edwards J, Merry BJ. The effect of aging and caloric restriction on mitochondrial protein density and oxygen consumption. Experimental gerontology. 2004;39(3):289-95.

6. Nisoli E, Tonello C, Cardile A, Cozzi V, Bracale R, Tedesco L, et al. Calorie restriction promotes mitochondrial biogenesis by inducing the expression of eNOS. Science (New York, NY). 2005;310(5746):314-7.

7. Bodkin NL, Ortmeyer HK, Hansen BC. Long-term dietary restriction in older-aged rhesus monkeys: effects on insulin resistance. J Gerontol A Biol Sci Med Sci. 1995;50(3):B142-7.

8. Lane MA, Ingram DK, Roth GS. Beyond the rodent model: Calorie restriction in rhesus monkeys. Age (Omaha). 1997;20(1):45-56.

9. Roth GS, Ingram DK, Black A, Lane MA. Effects of reduced energy intake on the biology of aging: the primate model. Eur J Clin Nutr. 2000;54 Suppl 3:S15-20.

10. Heilbronn LK, de Jonge L, Frisard MI, DeLany JP, Larson-Meyer DE, Rood J, et al. Effect of 6month calorie restriction on biomarkers of longevity, metabolic adaptation, and oxidative stress in overweight individuals: a randomized controlled trial. Jama. 2006;295(13):1539-48.

11. Civitarese AE, Carling S, Heilbronn LK, Hulver MH, Ukropcova B, Deutsch WA, et al. Calorie restriction increases muscle mitochondrial biogenesis in healthy humans. PLoS Med. 2007;4(3):e76.

12. Larson-Meyer DE, Heilbronn LK, Redman LM, Newcomer BR, Frisard MI, Anton S, et al. Effect of calorie restriction with or without exercise on insulin sensitivity, beta-cell function, fat cell size, and ectopic lipid in overweight subjects. Diabetes care. 2006;29(6):1337-44.

13. Toledo FG, Menshikova EV, Ritov VB, Azuma K, Radikova Z, DeLany J, et al. Effects of physical activity and weight loss on skeletal muscle mitochondria and relationship with glucose control in type 2 diabetes. Diabetes. 2007;56(8):2142-7.

14. Meex RC, Schrauwen-Hinderling VB, Moonen-Kornips E, Schaart G, Mensink M, Phielix E, et al. Restoration of muscle mitochondrial function and metabolic flexibility in type 2 diabetes by exercise training is paralleled by increased myocellular fat storage and improved insulin sensitivity. Diabetes. 2010;59(3):572-9.

15. Phielix E, Meex R, Moonen-Kornips E, Hesselink MK, Schrauwen P. Exercise training increases mitochondrial content and ex vivo mitochondrial function similarly in patients with type 2 diabetes and in control individuals. Diabetologia. 2010;53(8):1714-21.

16. Brouwers B, Schrauwen-Hinderling VB, Jelenik T, Gemmink A, Sparks LM, Havekes B, et al. Exercise training reduces intrahepatic lipid content in people with and people without nonalcoholic fatty liver. Am J Physiol Endocrinol Metab. 2018;314(2):E165-E73.

17. Pesta D, Hoppel F, Macek C, Messner H, Faulhaber M, Kobel C, et al. Similar qualitative and quantitative changes of mitochondrial respiration following strength and endurance training in normoxia and hypoxia in sedentary humans. Am J Physiol Regul Integr Comp Physiol. 2011;301(4):R1078-87.

18. Sogaard D, Lund MT, Scheuer CM, Dehlbaek MS, Dideriksen SG, Abildskov CV, et al. Highintensity interval training improves insulin sensitivity in older individuals. Acta Physiol (Oxf). 2018;222(4):e13009.

19. Yerramalla MS, Fayosse A, Dugravot A, Tabak AG, Kivimaki M, Singh-Manoux A, et al. Association of moderate and vigorous physical activity with incidence of type 2 diabetes and subsequent mortality: 27 year follow-up of the Whitehall II study. Diabetologia. 2019.

20. Xue B, Kahn BB. AMPK integrates nutrient and hormonal signals to regulate food intake and energy balance through effects in the hypothalamus and peripheral tissues. The Journal of physiology. 2006;574(Pt 1):73-83.

21. Morrato EH, Hill JO, Wyatt HR, Ghushchyan V, Sullivan PW. Physical activity in U.S. adults with diabetes and at risk for developing diabetes, 2003. Diabetes care. 2007;30(2):203-9. 
22. Gibson AA, Sainsbury A. Strategies to Improve Adherence to Dietary Weight Loss Interventions in Research and Real-World Settings. Behav Sci (Basel). 2017;7(3).

23. Caspersen CJ, Powell KE, Christenson GM. Physical activity, exercise, and physical fitness: definitions and distinctions for health-related research. Public Health Rep. 1985;100(2):126-31.

24. Dunstan DW, Salmon J, Owen N, Armstrong T, Zimmet PZ, Welborn TA, et al. Associations of TV viewing and physical activity with the metabolic syndrome in Australian adults. Diabetologia. 2005;48(11):2254-61.

25. Healy GN, Wijndaele K, Dunstan DW, Shaw JE, Salmon J, Zimmet PZ, et al. Objectively measured sedentary time, physical activity, and metabolic risk: the Australian Diabetes, Obesity and Lifestyle Study (AusDiab). Diabetes care. 2008;31(2):369-71.

26. JHPM VDV, Koster A, JD VDB, Sep SJS, CJH VDK, Dagnelie PC, et al. Sedentary Behavior, Physical Activity, and Fitness-The Maastricht Study. Med Sci Sports Exerc. 2017;49(8):158391.

27. Ekelund U, Steene-Johannessen J, Brown WJ, Fagerland MW, Owen N, Powell KE, et al. Does physical activity attenuate, or even eliminate, the detrimental association of sitting time with mortality? A harmonised meta-analysis of data from more than 1 million men and women. Lancet. 2016;388(10051):1302-10.

28. JD VDB, JHPM VDV, EAC DEW, Bosma H, Savelberg H, Schaper NC, et al. Replacement Effects of Sedentary Time on Metabolic Outcomes: The Maastricht Study. Med Sci Sports Exerc. 2017;49(7):1351-8.

29. van der Berg JD, Stehouwer CD, Bosma H, van der Velde JH, Willems PJ, Savelberg HH, et al. Associations of total amount and patterns of sedentary behaviour with type 2 diabetes and the metabolic syndrome: The Maastricht Study. Diabetologia. 2016;59(4):709-18.

30. van der Ploeg HP, Chey T, Korda RJ, Banks E, Bauman A. Sitting time and all-cause mortality risk in 222497 Australian adults. Arch Intern Med. 2012;172(6):494-500.

31. Bowden Davies KA, Pickles S, Sprung VS, Kemp GJ, Alam U, Moore DR, et al. Reduced physical activity in young and older adults: metabolic and musculoskeletal implications. Ther Adv Endocrinol Metab. 2019;10:2042018819888824.

32. Peddie MC, Bone JL, Rehrer NJ, Skeaff CM, Gray AR, Perry TL. Breaking prolonged sitting reduces postprandial glycemia in healthy, normal-weight adults: a randomized crossover trial. The American journal of clinical nutrition. 2013;98(2):358-66.

33. Henson J, Davies MJ, Bodicoat DH, Edwardson CL, Gill JM, Stensel DJ, et al. Breaking Up Prolonged Sitting With Standing or Walking Attenuates the Postprandial Metabolic Response in Postmenopausal Women: A Randomized Acute Study. Diabetes care. 2016;39(1):130-8.

34. Dunstan DW, Kingwell BA, Larsen R, Healy GN, Cerin E, Hamilton MT, et al. Breaking up prolonged sitting reduces postprandial glucose and insulin responses. Diabetes care. 2012;35(5):976-83.

35. Bailey DP, Locke CD. Breaking up prolonged sitting with light-intensity walking improves postprandial glycemia, but breaking up sitting with standing does not. J Sci Med Sport. 2015;18(3):294-8.

36. Dempsey PC, Sacre JW, Larsen RN, Straznicky NE, Sethi P, Cohen ND, et al. Interrupting prolonged sitting with brief bouts of light walking or simple resistance activities reduces resting blood pressure and plasma noradrenaline in type 2 diabetes. J Hypertens. 2016;34(12):237682.

37. Duvivier B, Schaper NC, Koster A, van Kan L, Peters HPF, Adam JJ, et al. Benefits of Substituting Sitting with Standing and Walking in Free-Living Conditions for Cardiometabolic Risk Markers, Cognition and Mood in Overweight Adults. Front Physiol. 2017;8:353.

38. Duvivier BM, Schaper NC, Bremers MA, van Crombrugge G, Menheere PP, Kars M, et al. Minimal intensity physical activity (standing and walking) of longer duration improves insulin action and plasma lipids more than shorter periods of moderate to vigorous exercise (cycling) in sedentary subjects when energy expenditure is comparable. PloS one. 2013;8(2):e55542.

39. Duvivier BM, Schaper NC, Hesselink MK, van Kan L, Stienen N, Winkens B, et al. Breaking sitting with light activities vs structured exercise: a randomised crossover study demonstrating benefits for glycaemic control and insulin sensitivity in type 2 diabetes. Diabetologia. 2016;60(3):490-8.

40. DeFronzo RA, Tobin JD, Andres R. Glucose clamp technique: a method for quantifying insulin secretion and resistance. Am J Physiol. 1979;237(3):E214-23.

41. Richter EA, Hargreaves M. Exercise, GLUT4, and skeletal muscle glucose uptake. Physiol Rev. 2013;93(3):993-1017. 
42. van Ooijen AM, van Marken Lichtenbelt WD, van Steenhoven AA, Westerterp KR. Seasonal changes in metabolic and temperature responses to cold air in humans. Physiology \& behavior. 2004;82(2-3):545-53.

43. Hanssen MJ, van der Lans AA, Brans B, Hoeks J, Jardon KM, Schaart G, et al. Short-term Cold Acclimation Recruits Brown Adipose Tissue in Obese Humans. Diabetes. 2016;65(5):1179-89.

44. Keith SW, Redden DT, Katzmarzyk PT, Boggiano MM, Hanlon EC, Benca RM, et al. Putative contributors to the secular increase in obesity: exploring the roads less traveled. Int J Obes (Lond). 2006;30(11):1585-94.

45. van Marken Lichtenbelt WD, Schrauwen P, van De Kerckhove S, Westerterp-Plantenga MS. Individual variation in body temperature and energy expenditure in response to mild cold. Am J Physiol Endocrinol Metab. 2002;282(5):E1077-83.

46. Chondronikola M, Volpi E, Borsheim E, Porter C, Annamalai P, Enerback S, et al. Brown adipose tissue improves whole-body glucose homeostasis and insulin sensitivity in humans. Diabetes. 2014;63(12):4089-99.

47. van der Lans AA, Hoeks J, Brans B, Vijgen GH, Visser MG, Vosselman MJ, et al. Cold acclimation recruits human brown fat and increases nonshivering thermogenesis. The Journal of clinical investigation. 2013;123(8):3395-403.

48. Yoneshiro T, Aita S, Matsushita M, Kayahara T, Kameya T, Kawai Y, et al. Recruited brown adipose tissue as an antiobesity agent in humans. The Journal of clinical investigation. 2013;123(8):3404-8.

49. Iwen KA, Backhaus J, Cassens M, Waltl M, Hedesan OC, Merkel M, et al. Cold-Induced Brown Adipose Tissue Activity Alters Plasma Fatty Acids and Improves Glucose Metabolism in Men. J Clin Endocrinol Metab. 2017;102(11):4226-34.

50. Lee P, Smith S, Linderman J, Courville AB, Brychta RJ, Dieckmann W, et al. Temperatureacclimated brown adipose tissue modulates insulin sensitivity in humans. Diabetes. 2014;63(11):3686-98.

51. Hanssen MJ, Hoeks J, Brans B, van der Lans AA, Schaart G, van den Driessche JJ, et al. Shortterm cold acclimation improves insulin sensitivity in patients with type 2 diabetes mellitus. Nat Med. 2015;21(8):863-5.

52. Wijers SLJ, Schrauwen P, Saris WHM, Lichtenbelt WDV. Human Skeletal Muscle Mitochondrial Uncoupling Is Associated with Cold Induced Adaptive Thermogenesis. PloS one. 2008;3(3).

53. Blondin DP, Daoud A, Taylor T, Tingelstad HC, Bezaire V, Richard D, et al. Four-week cold acclimation in adult humans shifts uncoupling thermogenesis from skeletal muscles to brown adipose tissue. The Journal of physiology. 2017;595(6):2099-113.

54. van Marken Lichtenbelt WD, Vanhommerig JW, Smulders NM, Drossaerts JM, Kemerink GJ, Bouvy ND, et al. Cold-activated brown adipose tissue in healthy men. $\mathrm{N}$ Engl J Med. 2009;360(15):1500-8.

55. Blondin DP, Labbe SM, Tingelstad HC, Noll C, Kunach M, Phoenix S, et al. Increased brown adipose tissue oxidative capacity in cold-acclimated humans. J Clin Endocrinol Metab. 2014;99(3):E438-46.

56. Bal NC, Maurya SK, Singh S, Wehrens XH, Periasamy M. Increased Reliance on Muscle-based Thermogenesis upon Acute Minimization of Brown Adipose Tissue Function. The Journal of biological chemistry. 2016;291(33):17247-57.

57. Wijers SL, Saris WH, van Marken Lichtenbelt WD. Recent advances in adaptive thermogenesis: potential implications for the treatment of obesity. Obes Rev. 2009;10(2):218-26.

58. Jansky L, Janakova H, Ulicny B, Sramek P, Hosek V, Heller J, et al. Changes in thermal homeostasis in humans due to repeated cold water immersions. Pflugers Arch. 1996;432(3):368-72.

59. Tikuisis P, Bell DG, Jacobs I. Shivering onset, metabolic response, and convective heat transfer during cold air exposure. J Appl Physiol (1985). 1991;70(5):1996-2002.

60. Eyolfson DA, Tikuisis P, Xu X, Weseen G, Giesbrecht GG. Measurement and prediction of peak shivering intensity in humans. Eur J Appl Physiol. 2001;84(1-2):100-6.

61. Ainsworth BE, Haskell WL, Herrmann SD, Meckes N, Bassett DR, Jr., Tudor-Locke C, et al. 2011 Compendium of Physical Activities: a second update of codes and MET values. Med Sci Sports Exerc. 2011;43(8):1575-81.

62. Vallerand AL, Zamecnik J, Jones PJ, Jacobs I. Cold stress increases lipolysis, FFA Ra and TG/FFA cycling in humans. Aviat Space Environ Med. 1999;70(1):42-50.

63. Vallerand AL, Frim J, Kavanagh MF. Plasma glucose and insulin responses to oral and intravenous glucose in cold-exposed humans. J Appl Physiol (1985). 1988;65(6):2395-9.

64. Klip A, Leiter LA. Cellular mechanism of action of metformin. Diabetes care. 1990;13(6):696704. 
65. Cuyàs E, Verdura S, Llorach-Parés L, Fernádez-Arroyo S, Joven J, Martin-Castillo B, et al. Metformin is a direct SIRT1-activating compound: computational modeling and experimental validation. Front Endocrinol. 2018.

66. Timmers S, de Ligt M, Phielix E, van de Weijer T, Hansen J, Moonen-Kornips E, et al. Resveratrol as Add-on Therapy in Subjects With Well-Controlled Type 2 Diabetes: A Randomized Controlled Trial. Diabetes care. 2016;39:2211-7.

67. Park SJ, Ahmad F, Philp A, Baar K, Williams T, Luo H, et al. Resveratrol ameliorates agingrelated metabolic phenotypes by inhibiting cAMP phosphodiesterases. Cell. 2012;148(3):42133.

68. Timmers S, Konings E, Bilet L, Houtkooper RH, van de Weijer T, Goossens GH, et al. Calorie restriction-like effects of 30 days of resveratrol (resVida) supplementation on energy metabolism and metabolic profile in obese humans. Cell metabolism. 2011;14(5).

69. de Ligt M, Bruls YMH, Hansen J, Habets MF, Havekes B, Nascimento EBM, et al. Resveratrol improves ex vivo mitochondrial function but does not affect insulin sensitivity or brown adipose tissue in first degree relatives of patients with type 2 diabetes. Mol Metab. 2018;12:39-47.

70. Wood Dos Santos T, Cristina Pereira Q, Teixeira L, Gambero A, J AV, Lima Ribeiro M. Effects of Polyphenols on Thermogenesis and Mitochondrial Biogenesis. Int J Mol Sci. 2018;19(9).

71. Andreux PA, Blanco-Bose W, Ryu D, Burdet F, Ibberson M, P. A, et al. The mitophagy activator urolithin $\mathrm{A}$ is safe and induces a molecular signature of improved mitochondrial and cellular health in humans. Nat Metab. 2019;1(6):595-603.

72. Canto C, Gerhart-Hines Z, Feige JN, Lagouge M, Noriega L, Milne JC, et al. AMPK regulates energy expenditure by modulating NAD+ metabolism and SIRT1 activity. Nature. 2009;458(7241):1056-60.

73. Costford SR, Bajpeyi S, Pasarica M, Albarado DC, Thomas SC, Xie H, et al. Skeletal muscle NAMPT is induced by exercise in humans. Am J Physiol Endocrinol Metab. 2010;298(1):E11726.

74. Rappou E, Jukarainen S, Rinnankoski-Tuikka R, Kaye S, Heinonen S, Hakkarainen A, et al. Weight Loss Is Associated With Increased NAD(+)/SIRT1 Expression But Reduced PARP Activity in White Adipose Tissue. J Clin Endocrinol Metab. 2016;101(3):1263-73.

75. Yoshino J, Mills KF, Yoon MJ, Imai S. Nicotinamide mononucleotide, a key NAD(+) intermediate, treats the pathophysiology of diet- and age-induced diabetes in mice. Cell metabolism. 2011;14(4):528-36.

76. Massudi H, Grant R, Braidy N, Guest J, Farnsworth B, Guillemin GJ. Age-associated changes in oxidative stress and NAD+ metabolism in human tissue. PloS one. 2012;7(7):e42357.

77. de Guia RM, Agerholm M, Nielsen TS, Consitt LA, Sogaard D, Helge JW, et al. Aerobic and resistance exercise training reverses age-dependent decline in $\mathrm{NAD}(+)$ salvage capacity in human skeletal muscle. Physiol Rep. 2019;7(12):e14139.

78. Poynten AM, Gan SK, Kriketos AD, O'Sullivan A, Kelly JJ, Ellis BA, et al. Nicotinic acid-induced insulin resistance is related to increased circulating fatty acids and fat oxidation but not muscle lipid content. Metabolism. 2003;52(6):699-704.

79. van de Weijer T, Phielix E, Bilet L, Williams EG, Ropelle ER, Bierwagen A, et al. Evidence for a direct effect of the nad+ precursor acipimox on muscle mitochondrial function in humans. Diabetes. 2015;64:1193-201.

80. Winter SL, Boyer JL. Hepatic toxicity from large doses of vitamin B3 (nicotinamide). N Engl J Med. 1973;289(22):1180-2.

81. Benyo Z, Gille A, Kero J, Csiky M, Suchankova MC, Nusing RM, et al. GPR109A (PUMA$\mathrm{G} / \mathrm{HM} 74 \mathrm{~A})$ mediates nicotinic acid-induced flushing. The Journal of clinical investigation. 2005;115(12):3634-40.

82. Connell $\mathrm{NJ}$, Houtkooper $\mathrm{RH}$, Schrauwen P. NAD(+) metabolism as a target for metabolic health: have we found the silver bullet? Diabetologia. 2019;62(6):888-99.

83. Canto C, Houtkooper RH, Pirinen E, Youn DY, Oosterveer MH, Cen Y, et al. The NAD(+) precursor nicotinamide riboside enhances oxidative metabolism and protects against high-fat diet-induced obesity. Cell metabolism. 2012;15(6):838-47.

84. Khan NA, Auranen M, Paetau I, Pirinen E, Euro L, Forsstrom S, et al. Effective treatment of mitochondrial myopathy by nicotinamide riboside, a vitamin B3. EMBO molecular medicine. 2014;6(6):721-31.

85. Trammell SA, Weidemann BJ, Chadda A, Yorek MS, Holmes A, Coppey LJ, et al. Nicotinamide Riboside Opposes Type 2 Diabetes and Neuropathy in Mice. Sci Rep. 2016;6:26933.

86. Shi W, Hegeman MA, van Dartel DAM, Tang J, Suarez M, Swarts H, et al. Effects of a wide range of dietary nicotinamide riboside (NR) concentrations on metabolic flexibility and white adipose tissue (WAT) of mice fed a mildly obesogenic diet. Mol Nutr Food Res. 2017;61(8). 
87. Dollerup OL, Chubanava S, Agerholm M, Søndergard SD, Altintas A, Møller AB, et al. Nicotinamide riboside does not alter mitochondrial respiration, content or morphology in skeletal muscle from obese and insulin resistant men. The Journal of physiology. 2019.

88. Elhassan YS, Kluckova K, Fletcher RS, Schmidt MS, Garten A, Doig CL, et al. Nicotinamide Riboside Augments the Aged Human Skeletal Muscle NAD(+) Metabolome and Induces Transcriptomic and Anti-inflammatory Signatures. Cell reports. 2019;28(7):1717-28 e6.

89. Dollerup OL, Christensen B, Svart M, Schmidt MS, Sulek K, Ringgaard S, et al. A randomized placebo-controlled clinical trial of nicotinamide riboside in obese men: safety, insulinsensitivity, and lipid-mobilizing effects. The American journal of clinical nutrition. 2018;108(2):343-53.

90. Dempsey PC, Larsen RN, Sethi P, Sacre JW, Straznicky NE, Cohen ND, et al. Benefits for Type 2 Diabetes of Interrupting Prolonged Sitting With Brief Bouts of Light Walking or Simple Resistance Activities. Diabetes care. 2016;39(6):964-72.

91. Duvivier B, Bolijn JE, Koster A, Schalkwijk CG, Savelberg H, Schaper NC. Reducing sitting time versus adding exercise: differential effects on biomarkers of endothelial dysfunction and metabolic risk. Sci Rep. 2018;8(1):8657.

92. Giroud-Gerbetant J, Joffraud M, Giner MP, Cercillieux A, Bartova S, Makarov MV, et al. A reduced form of nicotinamide riboside defines a new path for $N A D(+)$ biosynthesis and acts as an orally bioavailable NAD(+) precursor. Mol Metab. 2019;30:192-202. 




\section{APPENDICES}

Valorization

Summary

Samenvatting

Dankwoord

List of publications About the author

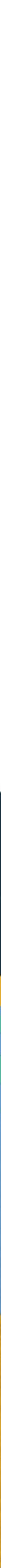





\section{VALORIZATION}

\section{SOCIAL RELEVANCE}

Obesity is a worldwide increasing problem, warranting for new prevention and treatment strategies to reduce the risk on developing chronic diseases related to excess body weight. According to the 2016 report of the World Health Organization (WHO) the prevalence of obesity is still increasing worldwide, with $39 \%$ of adults aged 18 years and older being overweight ( $>1.9$ billion adults) and $13 \%$ being obese ( $>600$ million adults) (1). It is especially alarming that 41 million children under the age of 5 , and 340 million children and adolescents aged 5-19 were overweight and obese in 2016 (1). Overweight and obesity are major risk factors for the development of type 2 diabetes mellitus (T2DM). Just like obesity, the prevalence of T2DM has been steadily increasing over the past few decades. T2DM is associated with various comorbidities, including cardiovascular diseases and non-alcoholic fatty liver disease as well as an increased mortality risk. The WHO underscores in their global report on diabetes the enormous scale of the diabetes problem, and also the potential to reverse current trends (2). It has also been pointed out by the WHO that obesity is preventable (1). Furthermore, T2DM is one of the four priority noncommunicable diseases (NCDs) targeted by world leaders in the 2011 Political Declaration on the Prevention and Control of NCDs (2). These reports and declarations highlight the importance of the prevention and treatment of obesity and T2DM worldwide.

The fundamental causes of obesity and T2DM include a high caloric diet and insufficient physical activity. Effective tools are available to prevent obesity and T2DM and to improve disease management to reduce the risk of complications. Promoting physical activity is considered one of the most potent strategies to prevent and treat T2DM. World leaders agreed that one of the global targets for the prevention of T2DM (and other NCDs) to be attained by 2025 is a 10\% relative reduction in the prevalence of insufficient physical activity (2). Other effective strategies for the prevention and treatment of obesity and T2DM include a healthy diet low in calories. Existing strategies that stimulate physical activity and promote a healthy diet are effective in reducing body weight and improving glucose homeostasis in patients with obesity and T2DM. However, these strategies seem to be challenging to adhere to on the long term for the people at risk. Therefore, it is important to investigate other strategies to prevent and treat obesity and T2DM. The present thesis describes three novel approaches that could lead to novel prevention and treatment strategies for people with obesity and obesity-related comorbidities such as T2DM and cardiovascular diseases. 
Supplementation with the NAD+ precursor nicotinamide riboside has been investigated in this thesis as the first novel approach. Nicotinamide riboside can be easily added to the normal diet, which would enhance the compliance for the general public in contrast to low compliance rates for exercise training or a rigorous diet. Although it has been shown that nicotinamide riboside had many metabolic benefits including improvements in insulin sensitivity in animal models, these effects could so far not be translated to humans, as also shown in this thesis. However, since a relative short intervention period was applied in this thesis, future research should investigate whether long term supplementation with nicotinamide riboside could have metabolic benefits in humans to prevent or treat obesity and T2DM. The second novel approach that was investigated in this thesis was non-shivering cold acclimation. From this thesis we conclude that non-shivering cold acclimation for ten days at $16-17^{\circ} \mathrm{C}$ does not have major beneficial health effects in humans with T2DM. However, it could be suggested that mild shivering induced by cold environmental temperatures, either indoor or outdoor, could be a novel way to improve metabolic health for the general public. More research is needed to quantify the amount and intensity of shivering that is needed to contribute to the prevention and treatment of obesity and T2DM, and the practical applicability in daily life. As a third novel approach we investigated whether replacing sitting time with low-intensity physical activities would have the same metabolic benefits compared to replacing sitting time with a single moderate-tovigorous exercise bout per day. In this thesis we demonstrated that low-intensity physical activities such as standing and walking are similar effective in improving insulin sensitivity compared to a single moderate-to-vigorous exercise bout per day. Taken together, investigating novel approaches that have beneficial health effects and that can be implemented in daily life are of great social relevance to prevent and treat obesity, T2DM and other metabolic diseases.

\section{ECONOMIC RELEVANCE}

Obesity, T2DM and related comorbidities are a major economic burden for national health care systems, as well as for the patients and their families. In the Netherlands, total health care costs for T2DM (including comorbidities) were 1.6 billion euro's in 2017 (3). Preventing obesity and T2DM via inexpensive and easy to access strategies such as the ones presented in this thesis can contribute to a reduction in global economic burden. Increasing physical activity by standing and walking is not only freely accessible and inexpensive for the general public, it does also contribute to a reduction in transportation use and costs. Food supplements, such as nicotinamide riboside, can be an inexpensive and easy addition to a healthy diet. Furthermore, lowering indoor temperatures and thereby challenging the body to deal with cooler environments, cannot only could improve human health but can also could reduce 
heating costs of homes, offices and buildings. Above all, a reduction in transportation and heating contributes to less emission of greenhouse gasses. Scientific evidence exists that warming of the climate system is unequivocal and that greenhouse gasses emitted by human activities are the primary driver (4). The Paris Agreement signed in 2016 by 195 countries, states that by 2050 the emission of greenhouse gasses has to be reduced by 95\% in comparison to the level in $1990(5,6)$. As obesity and T2DM is an increasing problem and at the same time climate change is an urgent matter, the investigation of novel health promoting strategies to prevent and treat obesity and T2DM that are at the same time climate-friendly are of great economic relevance.

\section{ACTIVITIES AND PRODUCTS}

All studies described within this thesis were executed at the Department of Nutrition and Movement Sciences at Maastricht University within the Diabetes and Metabolism Research Group. The Diabetes and Metabolism Research Group investigates the mechanisms underlying the pathogenesis of T2DM and related metabolic diseases by performing translational research. The studies presented in this thesis were performed in close collaboration with the Department of Radiology and Nuclear Medicine of Maastricht University Medical Centre. This collaboration between scientists and the clinic allowed us to use non-invasive techniques like MR and a PETCT scanning. Furthermore, research presented in this thesis is part of a large consortium (CVON ENERGISE 2014-02) between Amsterdam Medical Center, Leiden University Medical Center, Wageningen University and Maastricht University. Being part of such a consortium enhances collaborations between the different centers and thereby improves translational research.

The results presented in this thesis are or will be published in original scientific articles in international well-recognized peer-reviewed journals. Importantly, articles will be available online and can be assessed by scientists or other interested people worldwide. Furthermore, results from the studies described in this thesis are communicated and presented to the scientific community on (inter)national conferences and symposia via oral presentations and posters. Via this way, research knowledge was spread within the scientific community to facilitate new research ideas and hypotheses and to promote future research.

\section{INNOVATION}

The study results presented in this thesis are obtained in humans by state-of-the-art methods and techniques. When human clinical trials are performed, it is of great importance that reliable results are obtained in the most non-invasive way possible. In this thesis, we applied relatively new MRS techniques developed by our research 


\section{Appendices}

group to examine among others lipid accumulation in the muscle and muscle acetylcarnitine dynamics in a non-invasive way. In the past, these outcomes could only be studied and quantified with the use of tissue biopsies. Furthermore, we used stable isotope tracers during the hyperinsulinemic-euglycemic clamps and during the assessment of brown adipose tissue activity in combination with PET scanning. We also used a metabolic chamber to assess sleeping metabolic rate upon NR supplementation and to control the indoor environment with a stable temperature during the cold acclimation study period. Collaborations within the ENERGISE consortium allowed us to use additional technologies, such as metabolomics, to obtain more results from the valuable muscle tissue samples. The combination of these advanced techniques can only be performed in a few research departments worldwide and leads to innovative translational research data.

Furthermore, many research has been performed on classic intervention strategies such as exercise training (endurance training, resistance training, high intensity interval training or a combination) and reducing caloric intake with various diets (such as low carbohydrate diet, high protein diet, low fat diet, low glycemic load diet, Mediterranean diet, paleo diet, intermittent fasting or a combination). These intervention strategies share a common goal: increasing energy expenditure and reducing energy intake resulting in a low cellular energy status. A low cellular energy status activates metabolic pathways that lead to metabolic adaptations which are favorable for human health, such as an increase in insulin sensitivity. The research presented in this thesis describes the investigation of novel approaches to improve insulin sensitivity by targeting the same metabolic pathways as are activated by exercise training and caloric restriction, but then with novel strategies: supplementation with the food supplement nicotinamide riboside, non-shivering cold acclimation, and replacing sitting time by low-intensity physical activities. 


\section{REFERENCES}

1. WHO. Factsheet obesity and overweight 2018 [Available from: https://www.who.int/newsroom/fact-sheets/detail/obesity-and-overweight.

2. WHO. Global report on diabetes. 2016.

3. RIVM. Kosten van ziekten 2017 [Available from: https://statline.rivm.nl/\#/RIVM/nl/dataset/50050NED/table?ts=1580224840032.

4. NASA. Scientific consensus: Earth's climate is warming 2009 [Available from: https://climate.nasa.gov/scientific-consensus/.

5. $\quad$ United Nations. Paris Agreement. 2016.

6. RIVM. Klimaatakkoord 2016 [Available from:

https://www.rijksoverheid.nl/onderwerpen/klimaatverandering/klimaatakkoord/wat-is-hetklimaatakkoord. 



\section{SUMMARY}

The worldwide prevalence of obesity and type 2 diabetes mellitus (T2DM) has dramatically increased over the past decades, and this development is also seen in the Dutch population. Obesity can lead to reduced insulin sensitivity and mitochondrial dysfunction, which are key characteristics of metabolic diseases such as T2DM. The fundamental cause of the high prevalence of obesity and T2DM is an unhealthy lifestyle characterized by a high caloric diet and insufficient physical activity. Exercise training and dietary caloric restriction are the two most commonly studied and most powerful lifestyle interventions to counteract obesity and T2DM. These intervention strategies share a common goal: inducing a low cellular energy status by increasing energy expenditure or reducing energy intake respectively. A low cellular energy status activates metabolic pathways that induce metabolic adaptations which are favorable for human health, such as an increase in insulin sensitivity and mitochondrial function. However, these strategies seem to be challenging to adhere to on the long term for people with obesity and T2DM. Therefore, it is important to investigate alternative strategies to prevent and treat obesity and T2DM. The research presented in this thesis describes the investigation of novel approaches to improve human insulin sensitivity and muscle mitochondrial function by targeting the same metabolic pathways as are activated by exercise training and caloric restriction, but with novel strategies. For this, three human intervention studies have been performed.

The first novel approach we investigated in this thesis was dietary supplementation with the $\mathrm{NAD}^{+}$-precursor nicotinamide riboside (NR). NAD+ is a critical signaling molecule of cellular energy status and is an essential substrate for the NAD+dependent enzyme sirtuin 1 . Sirtuin 1 is a metabolic regulator of oxidative energy metabolism and is activated in situations of a low cellular energy status. Since, NAD ${ }^{+}$ levels are decreased in the obese population, replenishment of $\mathrm{NAD}^{+}$levels was hypothesized to be a new strategy to improve metabolic health. Therefore, in chapter 2 and 3, the effect of six weeks $1000 \mathrm{mg} /$ day NR supplementation in healthy overweight and obese humans has been investigated on a wide range of metabolic health outcomes in a randomized placebo-controlled double-blinded cross-over intervention study. In chapter 2 we show that NR supplementation increased skeletal muscle $\mathrm{NAD}^{+}$metabolite levels NAAD and MeNAM, without affecting NAD ${ }^{+}$content itself. This suggests enhanced NAD+ turnover in skeletal muscle. Furthermore, NR induced changes in skeletal muscle acetylcarnitine metabolism, increased sleeping metabolic rate, and induced small improvements in body composition. However, 


\section{Appendices}

besides these modest effects, no other metabolic health effects were observed regarding insulin sensitivity, muscle mitochondrial function, ectopic lipid accumulation, whole body substrate oxidation, blood pressure, cardiac energy status and plasma markers of inflammation and energy metabolism. In chapter 3 , the effects of NR supplementation on brown adipose tissue (BAT) activity were evaluated. We show that NR supplementation in HFD-fed mice did enhance BAT activity. In addition, in vitro adipocytes derived from human BAT treated with NR did increase mitochondrial uncoupling. However, these promising preclinical results were not translated in humans, as the results from the human intervention study indicate that NR supplementation did not affect human BAT activity or cold induced thermogenesis.

The second novel approach we investigated in this thesis was non-shivering cold acclimation. In chapter 4 we investigated whether previously reported beneficial effects of ten days mild cold acclimation on peripheral insulin sensitivity were sustained for a longer period of time upon cessation of the cold exposure. Furthermore, we investigated whether these effects were accompanied by improvements in postprandial glucose and lipid metabolism and markers of cardiovascular health. For this, a human intervention study was performed in patients with T2DM who were exposed to mild cold $\left(16-17^{\circ} \mathrm{C}\right)$ for ten consecutive days. During the cold acclimation, we aimed to prevent shivering and targeted to investigate the effects of non-shivering thermogenesis. Using this approach, we could not reproduce the improvements in peripheral insulin sensitivity upon mild cold exposure that has previously been reported. Hence, also no improvements in the other outcomes were observed. To investigate the underlying reason for this discrepancy in results regarding insulin sensitivity, we performed additional analyses in skeletal muscle biopsies from the previous cold acclimation study and our current study. In samples from the previous cold acclimation study it was observed that the expression of genesets related to muscle contraction was induced along with increased GLUT4 translocation to the cell membrane. In the current study, specifically designed to prevent shivering, no such changes were observed. Jointly, this may indicate that shivering may have occurred in the previous study, which may have resulted in the induction of contraction related gene sets and more GLUT4 in the muscle cell membrane upon cold acclimation.

In recent years, both epidemiological and intervention studies have shown that replacement of sedentary time by light-intensity physical activities (such as standing and walking), as well as moderate-to-vigorous physical activities (such as exercise training) induced improvements in glucose homeostasis and markers of insulin 
sensitivity, suggesting improved metabolic health. Thus far, it is not known, however, if these improvements act via similar underlying pathways. Chapter 5 describes the third novel approach we investigated in this thesis. A randomized cross-over intervention study was performed in which healthy overweight and obese women underwent a four-day sit regime, exercise regime and sit less regime followed by metabolic assessments on the fifth day. We show that replacing sedentary time by several hours of standing and walking (sit less regime) was similarly effective in improving human peripheral insulin sensitivity compared to a single moderate-tovigorous exercise bout per day (exercise regime). This improvement in peripheral insulin sensitivity could not be explained by an increase in muscle mitochondrial function. Furthermore, hepatic insulin sensitivity and intrahepatic lipid accumulation were not affected by the four-day activity regimes.

Overall, the human intervention studies described in this thesis were designed to investigate novel approaches to improve human metabolic health with the main aim to improve human insulin sensitivity and muscle mitochondrial function. Taken together, NR supplementation and cold acclimation without shivering did not improve human insulin sensitivity or other important markers of metabolic health. It could be suggested that mild shivering upon cold exposure might be an effective strategy to increase peripheral insulin sensitivity. Furthermore, short term replacement of sitting time by standing and walking or by exercise is similarly effective in promoting human insulin sensitivity while these effects were not mediated by improved muscle mitochondrial function. 



\section{SAMENVATTING}

De prevalentie van obesitas en type 2 diabetes mellitus (T2DM) is wereldwijd sterk toegenomen in de afgelopen decennia, en deze ontwikkeling is ook zichtbaar in Nederland. Obesitas leidt tot een verminderde insuline gevoeligheid en mitochondriële functie, welke beide belangrijke kenmerken zijn van metabole ziekten zoals T2DM. Een ongezonde leefstijl bestaande uit een hoge calorie inname en weinig fysieke activiteit is een belangrijke oorzaak van de hoge prevalentie van obesitas en T2DM. De meest bestudeerde en effectieve leefstijlinterventies om obesitas en T2DM tegen te gaan, zijn sporten en een dieet laag in calorieën. Deze leefstijlinterventies zorgen beiden voor een negatieve energiebalans. Een negatieve energiebalans activeert specifieke metabole routes die ervoor zorgen dat onder andere insuline gevoeligheid en mitochondriële functie verbeteren. Dit is gunstig voor de metabole gezondheid. Echter, meer sporten en een lagere calorie inname zijn moeilijk vol te houden op de lange termijn voor mensen met obesitas en T2DM. Daarom is het belangrijk dat er onderzoek wordt gedaan naar alternatieve strategieën om obesitas en T2DM te behandelen en te voorkomen. Dit proefschrift beschrijft onderzoek naar nieuwe strategieën om insuline gevoeligheid en mitochondriële functie te verbeteren door middel van het activeren van dezelfde metabole routes die ook worden geactiveerd door sporten en een lagere calorie inname. Zodoende zijn er drie humane interventie studies uitgevoerd en beschreven in dit proefschrift.

De eerste nieuwe interventie strategie die is onderzocht in dit proefschrift is het voedingssupplement nicotinamide riboside (NR). NR wordt in het lichaam omgezet naar $\mathrm{NAD}^{+} . \mathrm{NAD}^{+}$is een molecuul dat in concentratie toeneemt bij een lage energie status in cellen, zoals bij een negatieve energiebalans. Daarnaast is $\mathrm{NAD}^{+}$een essentieel substraat voor het enzym sirtuin 1. Sirtuin 1 speelt een belangrijke rol in het regelen van het energie metabolisme. Concentraties van $\mathrm{NAD}^{+}$zijn verlaagd in mensen met obesitas. Er wordt dus verwacht dat een verhoging van $\mathrm{NAD}^{+}$ concentraties in deze doelgroep zal leiden tot een verbeterde metabole gezondheid. In hoofdstuk 2 en 3 is een studie beschreven waarin de effecten van zes weken 1000 mg/dag suppletie met NR in gezonde mensen met overgewicht en obesitas is onderzocht op verschillende metabole uitkomstmaten. De studie was gerandomiseerd, placebo-gecontroleerd, dubbelblind en had een cross-over onderzoeksopzet. In hoofdstuk 2 laten we zien dat suppletie met NR de concentratie van $\mathrm{NAD}^{+}$-gerelateerde metabolieten (NAAD en MeNAM) verhoogd in de spieren. De concentratie $\mathrm{NAD}^{+}$zelf veranderde niet. Dit suggereert dat er een verhoogde $\mathrm{NAD}^{+}$ turnover was in de spieren. Daarnaast zorgde NR-suppletie voor een verandering in 


\section{Appendices}

het acetylcarnitine metabolisme van de spier, een verhoogd energiegebruik tijdens de slaap en een kleine verbetering in de lichaamssamenstelling. Ondanks deze resultaten had NR-suppletie geen effect op de andere metabole uitkomstmaten: insuline gevoeligheid, spier mitochondriële functie, vetopslag in de spier en lever, substraat verbranding, bloeddruk, energie status van het hart en plasma markers voor inflammatie en het energie metabolisme. In hoofdstuk 3 worden de effecten van NRsuppletie op bruin vet activiteit beschreven. We laten zien dat in muizen NR-suppletie het bruin vet activeerde. Daarnaast laten we zien dat humane bruin vetcellen een verbeterde mitochondriële ontkoppeling hadden na toevoeging van NR in vitro. Desondanks zagen we deze resultaten niet terug in de humane interventie studie. Suppletie met NR in mensen met overgewicht en obesitas had namelijk geen effect op de bruin vet activiteit of op koude geïnduceerde thermogenese.

De tweede nieuwe interventie strategie die is onderzocht in dit proefschrift is koude acclimatisatie zonder rillen. In eerder onderzoek is aangetoond dat 10 dagen blootstelling aan milde kou de insuline gevoeligheid verbeterd. In hoofdstuk 4 hebben we onderzocht of deze verbetering in insuline gevoeligheid daarnaast ook langer aanwezig bleef na het stoppen van de blootstelling aan de kou. Daarnaast hebben we onderzocht of deze verbetering in insuline gevoeligheid gepaard ging met een verbetering in het postprandiale glucose- en vetmetabolisme en in een verbetering in markers voor de cardiovasculaire gezondheid. Om dit te onderzoeken hebben we een humane interventie studie uitgevoerd waarin patiënten met T2DM werden blootgesteld aan milde kou $\left(16-17^{\circ} \mathrm{C}\right)$ voor 10 opeenvolgende dagen. Omdat we het effect van koude acclimatisatie zonder rillen wilden onderzoeken, hebben we ervoor gezorgd dat de proefpersonen zo min mogelijk rilden tijdens de koude blootstelling. Tegen onze verwachting in, zagen we geen effect van de koude acclimatisatie op de insuline gevoeligheid op de korte termijn, zoals in een eerdere studie wel was aangetoond. Vervolgens zagen we dus ook geen effect van koude acclimatisatie op insuline gevoeligheid op de langere termijn en ook niet op de andere metabole uitkomstmaten. Om te onderzoeken waarom wij de effecten van koude acclimatisatie op insuline gevoeligheid niet konden reproduceren, hebben we extra analyses gedaan in de spierbiopten uit de vorige en de huidige studie. Uit de analyses van de spierbiopten uit de vorige studie bleek dat de expressie van genen die gerelateerd zijn aan spiercontracties verhoogd waren en tegelijk ook de translocatie van GLUT4 naar de celmembraan verhoogd was. In de analyses van de spierbiopten uit de huidige studie waren deze effecten niet zichtbaar. Deze resultaten duiden erop dat in de vorige studie wellicht enige vorm van rillen aanwezig was tijdens de koude blootstelling, en in huidige studie niet. Spiercontractie en GLUT4 translocatie in de 
vorige studie kan een verklaring zijn voor de gerapporteerde verbetering in insuline gevoeligheid.

De afgelopen jaren hebben epidemiologische en interventie studies aangetoond dat het vervangen van zittend gedrag door lichte fysieke activiteiten (zoals staan en wandelen), alsook door matig-tot-zware fysieke activiteiten (zoals sporten) het glucosemetabolisme kunnen verbeteren en waarschijnlijk ook insuline gevoeligheid en andere factoren gerelateerd aan de metabole gezondheid. Tot dusver is het nog onduidelijk of deze verbeteringen in het glucosemetabolisme bij de verschillende vormen van fysieke activiteit via dezelfde onderliggende metabole routes plaatsvinden. In hoofdstuk 5 zijn de resultaten beschreven van de derde nieuwe interventie strategie die is onderzocht in dit proefschrift. Een humane interventie studie is uitgevoerd waarbij gezonde vrouwen met overgewicht en obesitas in een gerandomiseerde volgorde drie verschillende activiteitenregimes hebben gevolgd: een zit regime, een sport regime en een minder-zitten regime. Het sport regime bestond uit 1 uur sporten per dag met een matig-tot-zware intensiteit. Het minderzitten regime bestond uit 4 uur staan en 3 uur wandelen per dag. De rest van tijd in het zit, sport en minder-zitten regime moest voornamelijk zittend worden doorgebracht. Elk activiteitenregime duurde 4 dagen en op de $5^{\mathrm{e}}$ dag werden verschillende metingen verricht. We laten zien dat het vervangen van zittend gedrag door staan en wandelen (minder-zitten regime) net zo effectief was in het verbeteren van de spier insuline gevoeligheid als 1 uur sporten per dag (sport regime). Deze verbetering van de spier insuline gevoeligheid kon niet worden verklaard door een toename in spier mitochondriële functie. Verder lieten de resultaten zien dat de lever insuline gevoeligheid en de vetopslag in de lever niet veranderden na een activiteitenregime van 4 dagen.

Samenvattend, in dit proefschrift zijn drie humane interventie studies beschreven die waren ontworpen om nieuwe strategieën te onderzoeken om de humane metabole gezondheid te verbeteren met als hoofddoel het verbeteren van insuline gevoeligheid en spier mitochondriële functie. Er kan geconcludeerd worden dat NR-suppletie en koude acclimatisatie zonder rillen de insuline gevoeligheid en andere belangrijke parameters van metabole gezondheid niet kunnen verbeteren. Het wordt gesuggereerd dat een milde vorm van rillen tijdens koude acclimatisatie een manier is om insuline gevoeligheid te verbeteren. Tenslotte, 4 dagen vervanging van zittend gedrag door staan en wandelen is net zo effectief in het verbeteren van de spier insuline gevoeligheid als 1 uur sporten per dag en deze verbetering kan niet worden verklaard door een verbeterde spier mitochondriële functie. 



\section{DANKWOORD}

Dit proefschrift was nooit tot stand gekomen zonder de hulp van velen. Ik wil dan ook graag iedereen bedanken die op wat voor manier dan ook heeft bijgedragen.

Allereerst wil ik mijn promotieteam bedanken. Bedankt dat jullie mij de kans hebben gegeven om deze promotie te doen. Op wetenschappelijk en persoonlijk vlak heb ik veel geleerd in deze periode. Patrick, ik waardeer je kritische blik, heldere uitleg en efficiënte aanpak. Esther, zonder jouw vertrouwen en hulp had hier nu geen boekje gelegen. Bedankt dat je altijd (echt altijd) voor me klaarstond en samen met mij naar een oplossing zocht. Matthijs, jij bent wat later aangesloten bij ons team en jouw frisse blik en final touch waren erg waardevol.

Daarnaast wil ik ook nog een paar anderen bedanken die officieel geen deel uitmaken van het promotieteam, maar wel een belangrijke bijdrage hebben geleverd. Vera, bedankt voor je hulp bij het uitwerken en interpreteren van de MRS data. Joris, dankjewel voor het delen van je oxygraaf kennis en het controleren van de data. Wouter, bedankt voor de samenwerking in de koude acclimatisatie studie. Bas, fijn dat je de medisch verantwoordelijk arts wilden zijn in mijn studies en dank voor de hulp bij medisch gerelateerde zaken.

I would like to thank Prof. dr. Ronald Mensink, Dr. Denis Blondin, Prof. dr. Martijn Brouwers, Prof. dr. Luc van Loon and Dr. Hidde van der Ploeg, the members of the reading committee, for their time and effort in reviewing my thesis.

Kay en Anne, wat ben ik blij dat jullie mijn paranimfen willen zijn. Anne, ondanks dat we collega's zijn, is onze vriendschap niet echt op het werk ontstaan, maar vooral daarbuiten. Door onze gezamenlijke passie voor het hardlopen hebben we heel wat uren (met zijn tweetjes, met de SHOCk runningclub of bij de hardloopvereniging JustGoo) samen rennend doorgebracht. Dankjewel voor je luisterende oor en de gezellige gesprekken over van alles en nog wat. Ook aan de reisjes naar de Ardennen, Portugal, Oostenrijk en IJsland heb ik mooie herinneringen overgehouden. Kay, vanaf het begin van onze PhD hebben wij de testdagen samen uitgevoerd. Ik kon me geen fijnere clamp-buddy wensen. Je behulpzaamheid, geduld, vrolijkheid en positieve houding hebben zeker bijgedragen aan de fijne samenwerking. Naast die ontelbare clamps hebben we ook tijdens de pauzes en buiten werktijd om veel tijd samen doorgebracht. Samen met de andere Limburgers "achter in de gang", Vera, Bas en Yvo, hebben we een hoop lol gehad. Bedankt voor jullie afleiding, 


\section{Appendices}

gekkigheid, humor en heerlijke zelfgebakken vlaai en cake. Vera, een speciaal woord van dank voor jou. Je betrokkenheid, aanstekelijke enthousiasme en onvermoeibare nieuwsgierigheid droegen zeker bij aan de plezierige werksfeer.

Marie-Fleur, samen met jou ben ik aan het CVON-Energise project begonnen en hebben we de koude acclimatisatie studie gestart. Bedankt voor de fijne samenwerking. Michiel, na een jaar heb jij het stokje van Marie-Fleur overgenomen. Naast de koude acclimatisatie studie hebben we ook in de NR studie veel samengewerkt. Enorm bedankt voor al je hulp.

Natuurlijk wil ik ook graag alle andere collega's binnen SHOCk (DMRG) bedanken. Het uitvoeren van een onderzoek is teamwork en doe je niet alleen. Speciale dank voor Johanna, Gert en Esther K, voor het invriezen van spierbiopten, uitvoeren van analyses en oxygraaf metingen. Daarnaast wil ik Niels, Vera en Michiel bedanken voor het nemen van de spierbiopten. Kim, samen hebben we een hoop uren doorgebracht achter de MRI-scanner in de vroege ochtend- en de late avonduurtjes, dankjewel voor al je hulp. Lena, Stephanie, Bernard en Maaike, bedankt voor de samenwerking binnen de SITLESS4 studie. Daarnaast wil ik ook graag mijn stagiaires Lonne, Anna en Michelle bedanken. Zonder jullie helpende hand was het mij niet gelukt om de verschillende studies te combineren. Uiteraard ook een woord van dank aan alle proefpersonen die hebben deelgenomen aan de studies. Zonder jullie was er geen onderzoek.

Lea, Mathijs, Jakob en Edmundo, wat ben ik blij dat jullie mijn kamergenootjes waren. Naast de drukte van alle studies, was het fijn om even op adem te kunnen komen in ons rustige kantoor. Thanks, merci, gracias en danke schön voor de prettige sfeer en gezellige gesprekken.

In het bijzonder wil ik ook mijn vrienden en (schoon)familie bedanken voor hun steun. Mama, dat ik zo ver ben gekomen, heb ik voor een groot deel aan jou te danken. Je hebt me altijd gesteund in mijn keuzes die me tot hier hebben gebracht. Ook wil ik jou, Sander, bedanken dat je altijd interesse toont in alles wat ik doe.

Lieve Jeroen, wat vind ik het fijn om met jou samen te zijn. Bedankt voor al je steun en liefde. Je relativeringsvermogen heeft me vaak geholpen om mijn PhD in perspectief te plaatsen in tijden dat ik erin verzoop. Ook hebben we een aantal mooie reizen gemaakt, waardoor er even tijd was om helemaal af te schakelen van het PhDleven. Ik hoop dat we samen nog veel avonturen zullen beleven in de toekomst. Ik hou van jou! 




\section{LIST OF PUBLICATIONS}

Remie CME, Roumans KHM, Moonen MPB, Connell NJ, Havekes B, Mevenkamp J, et al. Nicotinamide riboside supplementation alters body composition and skeletal muscle acetylcarnitine concentrations in healthy obese humans. Am J Clin Nutr. 2020 Apr 22; nqaa072. Online ahead of print.

Roumans KHM, Lindeboom L, Veeraiah P, Remie CME, Phielix E, Havekes B, et al. Hepatic saturated fatty acid fraction is associated with de novo lipogenesis and hepatic insulin resistance. Nat Commun. 2020 Apr 20;11(1):1891.

Remie CME*, Moonen MPB*, Roumans KHM, Havekes B, Gemmink A, Nascimento EBM, et al. Non-shivering cold acclimation does not improve postprandial glucose and lipid metabolism or insulin sensitivity in patients with type 2 diabetes. In preparation.

* These authors contributed equally

Remie CME, Bilet L, Duvivier BMFM, Bergman M, Roumans KHM, Huysmans SMD, et al. Replacing sedentary time with standing and walking is similar effective in enhancing insulin sensitivity as moderate-to-vigorous exercise. In preparation.

Nascimento EBM ${ }^{\star}$, Moonen MPB*, Remie CME, Gariani K, Jörgensen JA, Schaart G, et al. Increasing NAD+ levels through nicotinamide riboside activates in vivo brown adipose tissue in mice, but not in humans. Submitted.

* These authors contributed equally 



\section{ABOUT THE AUTHOR}

Carlijn Remie was born on March 12 $2^{\text {th }}, 1991$ in Sittard, the Netherlands. After finishing secondary education at Trevianum Sittard in 2009, she started the bachelor Nutrition and Dietetics at the HAN University of Applied Sciences in Nijmegen. After successfully completing her Bachelor's degree in 2013, Carlijn continued her education with the Master Nutrition and Health at the Wageningen University. She performed her Master thesis at the Department of Human and Animal Physiology of Wageningen University investigating the effects of carnitine supplementation on acute response to hypoxia in humans. For her Master internship she visited the University of Copenhagen, department of Biomedical Sciences, for five months. During her internship in Copenhagen, she investigated the effects of intermittent fasting on intramyocellular triglyceride content of human skeletal muscle and assisted in conducting a human clinical trial. In 2015, Carlijn received her Master's degree and subsequently started her PhD at the department of Nutrition and Movement Sciences at Maastricht University under the supervision of Prof. dr. Patrick Schrauwen, Dr. Esther Phielix and Prof. dr. Matthijs Hesselink. The research conducted during this period, as described in this PhD thesis, was primarily focused on the investigation of novel approaches to improve human insulin sensitivity and muscle mitochondrial function. 